\title{
Synthesis and Application of Chiral Spiro Cp Ligands in Rhodium-Catalyzed Asymmetric Oxidative Coupling of Biaryl Compounds with Alkenes
}

\author{
Jun Zheng, Wen-Jun Cui, Chao Zheng and Shu-Li You*
}

State Key Laboratory of Organometallic Chemistry

Shanghai Institute of Organic Chemistry, Chinese Academy of Sciences

345 Lingling Lu, Shanghai 200032, China

Collaborative Innovation Center of Chemical Science and Engineering, Tianjin, China

E-mail: $\underline{\text { slyou@ sioc.ac.cn }}$

Table of Contents

General methods

S2

Synthesis of ligands

S3-S12

Synthesis of Rh complexes

S13-S15

General procedure for asymmetric oxidative coupling reactions

S16-S27

X-Ray crystal structures of $(\boldsymbol{S})$-K3a and $(\boldsymbol{S})$-K3d

$\mathrm{S} 28-\mathrm{S} 30$

Copies of NMR spectra and HPLC chromatographs

S31-S119 
General Methods. Unless stated otherwise, all reactions were carried out in flame-dried glassware under a dry argon atmosphere. All solvents were purified and dried according to standard methods prior to use.

${ }^{1} \mathrm{H}$ and ${ }^{13} \mathrm{C}$ NMR spectra were recorded on a Varian instrument $(400 \mathrm{MHz}$ and 100 $\mathrm{MHz}$, respectively) or an Agilent instrument $(400,600 \mathrm{MHz}$ and 100, $151 \mathrm{MHz}$, respectively) and internally referenced to tetramethylsilane signal or residual protio solvent signals. Data for ${ }^{1} \mathrm{H}$ NMR are recorded as follows: chemical shift ( $\left.\delta, \mathrm{ppm}\right)$, multiplicity $(\mathrm{s}=$ singlet, $\mathrm{d}=$ doublet, $\mathrm{t}=$ triplet, $\mathrm{sept}=$ septet, $\mathrm{m}=$ multiplet or unresolved, br = broad singlet, coupling constant (s) in $\mathrm{Hz}$, integration). Data for ${ }^{13} \mathrm{C}$ NMR and ${ }^{19} \mathrm{~F}$ NMR are reported in terms of chemical shift $(\delta, \mathrm{ppm})$.

(S)-1,1'-Spirobiindanyl-7,7'-dicarboxylic acid $(S)$-1 and (S)-7,7'-dichloromethyl-1,1'spirobiindane $(S)-\mathbf{5 d}$ were prepared according to published procedures. ${ }^{1}$ 


\section{Synthesis of ligands:}

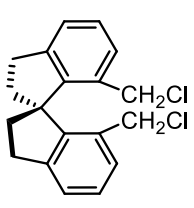

(S)-5d

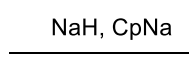

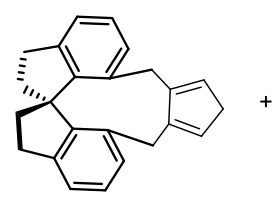

(S)-6d : 20\% yield

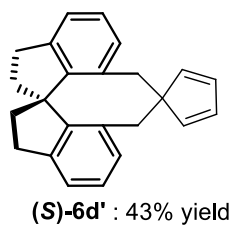

(S)-6d' : $43 \%$ yield

To a cold $\left(-20^{\circ} \mathrm{C}\right)$ suspension of sodium hydride $(11.2 \mathrm{mg}, 0.28 \mathrm{mmol}, 1.40$ equiv.) in THF $(1.0 \mathrm{~mL})$ was added dropwise a solution of sodium cyclopentadienylide $(0.12$ $\mathrm{mL}, 0.24 \mathrm{mmol}, 1.0 \mathrm{M}$ in THF, 1.20 equiv.) followed by a solution of $(S)-5 \mathbf{d}(61.3 \mathrm{mg}$, $0.2 \mathrm{mmol})$ in THF $(2.0 \mathrm{~mL})$. The mixture was warmed up slowly and stirred for 24 hours, then quenched carefully with water $(5.0 \mathrm{~mL})$ and extracted with diethyl ether (2 X $5.0 \mathrm{~mL}$ ). The combined organic layers were dried over $\mathrm{Na}_{2} \mathrm{SO}_{4}$, filtered and concentrated under reduced pressure. The residue was then purified by silica gel column chromatography (petroleum $\rightarrow$ petroleum:EtOAc=100:1), affording $26.6 \mathrm{mg}$ (43\% yield) of (S)-6d' and $12.3 \mathrm{mg}$ (20\% yield) of $(S)$-6d.

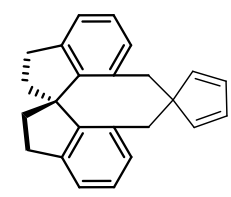

(S) $-6 d^{\prime}$

White solid, m.p. $=180-181{ }^{\circ} \mathrm{C}, 26.6 \mathrm{mg}, 43 \%$ yield. ${ }^{1} \mathrm{H}$ NMR $\left(400 \mathrm{MHz}, \mathrm{CDCl}_{3}\right) \delta$ $7.15(\mathrm{~s}, 4 \mathrm{H}), 6.85(\mathrm{~s}, 2 \mathrm{H}), 6.27(\mathrm{~s}, 2 \mathrm{H}), 6.06(\mathrm{~s}, 2 \mathrm{H}), 3.13(\mathrm{~d}, J=12.8 \mathrm{~Hz}, 2 \mathrm{H})$, 3.12-2.96 (m, 2H), 2.88-2.82 (m, 2H), 2.29-2.24 (m, 2H), 1.98-1.90 (m, 2H), 1.79 (d, $J=12.4 \mathrm{~Hz}, 2 \mathrm{H}) ;{ }^{13} \mathrm{C} \mathrm{NMR}\left(100 \mathrm{MHz}, \mathrm{CDCl}_{3}\right) \delta 149.0,143.6,142.8,135.0,128.9$, 128.7, 126.9, 123.0, 61.4, 60.5, 38.2, 34.6, 30.5; IR (thin film): $v_{\max }\left(\mathrm{cm}^{-1}\right)=3060$, 2924, 2853, 1703, 1592, 1447, 756; HRMS (EI) calcd for $\mathrm{C}_{24} \mathrm{H}_{22}[\mathrm{M}]^{+}:$: 310.1722; Found: $310.1724 ;[\alpha]_{\mathrm{D}}^{28}=-321.1\left(\mathrm{c}=0.20, \mathrm{CHCl}_{3}\right)$.

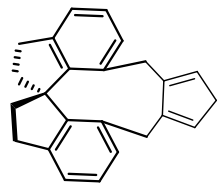

(s)-6d

White solid, m.p. $=142-143{ }^{\circ} \mathrm{C}, 12.3 \mathrm{mg}, 20 \%$ yield. ${ }^{1} \mathrm{H}$ NMR $\left(400 \mathrm{MHz}, \mathrm{CDCl}_{3}\right) \delta$ 7.12-7.09 (m, 4H), 7.01-6.87 (m, 2H), $6.03(\mathrm{~s}, 2 \mathrm{H}), 3.47\left(\mathrm{AB}, J_{A B}=13.2 \mathrm{~Hz}, 2 \mathrm{H}\right), 3.36$ $\left(\mathrm{BA}, J_{B A}=13.6 \mathrm{~Hz}, 2 \mathrm{H}\right), 3.07-2.99(\mathrm{~m}, 2 \mathrm{H}), 2.89(\mathrm{dd}, J=16.0,8.4 \mathrm{~Hz}, 1 \mathrm{H}), 2.76(\mathrm{~s}$, 
2H), $2.32(\mathrm{dd}, J=12.4,6.8 \mathrm{~Hz}, 1 \mathrm{H}), 2.05-1.94(\mathrm{~m}, 2 \mathrm{H}) ;{ }^{13} \mathrm{C} \mathrm{NMR}\left(100 \mathrm{MHz}, \mathrm{CDCl}_{3}\right)$ $\delta 147.4,146.4,143.1,135.4,129.0,127.7,127.3,122.7,61.2,38.4,38.3,31.8,30.4$; IR (thin film): $v_{\max }\left(\mathrm{cm}^{-1}\right)=3069,2925,2853,1928,1592,1447,1370,761$; HRMS (EI) calcd for $\mathrm{C}_{24} \mathrm{H}_{22}[\mathrm{M}]^{+}$: 310.1722 ; Found: 310.1718; $[\alpha]_{\mathrm{D}}{ }^{28}=+352.6(\mathrm{c}=0.20$, $\left.\mathrm{CHCl}_{3}\right)$.

The $(S)-\mathbf{6 d}$ ' (79.5 mg, $0.21 \mathrm{mmol})$ and $\mathrm{K}_{2} \mathrm{CO}_{3}(2.9 \mathrm{mg}, 0.21 \mathrm{mmol})$ was added into degassed dodecane $(21.0 \mathrm{~mL})$ and stirred at $240^{\circ} \mathrm{C}$ for 16 hours in a sealed tube. After cooling down to rt, the solvent was evaporated under reduced pressure and the residue was purified by column chromatography on silica gel (ethyl acetate/petroleum ether $=$ 1:100) affording $79.0 \mathrm{mg}$ (99\% yield) of $(S)-\mathbf{6 b}$.

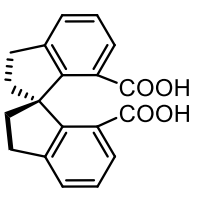

$(S)-1$

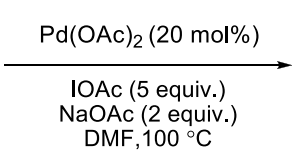
DMF, $100^{\circ} \mathrm{C}$

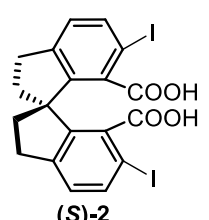

(S)-2

The diiodination compound $(S)-\mathbf{2}$ was prepared according to literature procedures ${ }^{2}$, with the following modifications. Substrate $(S)-\mathbf{1}(0.92 \mathrm{~g}, 3 \mathrm{mmol}), \operatorname{Pd}(\mathrm{OAc})_{2}(134.7$ $\mathrm{mg}, 0.6 \mathrm{mmol})$, iodobenzene diacetate $(2.42 \mathrm{~g}, 7.5 \mathrm{mmol})$ and $\mathrm{I}_{2}(1.9 \mathrm{~g}, 7.5 \mathrm{mmol})$ were dissolved in DMF (30 mL) under argon. The tube was sealed with a cap and the reaction mixture was stirred at $100{ }^{\circ} \mathrm{C}$ for 36 hours. The reaction mixture was cooled to room temperature. After the solvent was evaporated to vacuum, saturated $\mathrm{Na}_{2} \mathrm{SO}_{3}$ $(30 \mathrm{~mL})$ was added. The mixture was acidified with $2 \mathrm{~N} \mathrm{HCl}(30 \mathrm{~mL})$, extracted with DCM $(30 \mathrm{~mL} \times 5)$ and dried over $\mathrm{Na}_{2} \mathrm{SO}_{4}$. The solvent was removed in a rotary evaporator and the residue was purified by column chromatography on silica gel (petroleum: ethyl acetate $=4: 1 \rightarrow$ petroleum: ethyl acetate: acetate acid $=4: 1: 0.01$ ) affording $1.17 \mathrm{~g}$ (70\% yield) of the diiodination product $(S)$-2.

White solid, m.p. $>300{ }^{\circ} \mathrm{C}$. ${ }^{1} \mathrm{H}$ NMR (400 MHz, DMSO- $\left.d_{6}\right) \delta 8.19$ (d, $J=8.0 \mathrm{~Hz}$, $2 \mathrm{H}), 7.56(\mathrm{~d}, J=8.0 \mathrm{~Hz}, 2 \mathrm{H}), 3.48-3.35(\mathrm{~m}, 4 \mathrm{H}), 2.95-2.87(\mathrm{~m}, 2 \mathrm{H}), 2.80-2.69(\mathrm{~m}$, $2 \mathrm{H}) ;{ }^{13} \mathrm{C}$ NMR (100 MHz, DMSO- $\left.d_{6}\right) \delta 169.4,146.3,145.1,138.1,138.0,127.2,91.5$, 62.7, 39.4, 30.1; IR (thin film): $v_{\max }\left(\mathrm{cm}^{-1}\right)=2956,2924,2853,1749,1663,1450$, 
1328, 1261, 808; HRMS (ESI) calcd for $\mathrm{C}_{19} \mathrm{H}_{18} \mathrm{I}_{2} \mathrm{NO}_{4}\left[\mathrm{M}+\mathrm{NH}_{4}\right]^{+}$: 577.932; Found: $577.9319 ;[\alpha]_{\mathrm{D}}^{28}=-15.2(\mathrm{c}=0.10, \mathrm{MeOH})$.

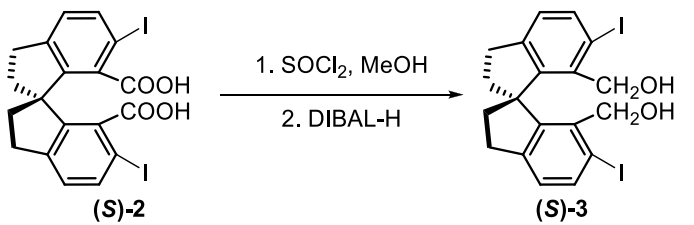

To diiodination compound $(S)-2(100.0 \mathrm{mg}, 0.178 \mathrm{mmol})$ was added $\mathrm{SOCl}_{2}(2 \mathrm{~mL})$ in a dry two-neck flask with a stirring bar under argon atmosphere. The mixture was refluxed for $8 \mathrm{~h}$ and excess $\mathrm{SOCl}_{2}$ was removed under reduced pressure. Then, $\mathrm{MeOH}$ $(1 \mathrm{~mL})$ was added and the mixture was heated to reflux for $4 \mathrm{~h}$. The reaction mixture was cooled to room temperature, and the residue was purified by column chromatography on silica gel (petroleum: ethyl acetate $=40: 1)$ affording $99.5 \mathrm{mg}(95 \%$ yield) of esterification product.

White solid, m.p. $=143-144{ }^{\circ} \mathrm{C} .{ }^{1} \mathrm{H}$ NMR $\left(400 \mathrm{MHz} \mathrm{CDCl}_{3}\right) \delta 7.66(\mathrm{~d}, J=8.0 \mathrm{~Hz}$, 2H), $7.00(\mathrm{~d}, J=8.0 \mathrm{~Hz}, 2 \mathrm{H}), 3.24(\mathrm{~s}, 6 \mathrm{H}), 3.05-2.87(\mathrm{~m}, 4 \mathrm{H}), 2.64-2.56(\mathrm{~m}, 2 \mathrm{H})$, 2.24-2.18 (m, 2H); ${ }^{13} \mathrm{C}$ NMR $\left(100 \mathrm{MHz}, \mathrm{CDCl}_{3}\right) \delta 167.7,147.2,145.2,137.9,136.2$, 127.1, 90.3, 62.6, 51.3, 39.7, 30.2; IR (thin film): $v_{\max }\left(\mathrm{cm}^{-1}\right)=2942,2923,2872,1721$, 1567, 1429, 1279, 1250, 798; HRMS (ESI) calcd for $\mathrm{C}_{21} \mathrm{H}_{22} \mathrm{I}_{2} \mathrm{NO}_{4}\left[\mathrm{M}+\mathrm{NH}_{4}\right]^{+}$: 605.9633; Found: 605.9633; $[\alpha]_{\mathrm{D}}{ }^{26}=-299.2\left(\mathrm{c}=0.20, \mathrm{CHCl}_{3}\right)$.

DIBAL-H (1.5 M in PhMe, $0.68 \mathrm{~mL}, 1.02 \mathrm{mmol}$ ) was added dropwise to a stirred and cooled $\left(-78{ }^{\circ} \mathrm{C}\right)$ solution of above esterification product $(99.5 \mathrm{mg}, 0.17 \mathrm{mmol})$ in PhMe $(2 \mathrm{~mL})$. After stirring at $-78{ }^{\circ} \mathrm{C}$ for $3 \mathrm{~h}$, the cooling bath was removed. The reaction mixture was stirred at $\mathrm{rt}$ for $5 \mathrm{~h}$. The mixture was quenched with saturated aqueous $\mathrm{NH}_{4} \mathrm{Cl}(5 \mathrm{~mL})$ and extracted with ethyl acetate $(5 \mathrm{~mL} \times 3)$. The combined organic extracts were dried over $\mathrm{Na}_{2} \mathrm{SO}_{4}$ and evaporated under vacuum. The residue was purified by column chromatography on silica gel (petroleum: ethyl acetate $=5: 1$ ) affording $83.9 \mathrm{mg}$ (93\% yield) of (S)-3.

White solid, m.p. $=164-165{ }^{\circ} \mathrm{C} .{ }^{1} \mathrm{H}$ NMR $\left(400 \mathrm{MHz}, \mathrm{CDCl}_{3}\right) \delta 7.74(\mathrm{~d}, J=8.0 \mathrm{~Hz}$, $2 \mathrm{H}), 6.94(\mathrm{~d}, J=8.0 \mathrm{~Hz}, 2 \mathrm{H}), 4.39\left(\mathrm{AB}, J_{A B}=12.0 \mathrm{~Hz}, 2 \mathrm{H}\right), 4.20\left(\mathrm{BA}, J_{B A}=12.0 \mathrm{~Hz}\right.$, $2 \mathrm{H}), 3.37(, 2 \mathrm{H}), 3.06-2.82(\mathrm{~m}, 4 \mathrm{H}), 2.31-2.26(\mathrm{~m}, 2 \mathrm{H}), 2.04-1.95(\mathrm{~m}, 2 \mathrm{H}) ;{ }^{13} \mathrm{C} \mathrm{NMR}$ 
$\left(100 \mathrm{MHz}, \mathrm{CDCl}_{3}\right) \delta 150.1,143.9,139.2,137.9,126.5,100.4,63.0,40.0,30.1$; IR (thin film): $v_{\max }\left(\mathrm{cm}^{-1}\right)=3226,2951,2935,2878,2843,1406,1003$, 809; Anal. calcd for $\mathrm{C}_{19} \mathrm{H}_{18} \mathrm{I}_{2} \mathrm{O}_{2}$ : C, 42.88; H, 3.41; Found: C, 42.92; H, 3.47; $[\alpha]_{\mathrm{D}}{ }^{27}=-239.0(\mathrm{c}=0.20$, $\left.\mathrm{CHCl}_{3}\right)$.

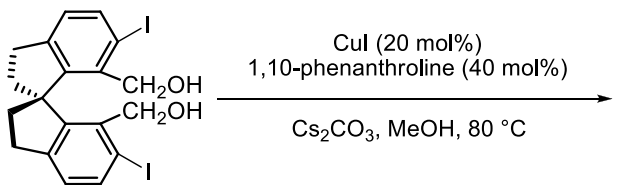

$(S)-3$

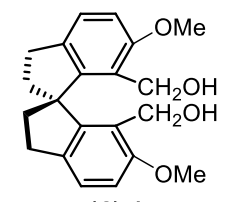

$(S)-4 a$

The copper-catalyzed coupling of aryl iodides with aliphatic alcohols was prepared according to literature procedures. ${ }^{3} \mathrm{~A}$ test tube was charged with $\mathrm{CuI}(38.1 \mathrm{mg}, 0.2$ mmol, 0.20 equiv), 1, 10-phenanthroline ( $72.1 \mathrm{mg}, 0.4 \mathrm{mmol}, 0.40$ equiv), $\mathrm{Cs}_{2} \mathrm{CO}_{3}$ (912.2 mg, $2.8 \mathrm{mmol}, 2.8$ equiv), (S)-3 (532.2 mg, $1.0 \mathrm{mmol}, 1.0$ equiv) and methanol $(5 \mathrm{~mL})$. The test tube was sealed and the reaction mixture was stirred at $80{ }^{\circ} \mathrm{C}$ for $37 \mathrm{~h}$. The resulting suspension was cooled to room temperature and filtered through a $0.5 \times$ $1 \mathrm{~cm}$ pad of silica gel, eluting with diethyl ether. The filtrate was concentrated under vacuum. Purification of the residue by flash chromatography on silica gel (petroleum: ethyl acetate $=1: 1)$ provided $276.0 \mathrm{mg}(81 \%$ yield $)$ of $(S)-\mathbf{4 a}$.

White solid, m.p. $=202-204{ }^{\circ} \mathrm{C} .{ }^{1} \mathrm{H}$ NMR $\left(400 \mathrm{MHz}, \mathrm{CDCl}_{3}\right) \delta 7.16(\mathrm{~d}, J=8.4 \mathrm{~Hz}$, $2 \mathrm{H}), 6.80(\mathrm{~d}, J=8.4 \mathrm{~Hz}, 2 \mathrm{H}), 4.39\left(\mathrm{AB}, J_{A B}=12.0 \mathrm{~Hz}, 2 \mathrm{H}\right), 4.29\left(\mathrm{BA}, J_{B A}=12.0 \mathrm{~Hz}\right.$, 2H), $3.86(\mathrm{~s}, 6 \mathrm{H}), 3.48(\mathrm{~s}, 2 \mathrm{H}), 2.92-2.91(\mathrm{~m}, 4 \mathrm{H}), 2.30-2.28(\mathrm{~m}, 2 \mathrm{H}), 2.00-1.92(\mathrm{~m}$, $2 \mathrm{H}) ;{ }^{13} \mathrm{C} \mathrm{NMR}\left(100 \mathrm{MHz}, \mathrm{CDCl}_{3}\right) \delta 158.5,149.2,135.2,124.5,109.7,61.7,56.6$, 55.8, 40.2, 29.6; IR (thin film): $v_{\max }\left(\mathrm{cm}^{-1}\right)=3483,3384,2928,1592,1476,1461$, 1253, 978, 787; Anal. calcd for $\mathrm{C}_{21} \mathrm{H}_{24} \mathrm{O}_{4}$ : C, 74.09; H, 7.11; Found: C, 73.91; H, 7.27; $[\alpha]_{\mathrm{D}}^{26}=-89.8\left(\mathrm{c}=0.20, \mathrm{CHCl}_{3}\right)$.

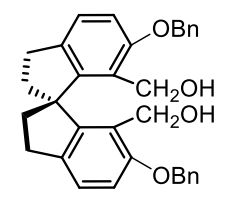

(S) $-4 b$

(S)-4b was prepared by following the same procedure as the synthesis of $(S)-\mathbf{4 a}$, except that benzyl alcohol was used instead of methanol. (S)-4b: Colorless oil, 379.4 $\mathrm{mg}, 64 \%$ yield (1.2 mmol scale). ${ }^{1} \mathrm{H}$ NMR (400 MHz, $\left.\mathrm{CDCl}_{3}\right) \delta$ 7.42-7.40 (m, 4H), 
7.37-7.33 (m, 4H), 7.30-7.27 (m, 2H), $7.12(\mathrm{~d}, J=8.4 \mathrm{~Hz}, 2 \mathrm{H}), 6.84(\mathrm{~d}, J=8.4 \mathrm{~Hz}$, $2 \mathrm{H}), 5.14\left(\mathrm{AB}, J_{A B}=12.0 \mathrm{~Hz}, 2 \mathrm{H}\right), 5.07\left(\mathrm{BA}, J_{B A}=12.0 \mathrm{~Hz}, 2 \mathrm{H}\right), 4.42\left(\mathrm{AB}, J_{A B}=\right.$ $12.0 \mathrm{~Hz}, 2 \mathrm{H}), 4.31\left(\mathrm{BA}, J_{B A}=12.0 \mathrm{~Hz}, 2 \mathrm{H}\right), 3.38$ (brs, 2H), 2.93-2.89 (m, 4H), 2.33-2.27 (m, 2H), 2.04-1.95 (m, 2H); $\left.{ }^{13} \mathrm{C} \mathrm{NMR} \mathrm{(100} \mathrm{MHz,} \mathrm{CDCl}_{3}\right) \delta$ 157.6, 149.4, 137.0, 135.7, 128.5, 127.8, 127.2, 125.1, 124.5, 111.4, 70.5, 61.7, 56.8, 40.2, 29.7; IR (thin film): $v_{\max }\left(\mathrm{cm}^{-1}\right)=3564,3394,2941,1592,1453,748$; HRMS (EI) calcd for $\mathrm{C}_{33} \mathrm{H}_{32} \mathrm{NaO}_{4}[\mathrm{M}+\mathrm{Na}]^{+}:$515.2193; Found: 515.2192; $[\alpha]_{\mathrm{D}}{ }^{29}=-146.2\left(\mathrm{c}=0.15, \mathrm{CHCl}_{3}\right)$.

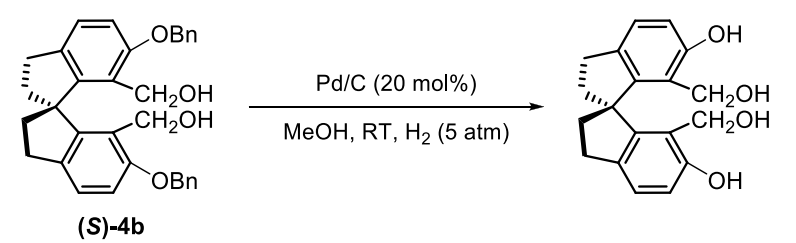

(S)-4b (246.0 mg, $0.5 \mathrm{mmol}, 1.0$ equiv) was dissolved in methanol (3 mL). Pd on charcoal (10 percent, $100 \mathrm{mg}, 0.2$ equiv) was added, and the flask was purged three times with hydrogen. The reaction mixture was stirring for $24 \mathrm{~h}$ under $5 \mathrm{~atm}$ of hydrogen. Then, Pd catalyst was removed by filtration through Celite. The filtrate was concentrated and absorbed on silica gel. Purification by flash chromatography on silica gel (petroleum: ethyl acetate $=5: 1)$ provided $75.9 \mathrm{mg}(49 \%$ yield $)$ of the diphenol product.

White solid, m.p. $=150-151{ }^{\circ} \mathrm{C}, 75.9 \mathrm{mg}, 49 \%$ yield. ${ }^{1} \mathrm{H}$ NMR $\left(400 \mathrm{MHz}, \mathrm{CDCl}_{3}\right) \delta$ 7.26 (brs, 2H), 7.07 (d, $J=8.0 \mathrm{~Hz}, 2 \mathrm{H}), 6.70$ (d, $J=8.0 \mathrm{~Hz}, 2 \mathrm{H}), 4.43-4.29$ (m, 6H), 3.12-2.73 (m, 4H), 2.33-2.28 (m, 2H), 1.85-1.77 (m, 2H); ${ }^{13} \mathrm{C}$ NMR (100 MHz, $\left.\mathrm{CDCl}_{3}\right) \delta 155.9,148.0,135.1,125.3,121.7,115.2,61.3,57.2,39.9,29.8$; IR (thin film): $v_{\max }\left(\mathrm{cm}^{-1}\right)=3454,3313,2924,2850,1595,1476,1450,1261,1006,811$; HRMS (EI) calcd for $\mathrm{C}_{19} \mathrm{H}_{20} \mathrm{O}_{4}[\mathrm{M}]^{+}: 312.1361$; Found: $312.1359 ;[\alpha]_{\mathrm{D}}{ }^{29}=-123.7(\mathrm{c}$ $\left.=0.20, \mathrm{CHCl}_{3}\right)$.
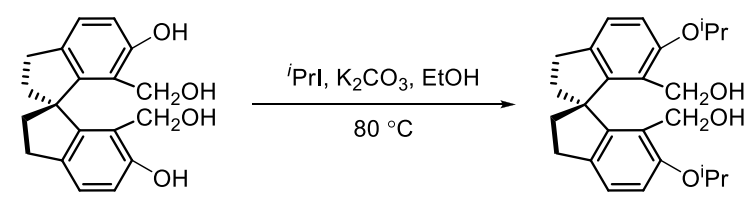

(S)-4c

A mixture of the diphenol (113 mg, $0.36 \mathrm{mmol}, 1$ equiv.), 2-isopropyl iodide (0.15 $\mathrm{mL}, 4$ equiv.) and $\mathrm{K}_{2} \mathrm{CO}_{3}(299.7 \mathrm{mg}, 6$ equiv.) in ethanol (3.6 mL) was refluxed for 
49 hours. Then, the reaction mixture was cooled to rt and filtered. The solvent was removed under reduced pressure. The residue was purified by flash chromatography on silica gel (petroleum: ethyl acetate $=4: 1$ ) provided give compound $(S)-\mathbf{4 c}$ : Colorless oil, $98.7 \mathrm{mg}, 69 \%$ yield. ${ }^{1} \mathrm{H}$ NMR $\left(400 \mathrm{MHz}, \mathrm{CDCl}_{3}\right) \delta 7.11(\mathrm{~d}, J=8.4 \mathrm{~Hz}$, 2H), $6.80(\mathrm{~d}, J=8.0 \mathrm{~Hz}, 2 \mathrm{H}), 4.58$ (sept, $J=6.0 \mathrm{~Hz}, 2 \mathrm{H}), 4.26$ (brs, 4H), 3.24 (s, 2H), 2.93-2.89 (m, 4H), 2.32-2.67 (m, 2H), 2.06-1.98 (m, 2H), 1.35 (d, $J=6.4 \mathrm{~Hz}, 6 \mathrm{H})$, $1.34(\mathrm{~d}, J=6.4 \mathrm{~Hz}, 6 \mathrm{H}) ;{ }^{13} \mathrm{C} \mathrm{NMR}\left(100 \mathrm{MHz}, \mathrm{CDCl}_{3}\right) \delta 156.7,149.4,135.3,126.0$, 124.4, 112.6, 71.0, 61.8, 57.3, 40.0, 29.8, 22.3; IR (thin film): $v_{\max }\left(\mathrm{cm}^{-1}\right)=3437,2974$, 2936, 1592, 1471, 1278, 1114, 1018, 807; HRMS (EI) calcd for $\mathrm{C}_{25} \mathrm{H}_{30} \mathrm{O}_{3}\left[\mathrm{M}-\mathrm{H}_{2} \mathrm{O}\right]^{+}$: 378.2195; Found: $378.2189 ;[\alpha]_{\mathrm{D}}^{29}=-101.9\left(\mathrm{c}=1.0, \mathrm{CHCl}_{3}\right)$.

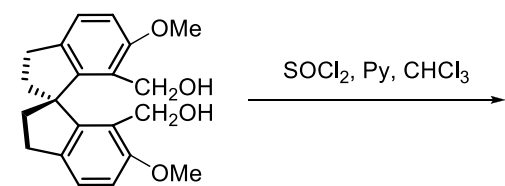

(S) $-4 a$

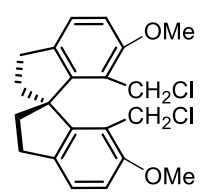

(S)-5a

To a solution of $(S)-4 a(368 \mathrm{mg}, 1.08 \mathrm{mmol}, 1$ equiv.) and pyridine (87 $\mu \mathrm{L}, 1.08$ mmol, 1 equiv.) in $\mathrm{CHCl}_{3}(10 \mathrm{~mL})$ was added a solution of freshly distilled $\mathrm{SOCl}_{2}$ (0.78 mL, $10.8 \mathrm{mmol}, 10$ equiv.) in $\mathrm{CHCl}_{3}(5 \mathrm{~mL})$ at $0{ }^{\circ} \mathrm{C}$. After the addition, the reaction mixture was stirred at $0^{\circ} \mathrm{C}$ for $30 \mathrm{~min}$ and then heated at reflux for 40 hours. After cooling to room temperature, the reaction was then quenched by slow addition of water $(10 \mathrm{~mL})$. The aqueous layer was separated and extracted with $\mathrm{CHCl}_{3}(10 \mathrm{~mL})$ three times. The combined organic layers were washed with saturated aqueous $\mathrm{NaHCO}_{3}$ and brine, dried over anhydrous $\mathrm{Na}_{2} \mathrm{SO}_{4}$, filtered and concentrated under reduced pressure. The residue was purified by column chromatography on silica gel with ethyl acetate/DCM/petroleum ether $=1: 2: 100$ to afford $(S)-5 \mathbf{a}(352.4 \mathrm{mg}, 86 \%$ yield) as a white solid.

White solid, m.p. $=202-204{ }^{\circ} \mathrm{C} .{ }^{1} \mathrm{H}$ NMR $\left(400 \mathrm{MHz}, \mathrm{CDCl}_{3}\right) \delta 7.23(\mathrm{~d}, J=8.0 \mathrm{~Hz}$, $2 \mathrm{H}), 6.83(\mathrm{~d}, J=8.0 \mathrm{~Hz}, 2 \mathrm{H}), 4.38(\mathrm{~d}, J=10.8 \mathrm{~Hz}, 2 \mathrm{H}), 4.17(\mathrm{~d}, J=10.8 \mathrm{~Hz}, 2 \mathrm{H})$, $3.88(\mathrm{~s}, 6 \mathrm{H}), 3.00-2.96(\mathrm{~m}, 4 \mathrm{H}), 2.41-2.31(\mathrm{~m}, 4 \mathrm{H}) ;{ }^{13} \mathrm{C} \mathrm{NMR}\left(100 \mathrm{MHz}, \mathrm{CDCl}_{3}\right) \delta$ 158.0, 149.1, 135.6, 125.8, 122.1, 110.4, 62.3, 56.1, 39.2, 38.4, 29.8; IR (thin film): 
$v_{\max }\left(\mathrm{cm}^{-1}\right)=3005,2939,1589,1286,1087,807$; HRMS (EI) calcd for $\mathrm{C}_{21} \mathrm{H}_{22} \mathrm{O}_{2} \mathrm{Cl}_{2}$ $[\mathrm{M}]^{+}:$376.0997; Found: 376.1002; $[\alpha]_{\mathrm{D}}^{28}=-145.0\left(\mathrm{c}=0.2, \mathrm{CHCl}_{3}\right)$.

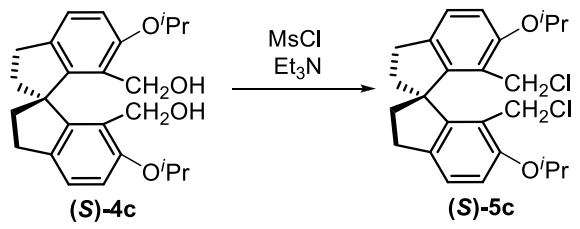

To a stirred solution of $(S)-\mathbf{4 c}\left(96.5 \mathrm{mg}, 0.243 \mathrm{mmol}, 1\right.$ equiv.) and $\mathrm{Et}_{3} \mathrm{~N}(0.2 \mathrm{~mL}$, 1.46 mmol, 6 equiv.) in $\mathrm{CH}_{2} \mathrm{Cl}_{2}(2.4 \mathrm{~mL})$ at $0{ }^{\circ} \mathrm{C}$, methanesulfonyl chloride $(113 \mu \mathrm{L}$, $1.46 \mathrm{mmol}, 6$ equiv.) was added dropwise over $15 \mathrm{~min}$. After the addition, the reaction mixture was stirred at $0{ }^{\circ} \mathrm{C}$ for $30 \mathrm{~min}$ and then at $\mathrm{rt}$ for $15 \mathrm{~h}$. The reaction mixture was washed with $\mathrm{H}_{2} \mathrm{O}(10 \mathrm{~mL})$, and the organic layer was separated, dried over $\mathrm{Na}_{2} \mathrm{SO}_{4}$, filtered and concentrated under reduced pressure. The residue was purified by column chromatography on silica gel with ethyl acetate/petroleum ether $=$ 1:50 to afford $(\boldsymbol{S})-\mathbf{5 c}(51 \mathrm{mg}, 48 \%$ yield).

Colorless oil. ${ }^{1} \mathrm{H}$ NMR $\left(400 \mathrm{MHz}, \mathrm{CDCl}_{3}\right) \delta 7.18(\mathrm{~d}, J=8.4 \mathrm{~Hz}, 2 \mathrm{H}), 6.81(\mathrm{~d}, J=8.4$ $\mathrm{Hz}, 2 \mathrm{H}), 4.58$ (sept, $J=6.0 \mathrm{~Hz}, 2 \mathrm{H}), 4.37$ (d, $J=10.4 \mathrm{~Hz}, 2 \mathrm{H}), 4.11(\mathrm{~d}, J=10.4 \mathrm{~Hz}$, 2H), 3.02-2.87 (m, 4H), 2.44-2.36 (m, 2H), 2.34-2.22 (m, 2H), $1.35(\mathrm{~d}, J=5.6 \mathrm{~Hz}$, $6 \mathrm{H}), 1.33(\mathrm{~d}, J=5.6 \mathrm{~Hz}, 6 \mathrm{H}) ;{ }^{13} \mathrm{C} \mathrm{NMR}\left(100 \mathrm{MHz}, \mathrm{CDCl}_{3}\right) \delta 156.3,149.3,135.2$, 125.6, 123.1, 112.5, 70.6, 62.4, 39.2, 38.5, 29.9, 22.2, 22.1; IR (thin film): $v_{\max }\left(\mathrm{cm}^{-1}\right)$ $=2975,2932,1588,1469,1250,1112,807,676$; Anal. calcd for $\mathrm{C}_{25} \mathrm{H}_{30} \mathrm{O}_{2} \mathrm{Cl}_{2}: \mathrm{C}$, 69.28; H, 6.98; Found: C, 69.07; H, 7.11; $[\alpha]_{\mathrm{D}}^{29}=-143.4\left(\mathrm{c}=0.50, \mathrm{CHCl}_{3}\right)$.

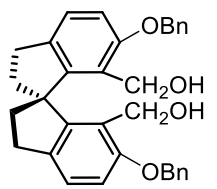

(S)-4b

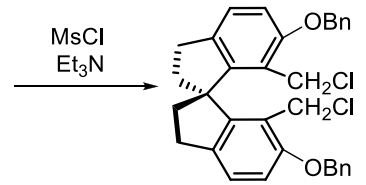

(S)-5b

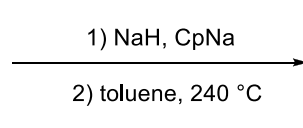

2) toluene, $240{ }^{\circ} \mathrm{C}$

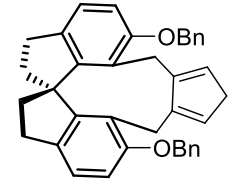

(S)-6b

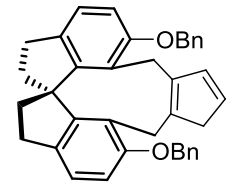

(S)-6b'

$(S)$-5b was prepared by the same procedure as the synthesis of $(S)$-5c from $(S)-\mathbf{4 c}$ $(98.5 \mathrm{mg}, 0.2 \mathrm{mmol})$. After general work up procedure, the reaction mixture was cooled to rt and extracted with EtOAc, washed with brine, dried over $\mathrm{Na}_{2} \mathrm{SO}_{4}$, concentrated under reduced pressure, and filtered through short silica gel column (ethyl acetate/petroleum ether $=1: 50$ ). The filtrate was concentrated and the residue was dissolved in THF $(2 \mathrm{~mL})$. To a cold $\left(0^{\circ} \mathrm{C}\right)$ suspension of sodium hydride $(9.1 \mathrm{mg}$, 
0.227 mmol, 1.14 equiv. $)$ in THF (1 mL) was added a solution of sodium cyclopentadienylide $(0.12 \mathrm{~mL}, 0.227 \mathrm{mmol}, 2.0 \mathrm{M}$ in THF, 1.14 equiv.). Then the crude $(S)$-5b in THF was added into the above mixture, followed by the addition of 15-crown-5 (64 $\mu \mathrm{L}, 0.324 \mathrm{mmol})$. The mixture was then heated at $80^{\circ} \mathrm{C}$ and stirred for 41 hours. After cooling to rt, the mixture was quenched with saturated $\mathrm{NH}_{4} \mathrm{Cl}$ (aq.) $(10$ $\mathrm{mL})$ and was extracted with ether $(3 \mathrm{X} 10 \mathrm{~mL})$. The organic layers were dried over $\mathrm{Na}_{2} \mathrm{SO}_{4}$, filtered and concentrated under reduced pressure. Purification of the residue by chromatography on silica gel (ethyl acetate/petroleum ether $=1: 100$ ) afforded a mixture. The mixture was dissolved in degassed toluene $(12.0 \mathrm{~mL})$ and stirred at $240^{\circ} \mathrm{C}$ for 16 hours in a sealed tube. After cooling down to rt, the solvent was evaporated under reduced pressure and the residue was purified by column chromatography on silica gel (ethyl acetate/petroleum ether $=1: 100)$ affording $49.8 \mathrm{mg}(48 \%$ yield $)$ of $(S)-\mathbf{6 b}+\mathbf{6} \mathbf{b}$ '.

White solid, 6b:6b' $=1.5: 1 .{ }^{1} \mathrm{H}$ NMR $\left(400 \mathrm{MHz} \mathrm{CDCl}_{3}\right) \delta$ 7.58-7.23 (m, 10H), 7.02-6.99 (m, 2H), 6.72-6.69 (m, 2H), $6.26(\mathrm{~d}, J=4.4 \mathrm{~Hz}, 1 \mathrm{H}), 5.95-5.93(\mathrm{~m}, 2 \mathrm{H})$, $4.96(\mathrm{~s}, 4 \mathrm{H}), 3.74(\mathrm{~d}, J=13.2 \mathrm{~Hz}, 1 \mathrm{H}), 3.56(\mathrm{dd}, J=13.2,5.6 \mathrm{~Hz}, 1 \mathrm{H}), 3.40$ (d, $J=$ $12.8 \mathrm{~Hz}, 1 \mathrm{H}), 3.33-3.25(\mathrm{~m}, 1 \mathrm{H}), 3.11-3.05(\mathrm{~m}, 0.58 \mathrm{H}), 2.98-2.88(\mathrm{~m}, 2 \mathrm{H}), 2.83-2.77$ (m, 2H), 2.69 (s, 1.42H), 2.29-2.24 (m, 2H), 2.05-1.97 (m, 2H); ${ }^{13} \mathrm{C}$ NMR (100 MHz, $\left.\mathrm{CDCl}_{3}\right) \delta 156.4,155.5,155.4,149.1,148.8,144.3,141.0$ 139.2, 138.8, 137.7, 137.6, $137.4,134.9,134.5,134.4,129.2,128.4,128.2,127.6,127.5,127.46,127.4,127.2$, 124.0, 123.4, 122.9, 122.8, 122.04, 122.96, 110.7, 110.4, 70.2, 70.1, 70.0, 62.2, 61.1, $47.2,39.3,39.2,38.7,38.1,29.4,29.2,26.6,24.6,24.2$; IR (thin film): $v_{\max }\left(\mathrm{cm}^{-1}\right)=$ 3085, 3061, 3030, 2924, 2846, 1586, 1453, 695; HRMS (ESI) calcd for $\mathrm{C}_{38} \mathrm{H}_{35} \mathrm{O}_{2}$ $[\mathrm{M}+\mathrm{H}]^{+}:$523.2632; Found: 523.2632; $[\alpha]_{\mathrm{D}}^{29}=+41.2\left(\mathrm{c}=0.20, \mathrm{CHCl}_{3}\right)$.

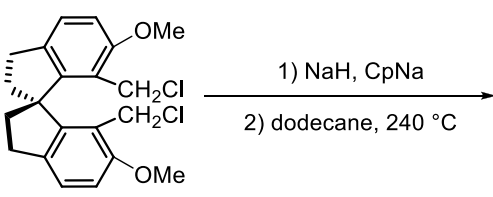

(S)-5a

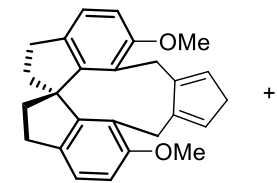

(S)-6a

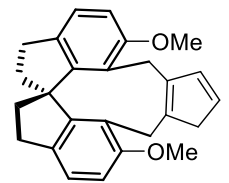

(S)-6a'

To a cold $\left(0{ }^{\circ} \mathrm{C}\right)$ suspension of sodium hydride (16.8 $\mathrm{mg}, 0.42 \mathrm{mmol}, 1.4$ equiv.) in THF $(1 \mathrm{~mL})$ was added a solution of sodium cyclopentadienylide $(0.21 \mathrm{~mL}, 0.42$ mmol, 2.0 M in THF, 1.4 equiv.). Then $(\boldsymbol{S})-5 \mathbf{a}(113.2 \mathrm{mg}, 0.3 \mathrm{mmol})$ in THF (2 mL) 
was added into the above mixture, followed by the addition of 15 -crown-5 (132.2 $\mu \mathrm{L}$, $0.6 \mathrm{mmol}, 2.0$ equiv.). The mixture was then heated at $80^{\circ} \mathrm{C}$ for 48 hours. After cooling to $\mathrm{rt}$, the mixture was quenched with saturated $\mathrm{NH}_{4} \mathrm{Cl}$ (aq.) $(10 \mathrm{~mL})$ and was extracted with ether $(3 \mathrm{X} 10 \mathrm{~mL})$. The organic layers were dried over $\mathrm{Na}_{2} \mathrm{SO}_{4}$, filtered and concentrated under reduced pressure. Purification of the residue by chromatography on silica gel (ethyl acetate/petroleum ether $=1: 100$ ) afforded a mixture. The mixture was dissolved in degassed dodecane $(30 \mathrm{~mL})$ and stirred at $240^{\circ} \mathrm{C}$ for 15 hours in a sealed tube. After cooling down to rt, the mixture was purified by column chromatography on silica gel (ethyl acetate/petroleum ether $=1: 100)$ affording $100.4 \mathrm{mg}(90 \%$ yield $)$ of $(S)-6 \mathbf{a}+6 \mathbf{a}^{\prime}$.

White solid, 6a:6a' $=1.3: 1 .{ }^{1} \mathrm{H}$ NMR $\left(400 \mathrm{MHz}, \mathrm{CDCl}_{3}\right) \delta$ 7.13-7.11 (m, 2H), 6.79-6.75 (m, 2H), $6.50(\mathrm{~d}, J=4.8 \mathrm{~Hz}, 0.43 \mathrm{H}), 6.15-6.12(\mathrm{~m}, 1.57 \mathrm{H}), 3.78-3.75(\mathrm{~m}$, 7H), 3.61-3.56 (m, 1H), $3.45(\mathrm{~d}, J=13.2 \mathrm{~Hz}, 1 \mathrm{H}), 3.38-3.29(\mathrm{~m}, 1 \mathrm{H}), 3.12-2.75(\mathrm{~m}$, $6 \mathrm{H}), 2.45-2.25(\mathrm{~m}, 2 \mathrm{H}), 2.13-2.05(\mathrm{~m}, 2 \mathrm{H}) ;{ }^{13} \mathrm{C} \mathrm{NMR}\left(100 \mathrm{MHz}, \mathrm{CDCl}_{3}\right) \delta$ 157.5, $156.5,156.4,148.8,148.6,144.4,141.0,139.0,138.5,134.7,134.24,134.19,128.7$, $127.5,124.0,123.2$, 122.73, 122.65, 122.0, 121.9, 110.0, 109.7, 109.2, 62.1, 61.5, $55.9,55.3,47.1,39.2,39.1,38.8,38.1,29.4,29.2,26.4,24.4,24.0$; IR (thin film): $v_{\max }\left(\mathrm{cm}^{-1}\right)=2947,2922,2850,1602,1476,1459,1251,1082,797$; HRMS (EI) calcd for $\mathrm{C}_{26} \mathrm{H}_{26} \mathrm{O}_{2}[\mathrm{M}]^{+}:$370.1933; Found: $370.1924 ;[\alpha]_{\mathrm{D}}{ }^{27}=+156.9\left(\mathrm{c}=0.20, \mathrm{CHCl}_{3}\right)$.

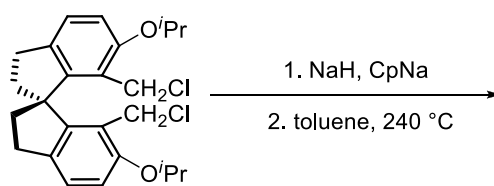

(S)-5c

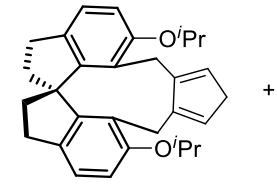

(S)-6c

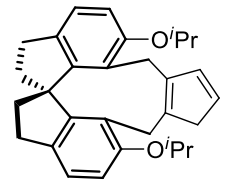

$(S)-6 c^{\prime}$

$(S)-\mathbf{6 c}+\mathbf{6} \mathbf{c}^{\prime}$ was prepared by the same procedure as the synthesis of $(S)-\mathbf{6} \mathbf{a}+\mathbf{6 a} \mathbf{a}$, except that toluene was used instead of dodecane.

White solid, $69.4 \mathrm{mg}, 92 \%$ yield $\left(0.177 \mathrm{mmol}\right.$ scale), $\mathbf{6 c}: 6 \mathbf{c}^{\prime}=1.2: 1 .{ }^{1} \mathrm{H}$ NMR (400 $\left.\mathrm{MHz}, \mathrm{CDCl}_{3}\right) \delta$ 7.06-6.93 (m, 2H), 6.68-6.65 (m, 2H), $6.39(\mathrm{~d}, J=5.2 \mathrm{~Hz}, 0.42 \mathrm{H})$, 6.01-5.99 (m, 1.44H), 4.51-4.42 (m, 2H), $3.63(\mathrm{~d}, J=13.2 \mathrm{~Hz}, 1 \mathrm{H}), 3.46(\mathrm{dd}, J=13.2$, $4.4 \mathrm{~Hz}, 1 \mathrm{H}), 3.39-3.10(\mathrm{~m}, 3 \mathrm{H}), 2.98-2.86(\mathrm{~m}, 2 \mathrm{H}), 2.84-2.73(\mathrm{~m}, 2 \mathrm{H}), 2.70(\mathrm{~s}, 1 \mathrm{H})$, 2.34-2.16 (m, 2H), 2.02-1.94 (m, 2H), 1.44-0.96 (m, 12H); ${ }^{13} \mathrm{C}$ NMR (100 MHz, 
$\left.\mathrm{CDCl}_{3}\right) \delta 155.4,154.5,154.4,149.2,148.9,148.8,144.6,141.0,139.2,139.16,134.1$, 133.8, 133.6, 128.7, 126.8, 124.6, 124.2, 123.7, 122.5, 121.8, 121.7, 111.4, 111.3, $69.3,69.2,62.2,61.7,47.2,39.2,39.1,38.8,37.8,29.5,29.2,26.6,24.7,24.3,22.3$, 22.2, 22.13, 22.1, 21.7, 21.6; IR (thin film): $v_{\max }\left(\mathrm{cm}^{-1}\right)=2974,2939,2875,2852$, 1589, 1466, 1246, 1114, 752; HRMS (ESI) calcd for $\mathrm{C}_{30} \mathrm{H}_{25} \mathrm{O}_{2}[\mathrm{M}+\mathrm{H}]^{+}$: 427.2632; Found: $427.2633 ;[\alpha]_{\mathrm{D}}^{29}=+105.9\left(\mathrm{c}=0.30, \mathrm{CHCl}_{3}\right)$. 


\section{Synthesis of Rh complexes:}

General procedure for the synthesis of Rh complex $[(S)-\mathbf{K 3 a}]$ was identical as the previous report on the preparation of chiral $\mathrm{CpRh}(\mathrm{I})$ complexes: ${ }^{4}$

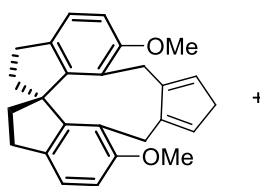

(S)-6a

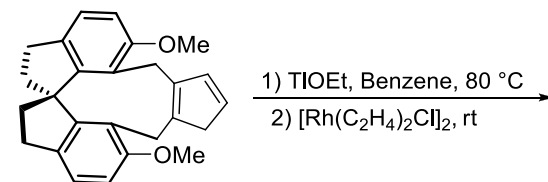

(S)-6a'

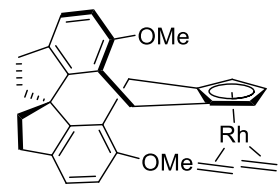

(S)-K3a

To a solution of $(S)-\mathbf{6 a}+\mathbf{6 a} \mathbf{a}^{\prime}(198 \mathrm{mg}, 287 \mu \mathrm{mol})$ in degassed benzene $(1.0 \mathrm{~mL})$ at $\mathrm{rt}$ was added a solution of thallium(I) ethanolate $(89.8 \mathrm{mg}, 0.36 \mathrm{mmol}, 1.20$ equiv.) in benzene $(2.0 \mathrm{~mL})$. The mixture was stirred for 16 hours at $80^{\circ} \mathrm{C}$ under dark. After cooling down to rt, $\left[\mathrm{Rh}\left(\mathrm{C}_{2} \mathrm{H}_{4}\right)_{2} \mathrm{Cl}\right]_{2}$ (70 mg, $0.18 \mathrm{mmol}, 0.60$ equiv.) was added. After stirring for 24 hours, the mixture was filtered through a pad of celite and neutral aluminum oxide. The yellow filtrate was concentrated under reduced pressure to afford $117.8 \mathrm{mg}$ ( $74 \%$ yield) of the desired complex $[(S)-\mathbf{K 3} \mathbf{a}]$.

Yellow solid, m.p. $=181-183{ }^{\circ} \mathrm{C}($ decomposed $) .{ }^{1} \mathrm{H}$ NMR $\left(400 \mathrm{MHz}, \mathrm{C}_{6} \mathrm{D}_{6}\right) \delta 7.04(\mathrm{~d}$, $J=8.4 \mathrm{~Hz}, 1 \mathrm{H}), 6.96(\mathrm{~d}, J=8.0 \mathrm{~Hz}, 1 \mathrm{H}), 6.60(\mathrm{~d}, J=8.4 \mathrm{~Hz}, 1 \mathrm{H}), 6.45(\mathrm{~d}, J=8.0 \mathrm{~Hz}$, 1H), $5.74(\mathrm{~s}, 1 \mathrm{H}), 4.60(\mathrm{~s}, 1 \mathrm{H}), 4.55(\mathrm{t}, J=2.4 \mathrm{~Hz}, 1 \mathrm{H}), 3.91(\mathrm{~d}, J=13.6 \mathrm{~Hz}, 1 \mathrm{H})$, $3.56(\mathrm{~s}, 3 \mathrm{H}), 3.44(\mathrm{~d}, J=13.6 \mathrm{~Hz}, 1 \mathrm{H}), 3.33(\mathrm{~s}, 3 \mathrm{H}), 3.29(\mathrm{~d}, J=12.8 \mathrm{~Hz}, 1 \mathrm{H}), 3.10(\mathrm{~d}$, $J=12.8 \mathrm{~Hz}, 1 \mathrm{H}), 2.91(\mathrm{t}, J=9.6 \mathrm{~Hz}, 2 \mathrm{H}), 2.81-2.72(\mathrm{~m}, 2 \mathrm{H}), 2.65-2.59(\mathrm{~m}, 2 \mathrm{H}), 2.13$ (t, $J=9.6 \mathrm{~Hz}, 2 \mathrm{H}), 2.08-1.91(\mathrm{~m}, 4 \mathrm{H}), 1.21(\mathrm{t}, J=9.6 \mathrm{~Hz}, 2 \mathrm{H}), 0.89(\mathrm{t}, J=9.6 \mathrm{~Hz}$, $2 \mathrm{H}) ;{ }^{13} \mathrm{C}$ NMR $\left(151 \mathrm{MHz}, \mathrm{C}_{6} \mathrm{D}_{6}\right) \delta 158.5,157.5,149.1,149.0,134.8,133.5,125.8$, 123.3, 123.2, 123.1, 111.0 (d, $J=4.4 \mathrm{~Hz}), 110.0,109.1,97.6$ (d, $J=3.6 \mathrm{~Hz}), 91.3$ (d, $J=8.0 \mathrm{~Hz}), 89.4(\mathrm{~d}, J=4.2 \mathrm{~Hz}), 83.0(\mathrm{~d}, J=4.4 \mathrm{~Hz}), 62.6,55.4,55.2,39.8(\mathrm{~d}, J=$ 17.7 Hz), 39.7, 39.4, $37.0(\mathrm{~d}, J=13.7 \mathrm{~Hz}), 29.4,24.0,21.9$; IR (thin film): $v_{\max }\left(\mathrm{cm}^{-1}\right)$ $=3054,2923,2849,1590,1461,1281,1015,791 ;$ HRMS (MALDI-FT_DHB) calcd for $\mathrm{C}_{26} \mathrm{H}_{26} \mathrm{O}_{2} \mathrm{Rh}\left[\mathrm{M}-2\left(\mathrm{C}_{2} \mathrm{H}_{4}\right)+\mathrm{H}\right]^{+}: 473.0982$; Found: 473.0985; $[\alpha]_{\mathrm{D}}{ }^{28}=-217.9(\mathrm{c}=$ $\left.0.20, \mathrm{CH}_{2} \mathrm{Cl}_{2}\right)$.

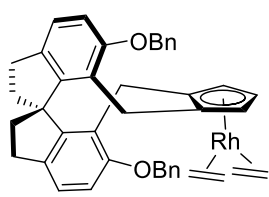

(S)-K3b 
Yellow gum, $49.7 \mathrm{mg}, 73 \%$ yield (0.1 mmol scale). ${ }^{1} \mathrm{H}$ NMR (400 MHz, $\left.\mathrm{C}_{6} \mathrm{D}_{6}\right) \delta 7.47$ $(\mathrm{d}, J=7.6 \mathrm{~Hz}, 2 \mathrm{H}), 7.28(\mathrm{~d}, J=7.6 \mathrm{~Hz}, 2 \mathrm{H}), 7.21(\mathrm{t}, J=7.6 \mathrm{~Hz}, 2 \mathrm{H}), 7.15-7.06(\mathrm{~m}$, 4H), $7.03(\mathrm{~d}, J=8.0 \mathrm{~Hz}, 1 \mathrm{H}), 6.95(\mathrm{~d}, J=8.0 \mathrm{~Hz}, 1 \mathrm{H}), 6.68(\mathrm{~d}, J=8.0 \mathrm{~Hz}, 1 \mathrm{H}), 6.54$ $(\mathrm{d}, J=8.0 \mathrm{~Hz}, 1 \mathrm{H}), 5.65$ (brs, $1 \mathrm{H}), 4.97\left(\mathrm{AB}, J_{A B}=12.0 \mathrm{~Hz}, 1 \mathrm{H}\right), 4.88\left(\mathrm{BA}, J_{B A}=\right.$ $12.0 \mathrm{~Hz}, 1 \mathrm{H}), 4.71$ (brs, 1H), 4.67 (dd, $J=15.2 \mathrm{~Hz}, 12.0 \mathrm{~Hz}, 2 \mathrm{H}), 4.32$ (t, $J=2.8 \mathrm{~Hz}$, 1H), $4.07(\mathrm{~d}, J=13.6 \mathrm{~Hz}, 1 \mathrm{H}), 3.54(\mathrm{~d}, J=13.6 \mathrm{~Hz}, 1 \mathrm{H}), 3.40(\mathrm{~d}, J=12.8 \mathrm{~Hz}, 1 \mathrm{H})$, $3.13(\mathrm{~d}, J=13.2 \mathrm{~Hz}, 1 \mathrm{H}), 2.84-2.58(\mathrm{~m}, 6 \mathrm{H}), 2.13-1.94(\mathrm{~m}, 6 \mathrm{H}), 1.18-1.12(\mathrm{~m}, 2 \mathrm{H})$, 0.82-0.76 (m, 2H); ${ }^{13} \mathrm{C}$ NMR (100 MHz, $\left.\mathrm{C}_{6} \mathrm{D}_{6}\right) \delta 157.6,156.6,149.13,149.09,138.6$, $137.8,134.9,133.7,128.6,128.5,127.9,127.6$, 127.0, 126.6, 123.4, 123.2, 123.1, $111.9(\mathrm{~d}, J=4.2 \mathrm{~Hz}), 111.2,110.7,97.0(\mathrm{~d}, J=3.8 \mathrm{~Hz}), 91.7(\mathrm{~d}, J=3.5 \mathrm{~Hz}), 89.6(\mathrm{~d}$, $J=4.2 \mathrm{~Hz}), 82.6(\mathrm{~d}, J=4.2 \mathrm{~Hz}), 70.4,69.9,62.7,40.1(\mathrm{~d}, J=14.7 \mathrm{~Hz}), 39.9,39.1$, $37.5(\mathrm{~d}, J=17.6 \mathrm{~Hz}), 30.2,29.4,24.1,22.3$; IR (thin film): $v_{\max }\left(\mathrm{cm}^{-1}\right)=3055,2924$, 2851, 1588, 1497, 1474, 1260, 1104, 803, 695; HRMS (MALDI-FT_DHB) calcd for $\mathrm{C}_{38} \mathrm{H}_{33} \mathrm{O}_{2} \mathrm{Rh}\left[\mathrm{M}-2\left(\mathrm{C}_{2} \mathrm{H}_{4}\right)+\mathrm{H}\right]^{+}:$624.1530; Found: 624.1531; $[\alpha]_{\mathrm{D}}{ }^{28}=-223.2(\mathrm{c}=0.20$, $\mathrm{CH}_{2} \mathrm{Cl}_{2}$ ).

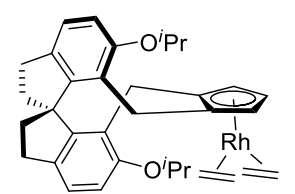

(S)-K3c

Yellow solid, $42.5 \mathrm{mg}, 73 \%$ yield (0.1 mmol scale), m.p. > $300{ }^{\circ} \mathrm{C} .{ }^{1} \mathrm{H}$ NMR (400 $\left.\mathrm{MHz}, \mathrm{C}_{6} \mathrm{D}_{6}\right) \delta 7.02(\mathrm{~d}, J=8.4 \mathrm{~Hz}, 1 \mathrm{H}), 6.98(\mathrm{~d}, J=8.0 \mathrm{~Hz}, 1 \mathrm{H}), 6.72(\mathrm{~d}, J=8.4 \mathrm{~Hz}$, $1 \mathrm{H}), 6.51(\mathrm{~d}, J=8.4 \mathrm{~Hz}, 1 \mathrm{H}), 5.88$ (brs, $1 \mathrm{H}), 4.76$ (brs, $1 \mathrm{H}), 4.43$ (t, $J=2.4 \mathrm{~Hz}, 1 \mathrm{H})$, 4.27 (sept, $J=6.0 \mathrm{~Hz}, 1 \mathrm{H}), 4.19$ (sept, $J=6.4 \mathrm{~Hz}, 1 \mathrm{H}), 3.91(\mathrm{~d}, J=13.4 \mathrm{~Hz}, 1 \mathrm{H}$ ), $3.47(\mathrm{~d}, J=13.2 \mathrm{~Hz}, 1 \mathrm{H}), 3.39(\mathrm{~d}, J=13.2 \mathrm{~Hz}, 1 \mathrm{H}), 3.13(\mathrm{~d}, J=12.8 \mathrm{~Hz}, 1 \mathrm{H})$, 2.91-2.86 (m, 2H), 2.81-2.70 (m, 2H), 2.66-2.59 (m, 2H), 2.22-2.18 (m, 2H), 2.10-1.89 (m, 4H), $1.40(\mathrm{~d}, J=6.0 \mathrm{~Hz}, 3 \mathrm{H}), 1.36-1.30(\mathrm{~m}, 2 \mathrm{H}), 1.16-1.10(\mathrm{~m}, 9 \mathrm{H})$, 0.94-0.91 (m, 2H); ${ }^{13} \mathrm{C}$ NMR (100 MHz, $\left.\mathrm{C}_{6} \mathrm{D}_{6}\right) \delta 157.3,155.3,149.33,149.29,134.1$, 133.5, 128.6, 123.5, 123.4, 123.2, 113.5, $112.4(\mathrm{~d}, J=4.2 \mathrm{~Hz}), 111.4,97.2(\mathrm{~d}, J=3.8$ Hz), 91.7 (d, $J=3.5 \mathrm{~Hz}), 89.5$ (d, $J=4.4 \mathrm{~Hz}), 81.9$ (d, $J=4.4 \mathrm{~Hz}), 70.9,69.1,62.9$, 40.0, 39.9 (d, $J=10.3 \mathrm{~Hz}), 39.1,38.2$ (d, $J=10.6 \mathrm{~Hz}), 29.5,29.4,24.2,23.3,22.6$, 
22.2, 22.1; IR (thin film): $v_{\max }\left(\mathrm{cm}^{-1}\right)=3054,2964,2927,1587,1469,1432,1381$, 1259, 1117, 806; HRMS (MALDI-FT_DHB) calcd for $\mathrm{C}_{30} \mathrm{H}_{34} \mathrm{O}_{2} \mathrm{Rh}\left[\mathrm{M}-2\left(\mathrm{C}_{2} \mathrm{H}_{4}\right)+\mathrm{H}\right]^{+}$: 529.1608; Found: 529.1608; $[\alpha]_{\mathrm{D}}^{25}=-284.5\left(\mathrm{c}=0.20, \mathrm{CH}_{2} \mathrm{Cl}_{2}\right)$.

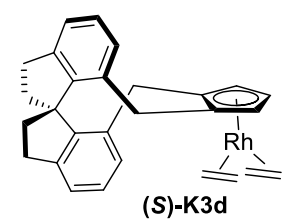

Yellow solid, $50.1 \mathrm{mg}, 67 \%$ yield (0.16 mmol scale), m.p. $=127-128{ }^{\circ} \mathrm{C} .{ }^{1} \mathrm{H}$ NMR $\left(400 \mathrm{MHz}, \mathrm{C}_{6} \mathrm{D}_{6}\right) \delta 7.59(\mathrm{~d}, J=7.2 \mathrm{~Hz}, 1 \mathrm{H}), 7.26(\mathrm{t}, J=7.6 \mathrm{~Hz}, 1 \mathrm{H}), 7.10-7.02(\mathrm{~m}$, 2H), 6.99 (d, $J=6.8 \mathrm{~Hz}, 1 \mathrm{H}), 6.89(\mathrm{~d}, J=7.2 \mathrm{~Hz}, 1 \mathrm{H}), 5.14$ (brs, 1H), 4.69 (t, $J=2.4$ $\mathrm{Hz}, 1 \mathrm{H}), 4.07$ (brs, 1H), 3.45 (d, $J=14.0 \mathrm{~Hz}, 1 \mathrm{H}), 3.07$ (dd, $J=13.2,6.4 \mathrm{~Hz}, 2 \mathrm{H})$, 2.91-2.86 (m, 2H), 2.81-2.73 (m, 2H), 2.67-2.59 (m, 2H), 2.49 (d, $J=13.2 \mathrm{~Hz}, 1 \mathrm{H})$, 2.08-1.79 (m, 6H), 1.34-1.21 (m, 2H), 0.97-0.88 (m, 2H); ${ }^{13} \mathrm{C}$ NMR (100 MHz, $\left.\mathrm{C}_{6} \mathrm{D}_{6}\right)$ $\delta 147.7,147.3,143.2,141.8,138.1,134.5,132.6,128.8,127.5,123.1,123.07,111.3$ $(\mathrm{d}, J=4.8 \mathrm{~Hz}), 101.0(\mathrm{~d}, J=3.7 \mathrm{~Hz}), 89.8(\mathrm{~d}, J=3.8 \mathrm{~Hz}), 89.0(\mathrm{~d}, J=4.0 \mathrm{~Hz}), 83.6$ (d, $J=4.1 \mathrm{~Hz}), 61.6,41.5(\mathrm{~d}, J=12.3 \mathrm{~Hz}), 39.3,38.8,36.7$ (d, $J=13.5 \mathrm{~Hz}), 30.4$, 30.3, 30.2, 27.2; IR (thin film): $v_{\max }\left(\mathrm{cm}^{-1}\right)=3048,2983,2949,2837,1593,1429,755$; Anal. calcd for $\mathrm{C}_{28} \mathrm{H}_{29} \mathrm{Rh}$ : C, 71.79; H, 6.24; Found: C, 72.22; H, 6.36; $[\alpha]_{\mathrm{D}}^{29}=-172.4$ (c = 0.20, $\mathrm{CHCl}_{3}$ ). 


\section{Synthesis and Characterization of Starting Materials}

General procedure for the preparation of the starting materials was identical as previously reported. ${ }^{5}$

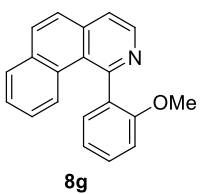

Yellow sticky oil, $0.74 \mathrm{~g}, 86 \%$ yield. ${ }^{1} \mathrm{H}$ NMR (400 MHz, $\left.\mathrm{CDCl}_{3}\right) \delta 8.67(\mathrm{~d}, J=4.4$ $\mathrm{Hz}, 1 \mathrm{H}), 7.87(\mathrm{~d}, J=8.4 \mathrm{~Hz}, 1 \mathrm{H}), 7.64(\mathrm{~d}, J=7.6 \mathrm{~Hz}, 2 \mathrm{H}), 7.43-7.31(\mathrm{~m}, 5 \mathrm{H})$, 7.17-7.6 (m, 2H), $6.86(\mathrm{~d}, J=7.2 \mathrm{~Hz}, 1 \mathrm{H}), 3.28(\mathrm{~s}, 3 \mathrm{H}) ;{ }^{13} \mathrm{C} \mathrm{NMR}\left(100 \mathrm{MHz}, \mathrm{CDCl}_{3}\right)$ $\delta 155.8,155.6,142.9,136.9,132.9,132.4,131.1,129.4,129.3,129.2,128.1,126.0$, $125.9,125.4,124.9,124.5,121.0,120.3,110.9,54.7$; IR (thin film): $v_{\max }\left(\mathrm{cm}^{-1}\right)=3056$, 2968, 2835, 1584, 1491, 1237, 1022, 750; HRMS (ESI) calcd for $\mathrm{C}_{20} \mathrm{H}_{16} \mathrm{NO}[\mathrm{M}+\mathrm{H}]^{+}$: 286.1226; Found: 286.1227.

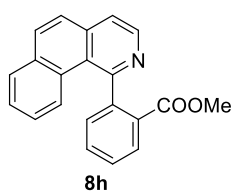

Yellow sticky oil, $0.19 \mathrm{~g}, 12 \%$ yield. ${ }^{1} \mathrm{H}$ NMR (400 MHz, $\left.\mathrm{CDCl}_{3}\right) \delta 8.67(\mathrm{~d}, J=5.2$ $\mathrm{Hz}, 1 \mathrm{H}), 8.24-8.14(\mathrm{~m}, 1 \mathrm{H}), 7.88(\mathrm{~d}, J=8.8 \mathrm{~Hz}, 1 \mathrm{H}), 7.82(\mathrm{~d}, J=8.0 \mathrm{~Hz}, 1 \mathrm{H})$, 7.70-7.66 (m, 2H), 7.64-7.50 (m, 3H), 7.46-7.43 (m, 1H), 7.33-7.27 (m, 1H), 7.22-7.17 (m, 1H), $3.43(\mathrm{~s}, 3 \mathrm{H}) ;{ }^{13} \mathrm{C} \mathrm{NMR}\left(100 \mathrm{MHz}, \mathrm{CDCl}_{3}\right) \delta 166.9,158.6,145.4$, 142.9, 137.6, 133.2, 132.7, 131.8, 131.0, 129.7, 129.4, 129.3, 128.7, 128.1, 126.7, 126.5, 125.4, 124.0, 120.6, 51.7; IR (thin film): $v_{\max }\left(\mathrm{cm}^{-1}\right)=3052,2952,2914,2848$, 1722, 1585, 1555, 1262, 853; HRMS (ESI) calcd for $\mathrm{C}_{21} \mathrm{H}_{16} \mathrm{NO}_{2}[\mathrm{M}+\mathrm{H}]^{+}: 314.1176$; Found: 314.1177 .

General procedure for asymmetric oxidative coupling reaction:
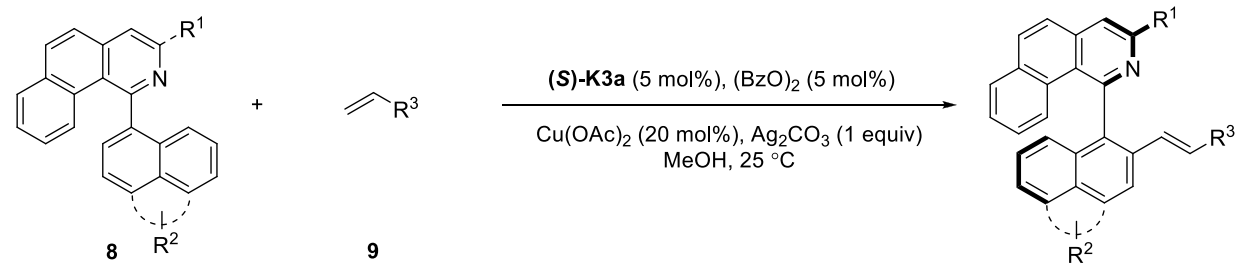

A flame-dried sealed tube was cooled to room temperature and filled with argon. To 
this tube were added $(S)-\mathbf{K 3 a}(10 \mu \mathrm{mol}, 5.3 \mathrm{mg}),(\mathrm{BzO})_{2}(10 \mu \mathrm{mol}, 2.4 \mathrm{mg})$, and $\mathrm{MeOH}(0.5 \mathrm{~mL})$. The mixture was stirred at room temperature. After $30 \mathrm{~min}$, biaryl compound $8(0.2 \mathrm{mmol})$, alkene $9(0.24 \mathrm{mmol}), \mathrm{Cu}(\mathrm{OAc})_{2}(0.04 \mathrm{mmol}, 7.3 \mathrm{mg})$, $\mathrm{Ag}_{2} \mathrm{CO}_{3}(0.2 \mathrm{mmol}, 55.2 \mathrm{mg})$ and $\mathrm{MeOH}(0.5 \mathrm{~mL})$ were added and the reaction mixture was stirred at $25{ }^{\circ} \mathrm{C}$ for indicated time. After then, saturated aqueous $\mathrm{NaHCO}_{3}$ solution $(5 \mathrm{~mL})$ and DCM $(5 \mathrm{~mL})$ were added. The organic layers were separated, and the aqueous layer was extracted with DCM $(2 \times 5 \mathrm{~mL})$. The combined organic layers were dried over $\mathrm{Na}_{2} \mathrm{SO}_{4}$, filtered, and concentrated under reduced pressure. The residue was purified by column chromatography on silica gel (petroleum ether/acetone $=30: 1$ ) to afford the coupling product.

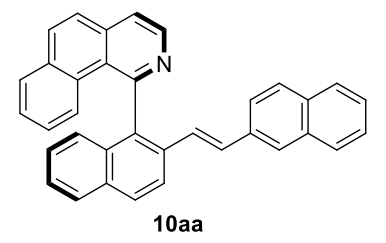

10aa. ${ }^{5} 84.6 \mathrm{mg}, 92 \%$ yield. ${ }^{1} \mathrm{H}$ NMR $\left(400 \mathrm{MHz}, \mathrm{CDCl}_{3}\right) \delta 8.94(\mathrm{~d}, J=4.8 \mathrm{~Hz}, 1 \mathrm{H})$, 8.11-8.04 (m, 2H), 8.01-7.73 (m, 5H), 7.67-7.52 (m, 5H), 7.42-7.32 (m, 4H), 7.24 (s, 1H), 7.21-7.13 (m, 1H), 7.13-6.93 (m, 3H), $6.77(\mathrm{~d}, J=16.4 \mathrm{~Hz}, 1 \mathrm{H})$; [Daicel Chiralcel AD-H $(0.46 \mathrm{~cm} \times 25 \mathrm{~cm}), n$-hexane/2-propanol $=90 / 10, v=1.0 \mathrm{~mL} \cdot \mathrm{min}^{-1}$, $\lambda=254 \mathrm{~nm}, \mathrm{t}($ minor $)=20.96 \mathrm{~min}, \mathrm{t}($ major $)=46.04 \mathrm{~min}], 95: 5 \mathrm{er} ;[\alpha]_{\mathrm{D}}^{22}=+560.1$ $\left(\mathrm{c}=0.22, \mathrm{CHCl}_{3}\right)$.

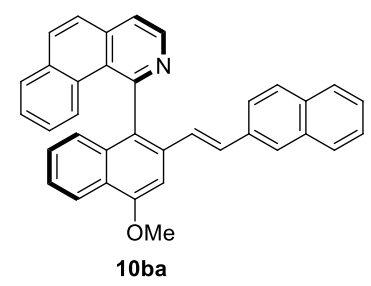

10ba. ${ }^{5} 78.3 \mathrm{mg}, 80 \%$ yield. ${ }^{1} \mathrm{H}$ NMR $\left(400 \mathrm{MHz}, \mathrm{CDCl}_{3}\right) \delta 8.92(\mathrm{~d}, J=5.2 \mathrm{~Hz}, 1 \mathrm{H})$, $8.35(\mathrm{~d}, J=8.4 \mathrm{~Hz}, 1 \mathrm{H}), 7.96(\mathrm{~d}, J=8.8 \mathrm{~Hz}, 1 \mathrm{H}), 7.90-7.76(\mathrm{~m}, 3 \mathrm{H}), 7.71(\mathrm{~d}, J=8.8$ $\mathrm{Hz}, 1 \mathrm{H})$, 7.67-7.64 (m, 2H), 7.54-7.53 (m, 2H), 7.45-7.31 (m, 5H), 7.21-7.17 (m, 2H), 7.13-7.00 (m, 3H), 6.78 (d, $J=16.4 \mathrm{~Hz}, 1 \mathrm{H}), 4.23$ (s, 3H); [Daicel Chiralpak ID-3 $(0.46 \mathrm{~cm} \times 15 \mathrm{~cm}), n$-hexane/2-propanol $=70 / 30, v=0.7 \mathrm{~mL} \cdot \mathrm{min}^{-1}, \quad \lambda=214 \mathrm{~nm}, \mathrm{t}$ 
$($ minor $)=11.18 \mathrm{~min}, \mathrm{t}($ major $)=16.53 \mathrm{~min}], 97: 3 \mathrm{er} ;[\alpha]_{\mathrm{D}}^{28}=+485.5(\mathrm{c}=0.20$, $\left.\mathrm{CHCl}_{3}\right)$.

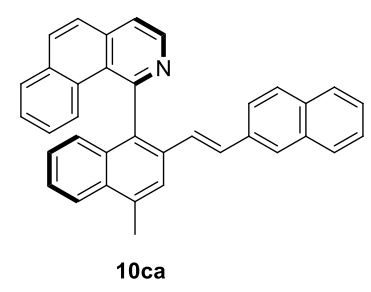

10ca. ${ }^{5} 86.4 \mathrm{mg}, 92 \%$ yield. ${ }^{1} \mathrm{H}$ NMR $\left(400 \mathrm{MHz}, \mathrm{CDCl}_{3}\right) \delta 8.92(\mathrm{~d}, J=5.2 \mathrm{~Hz}, 1 \mathrm{H})$, $8.07(\mathrm{~d}, J=8.4 \mathrm{~Hz}, 1 \mathrm{H}), 8.01-7.92(\mathrm{~m}, 2 \mathrm{H}), 7.89-7.84(\mathrm{~m}, 2 \mathrm{H}), 7.80(\mathrm{~d}, J=7.2 \mathrm{~Hz}$, 1H), 7.67-7.64 (m, 3H), 7.54-7.52 (m 2H), 7.47-7.41 (m, 1H), 7.39-7.32 (m, 3H), 7.24 $(\mathrm{d}, J=2.0 \mathrm{~Hz}, 1 \mathrm{H}), 7.20-7.17(\mathrm{~m}, 1 \mathrm{H}), 7.13-7.00(\mathrm{~m}, 3 \mathrm{H}), 6.74(\mathrm{~d}, J=16.4 \mathrm{~Hz}, 1 \mathrm{H})$, 2.90 (s, 3H); [Daicel Chiralcel AD-H $(0.46 \mathrm{~cm} \times 25 \mathrm{~cm}), n$-hexane/2-propanol = $90 / 10, v=1.0 \mathrm{~mL} \cdot \mathrm{min}^{-1}, \quad \lambda=254 \mathrm{~nm}, \mathrm{t}($ minor $)=14.86 \mathrm{~min}, \mathrm{t}($ major $\left.)=44.71 \mathrm{~min}\right]$, $92: 8 \mathrm{er} ;[\alpha]_{\mathrm{D}}{ }^{27}=+507.9\left(\mathrm{c}=0.20, \mathrm{CHCl}_{3}\right)$.

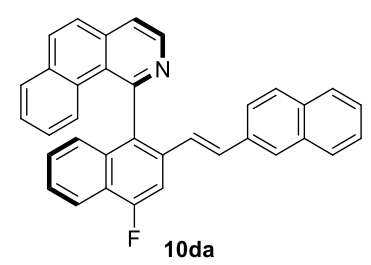

10da. ${ }^{5} 92.3 \mathrm{mg}, 97 \%$ yield. ${ }^{1} \mathrm{H}$ NMR $\left(400 \mathrm{MHz}, \mathrm{CDCl}_{3}\right) \delta 8.93(\mathrm{~d}, J=5.2 \mathrm{~Hz}, 1 \mathrm{H})$, $8.20(\mathrm{~d}, J=8.4 \mathrm{~Hz}, 1 \mathrm{H}), 8.00(\mathrm{~d}, J=8.8 \mathrm{~Hz}, 1 \mathrm{H}), 7.92(\mathrm{~d}, J=5.2 \mathrm{~Hz}, 1 \mathrm{H}), 7.88$ (d, $J$ $=8.8 \mathrm{~Hz}, 1 \mathrm{H}), 7.83(\mathrm{~d}, J=8.0 \mathrm{~Hz}, 1 \mathrm{H}), 7.78(\mathrm{~d}, J=12.0 \mathrm{~Hz}, 1 \mathrm{H}), 7.68-7.66(\mathrm{~m}, 2 \mathrm{H})$, $7.59(\mathrm{~d}, J=8.8 \mathrm{~Hz}, 1 \mathrm{H}), 7.55-7.53(\mathrm{~m}, 2 \mathrm{H}), 7.50-7.45(\mathrm{~m}, 1 \mathrm{H}), 7.44-7.33(\mathrm{~m}, 3 \mathrm{H})$, 7.26-7.19 (m, 2H), 7.12-7.05 (m, 3H), $6.72(\mathrm{dd}, J=16.0,1.6 \mathrm{~Hz}, 1 \mathrm{H})$; [Daicel Chiralcel AD-H $(0.46 \mathrm{~cm} \times 25 \mathrm{~cm}), n$-hexane/2-propanol $=90 / 10, v=1.0 \mathrm{~mL} \cdot \mathrm{min}^{-1}$, $\lambda=254 \mathrm{~nm}, \mathrm{t}($ minor $)=15.00 \mathrm{~min}, \mathrm{t}($ major $)=34.03 \mathrm{~min}], 95: 5 \mathrm{er} ;[\alpha]_{\mathrm{D}}^{27}=+495.0$ (c = 0.20, $\mathrm{CHCl}_{3}$ ).

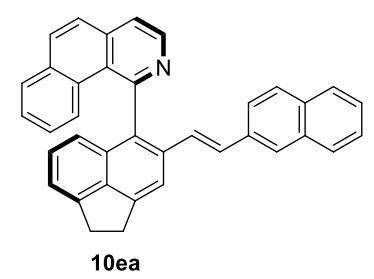


10ea. ${ }^{5} 73.9 \mathrm{mg}, 76 \%$ yield. ${ }^{1} \mathrm{H}$ NMR $\left(400 \mathrm{MHz}, \mathrm{CDCl}_{3}\right) \delta 8.91(\mathrm{~d}, J=5.2 \mathrm{~Hz}, 1 \mathrm{H})$, $7.98(\mathrm{~d}, J=8.8 \mathrm{~Hz}, 1 \mathrm{H}), 7.91(\mathrm{~s}, 1 \mathrm{H}), 7.87-7.84(\mathrm{~m}, 2 \mathrm{H}), 7.81-7.77(\mathrm{~m}, 2 \mathrm{H})$, 7.67-7.65 (m, 2H), $7.53(\mathrm{~d}, J=8.4 \mathrm{~Hz}, 1 \mathrm{H}), 7.50(\mathrm{~s}, 1 \mathrm{H}), 7.42-7.31(\mathrm{~m}, 3 \mathrm{H})$, 7.25-7.22 (m, 2H), 7.19-7.14 (m, 1H), 7.13-7.03 (m, 2H), $6.90(\mathrm{~d}, J=16.0 \mathrm{~Hz}, 1 \mathrm{H})$, $6.78(\mathrm{~d}, J=8.0 \mathrm{~Hz}, 1 \mathrm{H}), 3.62-3.44(\mathrm{~m}, 4 \mathrm{H})$; [Daicel Chiralpak ID-3 (0.46 cm x 15 $\mathrm{cm}$ ), $n$-hexane/2-propanol $=90 / 10, v=0.7 \mathrm{~mL} \cdot \mathrm{min}^{-1}, \lambda=254 \mathrm{~nm}, \mathrm{t}$ (minor) $=$ $19.65 \mathrm{~min}, \mathrm{t}($ major $)=68.04 \mathrm{~min}], 93: 7 \mathrm{er} ;[\alpha]_{\mathrm{D}}^{28}=+530.6\left(\mathrm{c}=0.20, \mathrm{CHCl}_{3}\right)$.

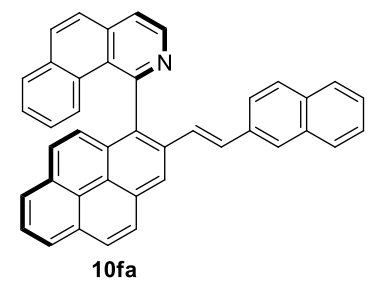

10fa. ${ }^{5} 87.1 \mathrm{mg}, 82 \%$ yield. ${ }^{1} \mathrm{H}$ NMR $\left(400 \mathrm{MHz}, \mathrm{CDCl}_{3}\right) \delta 8.98(\mathrm{~d}, J=5.2 \mathrm{~Hz}, 1 \mathrm{H})$, $8.77(\mathrm{~s}, 1 \mathrm{H}), 8.26-8.16(\mathrm{~m}, 3 \mathrm{H}), 8.08(\mathrm{~d}, J=7.6 \mathrm{~Hz}, 1 \mathrm{H}), 8.04-7.88(\mathrm{~m}, 4 \mathrm{H})$, 7.82-7.78 (m, 2H), 7.71-7.69 (m, 2H), 7.59-7.57 (m, 2H), 7.43-7.31 (m, 6H), 7.17 (d, $J=8.0 \mathrm{~Hz}, 1 \mathrm{H}), 7.00(\mathrm{~d}, J=16.0 \mathrm{~Hz}, 1 \mathrm{H}), 6.84(\mathrm{t}, J=8.4 \mathrm{~Hz}, 1 \mathrm{H})$; [Daicel Chiralcel $\mathrm{AD}-\mathrm{H}(0.46 \mathrm{~cm} \times 25 \mathrm{~cm}), n$-hexane $/ 2$-propanol $=70 / 30, v=1.0 \mathrm{~mL} \cdot \mathrm{min}^{-1}, \quad \lambda=254$ $\mathrm{nm}, \mathrm{t}($ minor $)=10.69 \mathrm{~min}, \mathrm{t}($ major $)=40.53 \mathrm{~min}], 82: 18 \mathrm{er} ;[\alpha]_{\mathrm{D}}{ }^{27}=+38.5(\mathrm{c}=0.20$, $\left.\mathrm{CHCl}_{3}\right)$.

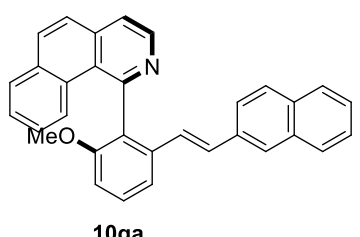

10ga. White foam, $74.4 \mathrm{mg}, 85 \%$ yield. ${ }^{1} \mathrm{H}$ NMR (400 MHz, $\left.\mathrm{CDCl}_{3}\right) \delta 8.84(\mathrm{~d}, J=$ $5.2 \mathrm{~Hz}, 1 \mathrm{H}), 7.92(\mathrm{~d}, J=8.8 \mathrm{~Hz}, 1 \mathrm{H}), 7.86(\mathrm{~d}, J=8.8 \mathrm{~Hz}, 1 \mathrm{H}), 7.78(\mathrm{~d}, J=7.6 \mathrm{~Hz}$, 1H), 7.73-7.69 (m, 2H), 7.64-7.55 (m, 3H), 7.61-7.57 (m, 4H), 7.34-7.22 (m, 3H), 7.15-7.04 (m, 2H), $6.96(\mathrm{~d}, J=8.0 \mathrm{~Hz}, 1 \mathrm{H}), 6.67$ (d, $J=16.4 \mathrm{~Hz}, 1 \mathrm{H}), 3.47$ (s, 3H); ${ }^{13} \mathrm{C}$ NMR $\left(100 \mathrm{MHz}, \mathrm{CDCl}_{3}\right) \delta 156.8,155.1,144.0,137.6,136.7,134.6,133.2,133.0$, $132.7,132.1,132.0,130.4,129.8,129.4,128.8,127.8,127.7,127.4,127.1,126.7$, $126.6,126.3,126.0,125.95,125.66,125.64,125.5,123.2,121.2,118.5,110.6,55.7$; IR (thin film): $v_{\max }\left(\mathrm{cm}^{-1}\right)=3053,2968,2928,1626,1586,1467,1256,1070,747$; 
HRMS (ESI) calcd for $\mathrm{C}_{32} \mathrm{H}_{24} \mathrm{NO}\left[\mathrm{M}+\mathrm{H}^{+}\right.$: 438.1852; Found: 438.1852; [Daicel Chiralpak ID-3 $(0.46 \mathrm{~cm} \times 15 \mathrm{~cm}), n$-hexane $/ 2$-propanol $=70 / 30, v=0.7 \mathrm{~mL} \cdot \mathrm{min}^{-1}$, $\lambda=254 \mathrm{~nm}, \mathrm{t}($ minor $)=6.89 \mathrm{~min}, \mathrm{t}($ major $)=11.25 \mathrm{~min}], 97: 3 \mathrm{er} ;[\alpha]_{\mathrm{D}}{ }^{29}=+416.6(\mathrm{c}$ $\left.=1.0, \mathrm{CHCl}_{3}\right)$.

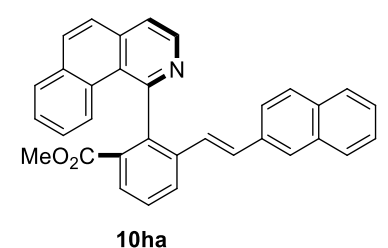

10ha. White foam, $60.4 \mathrm{mg}, 65 \%$ yield. ${ }^{1} \mathrm{H}$ NMR (400 MHz, $\left.\mathrm{CDCl}_{3}\right) \delta 8.78(\mathrm{~d}, J=$ $5.2 \mathrm{~Hz}, 1 \mathrm{H}), 8.14(\mathrm{dd}, J=8.0,1.2 \mathrm{~Hz}, 1 \mathrm{H}), 8.05(\mathrm{~d}, J=7.2 \mathrm{~Hz}, 1 \mathrm{H}), 7.91(\mathrm{~d}, J=8.8$ $\mathrm{Hz}, 1 \mathrm{H}), 7.83-7.75(\mathrm{~m}, 3 \mathrm{H}), 7.70$ (d, $J=8.8 \mathrm{~Hz}, 1 \mathrm{H}), 7.66-7.55(\mathrm{~m}, 3 \mathrm{H}), 7.51-7.40$ (m, 2H), 7.37-7.28 (m, 3H), 7.25-7.21 (m, 1H), 6.99 (d, $J=16.4 \mathrm{~Hz}, 1 \mathrm{H}), 6.90(\mathrm{dd}, J=$ 8.8, $1.6 \mathrm{~Hz}, 1 \mathrm{H}), 6.46(\mathrm{~d}, J=16.4 \mathrm{~Hz}, 1 \mathrm{H}), 3.42(\mathrm{~s}, 3 \mathrm{H}),{ }^{13} \mathrm{C} \mathrm{NMR}\left(100 \mathrm{MHz}, \mathrm{CDCl}_{3}\right)$ $\delta 166.8,157.3,143.7,143.6,137.2,136.7,134.2,133.18,133.17,132.8,132.0,131.1$, $130.4,130.1,129.9,129.4,128.9,128.3,127.9,127.8,127.4,127.1,126.7,126.68$, $126.1,125.8,125.79,125.6,125.52,125.51,123.0,121.0,51.9$; IR (thin film): $v_{\max }\left(\mathrm{cm}^{-1}\right)=3048,2948,2841,1721,1585,1507,1283,1134,746$; HRMS (ESI) calcd for $\mathrm{C}_{33} \mathrm{H}_{24} \mathrm{NO}_{2}[\mathrm{M}+\mathrm{H}]^{+}$: 466.1802; Found: 466.1800; [Daicel Chiralpak ID-3 $(0.46 \mathrm{~cm} \mathrm{x} 15 \mathrm{~cm}), n$-hexane/2-propanol $=70 / 30, v=0.7 \mathrm{~mL} \cdot \mathrm{min}^{-1}, \quad \lambda=254 \mathrm{~nm}, \mathrm{t}$ $($ minor $)=8.42 \mathrm{~min}, \mathrm{t}($ major $)=11.73 \mathrm{~min}], 97: 3 \mathrm{er} ;[\alpha]_{\mathrm{D}}^{29}=+536.7\left(\mathrm{c}=1.0, \mathrm{CHCl}_{3}\right)$.

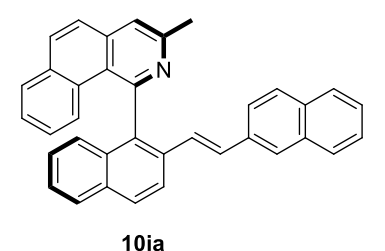

10ia. ${ }^{5} 18.3 \mathrm{mg}, 19 \%$ yield. ${ }^{1} \mathrm{H}$ NMR (400 MHz, $\left.\mathrm{CDCl}_{3}\right) \delta 8.09(\mathrm{~d}, J=8.4 \mathrm{~Hz}, 1 \mathrm{H})$, $8.04(\mathrm{~d}, J=8.8 \mathrm{~Hz}, 1 \mathrm{H}), 7.93-7.90(\mathrm{~m}, 2 \mathrm{H}), 7.79-7.73(\mathrm{~m}, 3 \mathrm{H}), 7.67-7.65(\mathrm{~m}, 2 \mathrm{H})$, 7.58-7.48 (m, 2H), 7.42-7.31 (m, 5H), $7.24(\mathrm{~d}, J=16.4 \mathrm{~Hz}, 1 \mathrm{H})$, 7.20.7.16 (m, 1H), 7.11-7.09 (m, 2H), 7.01-6.97 (m, 1H), $6.78(\mathrm{~d}, J=16.0 \mathrm{~Hz}, 1 \mathrm{H}), 2.82$ (s, 3H); [Daicel Chiralpak IE-3 $(0.46 \mathrm{~cm} \mathrm{x} 25 \mathrm{~cm}), n$-hexane $/ 2$-propanol $=80 / 20, v=0.7 \mathrm{~mL} \cdot \mathrm{min}^{-1}$, $\lambda=254 \mathrm{~nm}, \mathrm{t}($ minor $)=13.25 \mathrm{~min}, \mathrm{t}($ major $)=14.13 \mathrm{~min}], 97: 3 \mathrm{er} ;[\alpha]_{\mathrm{D}}{ }^{28}=+396.7$ $\left(\mathrm{c}=0.40, \mathrm{CHCl}_{3}\right)$. 


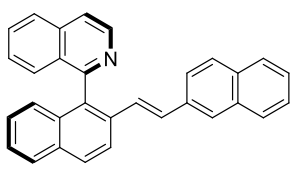

10ja

10ja. Yellow solid, m.p. $=193-195{ }^{\circ} \mathrm{C}, 64 \mathrm{mg}, 79 \%$ yield. ${ }^{1} \mathrm{H}$ NMR $(400 \mathrm{MHz}$, $\left.\mathrm{CDCl}_{3}\right) \delta 8.81(\mathrm{~d}, J=5.6 \mathrm{~Hz}, 1 \mathrm{H}), 8.07(\mathrm{~d}, J=8.8 \mathrm{~Hz}, 1 \mathrm{H}), 8.01(\mathrm{~d}, J=8.8 \mathrm{~Hz}, 1 \mathrm{H})$, $7.96(\mathrm{~d}, J=8.0 \mathrm{~Hz}, 1 \mathrm{H}), 7.90(\mathrm{~d}, J=8.0 \mathrm{~Hz}, 1 \mathrm{H}), 7.83(\mathrm{~d}, J=5.6 \mathrm{~Hz}, 1 \mathrm{H}), 7.73-7.63$ (m, 3H), 7.59-7.57 (m, 2H), 7.47-7.33 (m, 5H), 7.30-7.23 (m, 2H), $7.18(\mathrm{dd}, J=8.8$, $1.6 \mathrm{~Hz}, 1 \mathrm{H}), 7.03(\mathrm{~d}, J=8.4 \mathrm{~Hz}, 1 \mathrm{H}), 6.76(\mathrm{~d}, J=16.4 \mathrm{~Hz}, 1 \mathrm{H}) ;{ }^{13} \mathrm{C}$ NMR $(100 \mathrm{MHz}$, $\left.\mathrm{CDCl}_{3}\right) \delta 159.6,142.7,136.1,134.9,134.7,133.7,133.4,133.1,132.9,132.8,130.4$, $128.88,128.86,128.0,127.9,127.87,127.6,127.5,127.4,126.9,126.8,126.6,126.3$, 126.2, 125.8, 123.2, 122.7, 120.3; IR (thin film): $v_{\max }\left(\mathrm{cm}^{-1}\right)=3047,3019,2924,2852$, 1695, 1682, 1620, 1584, 1557, 1507, 1497, 848, 742; HRMS (ESI) calcd for $\mathrm{C}_{31} \mathrm{H}_{22} \mathrm{~N}$ $[\mathrm{M}+\mathrm{H}]^{+}:$408.1747; Found: 408.1749; [Daicel Chiralcel AD-H $(0.46 \mathrm{~cm} \times 25 \mathrm{~cm})$, $n$-hexane $/ 2$-propanol $=90 / 10, v=1.0 \mathrm{~mL} \cdot \min ^{-1}, \quad \lambda=254 \mathrm{~nm}, \mathrm{t}($ minor $)=31.21 \mathrm{~min}$, $\mathrm{t}($ major $)=44.37 \mathrm{~min}], 79: 21 \mathrm{er} ;[\alpha]_{\mathrm{D}}{ }^{27}=+254.1\left(\mathrm{c}=0.20, \mathrm{CHCl}_{3}\right)$.

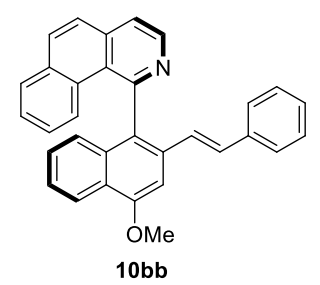

10bb. ${ }^{5} 48.7 \mathrm{mg}, 56 \%$ yield. ${ }^{1} \mathrm{H}$ NMR $\left(400 \mathrm{MHz}, \mathrm{CDCl}_{3}\right) \delta 8.89(\mathrm{~d}, J=5.2 \mathrm{~Hz}, 1 \mathrm{H})$, $8.34(\mathrm{~d}, J=8.0 \mathrm{~Hz}, 1 \mathrm{H}), 7.95(\mathrm{~d}, J=8.8 \mathrm{~Hz}, 1 \mathrm{H}), 7.86-7.77(\mathrm{~m}, 3 \mathrm{H}), 7.68(\mathrm{~d}, J=8.8$ $\mathrm{Hz}, 1 \mathrm{H}), 7.41-7.35$ (m, 2H), 7.33 (s, 1H), 7.20-7.15 (m, 1H), 7.14-6.99 (m, 8H), 6.67 $(\mathrm{d}, J=16.4 \mathrm{~Hz}, 1 \mathrm{H}), 4.20(\mathrm{~s}, 3 \mathrm{H})$; [Daicel Chiralpak ID-3 $(0.46 \mathrm{~cm} \times 15 \mathrm{~cm})$, $n$-hexane/2-propanol $=80 / 20, v=0.7 \mathrm{~mL} \cdot \min ^{-1}, \quad \lambda=254 \mathrm{~nm}, \mathrm{t}($ minor $)=15.88 \mathrm{~min}$, $\mathrm{t}($ major $)=17.45 \mathrm{~min}], 96: 4 \mathrm{er} ;[\alpha]_{\mathrm{D}}^{27}=+480.0\left(\mathrm{c}=0.20, \mathrm{CHCl}_{3}\right)$.

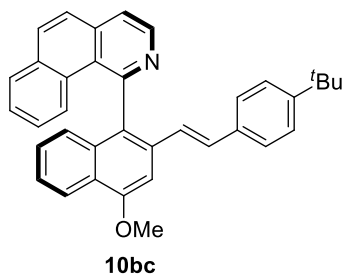


10bc. ${ }^{5} 47.6 \mathrm{mg}, 48 \%$ yield. ${ }^{1} \mathrm{H}$ NMR $\left(400 \mathrm{MHz}, \mathrm{CDCl}_{3}\right) \delta 8.89(\mathrm{~d}, J=5.2 \mathrm{~Hz}, 1 \mathrm{H})$, $8.33(\mathrm{~d}, J=8.4 \mathrm{~Hz}, 1 \mathrm{H}), 7.95(\mathrm{~d}, J=8.8 \mathrm{~Hz}, 1 \mathrm{H}), 7.88-7.76(\mathrm{~m}, 3 \mathrm{H}), 7.68(\mathrm{~d}, J=8.8$ Hz, 1H), 7.43-7.34 (m, 2H), 7.33 (s, 1H), 7.20-7.13 (m, 3H), 7.09-6.96 (m, 5H), 6.62 $(\mathrm{d}, J=16.4 \mathrm{~Hz}, 1 \mathrm{H}), 4.20$ (s, 3H), 1.20 (s, 9H); [Daicel Chiralpak ID-3 (0.46 cm x 15 $\mathrm{cm}$ ), $n$-hexane/2-propanol $=85 / 15, v=0.7 \mathrm{~mL} \cdot \mathrm{min}^{-1}, \lambda=254 \mathrm{~nm}, \mathrm{t}$ (minor) $=$ $16.58 \mathrm{~min}, \mathrm{t}($ major $)=19.12 \mathrm{~min}], 97: 3 \mathrm{er} ;[\alpha]_{\mathrm{D}}^{27}=+485.0\left(\mathrm{c}=1.00, \mathrm{CHCl}_{3}\right)$.

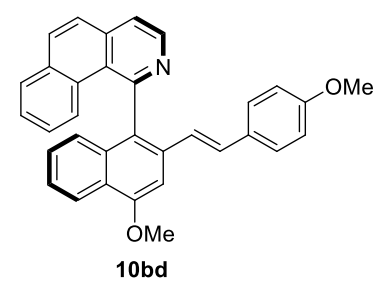

10bd. ${ }^{5} 56.8 \mathrm{mg}, 61 \%$ yield. ${ }^{1} \mathrm{H}$ NMR $\left(400 \mathrm{MHz}, \mathrm{CDCl}_{3}\right) \delta 8.89(\mathrm{~d}, J=5.2 \mathrm{~Hz}, 1 \mathrm{H})$, $8.32(\mathrm{~d}, J=8.4 \mathrm{~Hz}, 1 \mathrm{H}), 7.95(\mathrm{~d}, J=8.8 \mathrm{~Hz}, 1 \mathrm{H}), 7.86-7.76(\mathrm{~m}, 3 \mathrm{H}), 7.69(\mathrm{~d}, J=8.8$ $\mathrm{Hz}, 1 \mathrm{H}), 7.39-7.31$ (m, 2H), 7.31 (s, 1H), 7.18-7.14 (m, 1H), 7.09-6.94 (m, 5H), 6.66 $(\mathrm{d}, J=8.8 \mathrm{~Hz}, 2 \mathrm{H}), 6.53(\mathrm{~d}, J=16.0 \mathrm{~Hz}, 1 \mathrm{H}), 4.19(\mathrm{~s}, 3 \mathrm{H}), 3.69(\mathrm{~s}, 3 \mathrm{H})$; [Daicel Chiralpak ID-3 $(0.46 \mathrm{~cm} \times 15 \mathrm{~cm}), n$-hexane $/ 2$-propanol $=90 / 10, v=1.0 \mathrm{~mL} \cdot \mathrm{min}^{-1}$, $\lambda=254 \mathrm{~nm}, \mathrm{t}($ minor $)=20.13 \mathrm{~min}, \mathrm{t}($ major $)=27.83 \mathrm{~min}], 96: 4 \mathrm{er} ;[\alpha]_{\mathrm{D}}{ }^{26}=+556.9$ $\left(\mathrm{c}=1.00, \mathrm{CHCl}_{3}\right)$.

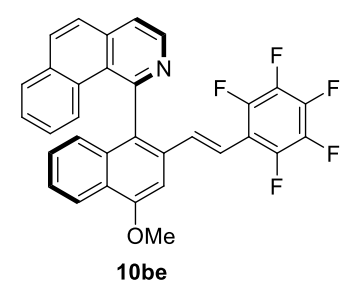

10be. ${ }^{5} 71.0 \mathrm{mg}, 67 \%$ yield. ${ }^{1} \mathrm{H}$ NMR $\left(400 \mathrm{MHz}, \mathrm{CDCl}_{3}\right) \delta 8.89(\mathrm{~d}, J=5.2 \mathrm{~Hz}, 1 \mathrm{H})$, $8.37(\mathrm{~d}, J=8.0 \mathrm{~Hz}, 1 \mathrm{H}), 7.97(\mathrm{~d}, J=8.8 \mathrm{~Hz}, 1 \mathrm{H}), 7.89-7.79(\mathrm{~m}, 3 \mathrm{H}), 7.57(\mathrm{~d}, J=8.8$ $\mathrm{Hz}, 1 \mathrm{H}), 7.50-7.35$ (m, 2H), 7.25-7.19 (m, 2H), 7.12 (d, J=8.0 Hz, 1H), 7.09-7.04 (m, 1H), $6.95(\mathrm{~d}, J=16.8 \mathrm{~Hz}, 1 \mathrm{H}), 6.85(\mathrm{~d}, J=16.8 \mathrm{~Hz}, 1 \mathrm{H}), 4.22(\mathrm{~s}, 3 \mathrm{H}) ;{ }^{19} \mathrm{~F}$ NMR $(376$ $\left.\mathrm{Hz}, \mathrm{CDCl}_{3}\right) \delta-143.0(\mathrm{~m}),-156.7(\mathrm{~m}),-163.2(\mathrm{~m})$; [Daicel Chiralpak ID-3 (0.46 cm x $15 \mathrm{~cm}$ ), $n$-hexane/2-propanol $=70 / 30, v=0.7 \mathrm{~mL} \cdot \mathrm{min}^{-1}, \quad \lambda=254 \mathrm{~nm}, \mathrm{t}$ (minor) $=$ 5.75 min, $\mathrm{t}$ (major) $=7.72 \mathrm{~min}], 94: 6 \mathrm{er} ;[\alpha]_{\mathrm{D}}{ }^{27}=+376.3\left(\mathrm{c}=1.00, \mathrm{CHCl}_{3}\right)$. 


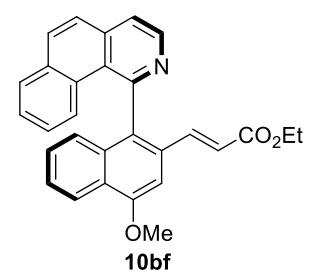

10bf. ${ }^{5} 83.4 \mathrm{mg}, 96 \%$ yield. ${ }^{1} \mathrm{H}$ NMR $\left(400 \mathrm{MHz}, \mathrm{CDCl}_{3}\right) \delta 8.86(\mathrm{~d}, J=5.2 \mathrm{~Hz}, 1 \mathrm{H})$, $8.35(\mathrm{~d}, J=8.4 \mathrm{~Hz}, 1 \mathrm{H}), 7.94(\mathrm{~d}, J=8.8 \mathrm{~Hz}, 1 \mathrm{H}), 7.88-7.71(\mathrm{~m}, 3 \mathrm{H}), 7.51-7.37$ (m, 3H), 7.29-7.17 (m, 3H), 7.05-7.01 (m, 2H), $6.39(\mathrm{~d}, J=15.6 \mathrm{~Hz}, 1 \mathrm{H}), 4.14(\mathrm{~s}, 3 \mathrm{H})$, $4.02(\mathrm{q}, J=7.2 \mathrm{~Hz}, 2 \mathrm{H}), 1.13(\mathrm{t}, J=7.2 \mathrm{~Hz}, 3 \mathrm{H})$; [Daicel Chiralpak ID-3 (0.46 cm x $15 \mathrm{~cm}$ ), $n$-hexane/2-propanol $=80 / 20, v=0.7 \mathrm{~mL} \cdot \min ^{-1}, \quad \lambda=254 \mathrm{~nm}, \mathrm{t}$ (major) $=$ $17.85 \mathrm{~min}, \mathrm{t}($ minor $)=21.75 \mathrm{~min}], 97: 3 \mathrm{er} ;[\alpha]_{\mathrm{D}}^{22}=+292.6\left(\mathrm{c}=1.00, \mathrm{CHCl}_{3}\right)$.

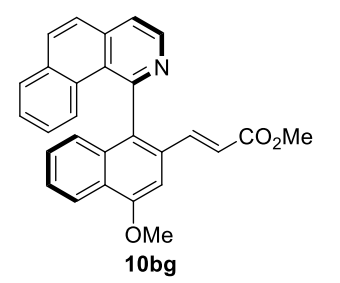

10bg. ${ }^{5} 76.7 \mathrm{mg}, 91 \%$ yield. ${ }^{1} \mathrm{H} \mathrm{NMR}\left(400 \mathrm{MHz}, \mathrm{CDCl}_{3}\right) \delta 8.86(\mathrm{~d}, J=5.2 \mathrm{~Hz}, 1 \mathrm{H})$, $8.34(\mathrm{~d}, J=8.4 \mathrm{~Hz}, 1 \mathrm{H}), 7.96(\mathrm{~d}, J=8.8 \mathrm{~Hz}, 1 \mathrm{H}), 7.86-7.80(\mathrm{~m}, 3 \mathrm{H}), 7.50-7.37$ (m, 3H), $7.29(\mathrm{~d}, J=15.6 \mathrm{~Hz}, 1 \mathrm{H}), 7.22-7.14(\mathrm{~m}, 2 \mathrm{H}), 7.05-6.99(\mathrm{~m}, 2 \mathrm{H}), 6.39(\mathrm{~d}, J=$ $15.6 \mathrm{~Hz}, 1 \mathrm{H}), 4.15$ (s, 3H), 3.56 (s, 3H); [Daicel Chiralpak ID-3 (0.46 cm x $15 \mathrm{~cm})$, $n$-hexane/2-propanol $=70 / 30, v=0.7 \mathrm{~mL} \cdot \min ^{-1}, \quad \lambda=254 \mathrm{~nm}, \mathrm{t}$ (major) $=12.51 \mathrm{~min}$, $\mathrm{t}($ minor $)=14.69 \mathrm{~min}], 96: 4 \mathrm{er} ;[\alpha]_{\mathrm{D}}^{27}=+290.6\left(\mathrm{c}=1.00, \mathrm{CHCl}_{3}\right)$.

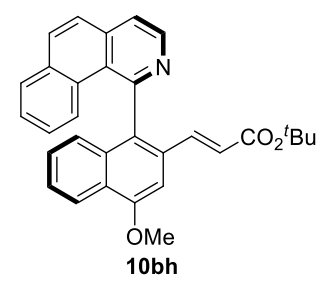

10bh. ${ }^{5} 64.2 \mathrm{mg}, 70 \%$ yield. ${ }^{1} \mathrm{H}$ NMR $\left(400 \mathrm{MHz}, \mathrm{CDCl}_{3}\right) \delta 8.87(\mathrm{~d}, J=5.2 \mathrm{~Hz}, 1 \mathrm{H})$, $8.34(\mathrm{~d}, J=8.4 \mathrm{~Hz}, 1 \mathrm{H}), 7.95(\mathrm{~d}, J=8.8 \mathrm{~Hz}, 1 \mathrm{H}), 7.88-7.76(\mathrm{~m}, 3 \mathrm{H}), 7.53-7.34(\mathrm{~m}$, $3 \mathrm{H}), 7.25-7.15(\mathrm{~m}, 3 \mathrm{H}), 7.08-6.98(\mathrm{~m}, 2 \mathrm{H}), 6.32(\mathrm{~d}, J=15.6 \mathrm{~Hz}, 1 \mathrm{H}), 4.15(\mathrm{~s}, 3 \mathrm{H})$, 1.32 (s, 9H); [Daicel Chiralpak ID-3 (0.46 cm x $15 \mathrm{~cm}), n$-hexane/2-propanol = 80/20, 
$v=0.7 \mathrm{~mL} \cdot \min ^{-1}, \quad \lambda=254 \mathrm{~nm}, \mathrm{t}$ (major) $=9.29 \mathrm{~min}, \mathrm{t}($ minor $\left.)=11.71 \mathrm{~min}\right], 97: 3 \mathrm{er}$; $[\alpha]_{\mathrm{D}}^{29}=+301.5\left(\mathrm{c}=1.00, \mathrm{CHCl}_{3}\right)$.

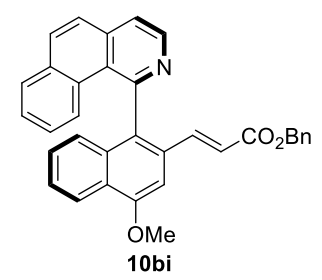

10bi. ${ }^{5} 57.6 \mathrm{mg}, 58 \%$ yield. ${ }^{1} \mathrm{H}$ NMR $\left(400 \mathrm{MHz}, \mathrm{CDCl}_{3}\right) \delta 8.86(\mathrm{~d}, J=5.2 \mathrm{~Hz}, 1 \mathrm{H})$, $8.35(\mathrm{~d}, J=8.4 \mathrm{~Hz}, 1 \mathrm{H}), 7.95(\mathrm{~d}, J=8.8 \mathrm{~Hz}, 1 \mathrm{H}), 7.89-7.76(\mathrm{~m}, 3 \mathrm{H}), 7.51-7.36(\mathrm{~m}$, $3 \mathrm{H}), 7.33-7.28(\mathrm{~m}, 4 \mathrm{H}), 7.25-7.12(\mathrm{~m}, 4 \mathrm{H}), 7.05-7.01(\mathrm{~m}, 2 \mathrm{H}), 6.44(\mathrm{~d}, J=15.6 \mathrm{~Hz}$, 1H), $5.00(\mathrm{~s}, 2 \mathrm{H}), 4.14(\mathrm{~s}, 3 \mathrm{H})$; [Daicel Chiralpak ID-3 $(0.46 \mathrm{~cm} \times 15 \mathrm{~cm})$, $n$-hexane/2-propanol $=65 / 25, v=0.7 \mathrm{~mL} \cdot \min ^{-1}, \quad \lambda=254 \mathrm{~nm}, \mathrm{t}$ (major) $=13.55 \mathrm{~min}$, $\mathrm{t}($ minor $)=16.07 \mathrm{~min}], 96: 4 \mathrm{er} ;[\alpha]_{\mathrm{D}}^{32}=+292.6\left(\mathrm{c}=1.00, \mathrm{CHCl}_{3}\right)$.

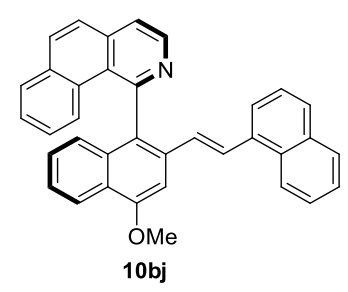

10bj. ${ }^{5} 66.0 \mathrm{mg}, 68 \%$ yield. ${ }^{1} \mathrm{H}$ NMR $\left(400 \mathrm{MHz}, \mathrm{CDCl}_{3}\right) \delta 8.88(\mathrm{~d}, J=4.8 \mathrm{~Hz}, 1 \mathrm{H})$, $8.37(\mathrm{~d}, J=8.4 \mathrm{~Hz}, 1 \mathrm{H}), 7.95(\mathrm{~d}, J=8.8 \mathrm{~Hz}, 1 \mathrm{H}), 7.87-7.64(\mathrm{~m}, 7 \mathrm{H}), 7.61(\mathrm{~d}, J=8.4$ $\mathrm{Hz}, 1 \mathrm{H}), 7.46-7.30(\mathrm{~m}, 5 \mathrm{H}), 7.24-7.17(\mathrm{~m}, 2 \mathrm{H}), 7.15-7.01(\mathrm{~m}, 3 \mathrm{H}), 6.69(\mathrm{~d}, J=16.0$ $\mathrm{Hz}, \quad 1 \mathrm{H}), 4.25$ (s, 3H); [Daicel Chiralpak ID-3 $(0.46 \mathrm{~cm} \quad \mathrm{x} \quad 15 \mathrm{~cm})$, $n$-hexane/2-propanol $=70 / 30, v=0.7 \mathrm{~mL} \cdot \min ^{-1}, \quad \lambda=254 \mathrm{~nm}, \mathrm{t}($ minor $)=8.59 \mathrm{~min}$, $\mathrm{t}($ major $)=11.46 \mathrm{~min}], 95: 5 \mathrm{er} ;[\alpha]_{\mathrm{D}}^{27}=+414.1\left(\mathrm{c}=1.00, \mathrm{CHCl}_{3}\right)$.

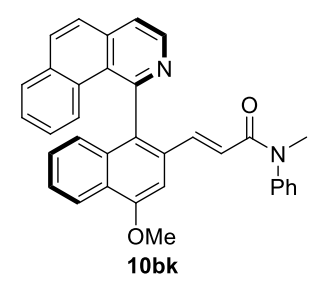

10bk. ${ }^{5} 72.0 \mathrm{mg}, 73 \%$ yield. ${ }^{1} \mathrm{H} \mathrm{NMR}\left(400 \mathrm{MHz}, \mathrm{CDCl}_{3}\right) \delta 8.79(\mathrm{~d}, J=5.2 \mathrm{~Hz}, 1 \mathrm{H})$, $8.27(\mathrm{~d}, J=7.6 \mathrm{~Hz}, 1 \mathrm{H}), 7.96(\mathrm{~d}, J=8.8 \mathrm{~Hz}, 1 \mathrm{H}), 7.86-7.76(\mathrm{~m}, 3 \mathrm{H}), 7.47(\mathrm{~d}, J=8.4$ $\mathrm{Hz}, 1 \mathrm{H})$, 7.42-7.37 (m, 2H), 7.34-7.24 (m, 4H), 7.17-7.13 (m, 1H), 7.03-6.92 (m, 4H), 
$6.81(\mathrm{~s}, 1 \mathrm{H}), 6.13(\mathrm{~d}, J=15.6 \mathrm{~Hz}, 1 \mathrm{H}), 3.98(\mathrm{~s}, 3 \mathrm{H}), 3.21$ (s, 3H); [Daicel Chiralpak ID-3 $(0.46 \mathrm{~cm} \times 15 \mathrm{~cm}), n$-hexane $/ 2$-propanol $=50 / 50, v=0.7 \mathrm{~mL} \cdot \mathrm{min}^{-1}, \lambda=254 \mathrm{~nm}$, $\mathrm{t}($ major $)=13.61 \mathrm{~min}, \mathrm{t}($ minor $)=21.42 \mathrm{~min}], 97: 3 \mathrm{er} ;[\alpha]_{\mathrm{D}}^{28}=+207.1(\mathrm{c}=1.00$, $\left.\mathrm{CHCl}_{3}\right)$.

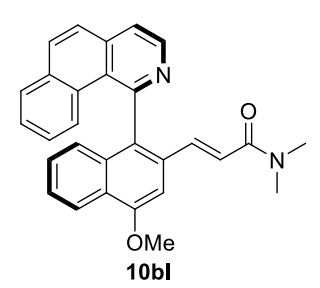

10bl. ${ }^{5} 48.5 \mathrm{mg}, 56 \%$ yield. ${ }^{1} \mathrm{H}$ NMR (400 MHz, $\left.\mathrm{CDCl}_{3}\right) \delta 8.84(\mathrm{~d}, J=5.2 \mathrm{~Hz}, 1 \mathrm{H})$, $8.34(\mathrm{~d}, J=8.4 \mathrm{~Hz}, 1 \mathrm{H}), 7.94(\mathrm{~d}, J=8.8 \mathrm{~Hz}, 1 \mathrm{H}), 7.86-7.73(\mathrm{~m}, 3 \mathrm{H}), 7.54(\mathrm{~d}, J=8.8$ $\mathrm{Hz}, 1 \mathrm{H}), 7.45-7.37$ (m, 2H), 7.22-7.13 (m, 3H), 7.05-7.01 (m, 2H), 6.58 (d, J= 15.6 Hz, 1H), 4.16 (s, 3H), 2.82 (s, 3H), 2.80 (s, 3H); [Daicel Chiralpak ID-3 (0.46 cm x $15 \mathrm{~cm}$ ), $n$-hexane $/ 2$-propanol $=50 / 50, v=0.7 \mathrm{~mL} \cdot \min -1, \lambda=254 \mathrm{~nm}, \mathrm{t}$ (major) $=$ $20.52 \mathrm{~min}, \mathrm{t}($ minor $)=33.07 \mathrm{~min}], 98: 2 \mathrm{er} ;[\alpha]_{\mathrm{D}}{ }^{28}=+273.5\left(\mathrm{c}=1.00, \mathrm{CHCl}_{3}\right)$.

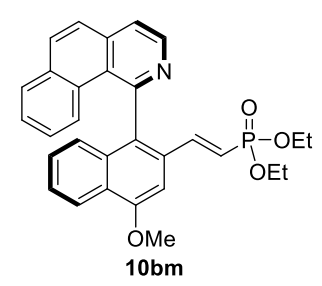

10bm. ${ }^{5} 83.2 \mathrm{mg}, 84 \%$ yield. ${ }^{1} \mathrm{H}$ NMR $\left(400 \mathrm{MHz}, \mathrm{CDCl}_{3}\right) \delta 8.86(\mathrm{~d}, J=5.2 \mathrm{~Hz}, 1 \mathrm{H})$, $8.37(\mathrm{~d}, J=8.0 \mathrm{~Hz}, 1 \mathrm{H}), 7.96(\mathrm{~d}, J=8.8 \mathrm{~Hz}, 1 \mathrm{H}), 7.86-7.80(\mathrm{~m}, 3 \mathrm{H}), 7.52-7.37(\mathrm{~m}$, $3 \mathrm{H}), 7.29-7.22(\mathrm{~m}, 1 \mathrm{H}), 7.19-7.11(\mathrm{~m}, 2 \mathrm{H}), 7.07-7.02(\mathrm{~m}, 1 \mathrm{H}), 6.90(\mathrm{dd}, J=22.4$, $17.6 \mathrm{~Hz}, 1 \mathrm{H}), 6.17-6.04(\mathrm{~m}, 1 \mathrm{H}), 4.16(\mathrm{~s}, 3 \mathrm{H}), 3.76-3.47(\mathrm{~m}, 4 \mathrm{H}), 1.04(\mathrm{t}, J=7.2 \mathrm{~Hz}$, $3 \mathrm{H}), 0.95(\mathrm{t}, J=7.2 \mathrm{~Hz}, 3 \mathrm{H})$; [Daicel Chiralpak ID-3 $(0.46 \mathrm{~cm} \times 15 \mathrm{~cm})$, $n$-hexane/2-propanol $=50 / 50, v=0.7 \mathrm{~mL} \cdot \min ^{-1}, \lambda=254 \mathrm{~nm}, \mathrm{t}$ (major) $=7.56 \mathrm{~min}, \mathrm{t}$ $($ minor $)=11.83 \mathrm{~min}], 93: 7 \mathrm{er} ;[\alpha]_{\mathrm{D}}{ }^{31}=+261.7\left(\mathrm{c}=0.20, \mathrm{CHCl}_{3}\right)$.

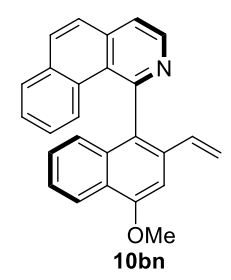


10bn. ${ }^{5} 26.6 \mathrm{mg}, 37 \%$ yield. ${ }^{1} \mathrm{H}$ NMR $\left(400 \mathrm{MHz}, \mathrm{CDCl}_{3}\right) \delta 8.87(\mathrm{~d}, J=5.2 \mathrm{~Hz}, 1 \mathrm{H})$, $8.33(\mathrm{~d}, J=8.4 \mathrm{~Hz}, 1 \mathrm{H}), 7.96(\mathrm{~d}, J=8.4 \mathrm{~Hz}, 1 \mathrm{H}), 7.83-7.80(\mathrm{~m}, 3 \mathrm{H}), 7.62(\mathrm{~d}, J=8.8$ $\mathrm{Hz}, 1 \mathrm{H}), 7.43-7.38$ (m, 2H), 7.23-7.14 (m, 2H), 7.11-6.95 (m, 2H), 6.26 (dd, J= 17.2, $11.2 \mathrm{~Hz}, 1 \mathrm{H}), 5.69(\mathrm{~d}, J=17.2 \mathrm{~Hz}, 1 \mathrm{H}), 5.00(\mathrm{~d}, J=11.2 \mathrm{~Hz}, 1 \mathrm{H}), 4.16(\mathrm{~s}, 3 \mathrm{H})$; [Daicel Chiralpak IC $(0.46 \mathrm{~cm} \times 25 \mathrm{~cm}), n$-hexane/2-propanol $=60 / 40, v=0.7$ $\mathrm{mL} \cdot \min ^{-1}, \lambda=254 \mathrm{~nm}, \mathrm{t}$ (major) $=11.40 \mathrm{~min}, \mathrm{t}($ minor $\left.)=20.44 \mathrm{~min}\right], 96: 4 \mathrm{er} ;[\alpha]_{\mathrm{D}}{ }^{29}=$ $+199.0\left(\mathrm{c}=0.20, \mathrm{CHCl}_{3}\right)$. 


\section{References:}

1. Zhu, S.-F.; Yang, Y.; Wang, L.-X.; Liu, B.; Zhou, Q.-L. Org. Lett. 2005, 7, 2333.

2. Mei, T.-S.; Giri, R.; Maugel, N.; Yu, J.-Q. Angew. Chem. Int. Ed. 2008, 47, 5215.

3. Wolter, M.; Nordmann, G.; Job, G. E.; Buchwald, S. L. Org. Lett. 2002, 4, 973.

4. Ye, B.; Cramer, N. J. Am. Chem. Soc. 2013, 135, 636.

5. Zheng, J.; You, S.-L. Angew. Chem. Int. Ed. 2014, 53, 13244. 
X-Ray crystal structures of $(S)$-K3a and $(S)$-K3d

(S)-K3d (CCDC 1455371)

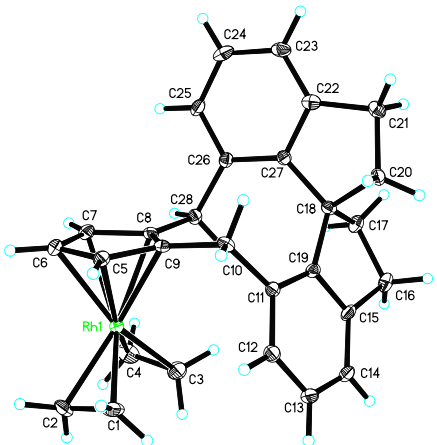

Table 1. Crystal data and structure refinement for mo_dm15424_0m.

Identification code

Empirical formula

Formula weight

Temperature

Wavelength

Crystal system

Space group

Unit cell dimensions

Volume

Z

Density (calculated)

Absorption coefficient

$\mathrm{F}(000)$

Crystal size

Theta range for data collection

Index ranges

Reflections collected

Independent reflections

Completeness to theta $=26.000^{\circ}$

Absorption correction

Max. and min. transmission

Refinement method

Data / restraints / parameters

Goodness-of-fit on $\mathrm{F}^{2}$ mo_dm15424_0m

C28 H29 Rh

468.42

$130 \mathrm{~K}$

$0.71073 \AA$

Orthorhombic

P 212121

$\mathrm{a}=10.2511(14) \AA \quad \alpha=90^{\circ}$.

$\mathrm{b}=12.0614(17) \AA \quad \beta=90^{\circ}$.

$\mathrm{c}=16.840(2) \AA \quad \gamma=90^{\circ}$.

2082.2(5) $\AA^{3}$

4

$1.494 \mathrm{Mg} / \mathrm{m}^{3}$

$0.832 \mathrm{~mm}^{-1}$

968

$0.25 \times 0.2 \times 0.12 \mathrm{~mm}^{3}$

2.077 to $30.535^{\circ}$.

$-13<=\mathrm{h}<=14,-13<=\mathrm{k}<=17,-23<=\mathrm{l}<=23$

20779

$6329[\mathrm{R}(\mathrm{int})=0.0748]$

$100.0 \%$

Semi-empirical from equivalents

0.7461 and 0.6078

Full-matrix least-squares on $\mathrm{F}^{2}$

6329 / 0 / 262

1.025 
Final R indices [I>2sigma(I)]

$\mathrm{R}$ indices (all data)

Absolute structure parameter

Extinction coefficient

Largest diff. peak and hole

$$
\begin{aligned}
& \mathrm{R} 1=0.0452, \mathrm{wR} 2=0.0877 \\
& \mathrm{R} 1=0.0666, \mathrm{wR} 2=0.0960 \\
& -0.01(3) \\
& \text { n/a }
\end{aligned}
$$

0.613 and -1.137 e. $\AA^{-3}$

(S)-K3a (CCDC 1455372)

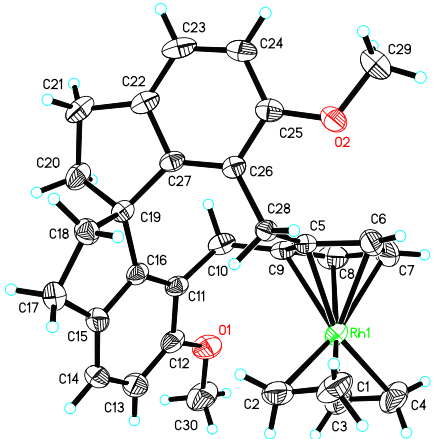

Table 1. Crystal data and structure refinement for mo_dm15783_0m.

Identification code

Empirical formula

Formula weight

Temperature

Wavelength

Crystal system

Space group

Unit cell dimensions

Volume

Z

Density (calculated)

Absorption coefficient

$\mathrm{F}(000)$

Crystal size

Theta range for data collection

Index ranges

Reflections collected

Independent reflections mo_dm15783_0m

C30 H33 O2 Rh

528.47

$296.15 \mathrm{~K}$

$0.71073 \AA$

Orthorhombic

P 212121

$$
\begin{array}{ll}
\mathrm{a}=7.156(3) \AA & \alpha=90^{\circ} . \\
\mathrm{b}=14.439(6) \AA & \beta=90^{\circ} . \\
\mathrm{c}=23.483(9) \AA & \gamma=90^{\circ} .
\end{array}
$$

2426.5(16) $\AA^{3}$

4

$1.447 \mathrm{Mg} / \mathrm{m}^{3}$

$0.729 \mathrm{~mm}^{-1}$

1096

$0.2 \times 0.12 \times 0.1 \mathrm{~mm}^{3}$

1.656 to $30.906^{\circ}$.

$-10<=\mathrm{h}<=9,-20<=\mathrm{k}<=20,-30<=\mathrm{l}<=33$

24822

$7588[\mathrm{R}($ int $)=0.0630]$ 
Completeness to theta $=26.000^{\circ}$

Absorption correction

Max. and min. transmission

Refinement method

Data / restraints / parameters

Goodness-of-fit on $\mathrm{F}^{2}$

Final R indices [I $>2 \operatorname{sigma}(\mathrm{I})]$

$\mathrm{R}$ indices (all data)

Absolute structure parameter

Extinction coefficient

Largest diff. peak and hole
$100.0 \%$

Semi-empirical from equivalents

0.7461 and 0.6810

Full-matrix least-squares on $\mathrm{F}^{2}$

7588 / 0 / 300

0.982

$\mathrm{R} 1=0.0463, \mathrm{wR} 2=0.0838$

$\mathrm{R} 1=0.0999, \mathrm{wR} 2=0.0996$

$-0.01(2)$

$\mathrm{n} / \mathrm{a}$

0.357 and -0.438 e. $\AA^{-3}$ 
Copies of NMR spectra and HPLC chromatographs

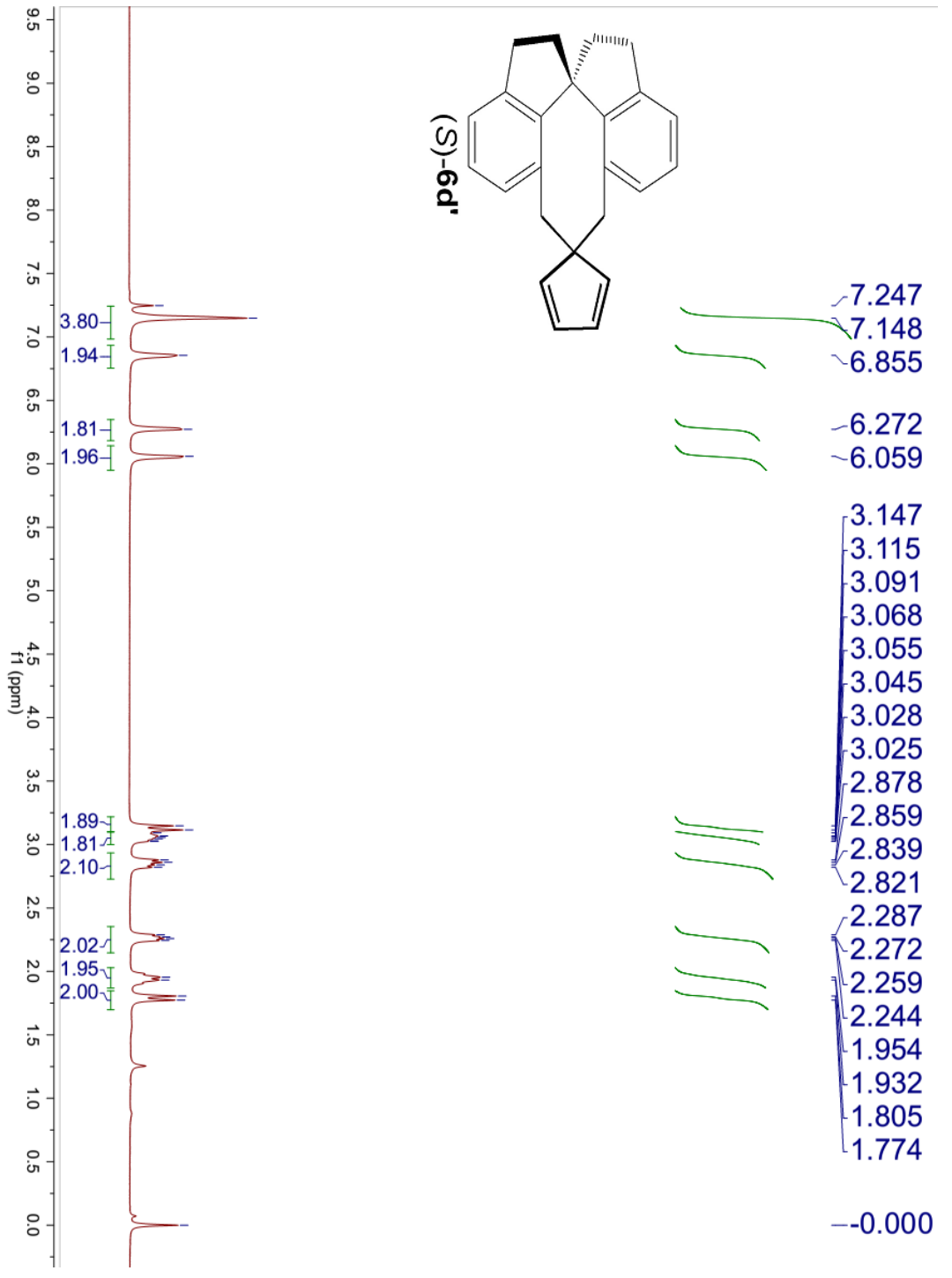



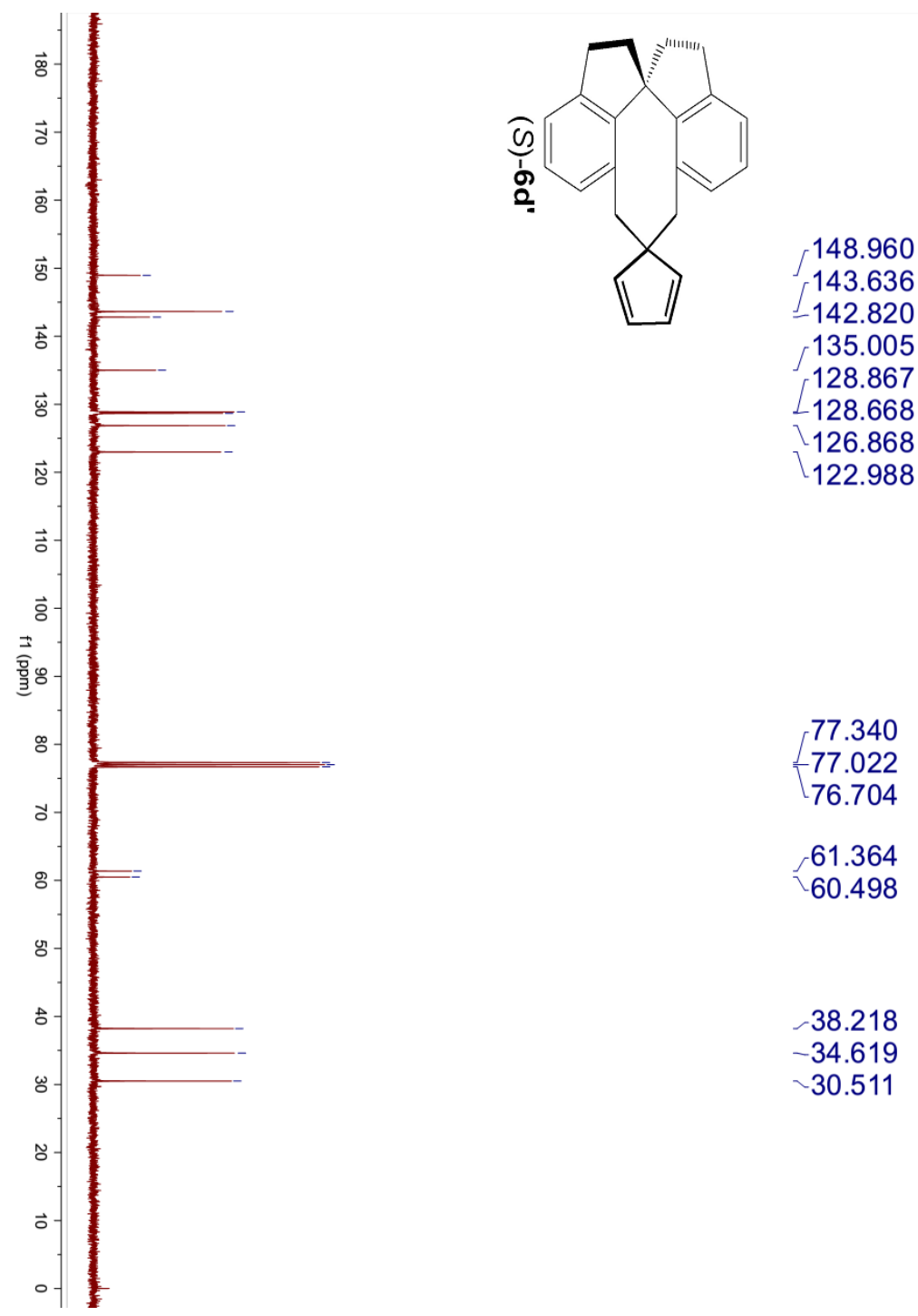

77.340

$-77.022$

76.704

61.364

60.498

38.218

$-34.619$

30.511 


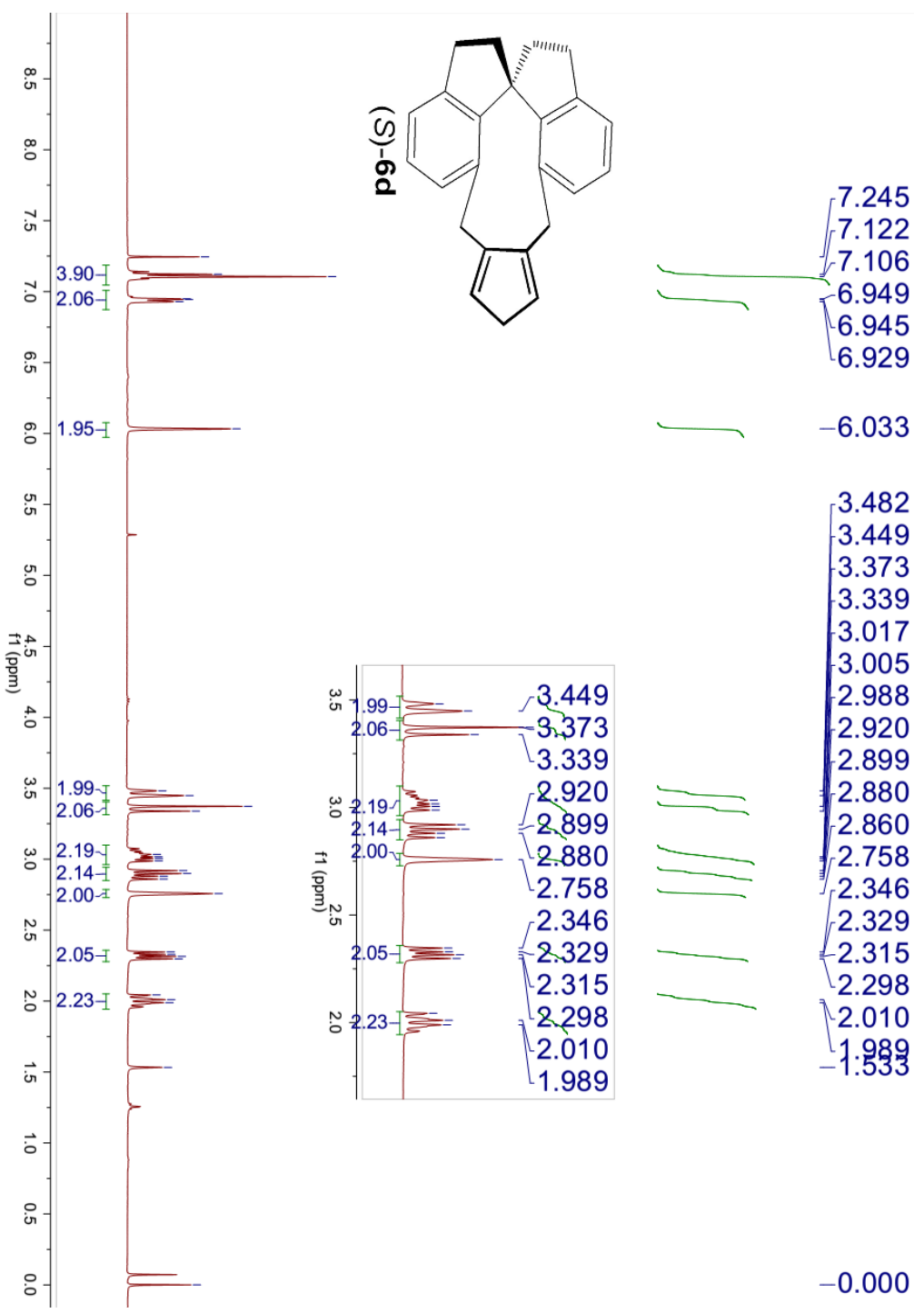




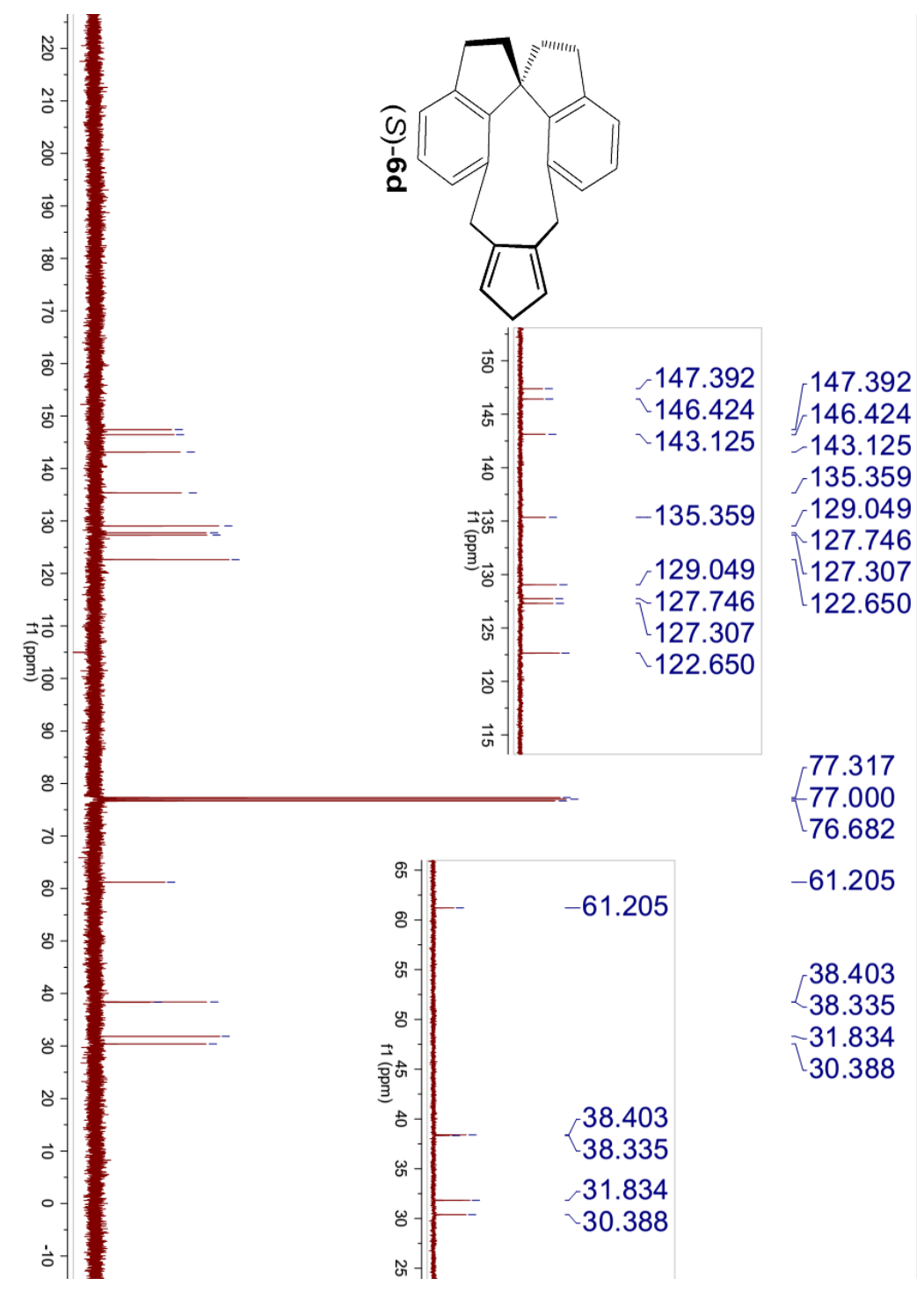



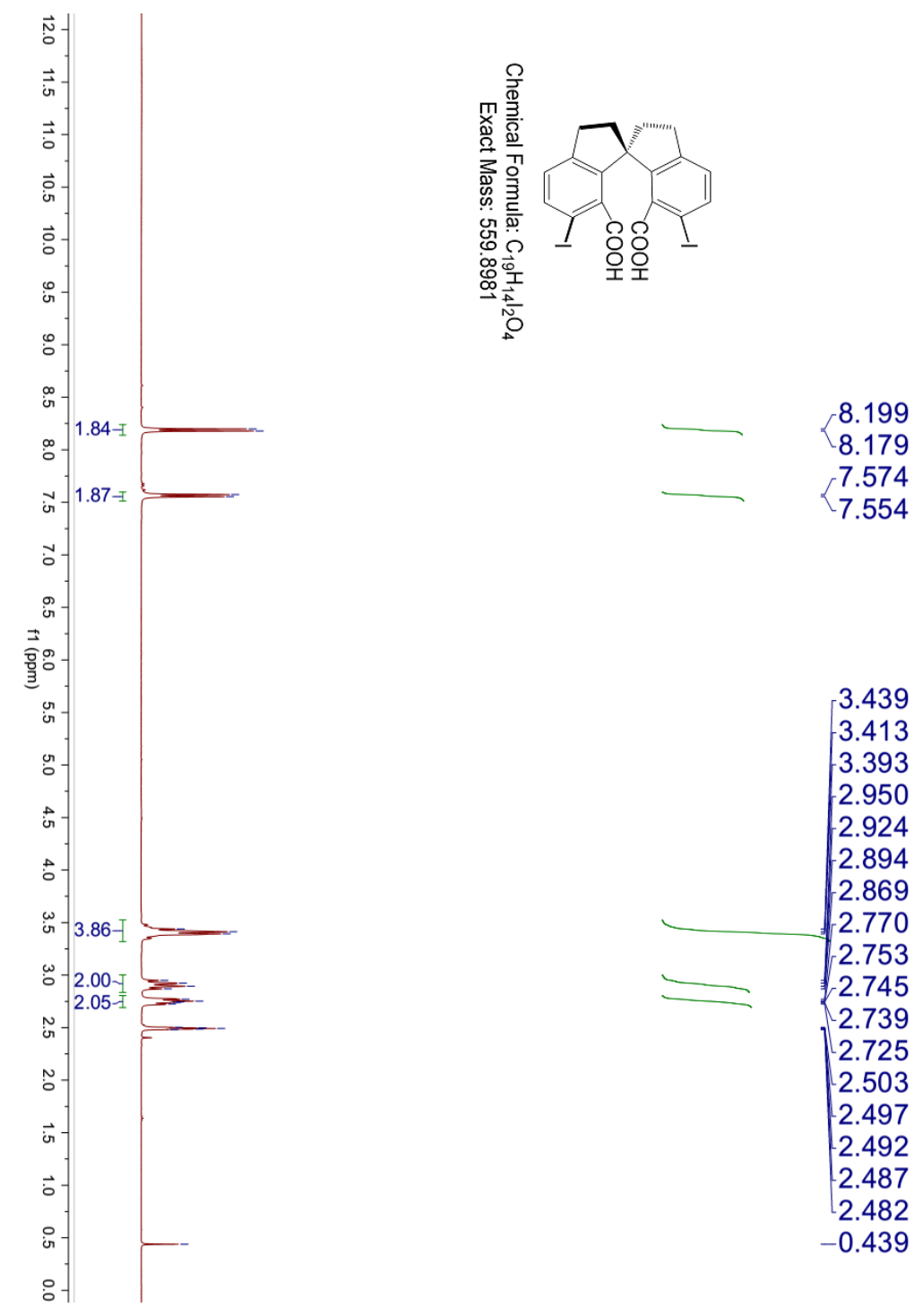


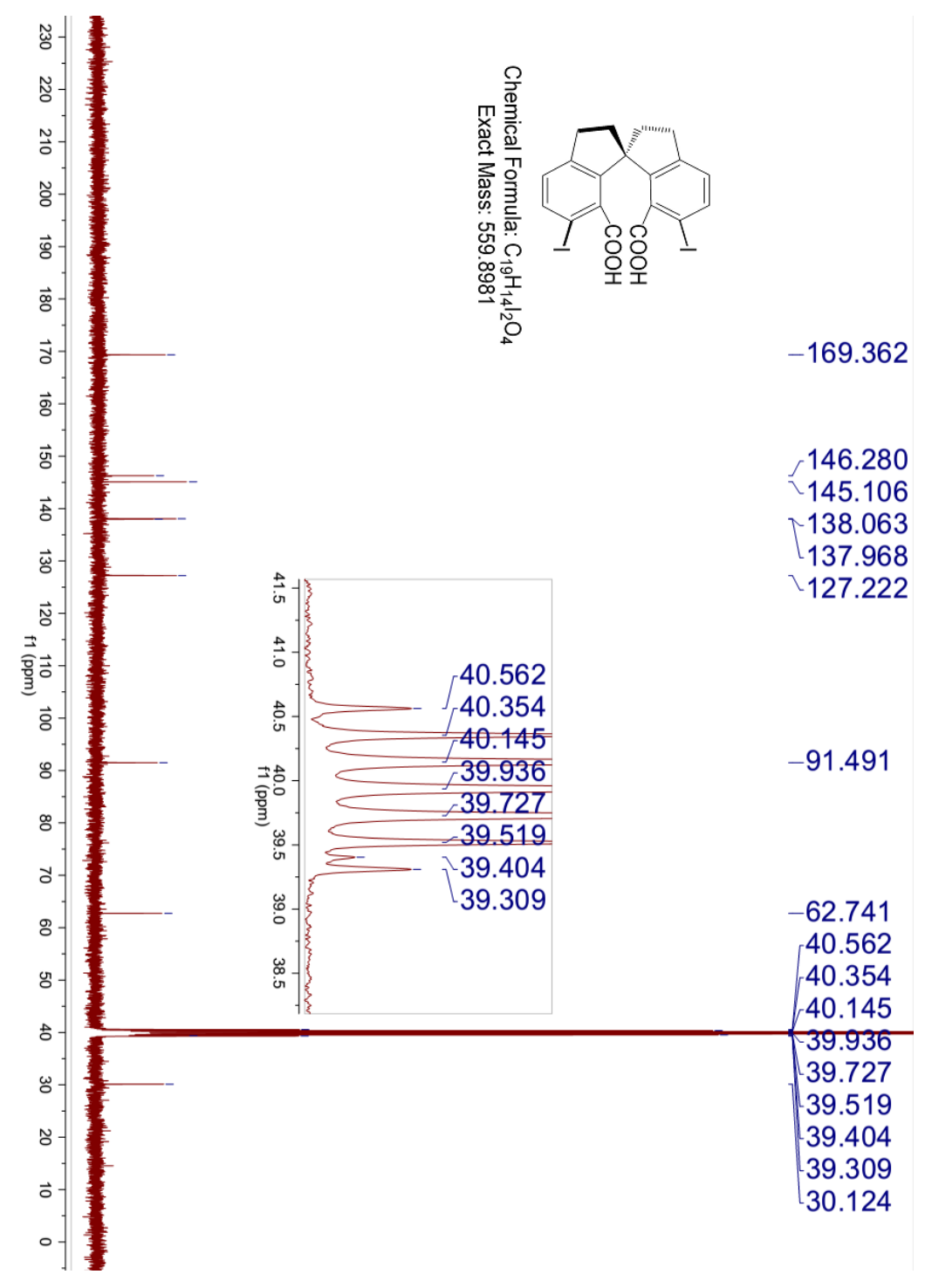




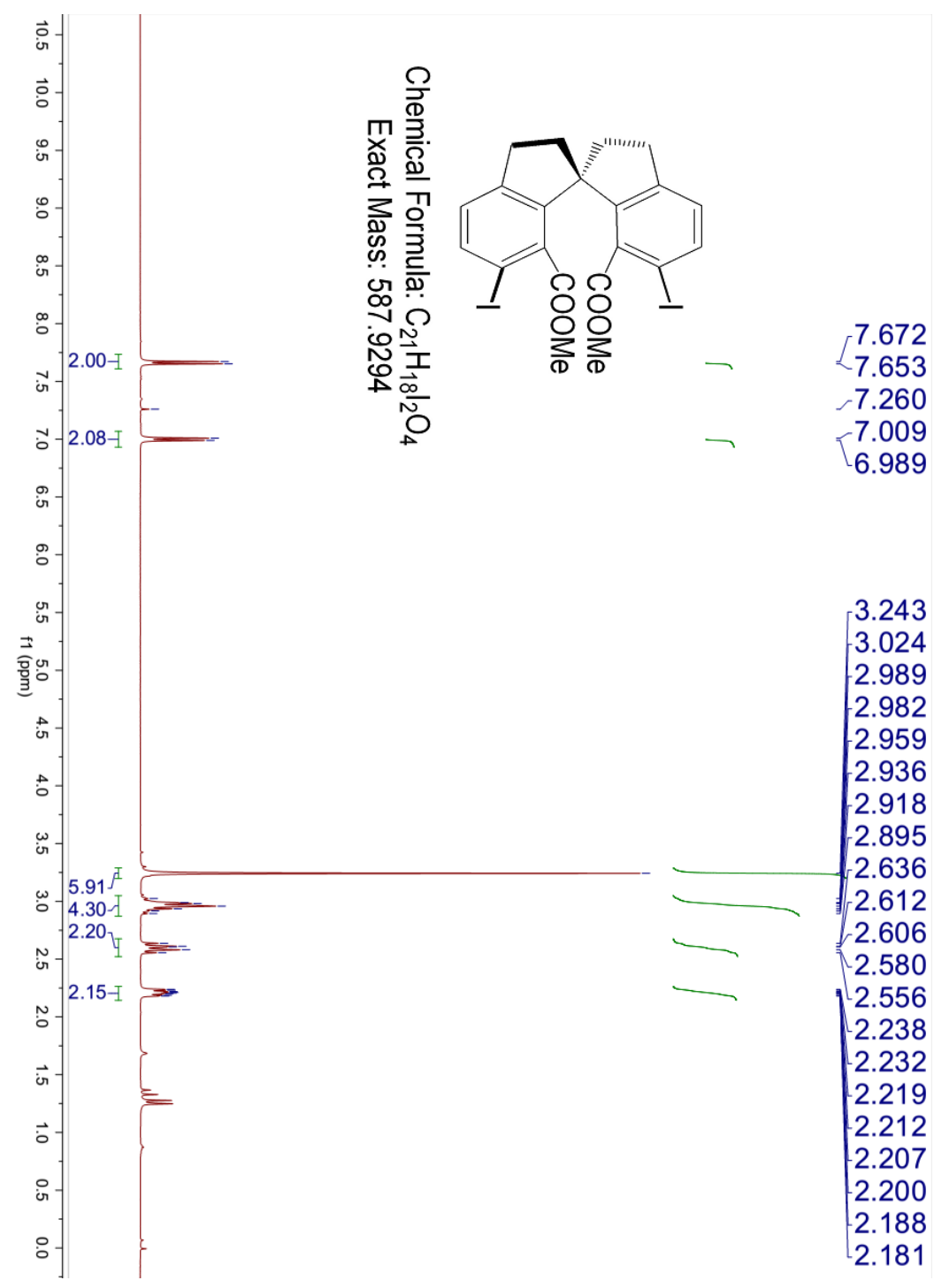



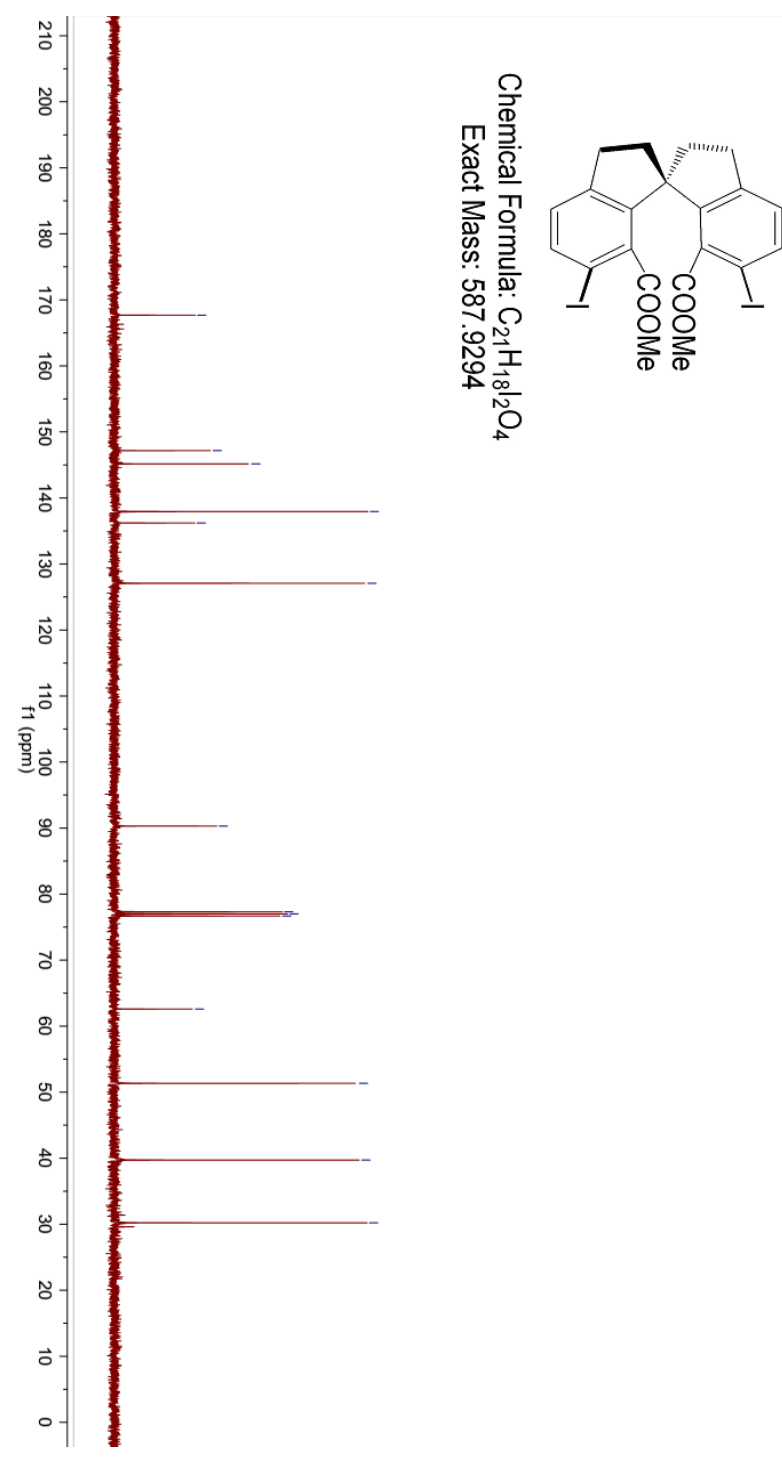

$-167.687$

147.173

$\backslash 145.157$

$-137.938$

$-136.214$

$-127.080$

$-90.310$

77.318

77.000

76.681

$-62.599$

$-51.341$

$-39.718$

$-30.215$ 

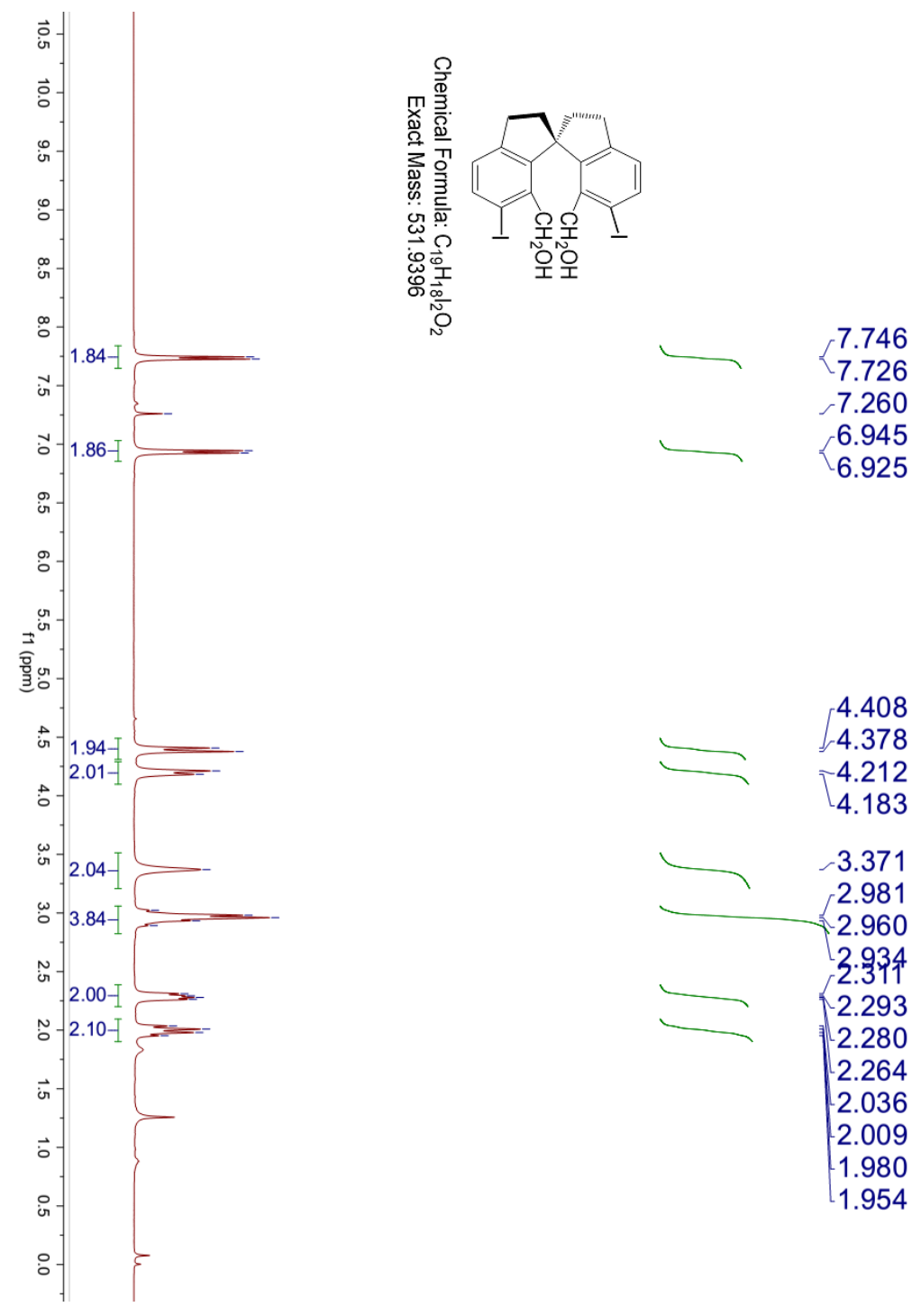

2.981

2.960

3.934

$\underbrace{2.334}_{2.293}$

2.280

2.264

2.036

2.009

1.980

1.954 

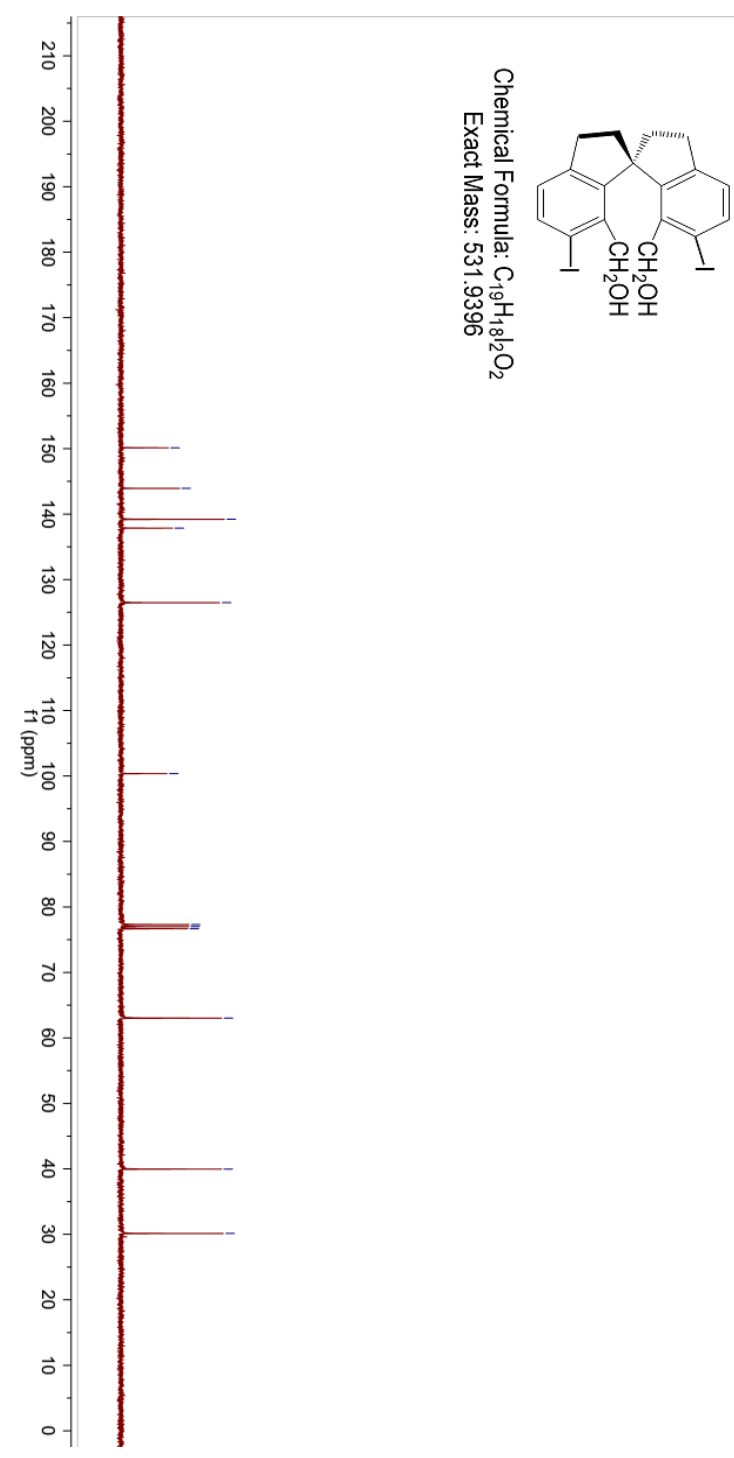

150.136

143.929

139.210

137.859

$-126.472$

$-100.366$

77.318

$-77.000$

76.682

$-63.021$

$-39.964$

$-30.134$ 


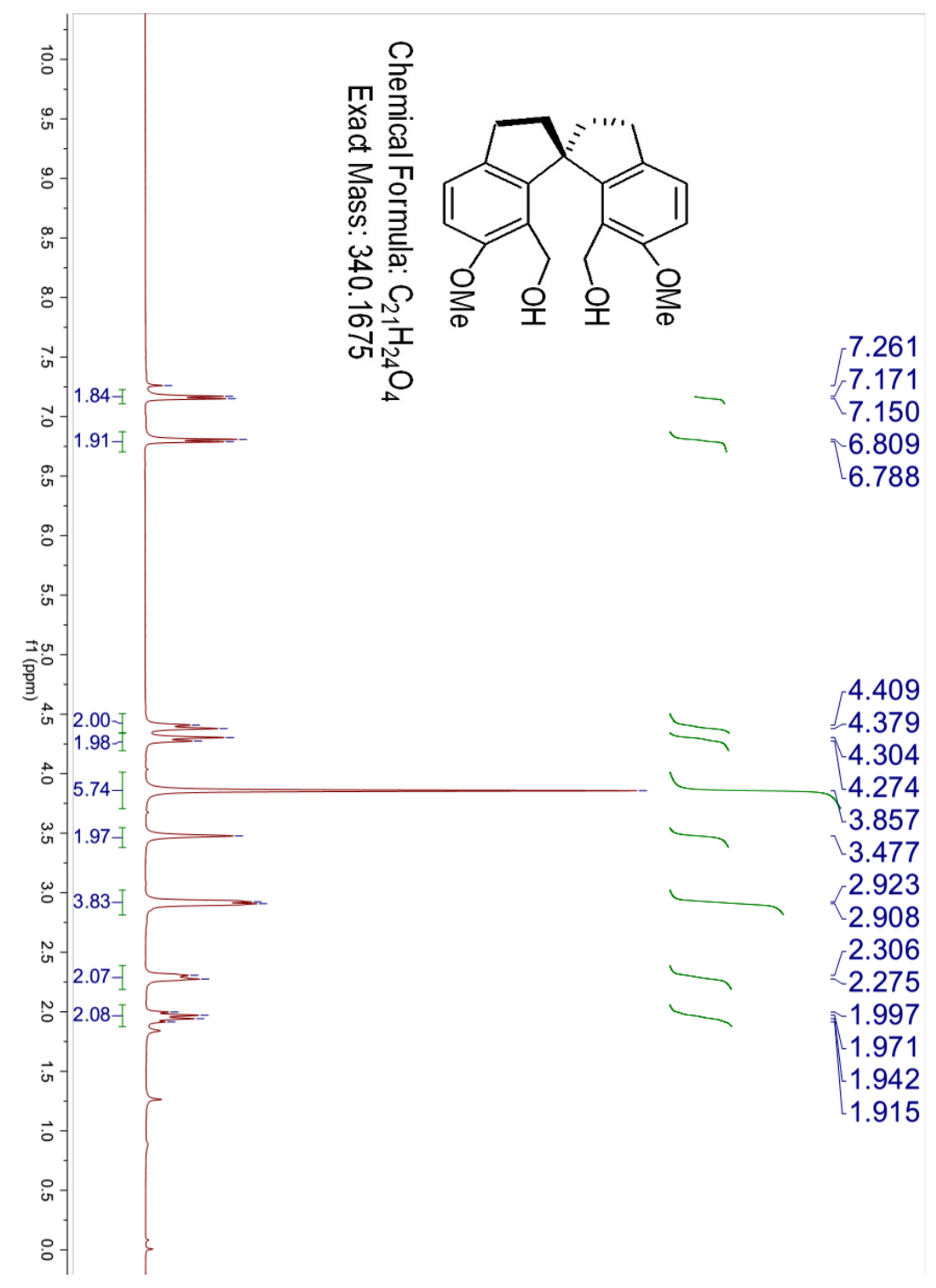




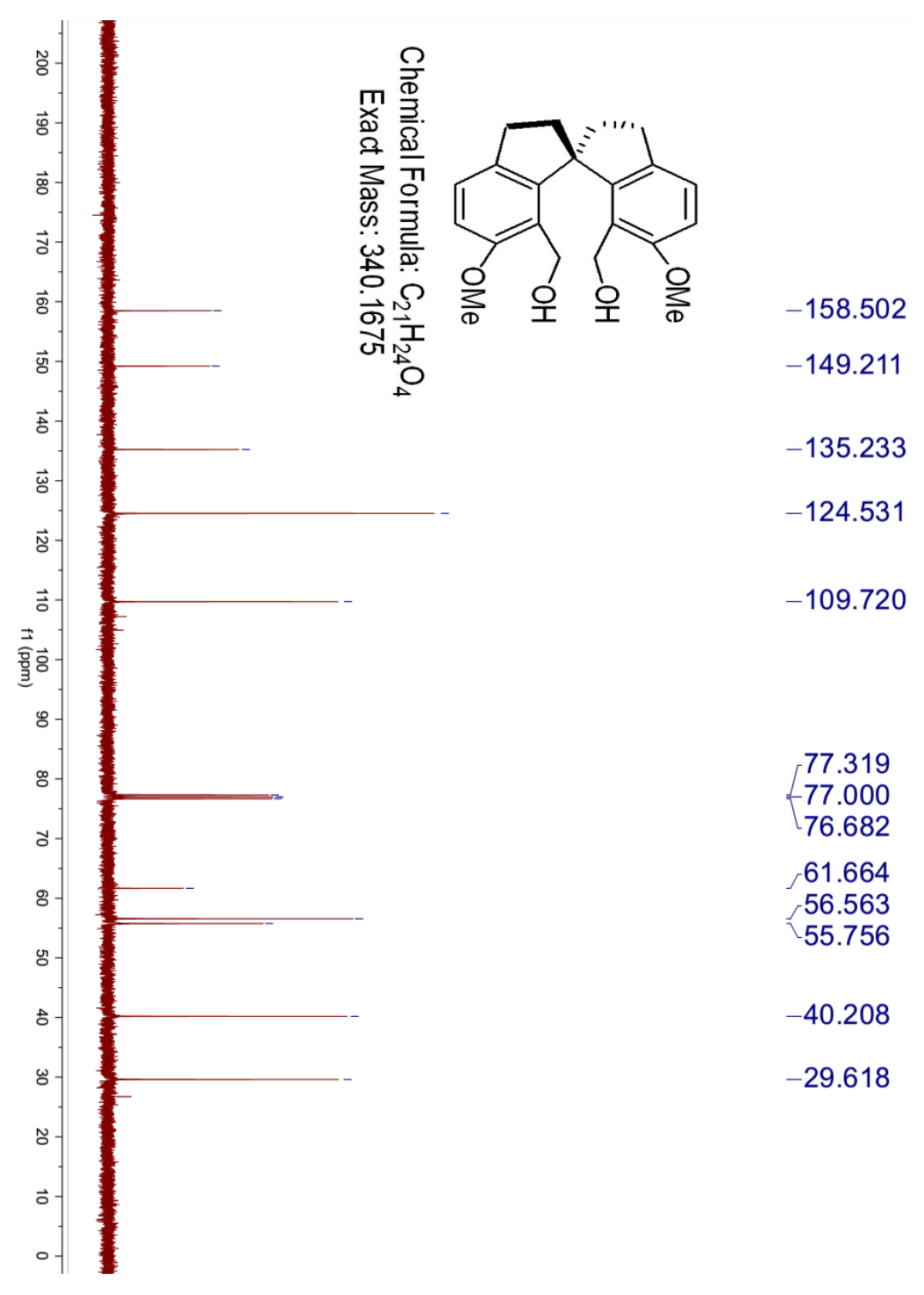



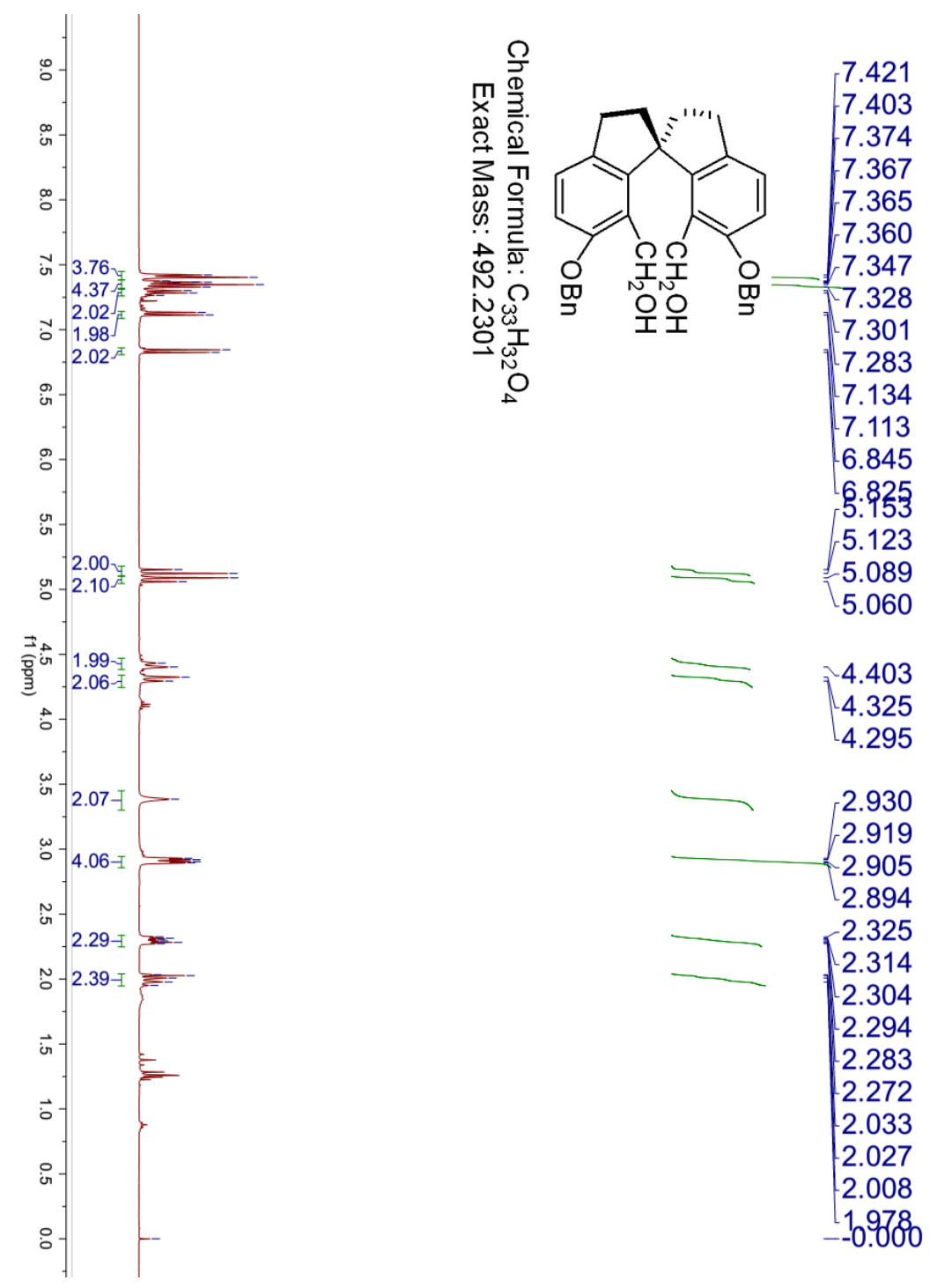

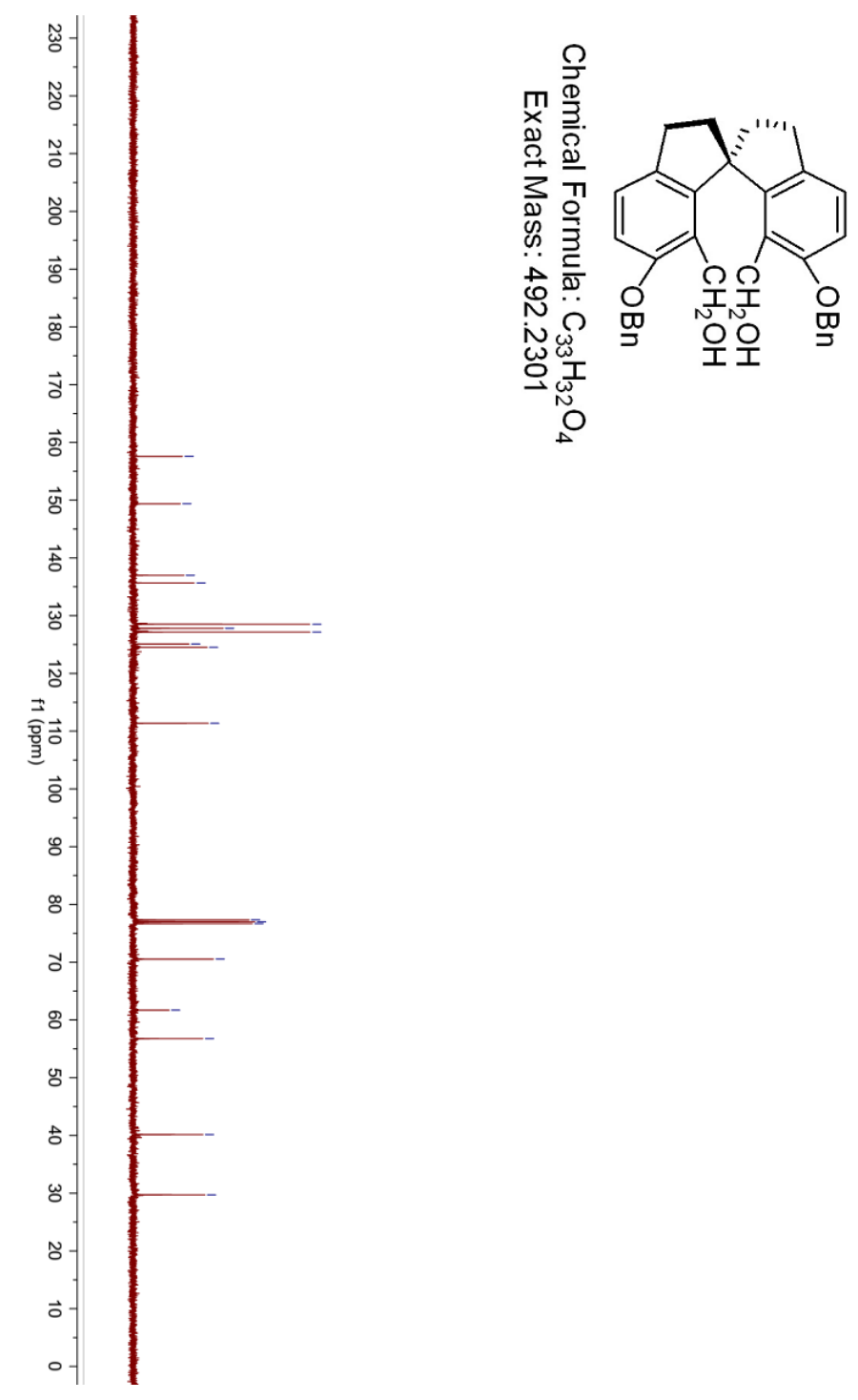

$-157.587$

$-149.375$

135.668

128.532

127.809

127.155

$-124.526$

77.318

77.000

76.681

70.537

61.702

56.777

$-40.155$

$-29.728$ 

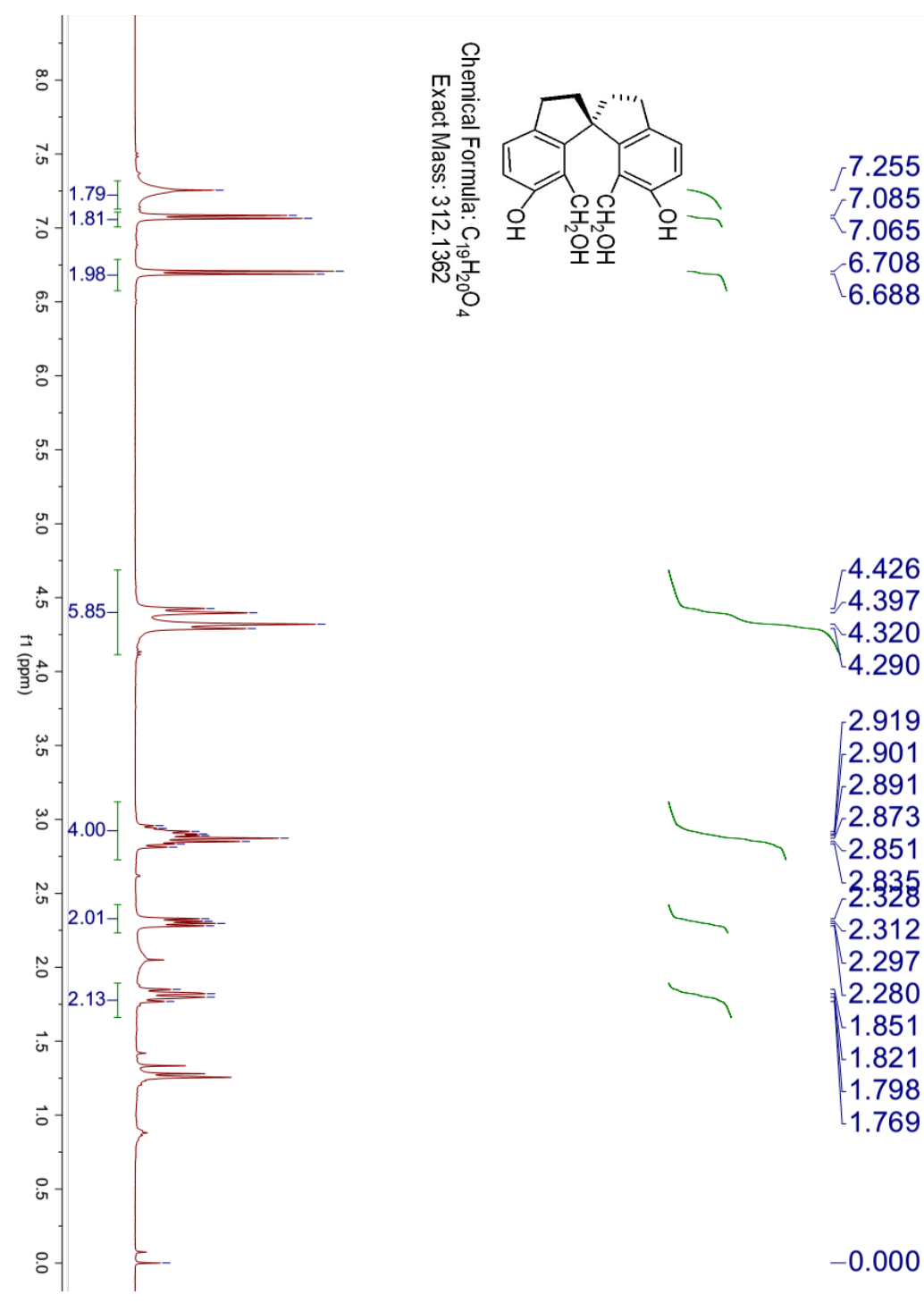

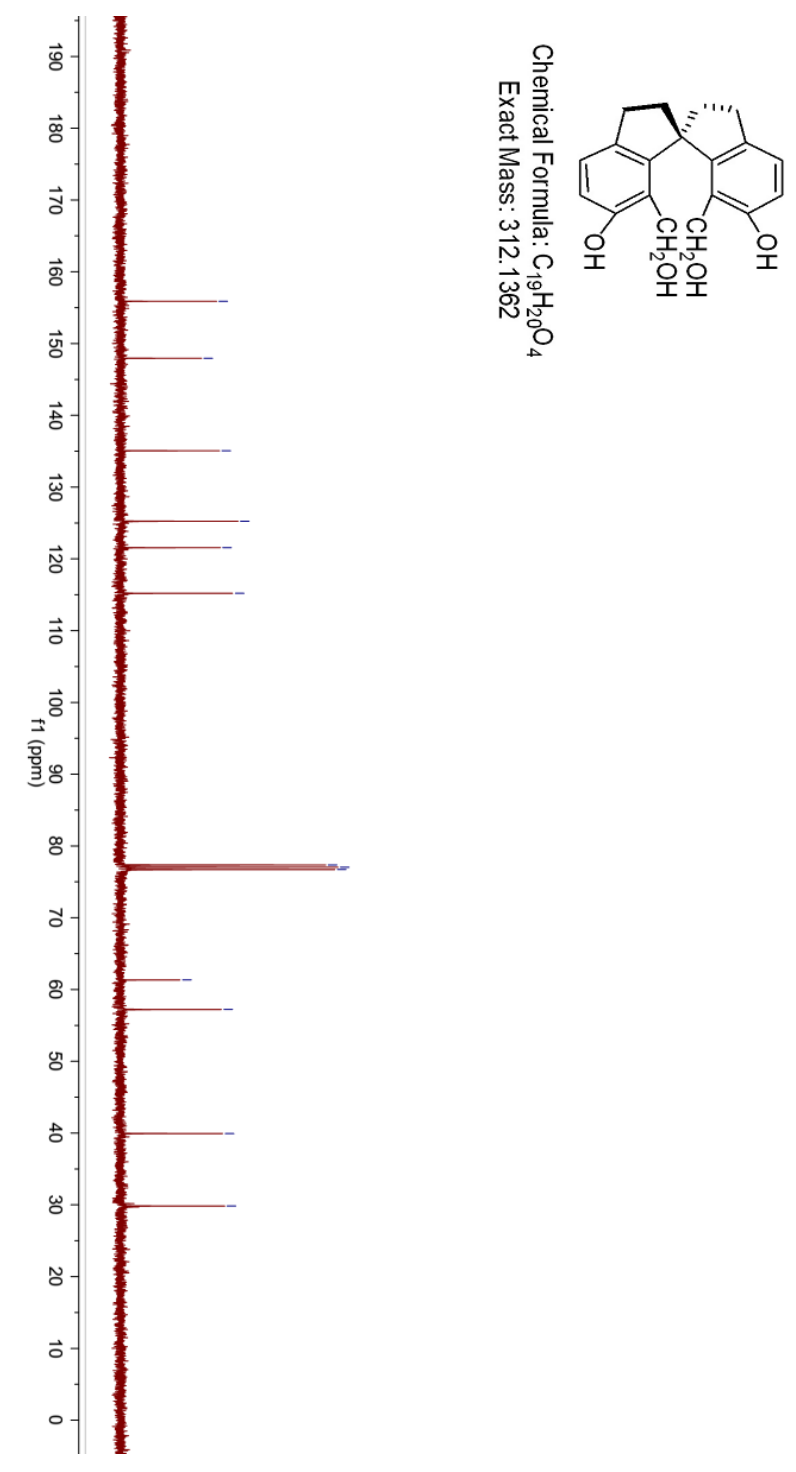

-155.896
-147.955

135.069

125.250

$-121.561$

115.205

77.353

$-77.035$

76.718

$-61.330$

$-57.219$

$-39.935$

$-29.837$ 


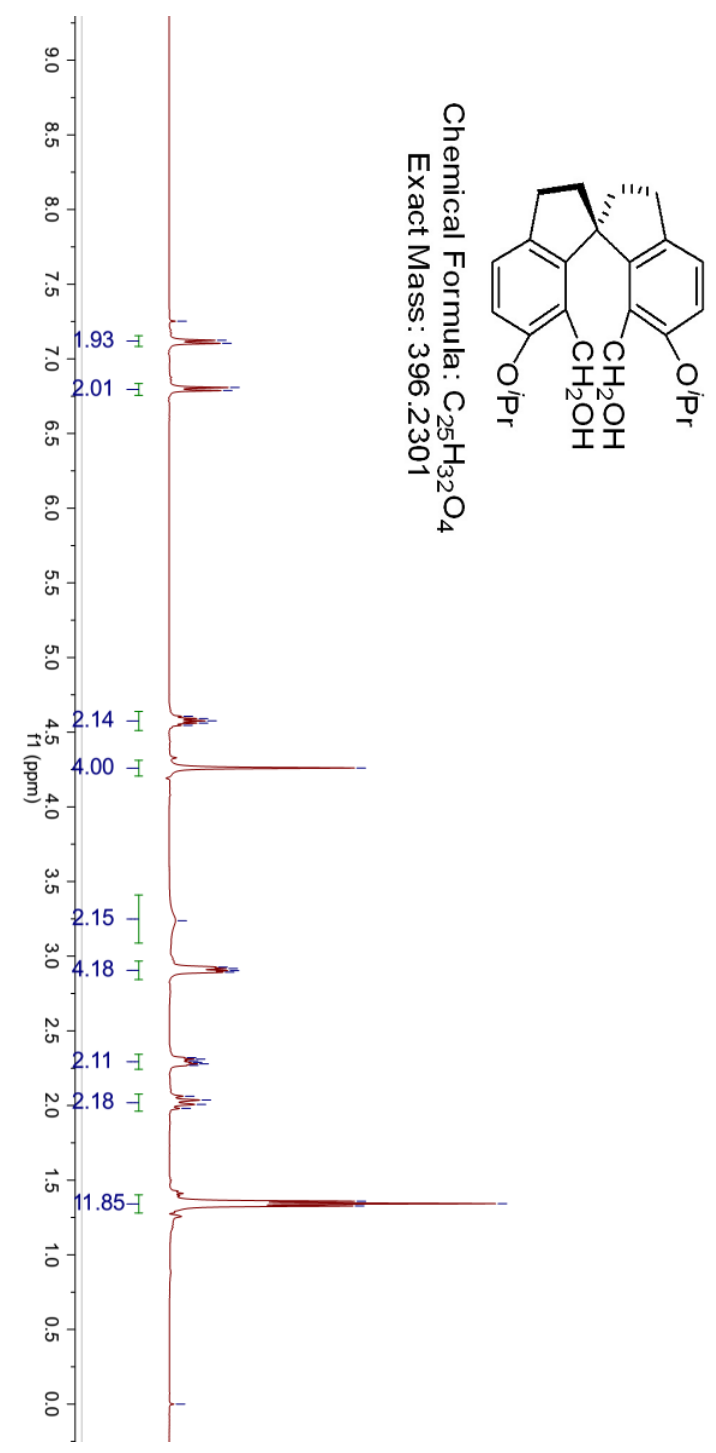




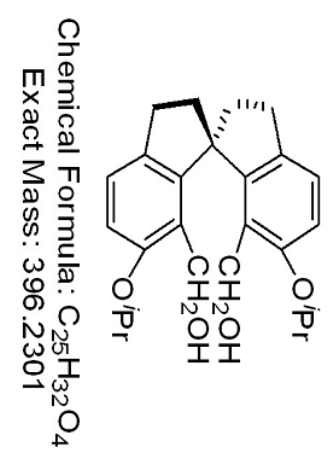

$\backslash 135.316$

$-126.010$

124.359

112.597

77.375

$-77.057$

76.739

70.991

61.789

57.324

39.972

29.825

ऽ2.289 


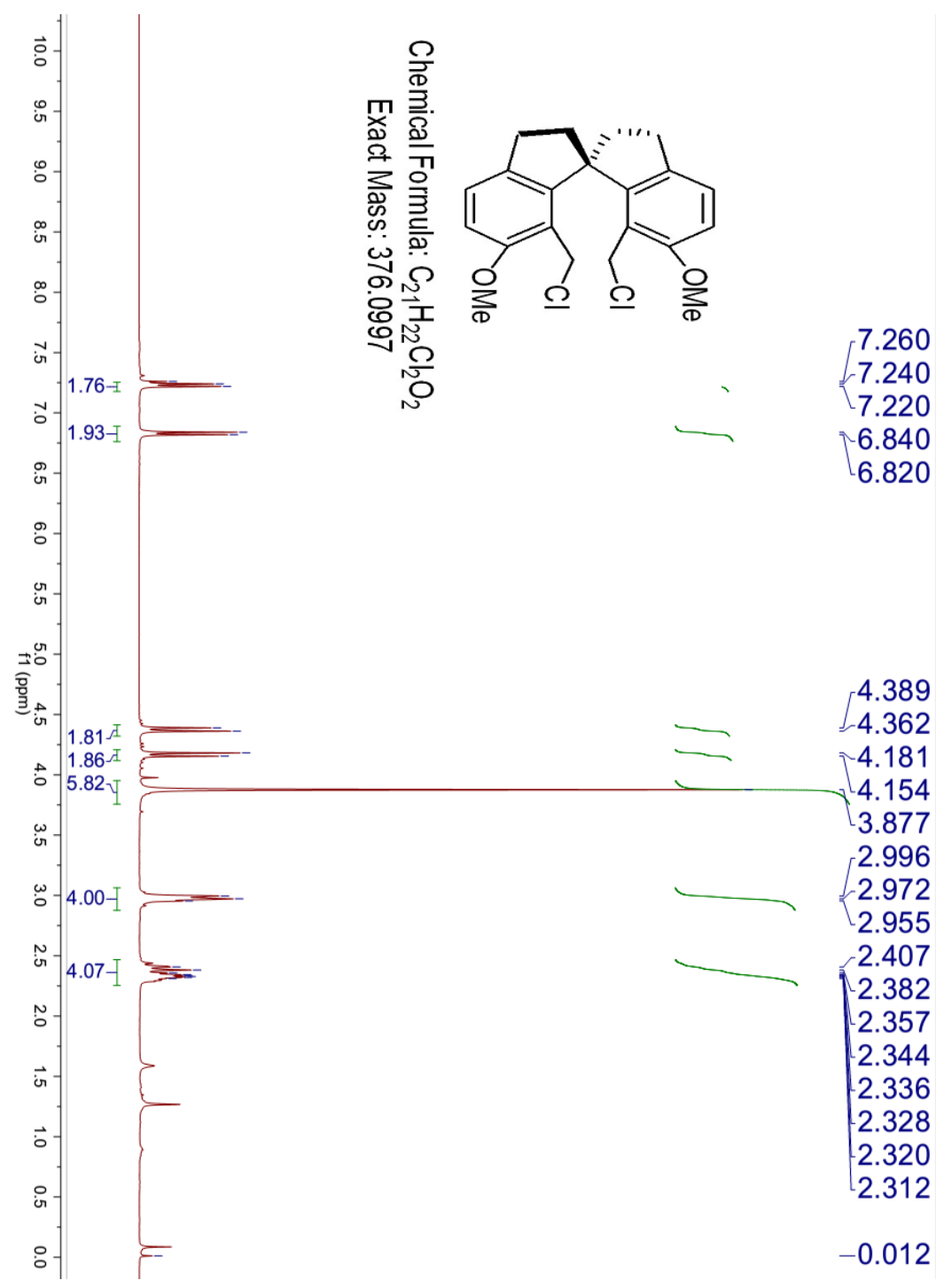



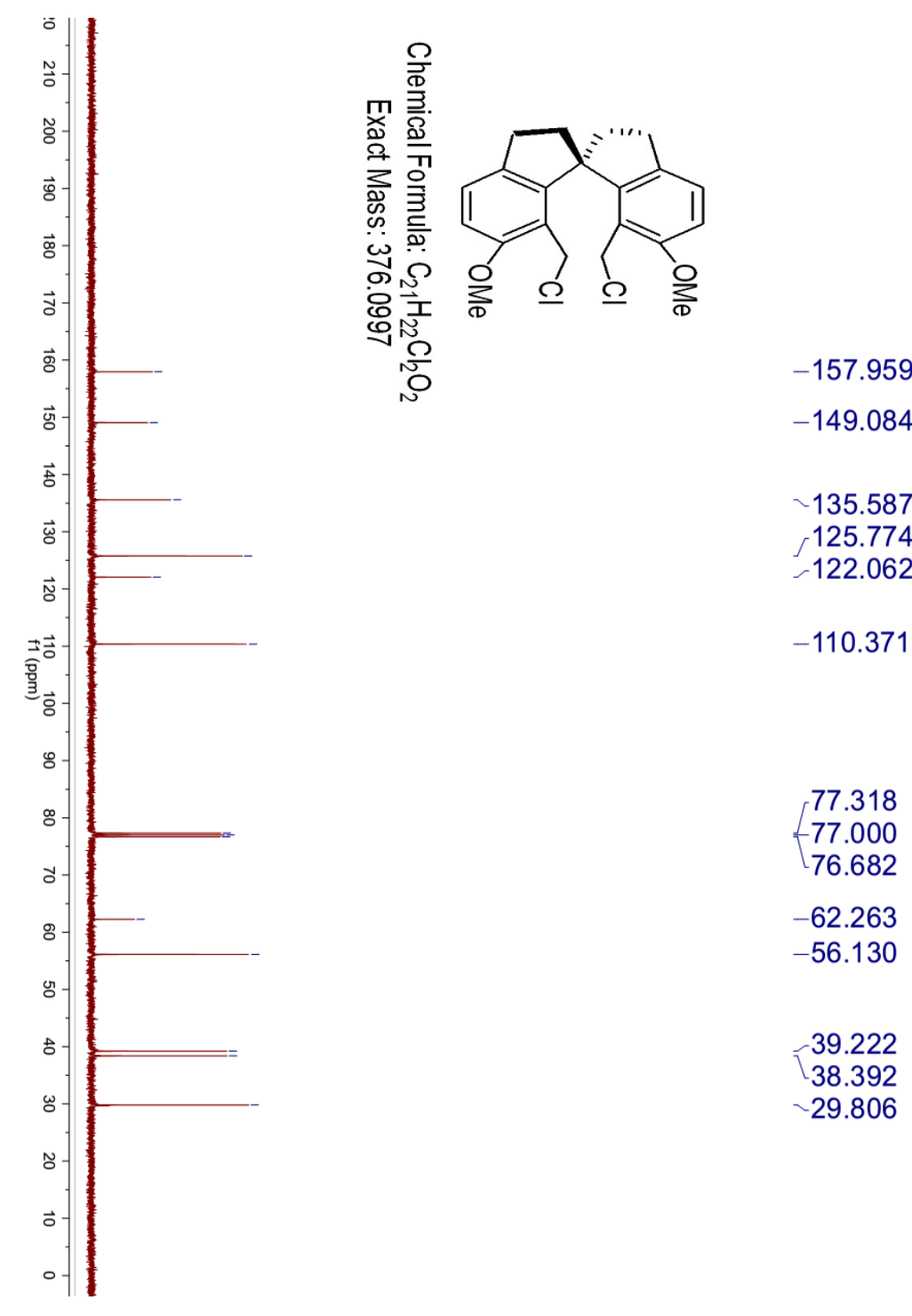

135.587
125.774

122.062

$-110.371$

77.318

$-77.000$

76.682

$-62.263$

$-56.130$

$-39.222$

38.392

$-29.806$ 


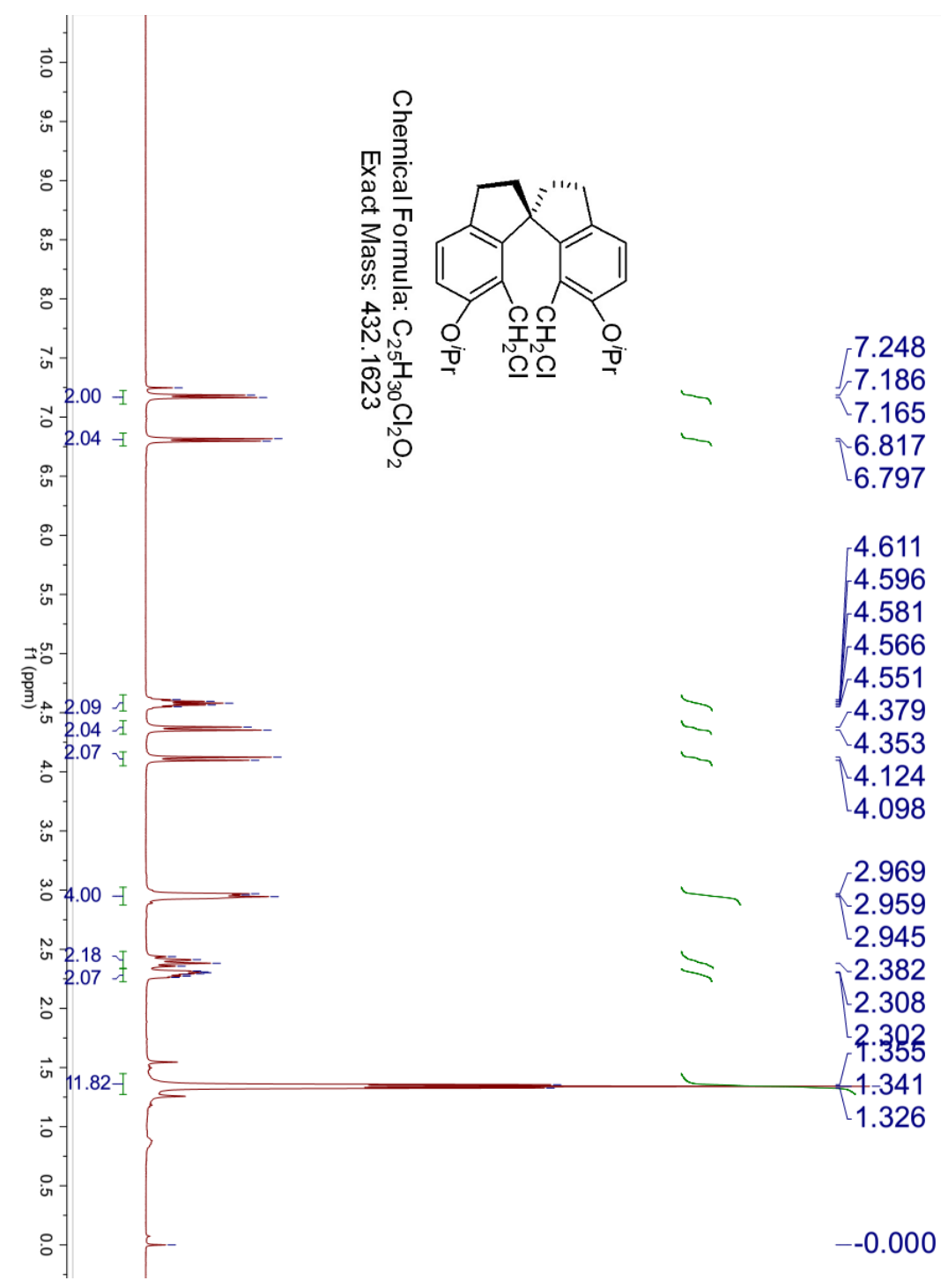




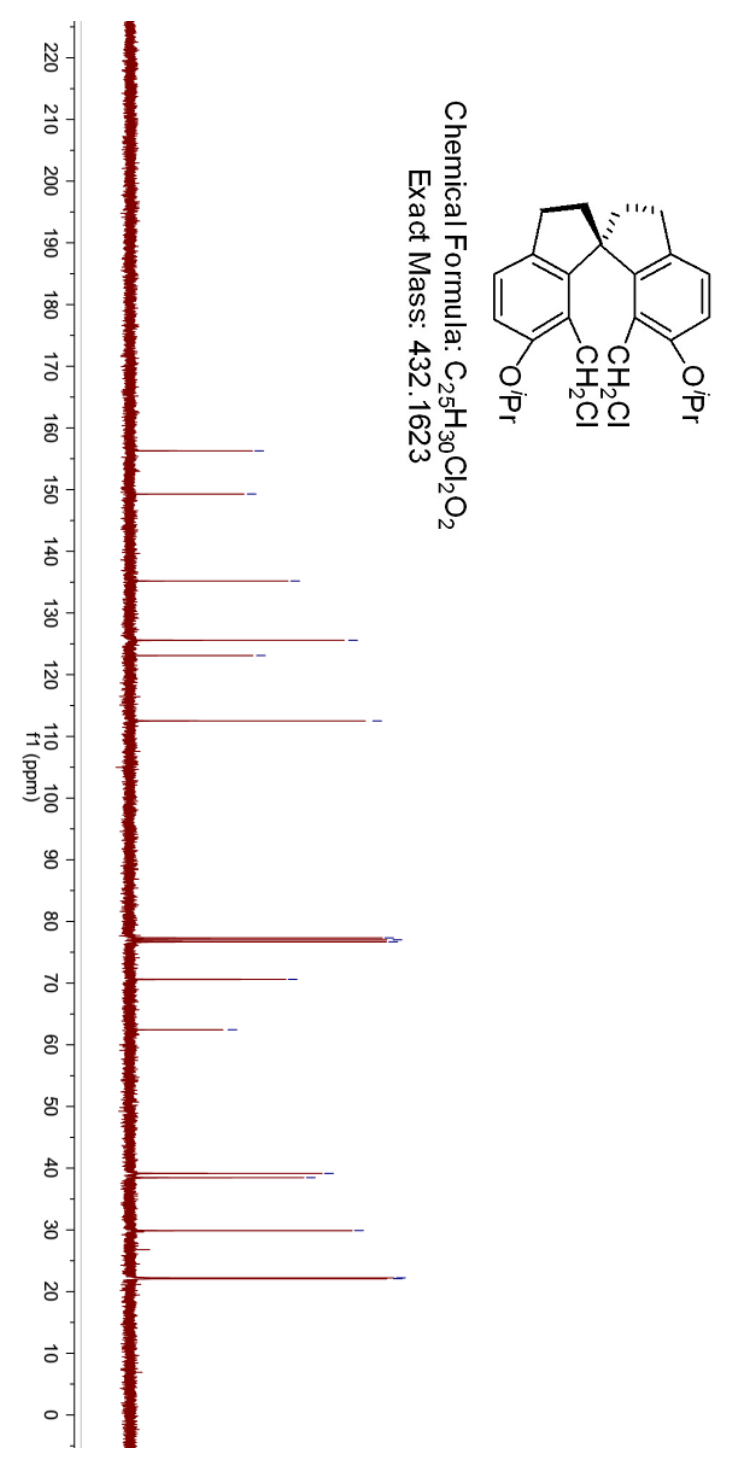

135.191

125.584

$\backslash 123.102$

$-112.512$

77.325

77.007

76.690

70.615

62.437

39.160

38.472

$-29.880$

22.222

22.087 


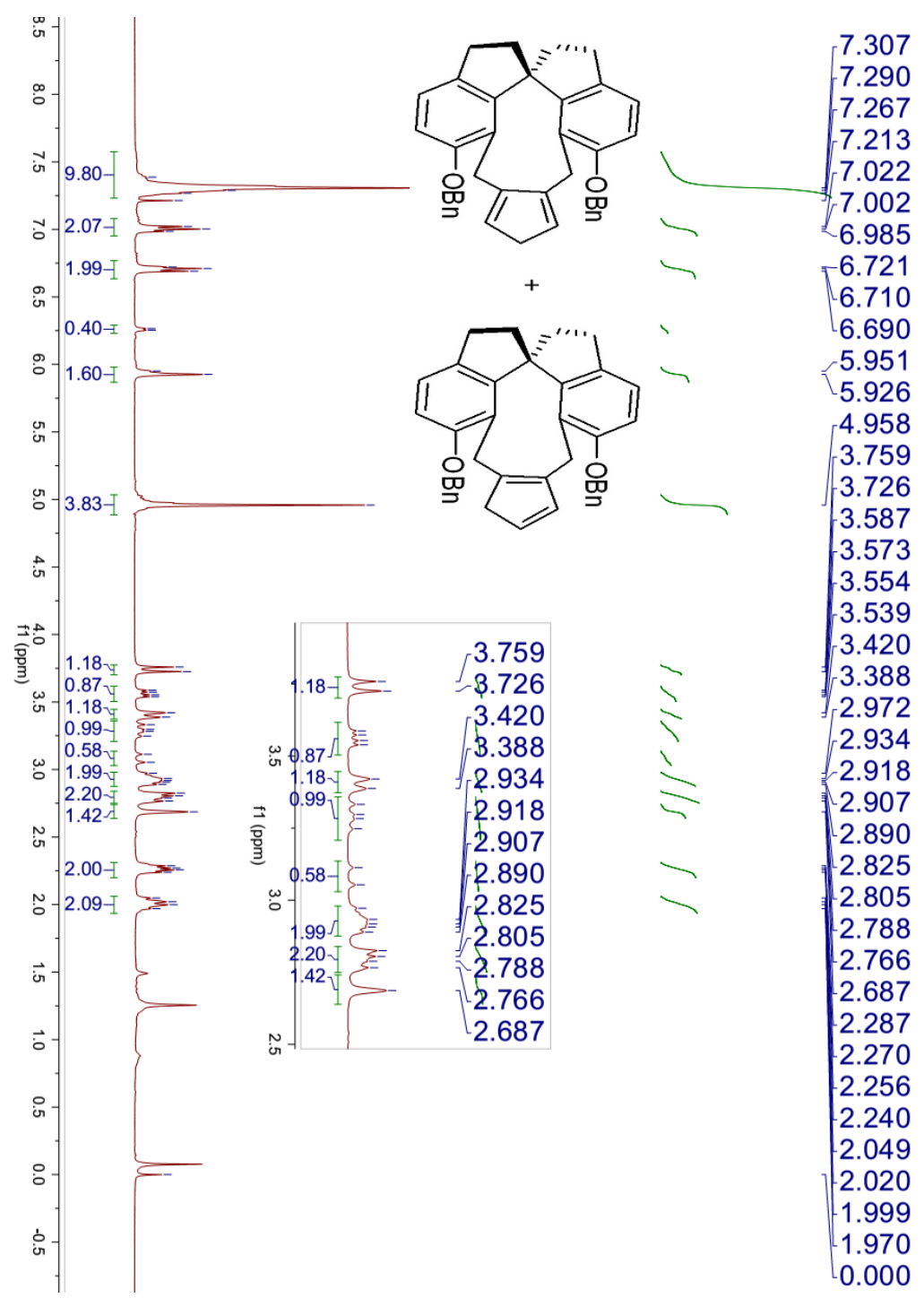




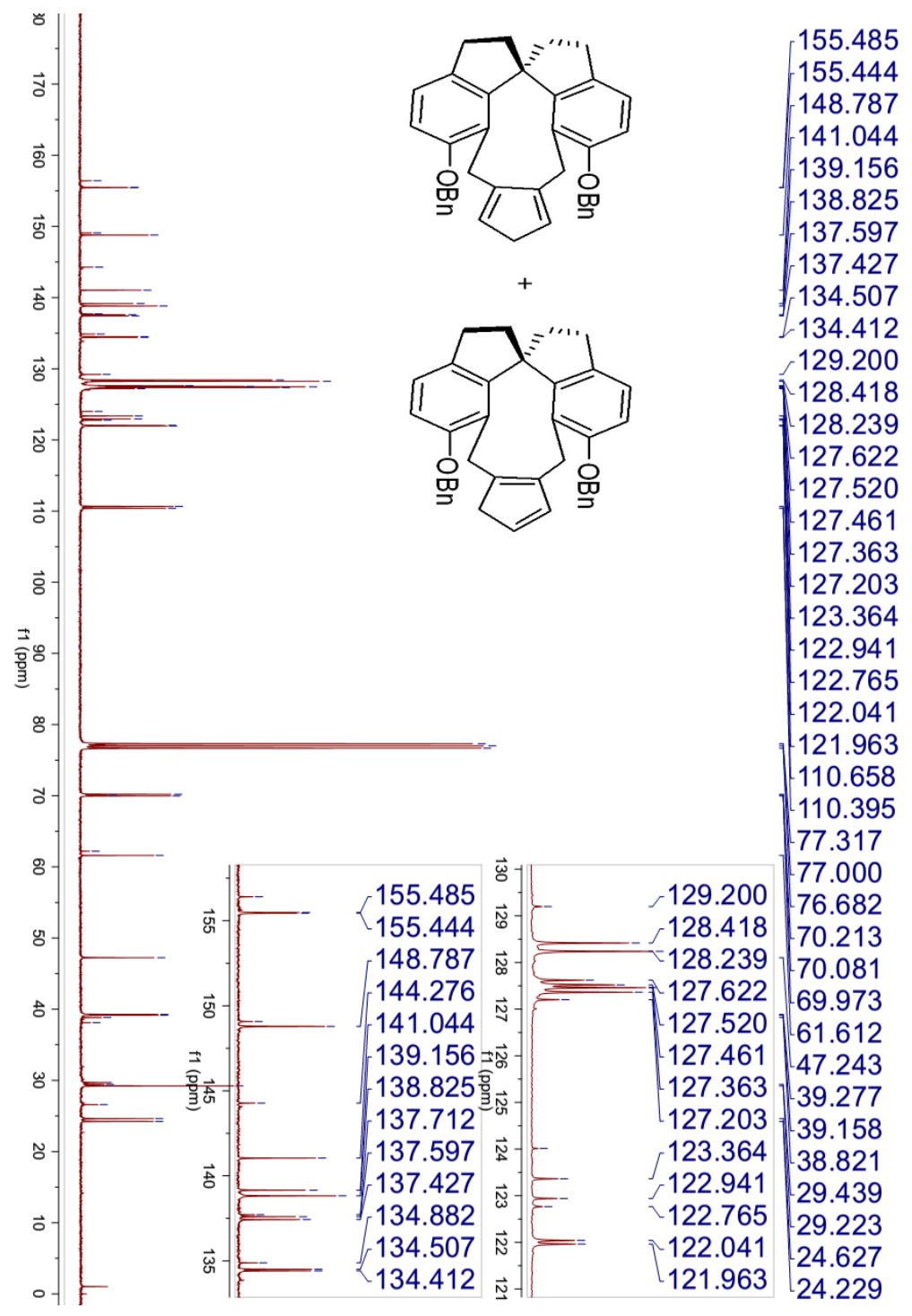




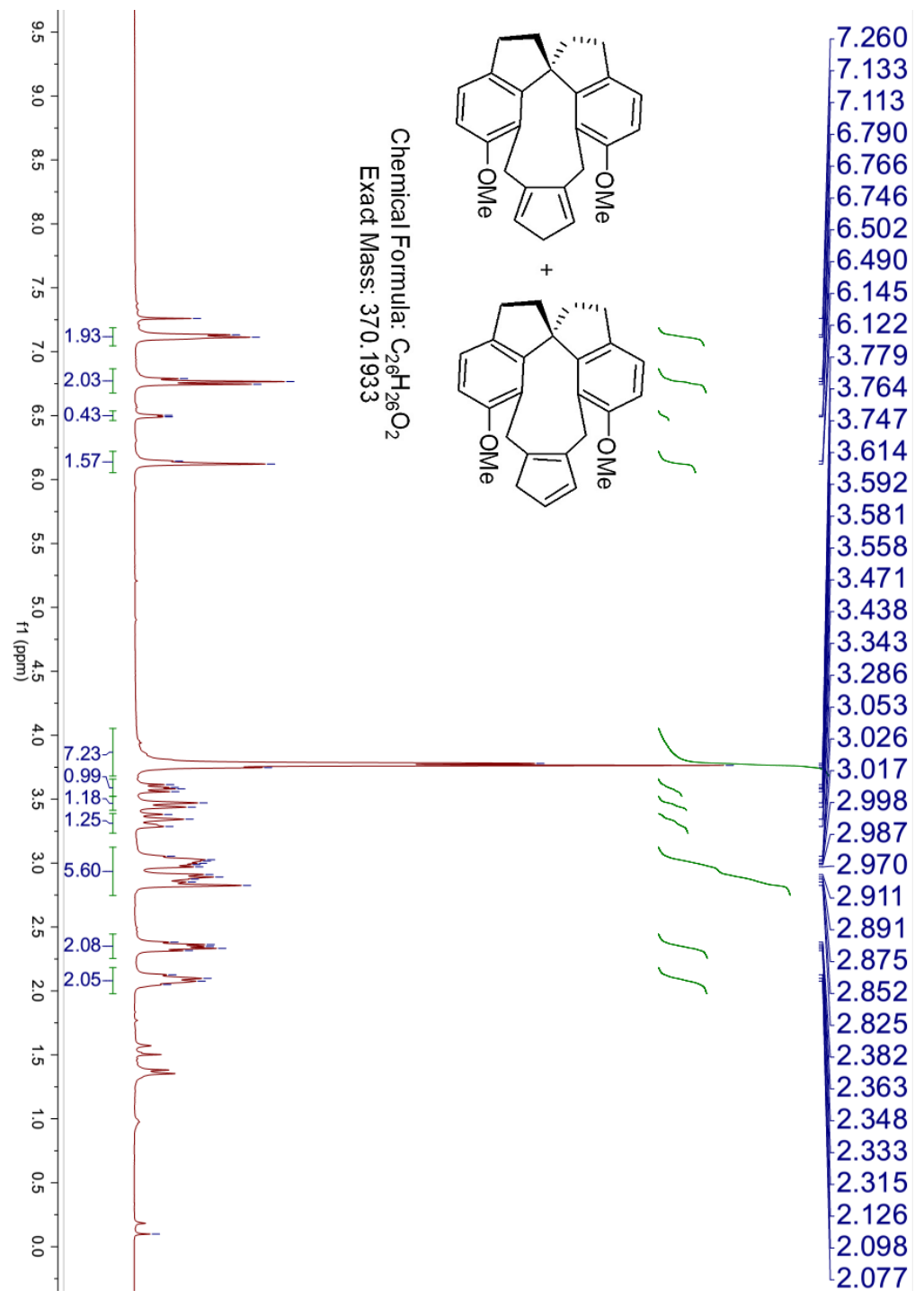




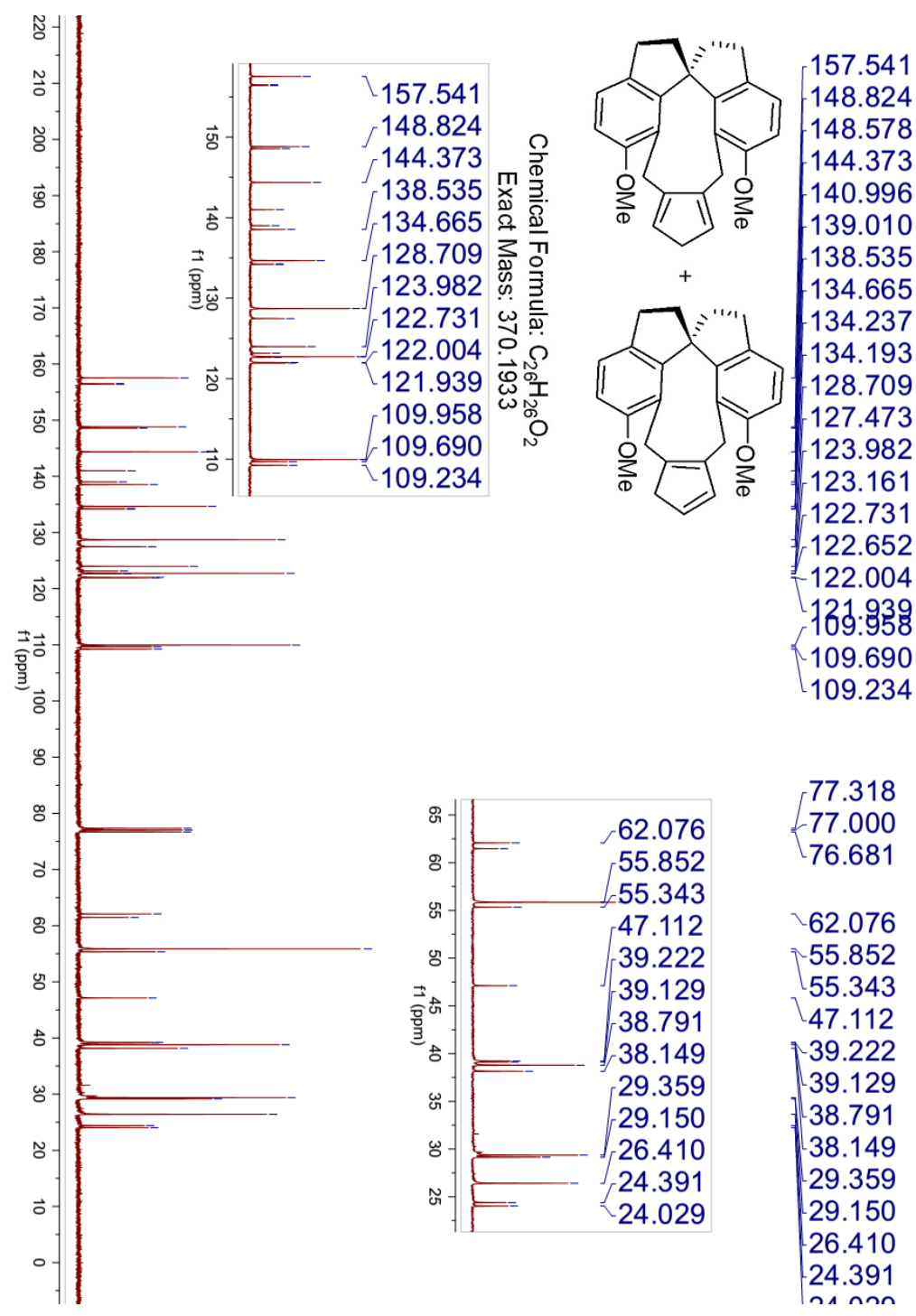




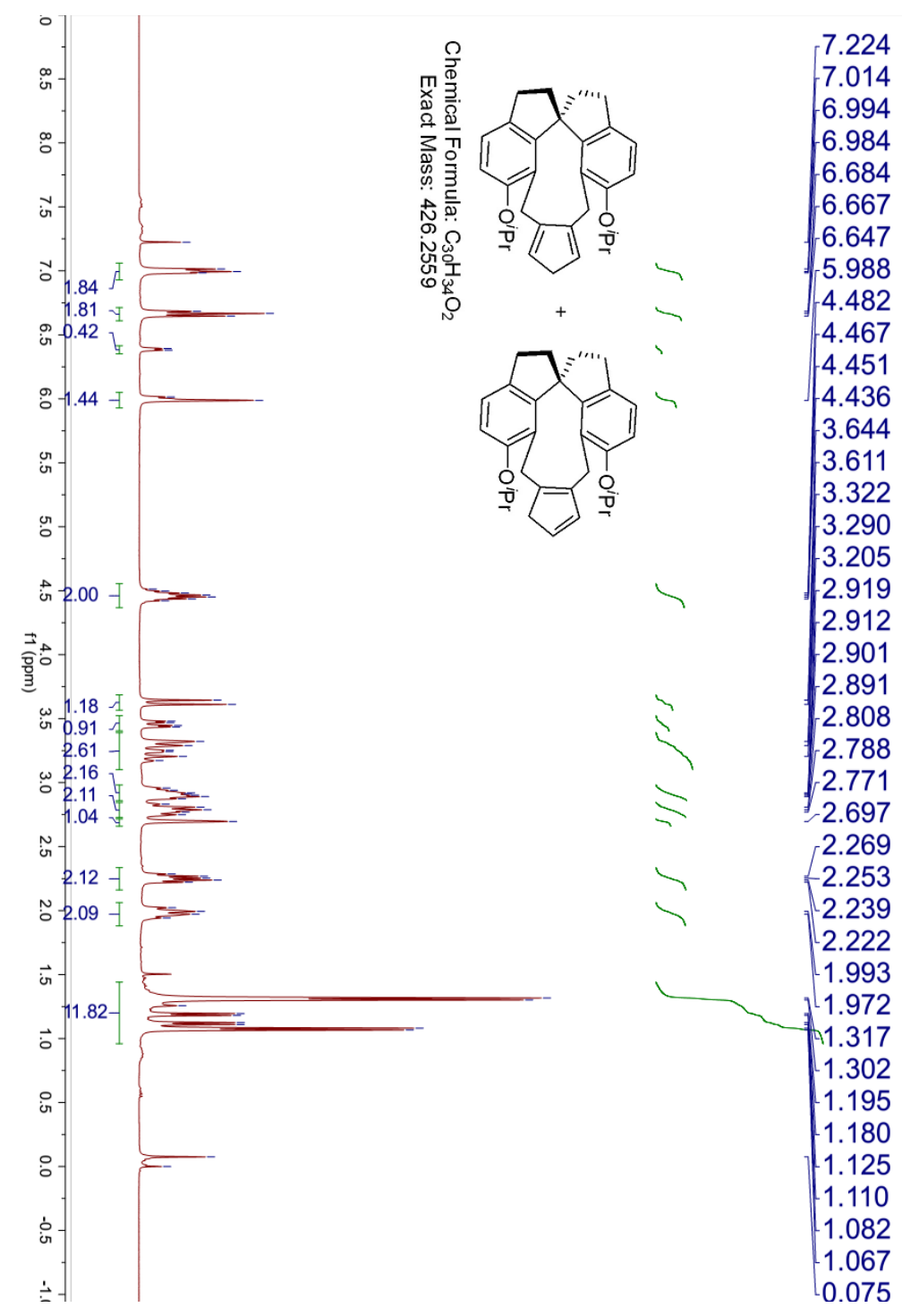




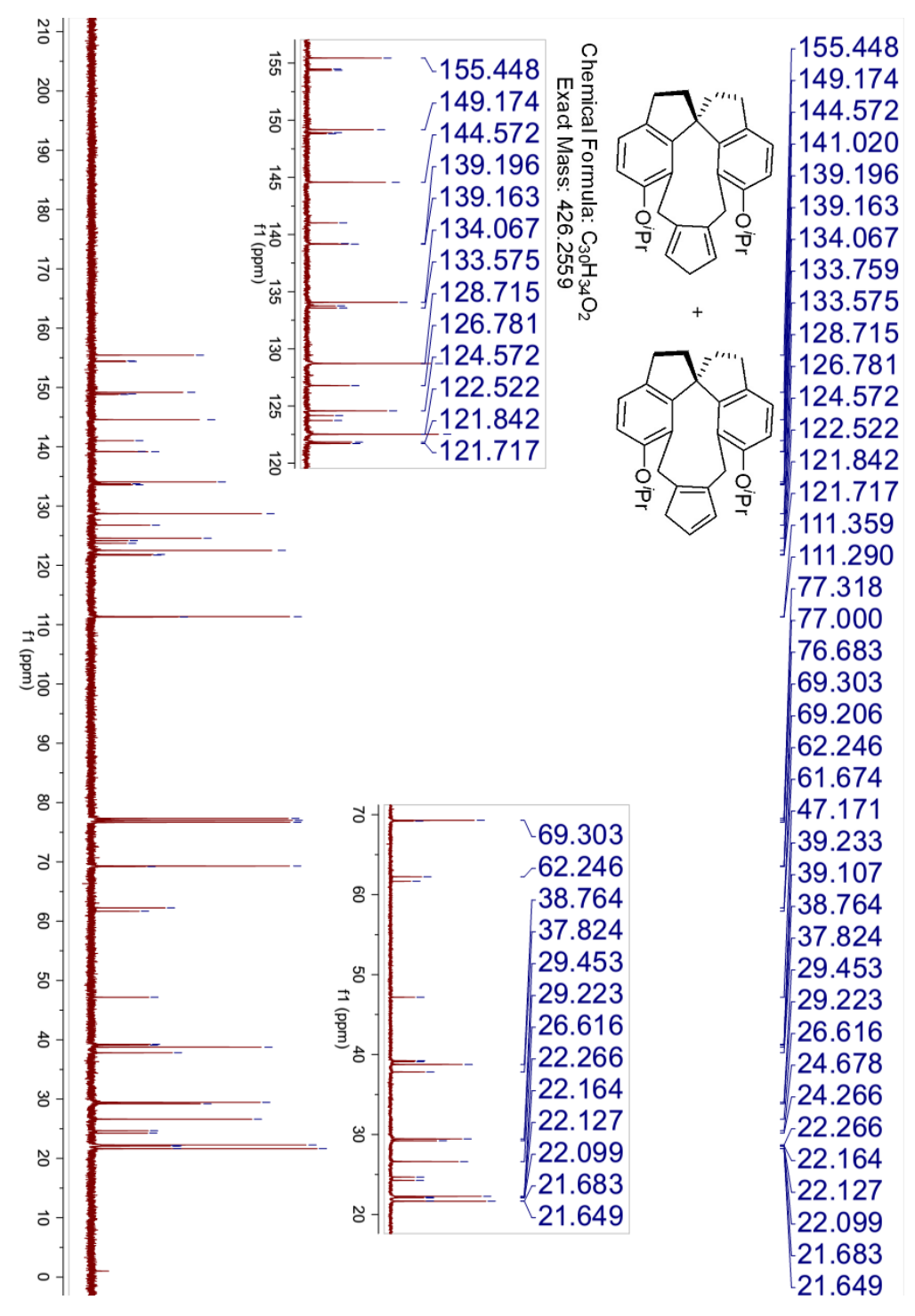



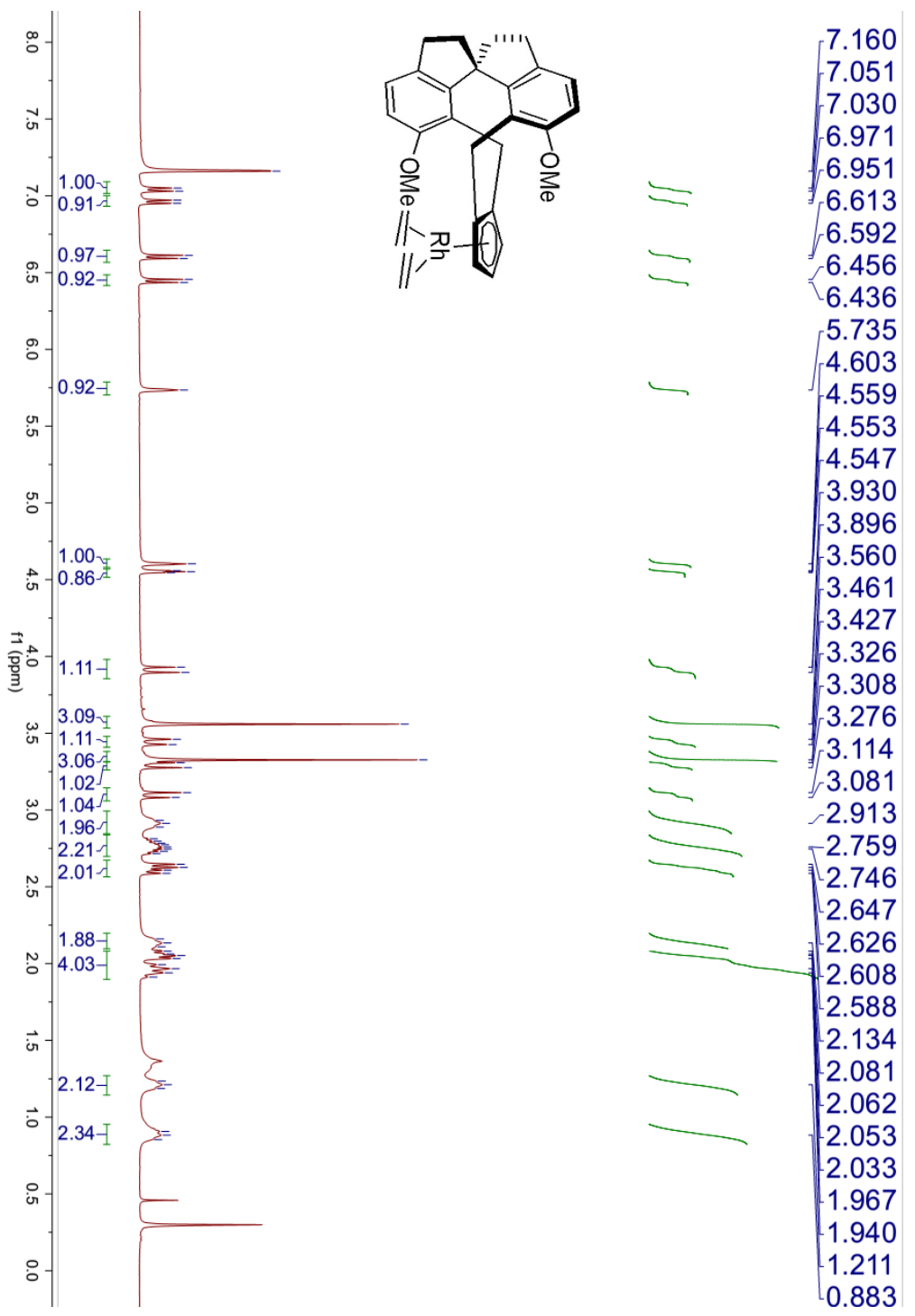


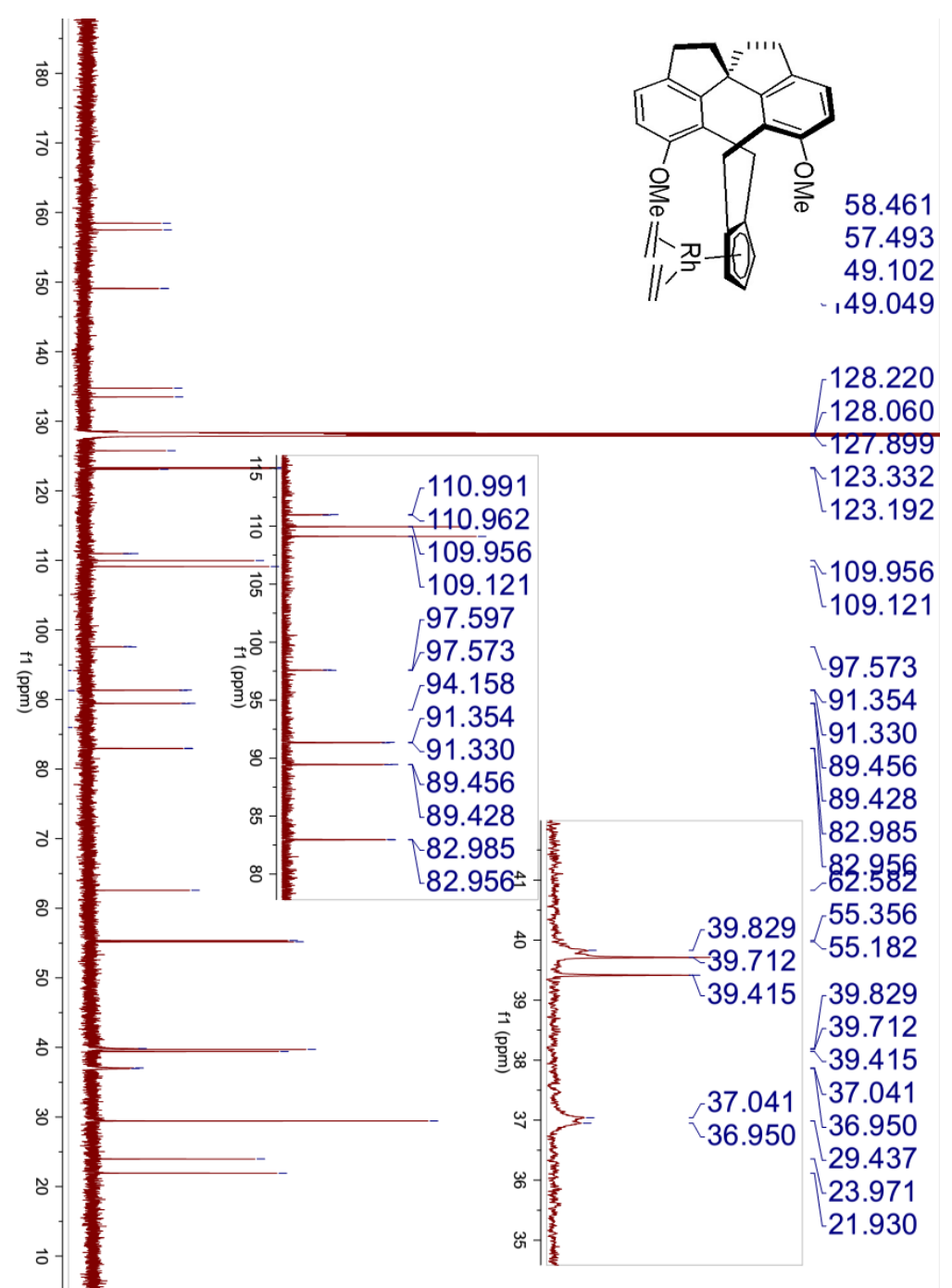




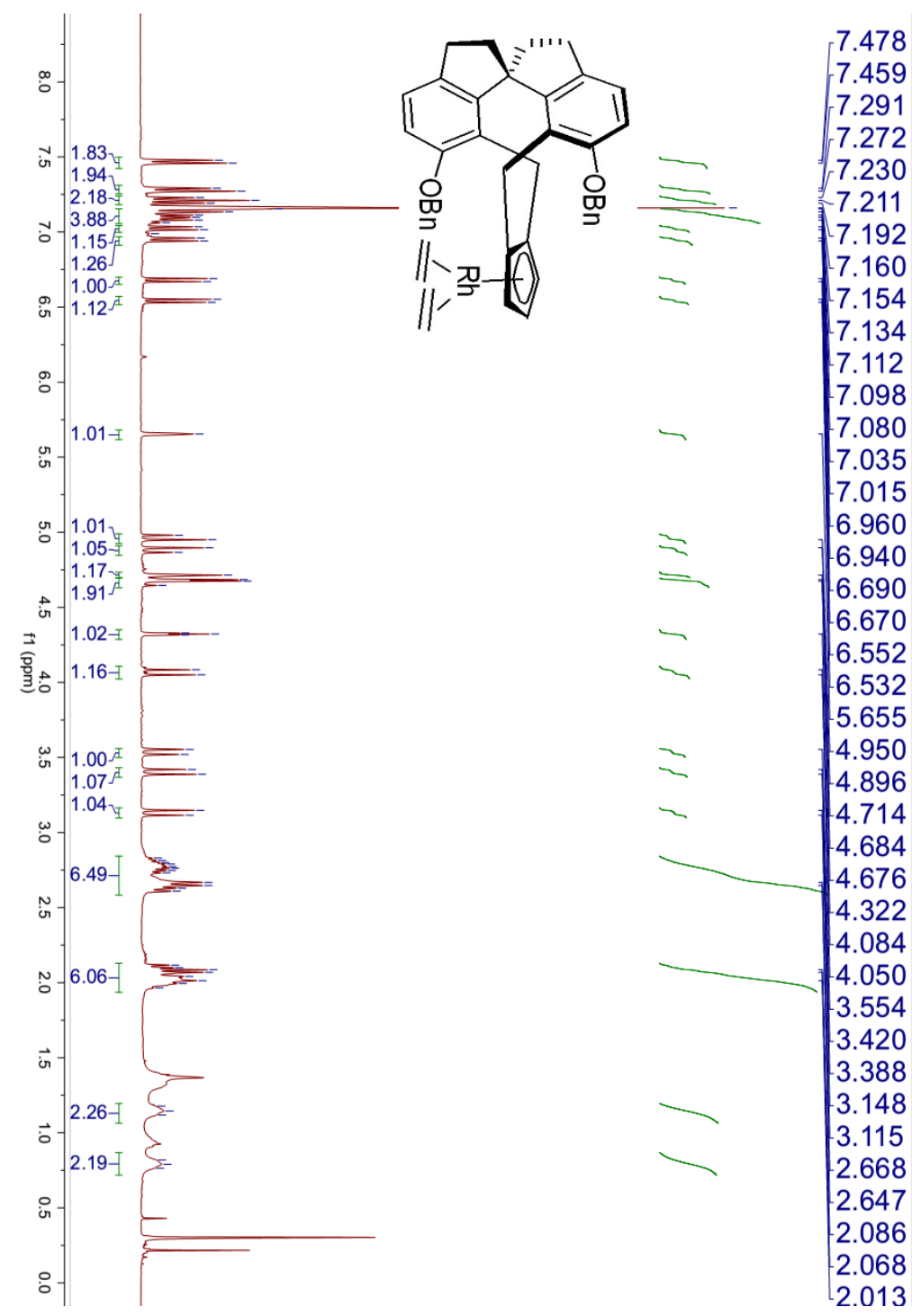




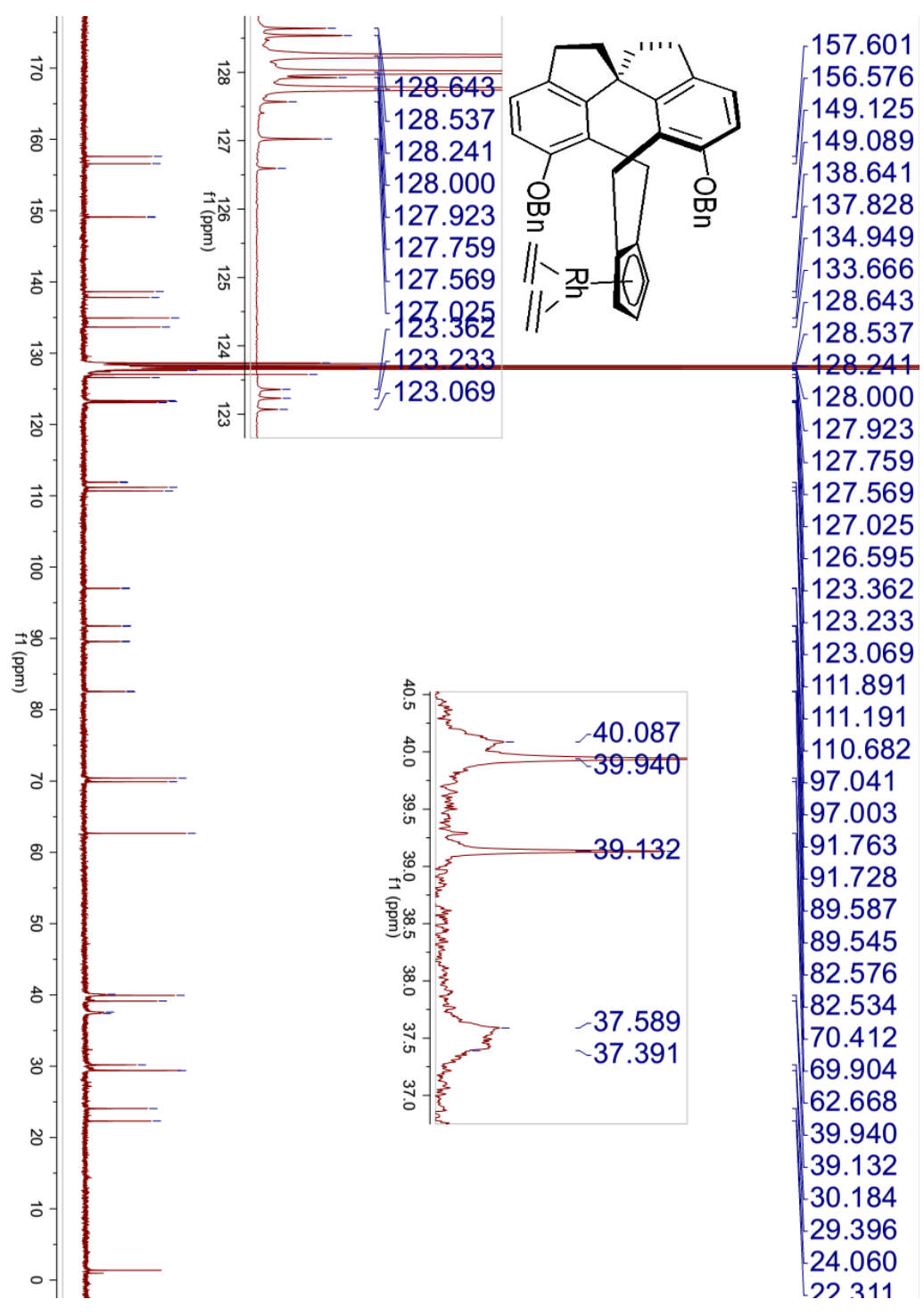



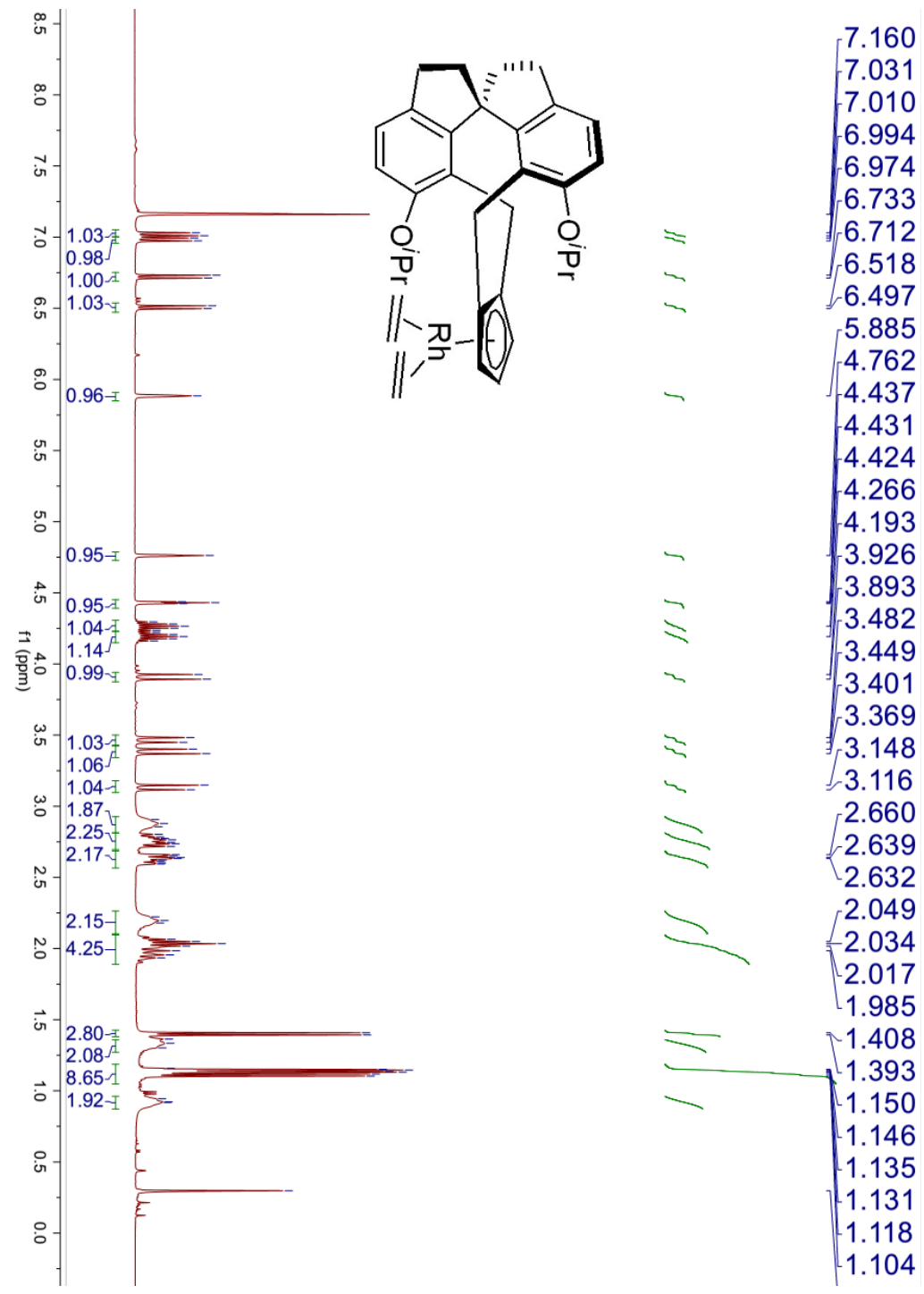


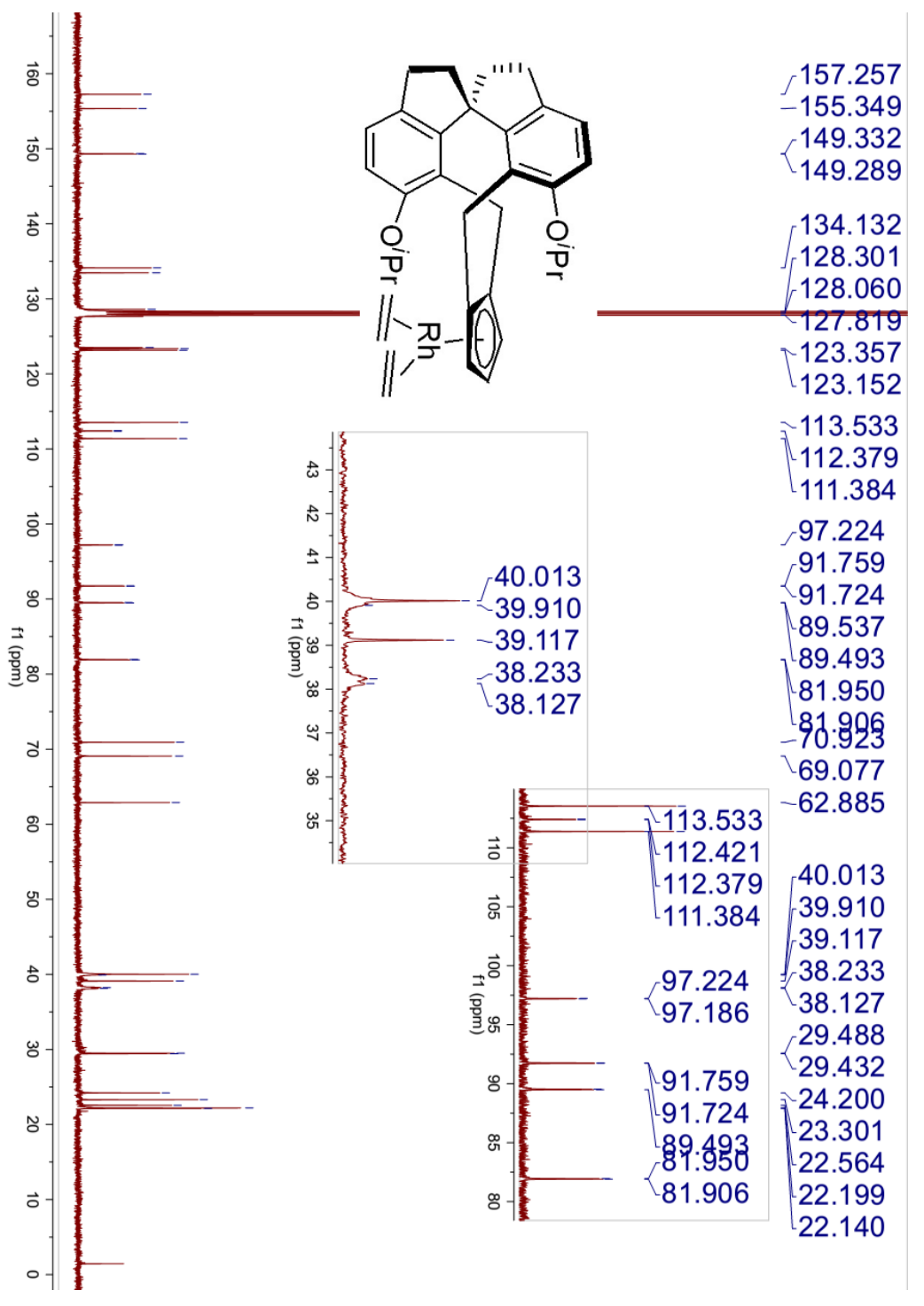




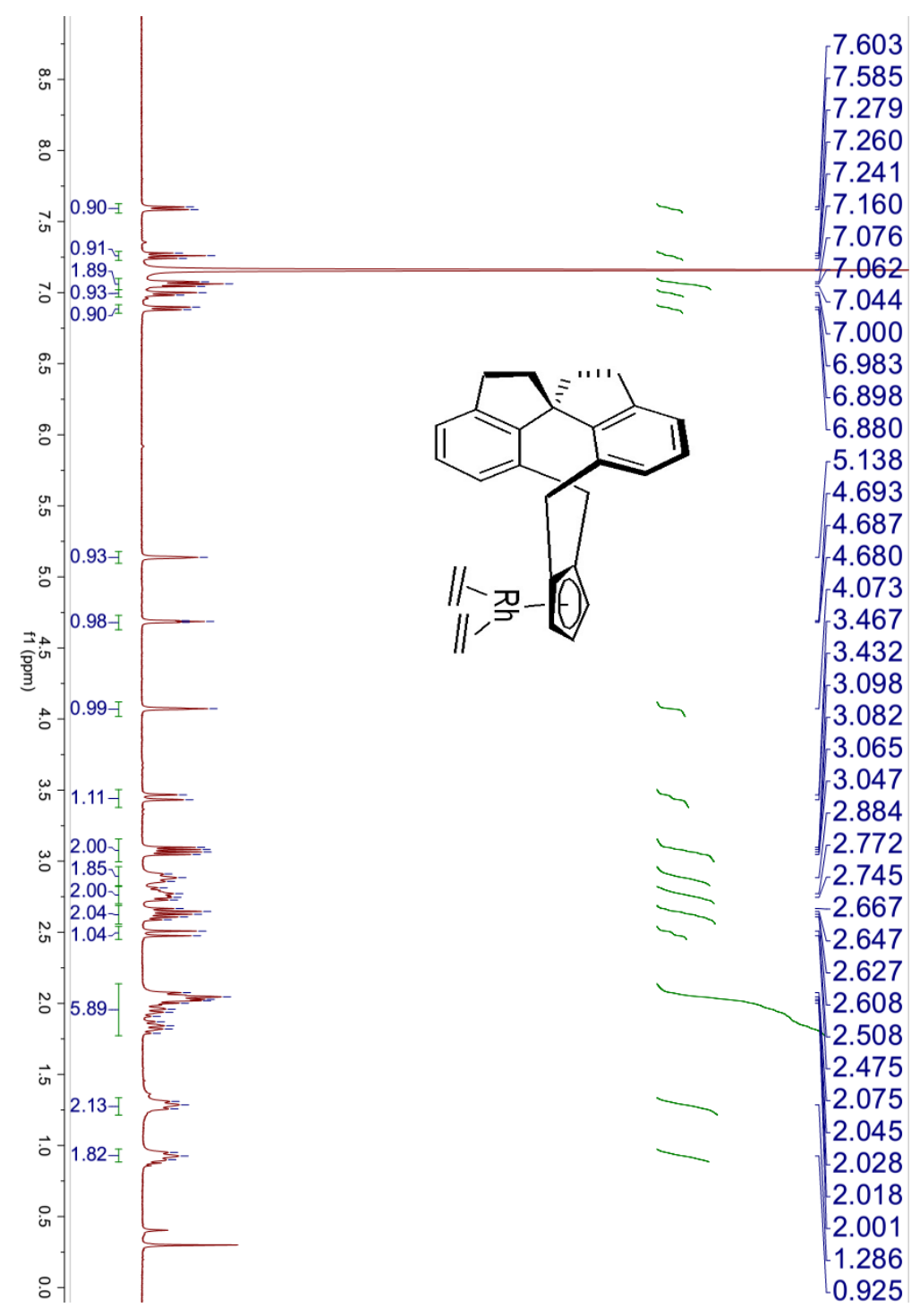




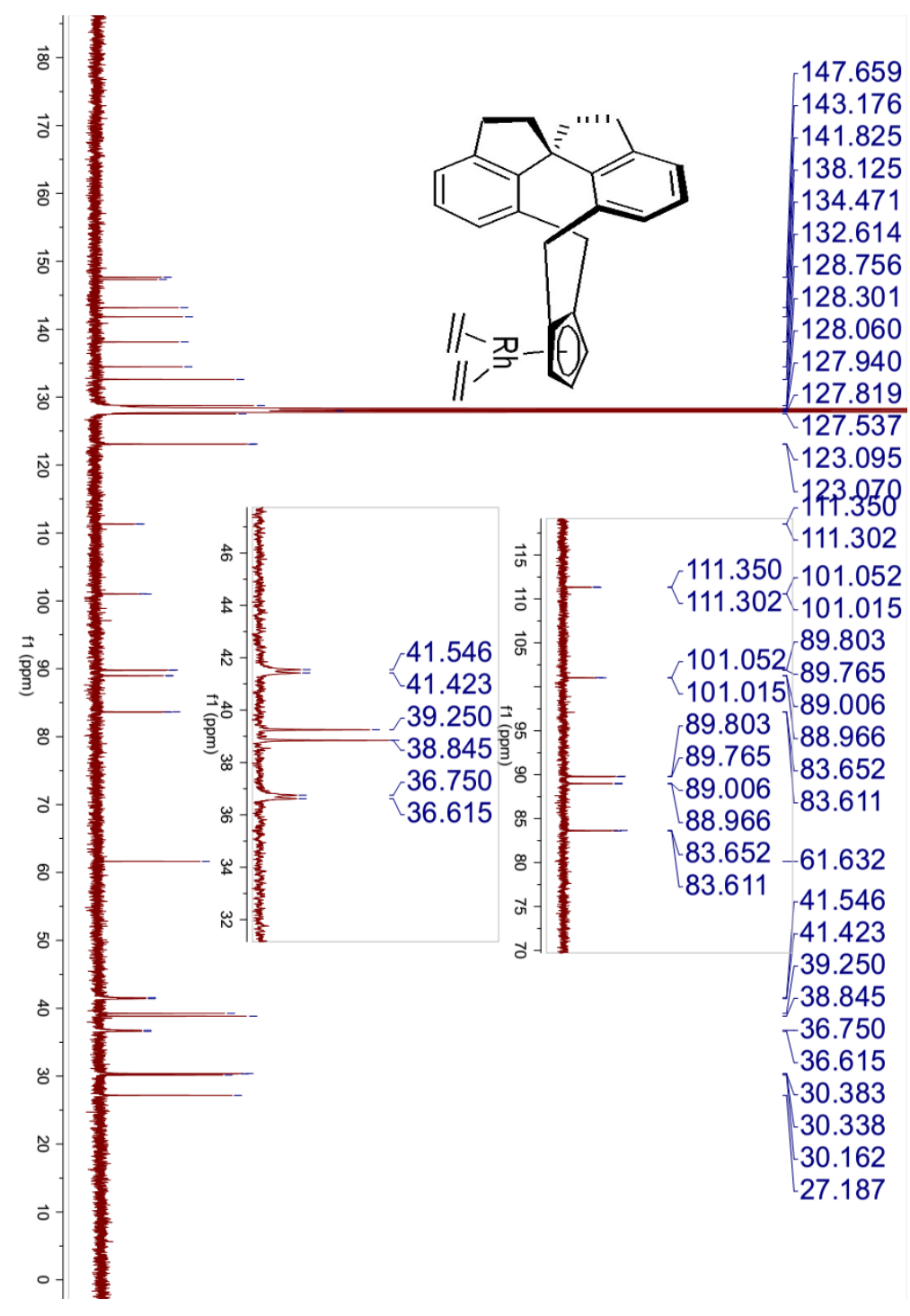




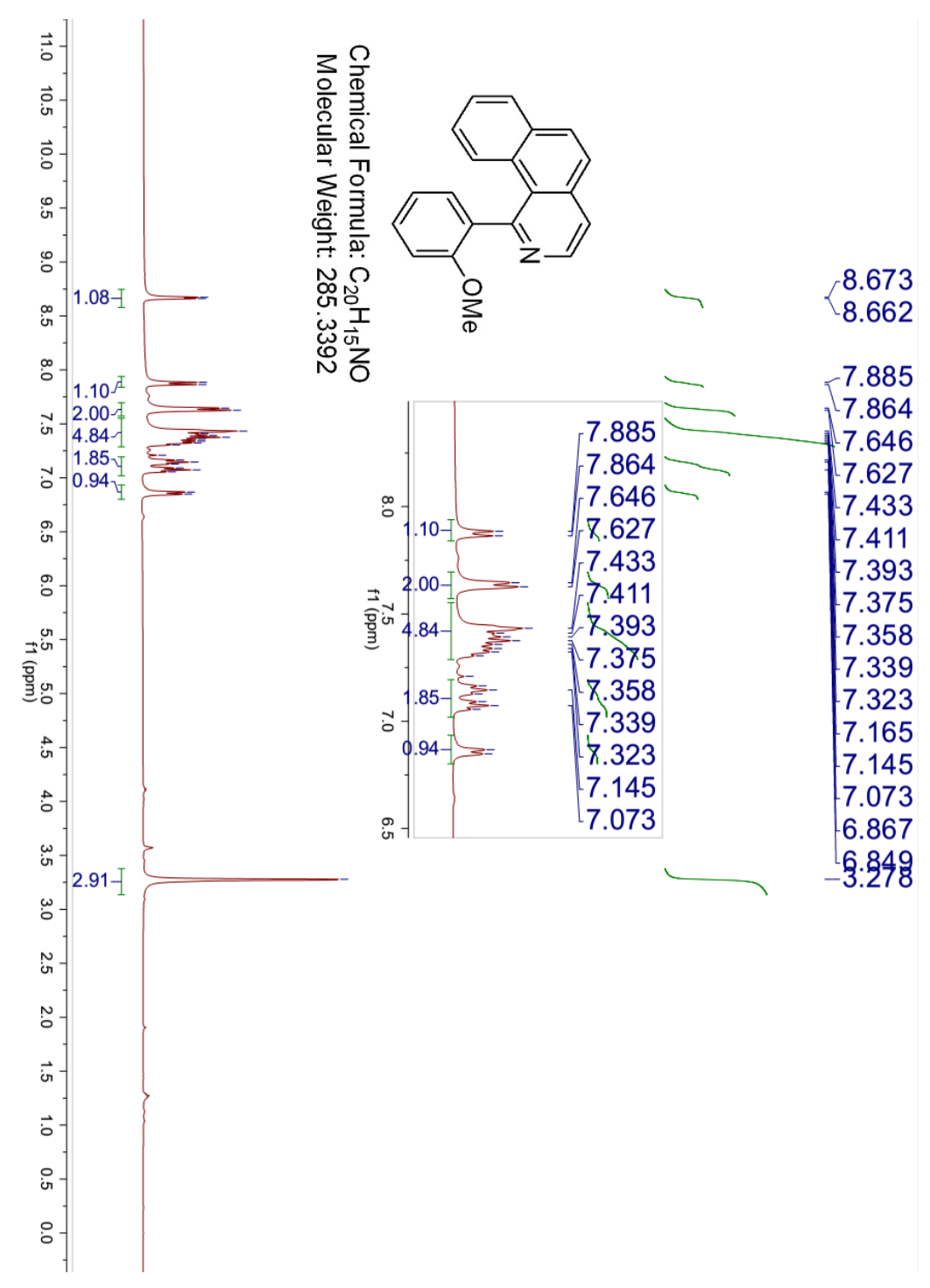



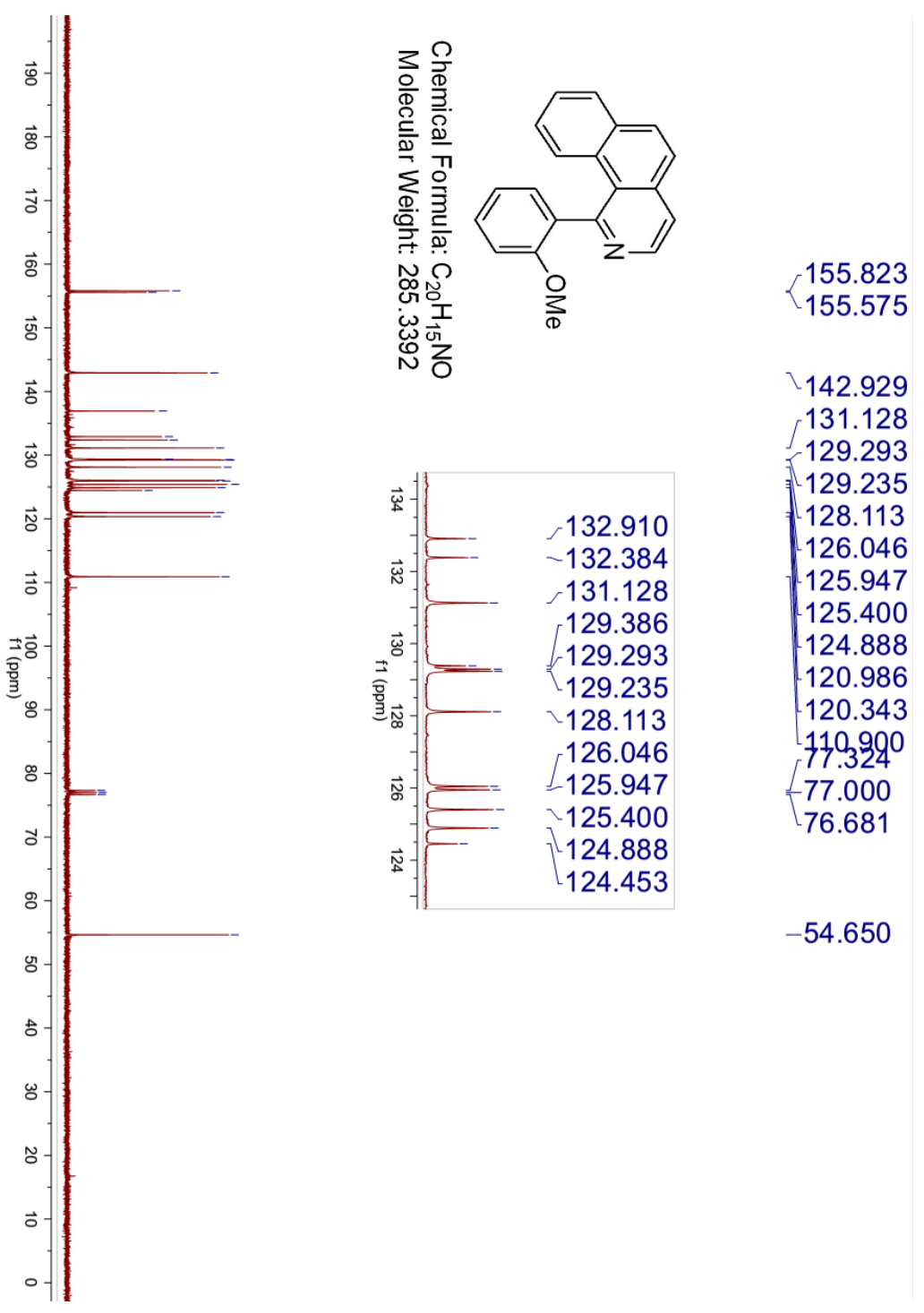


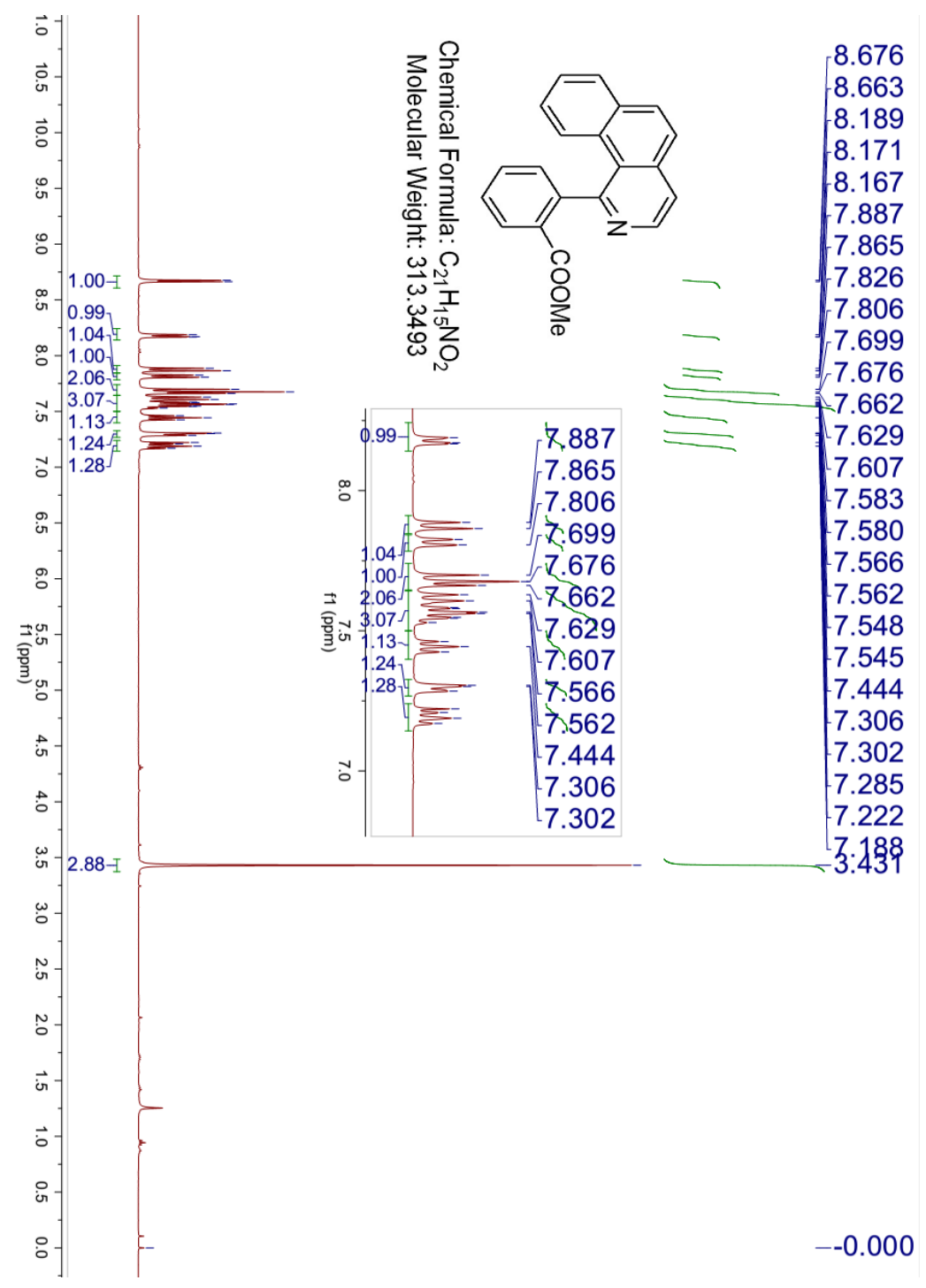




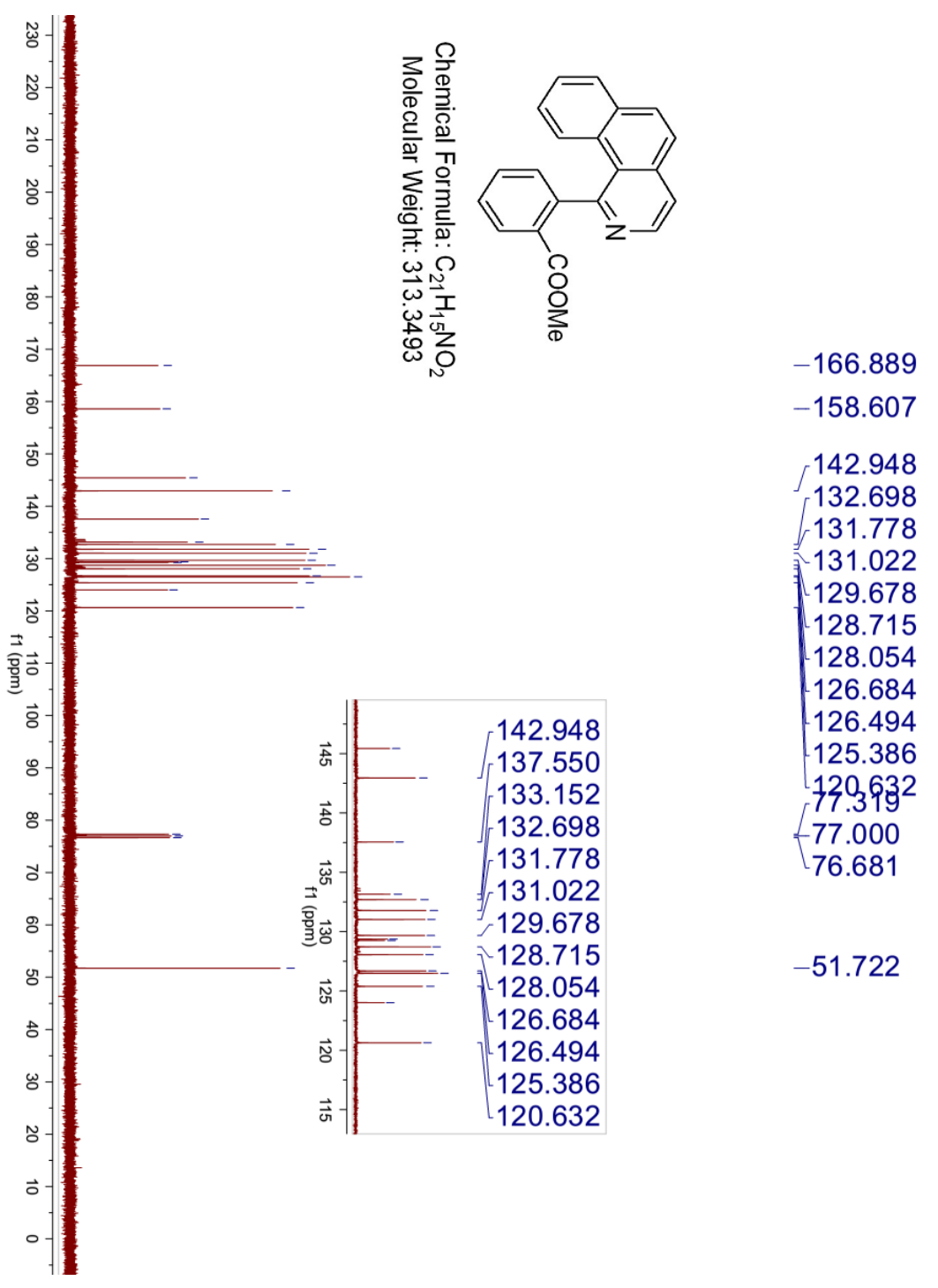




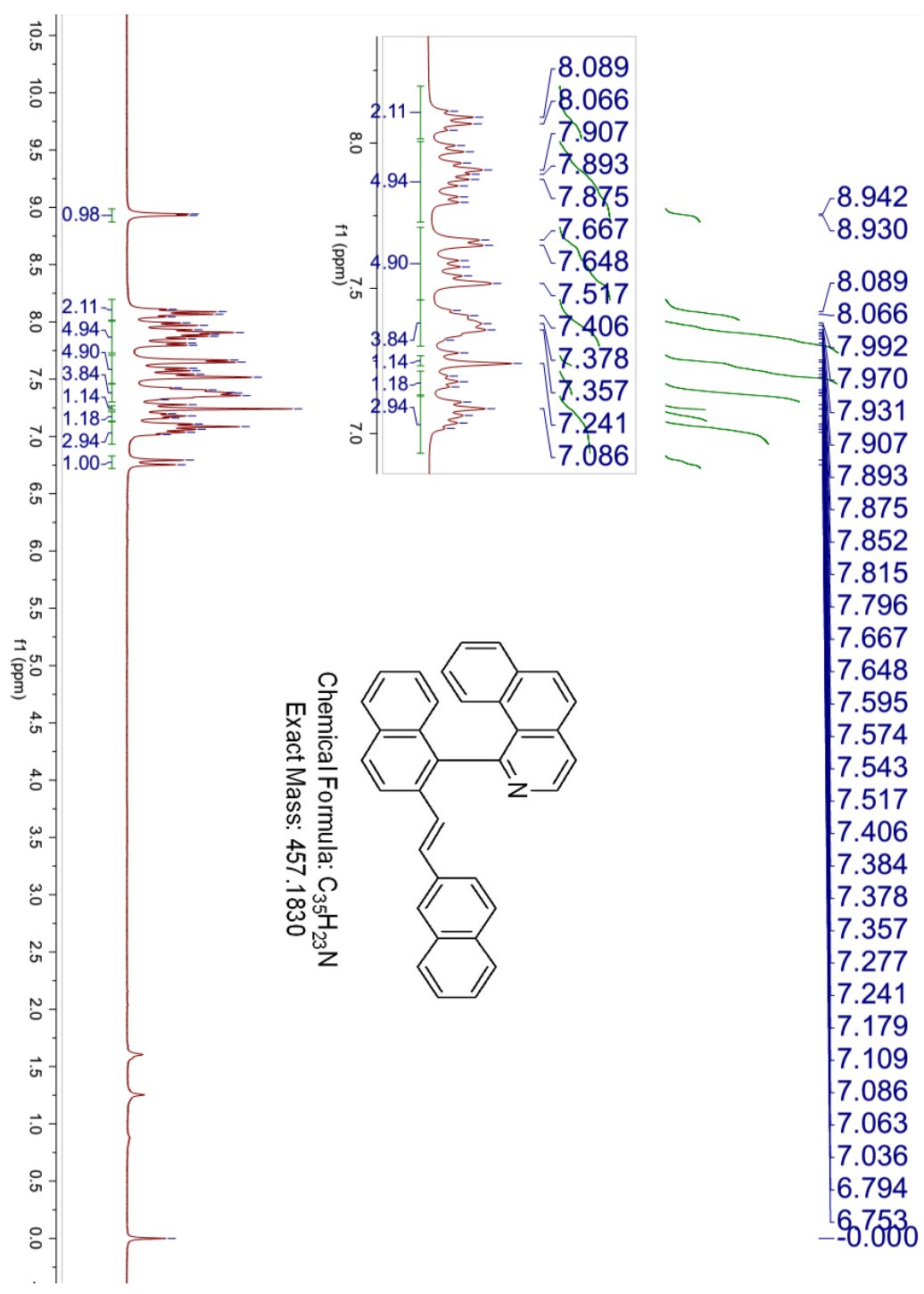




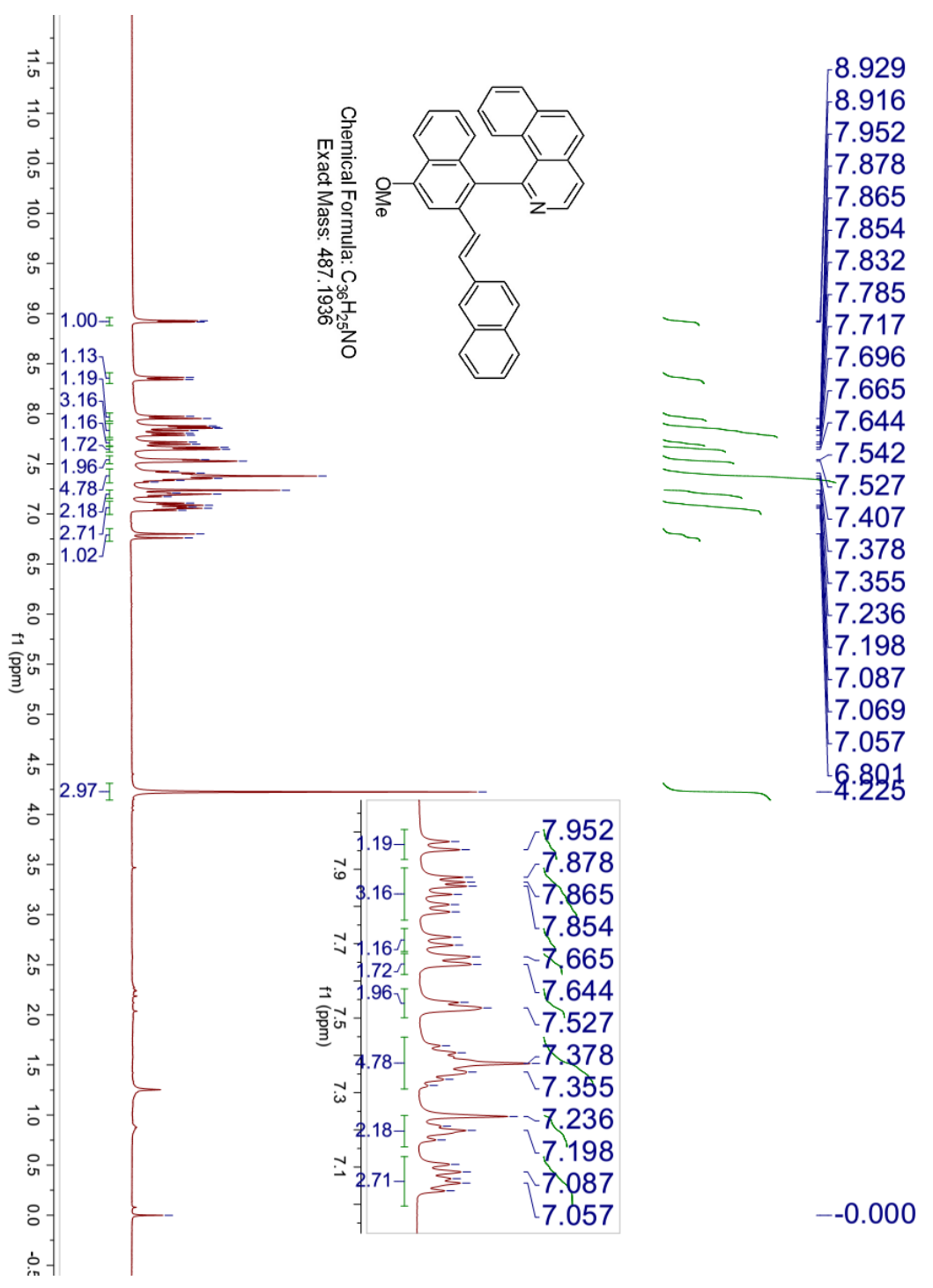




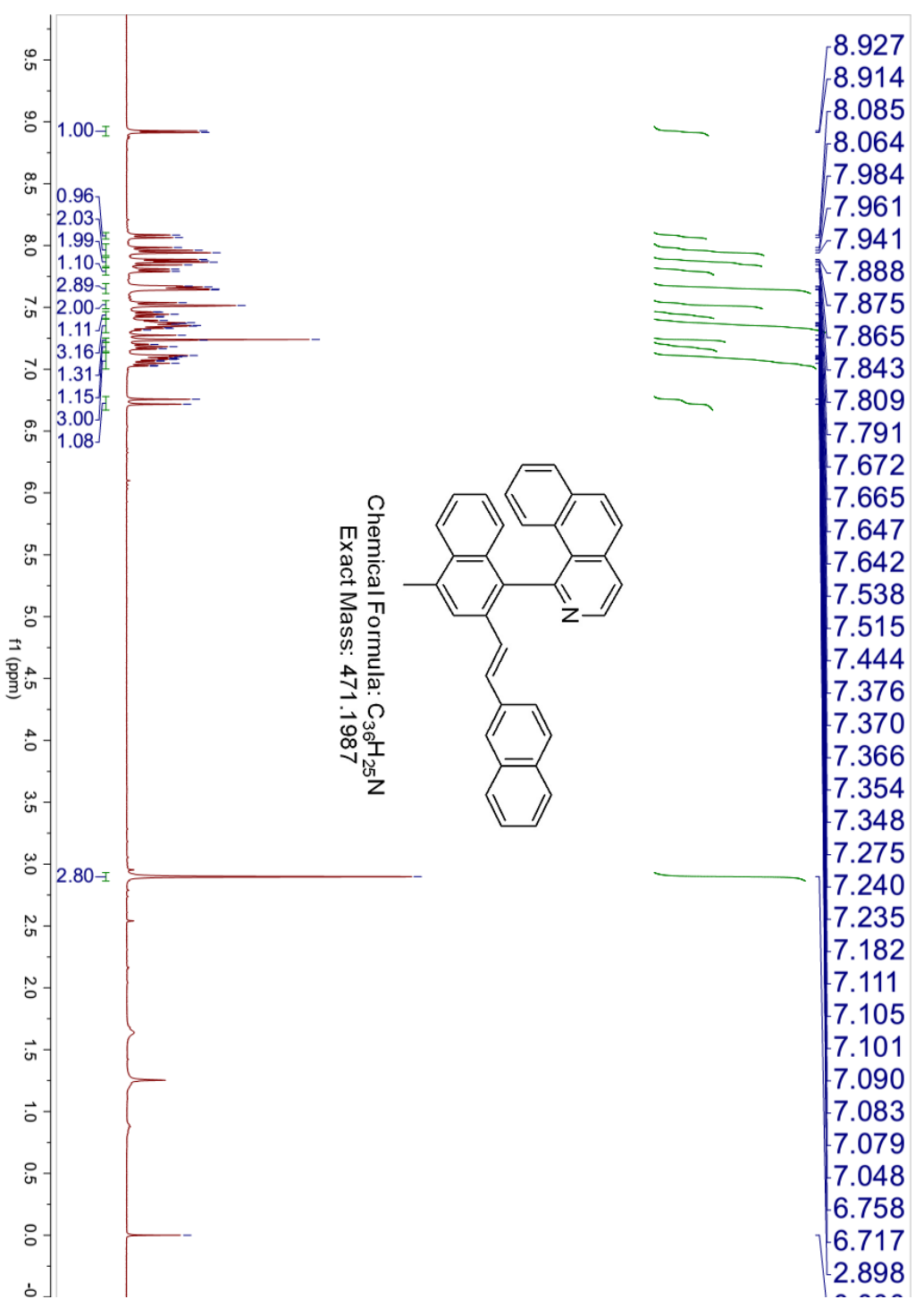




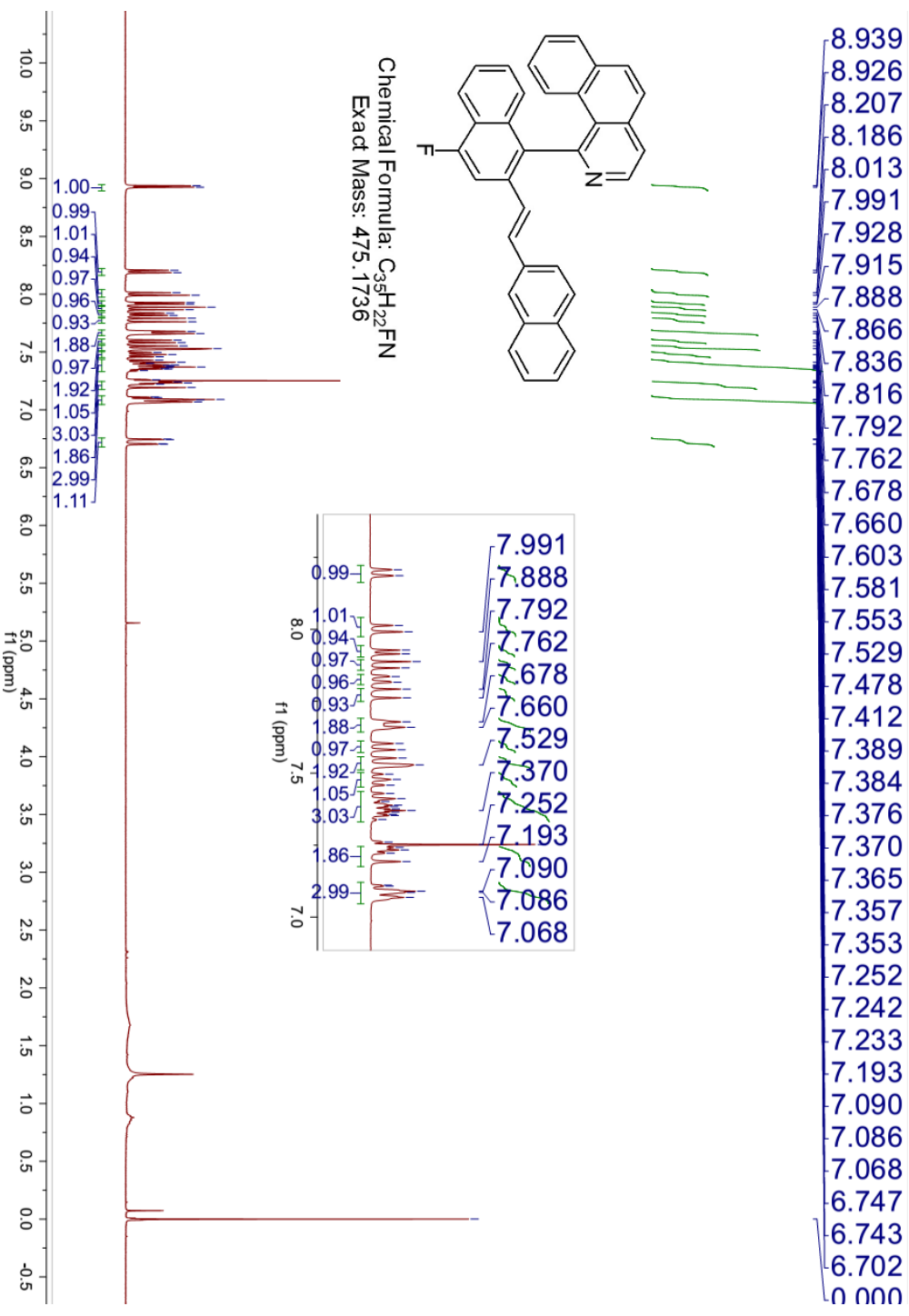




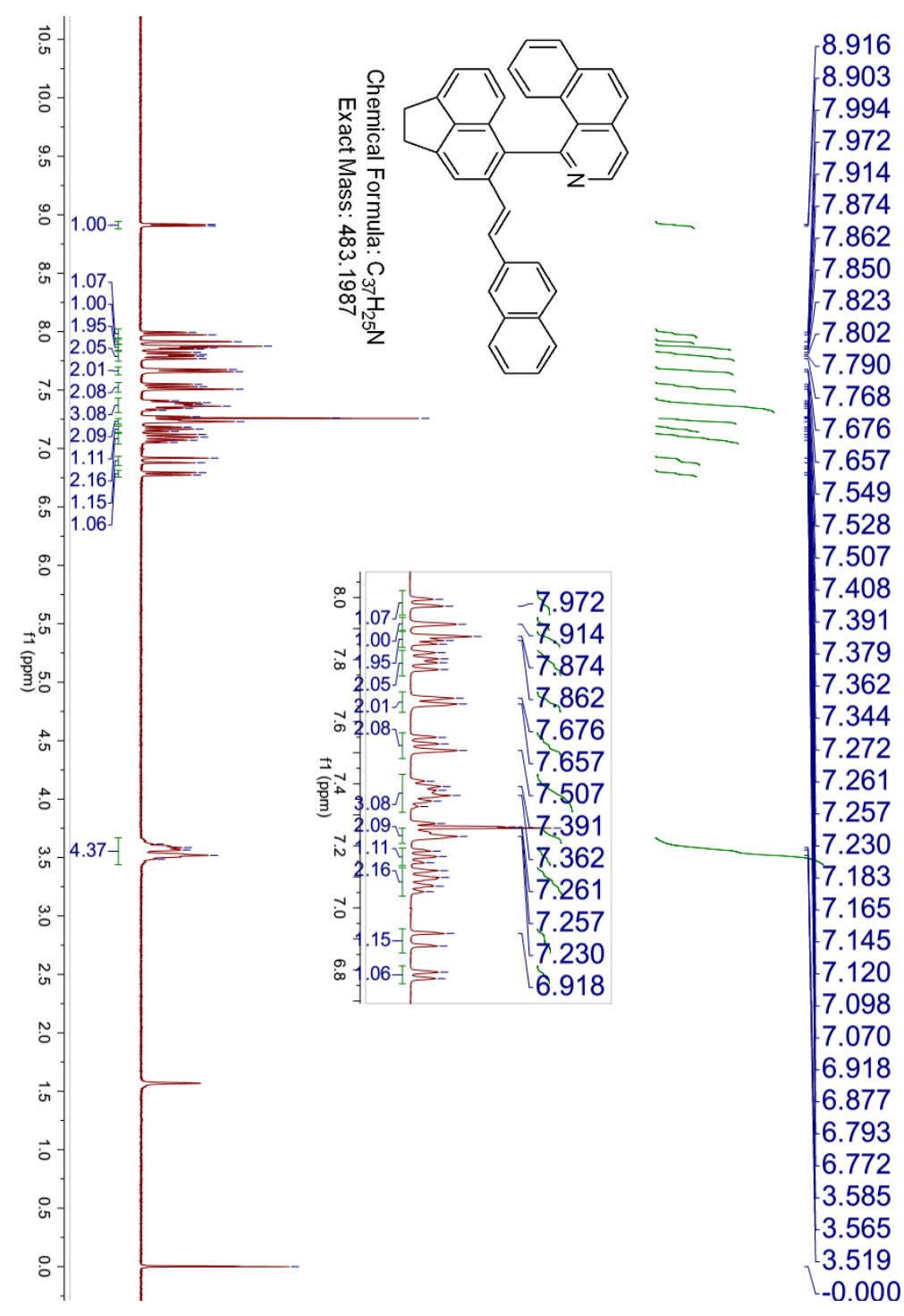




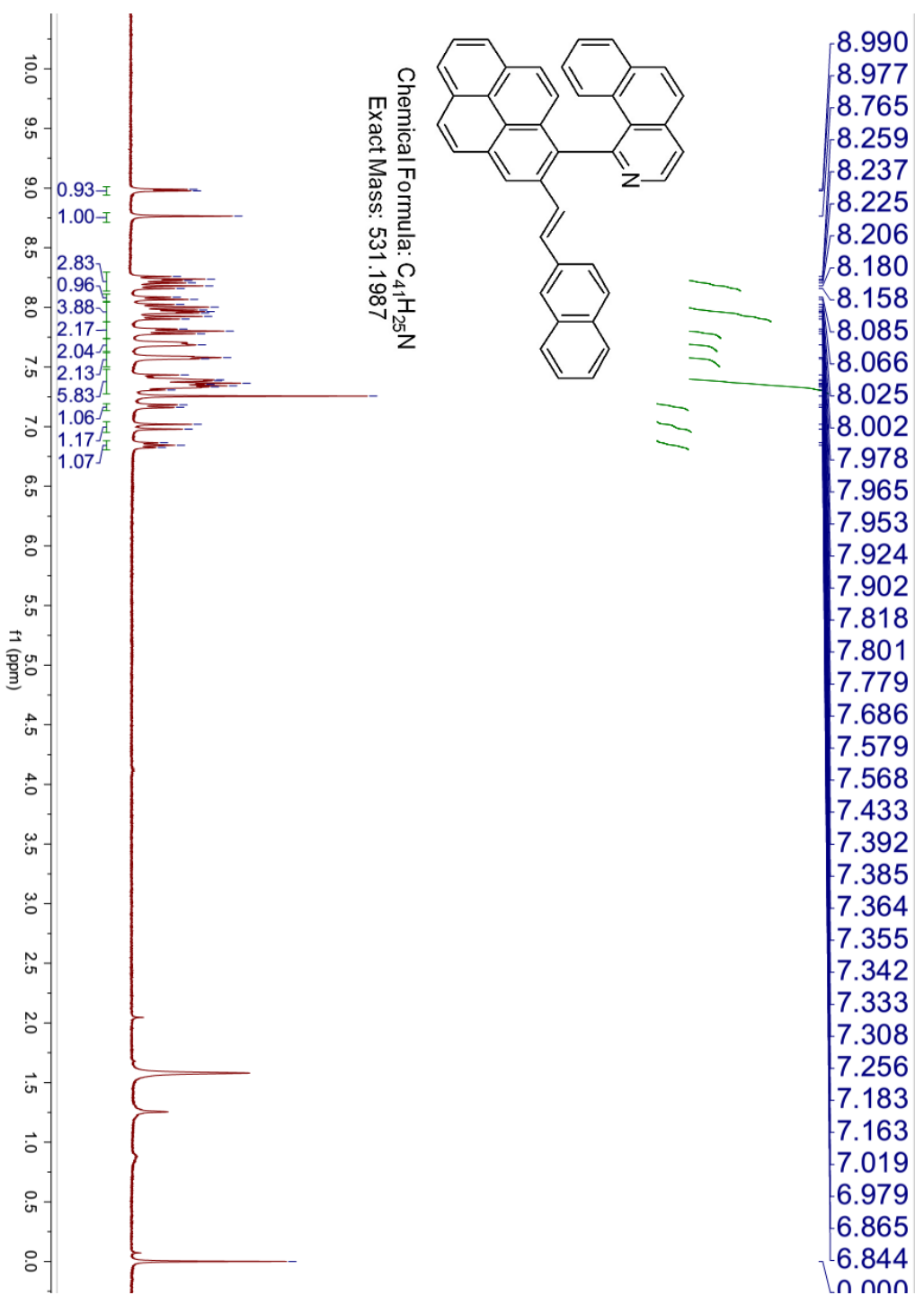




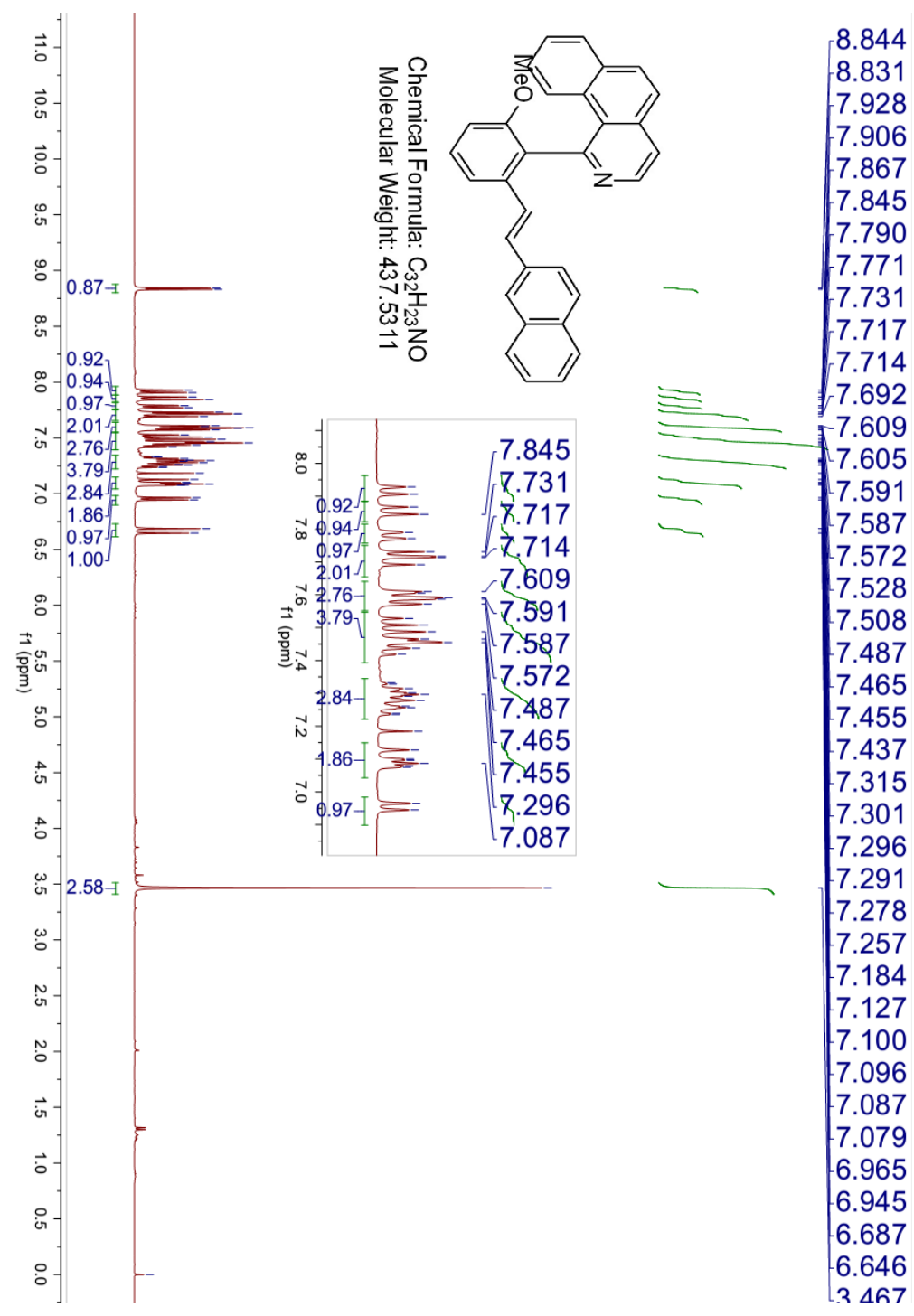




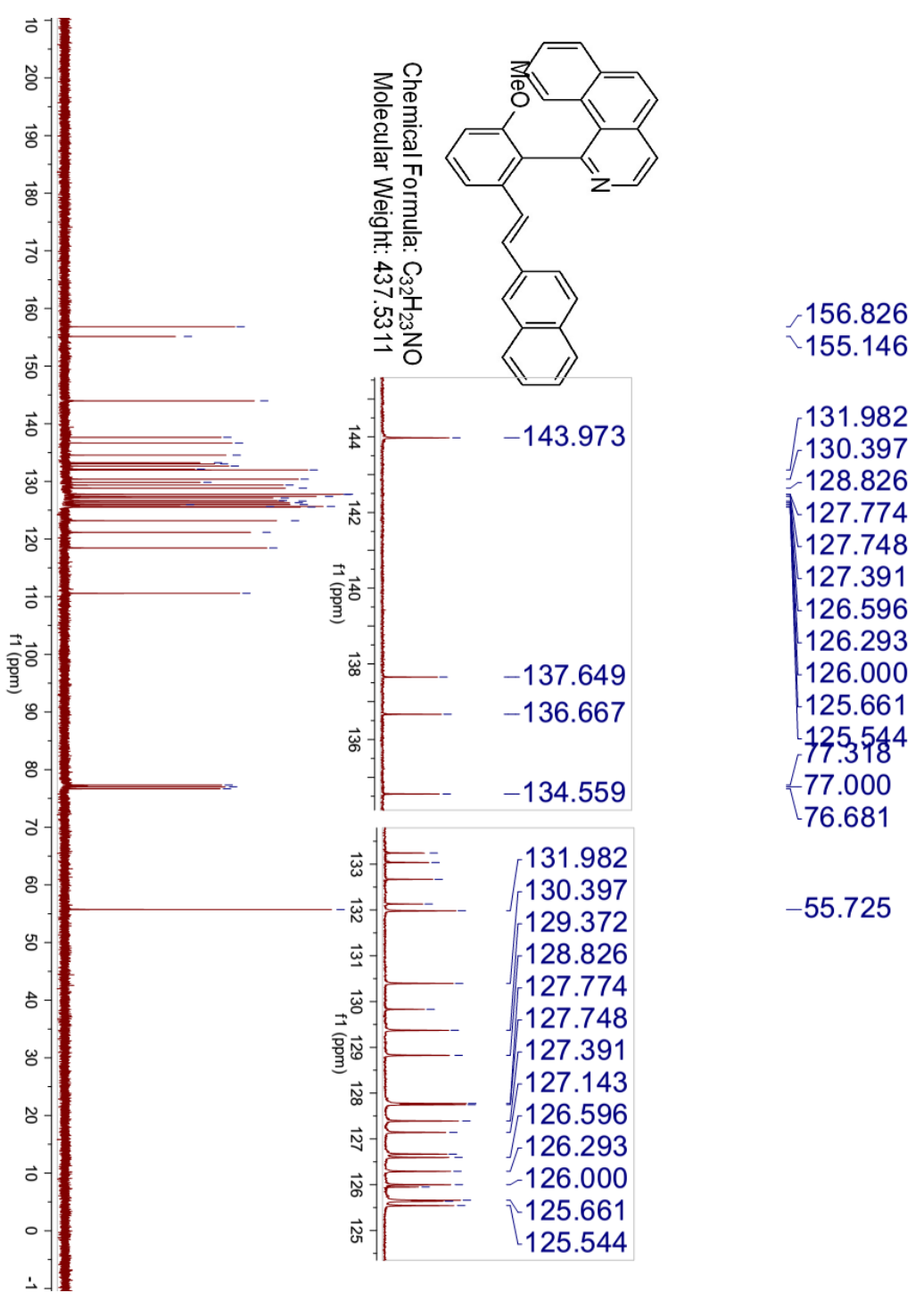




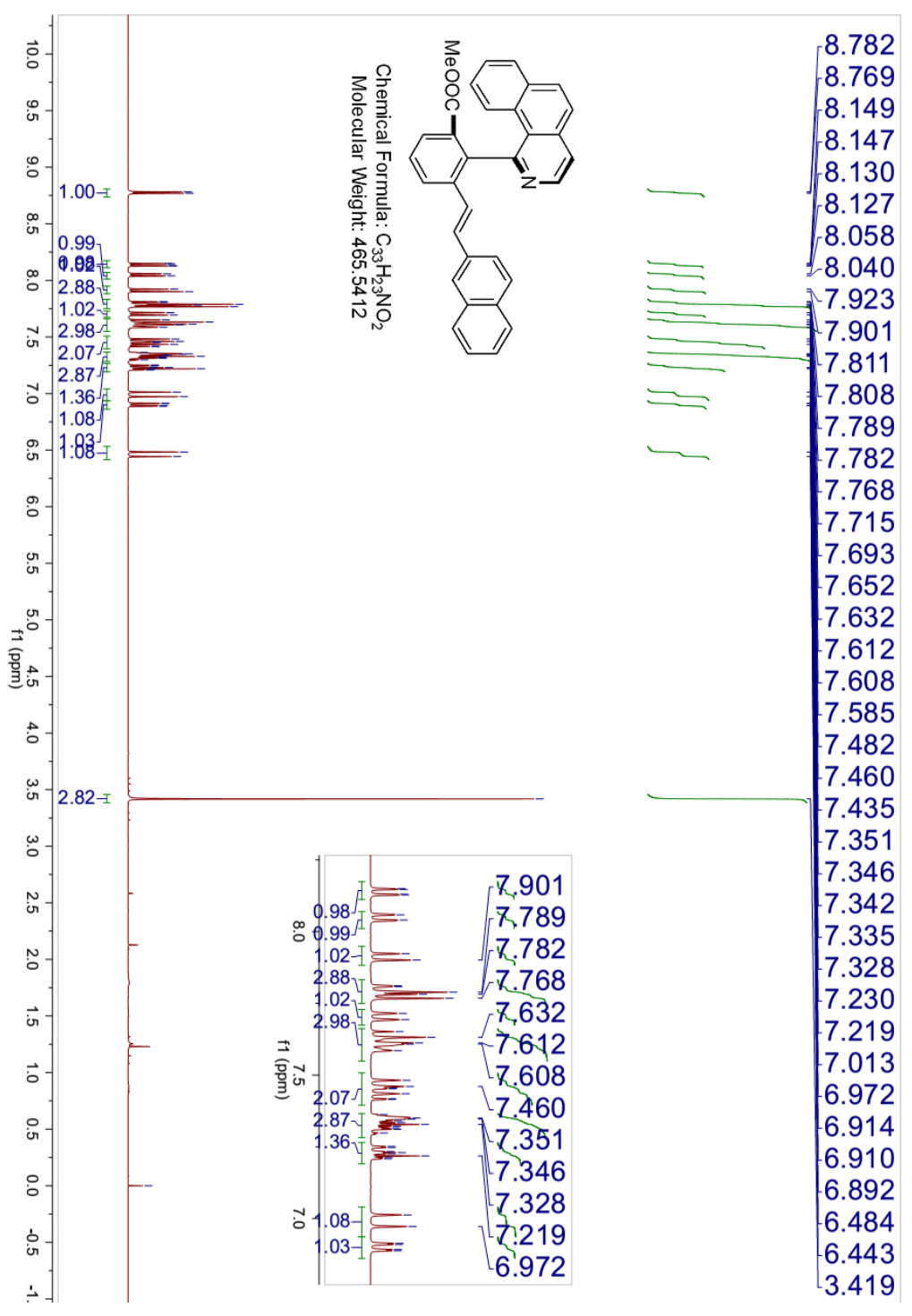




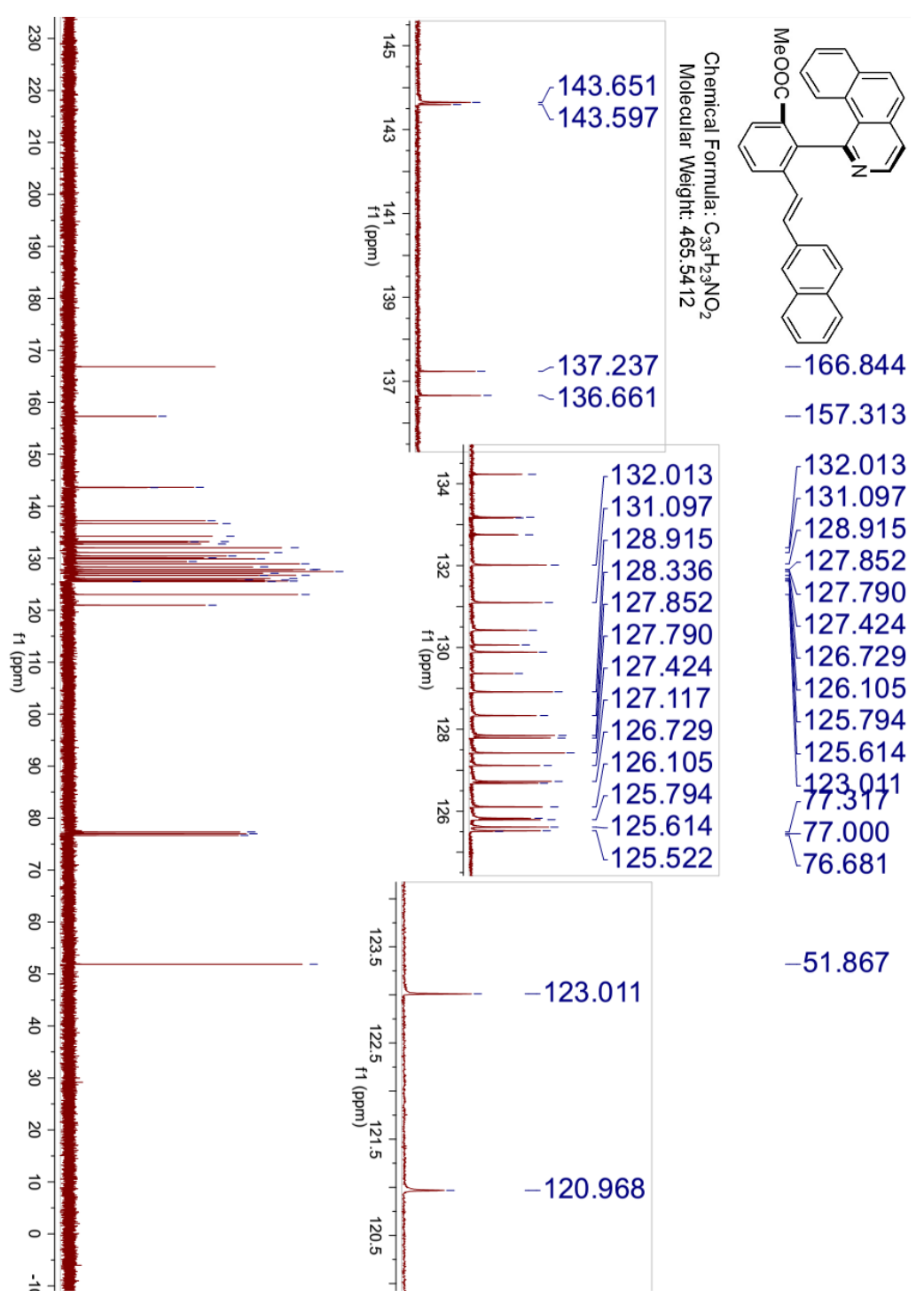




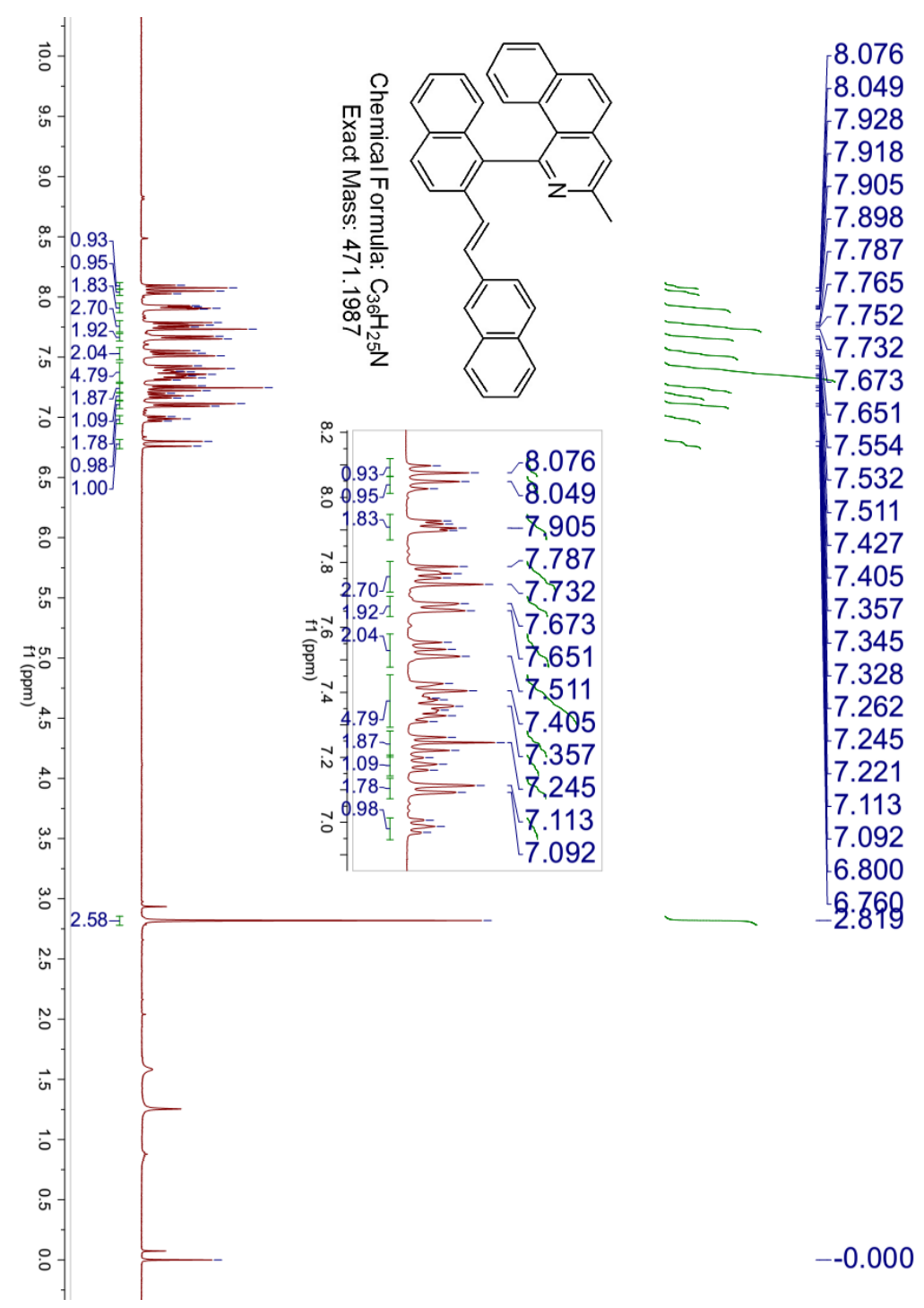



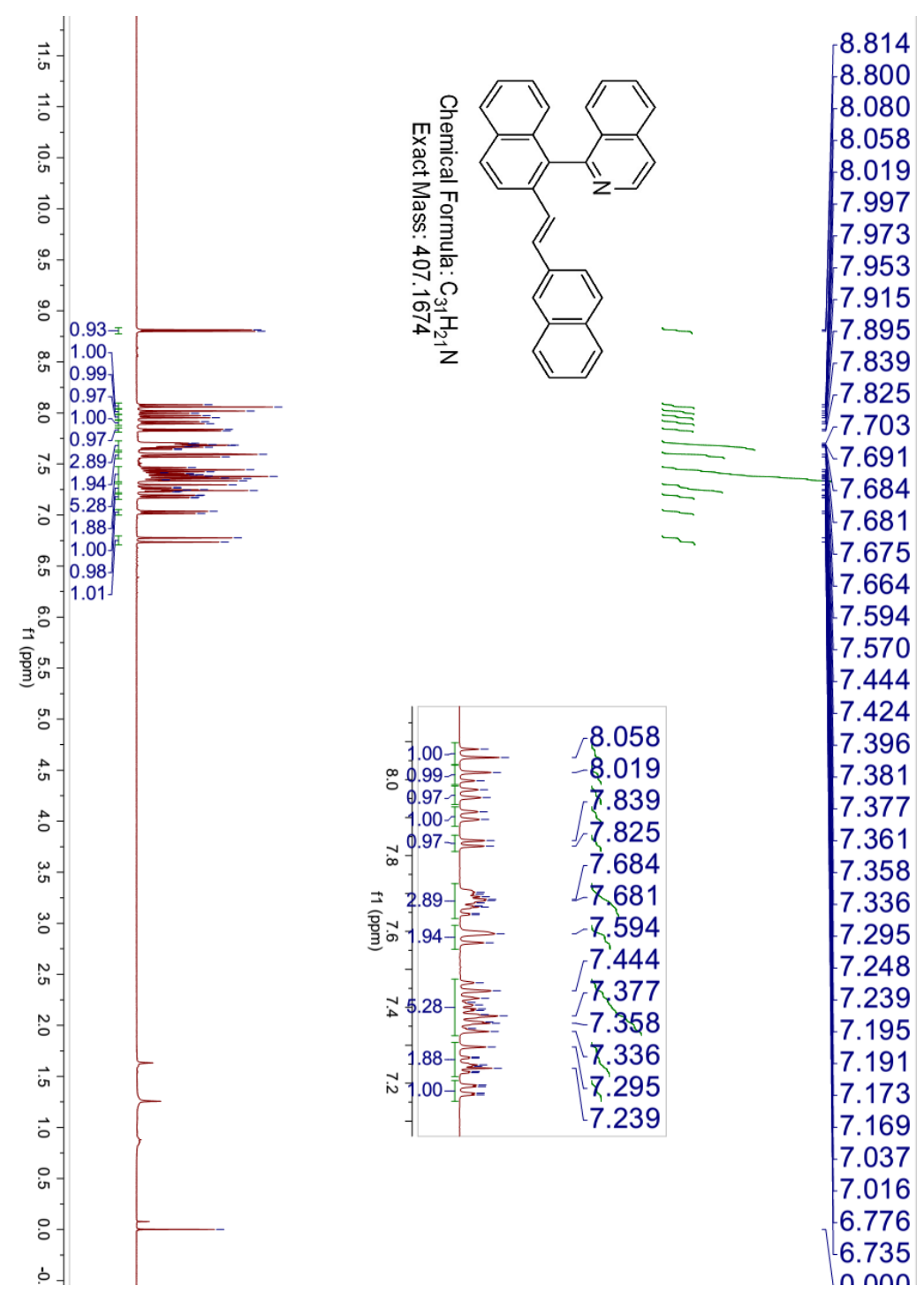


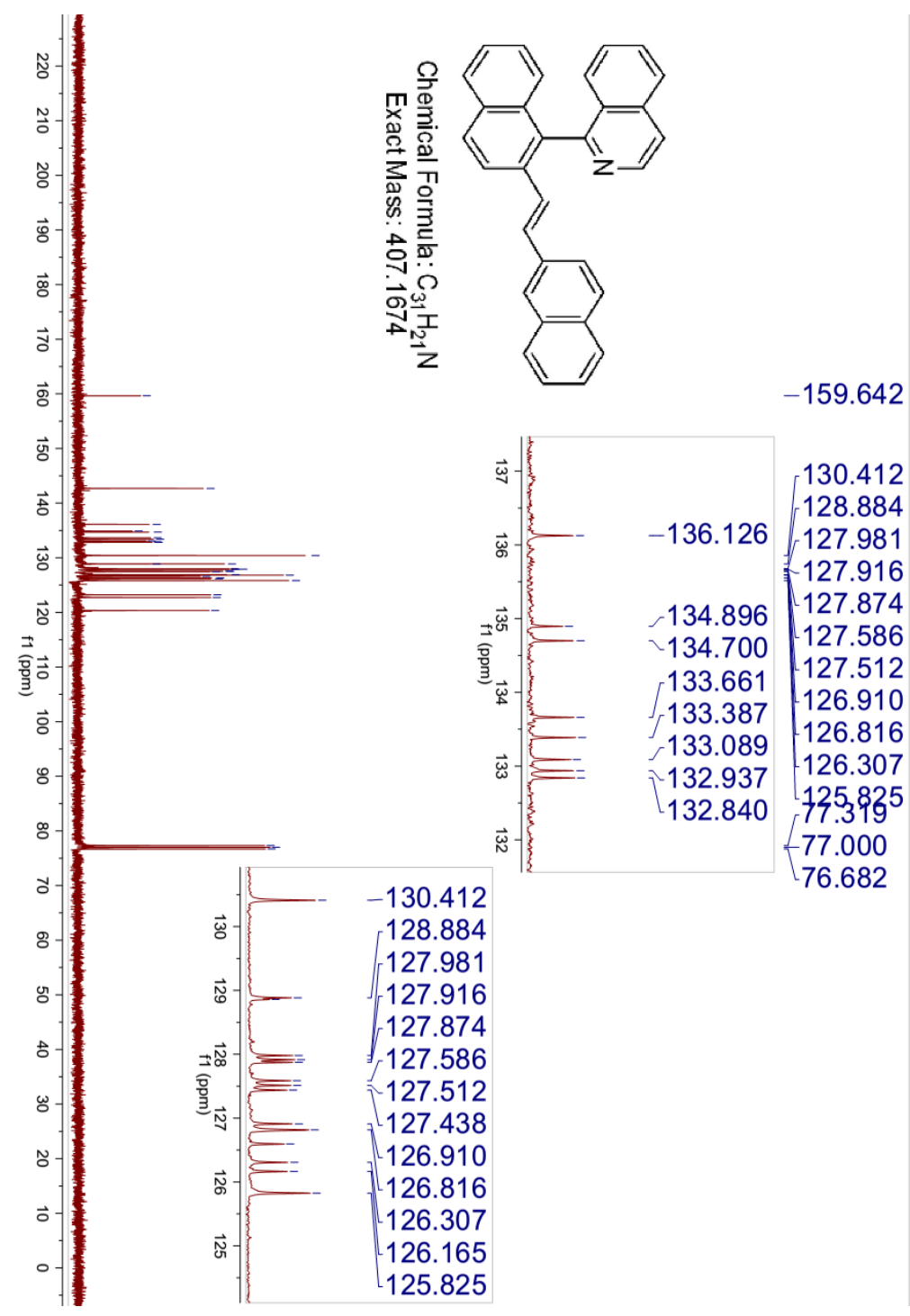




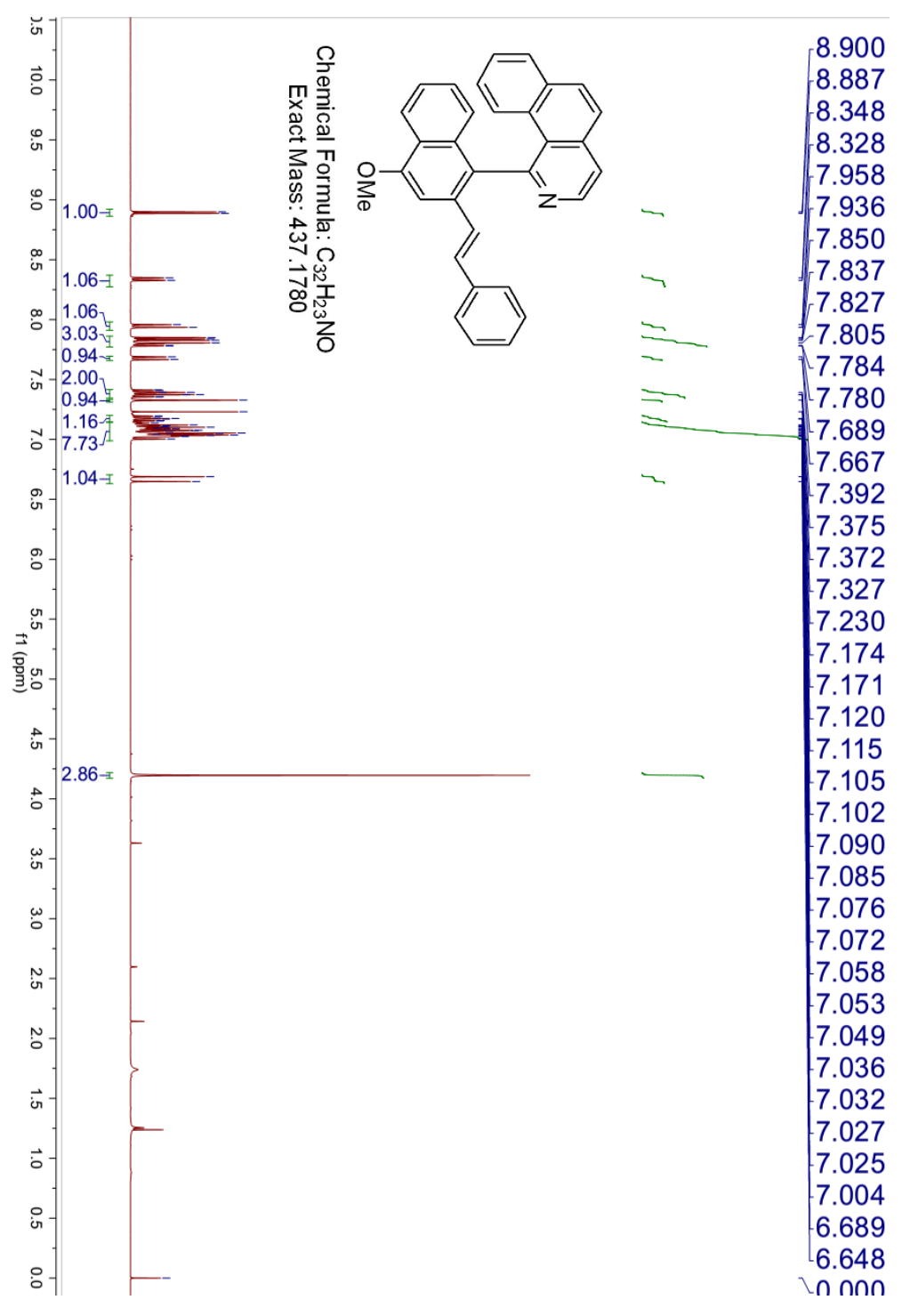




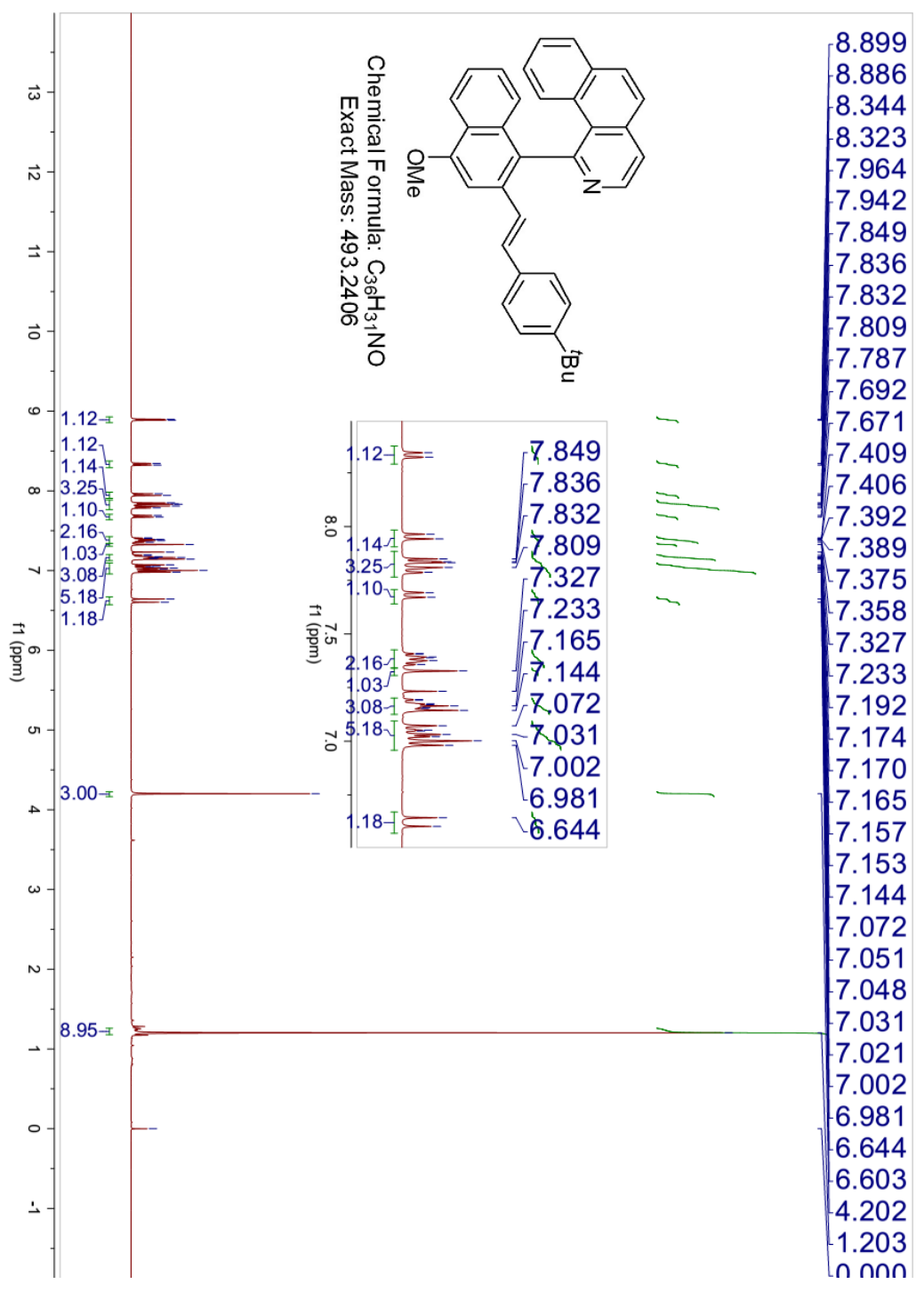




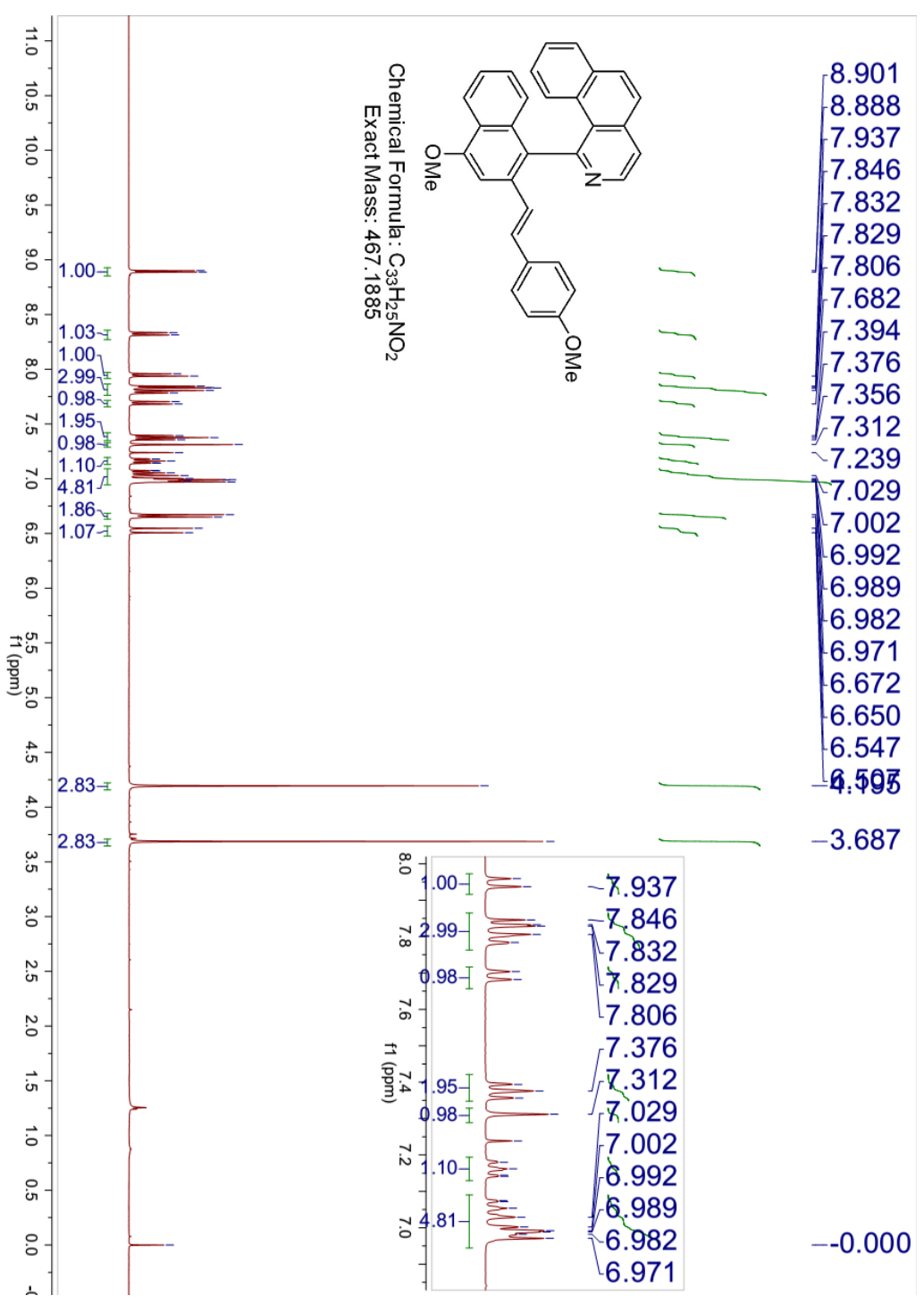




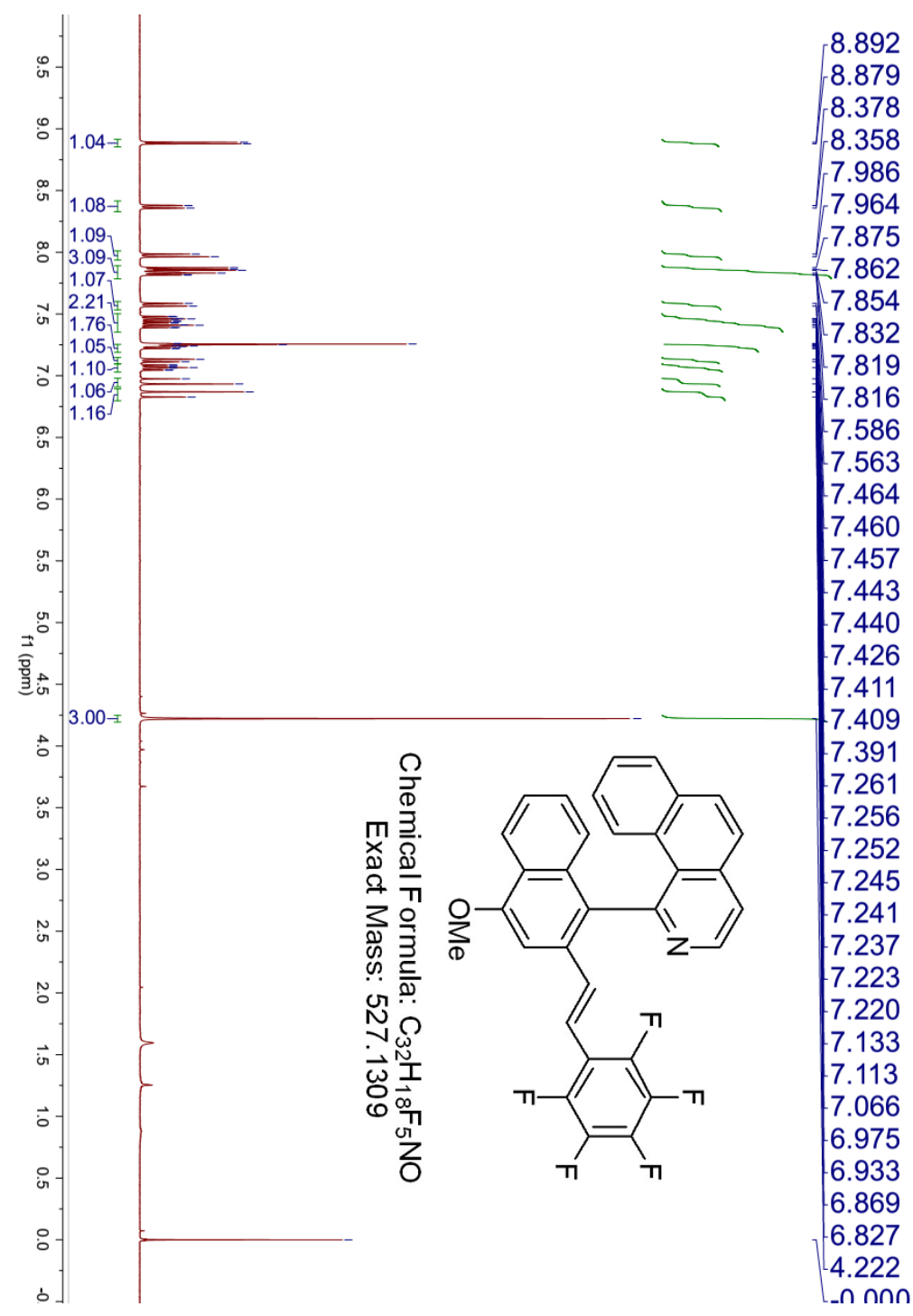



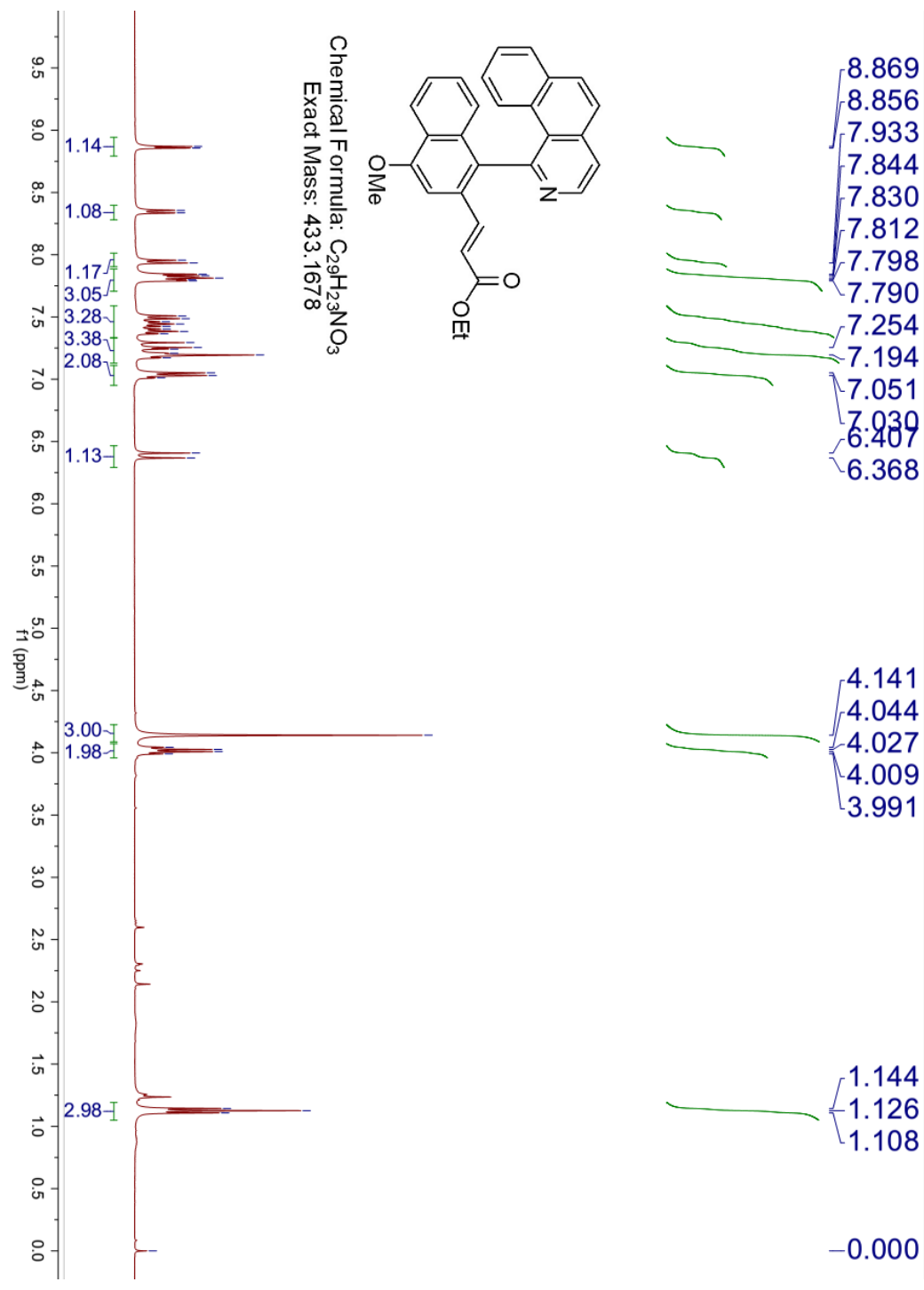


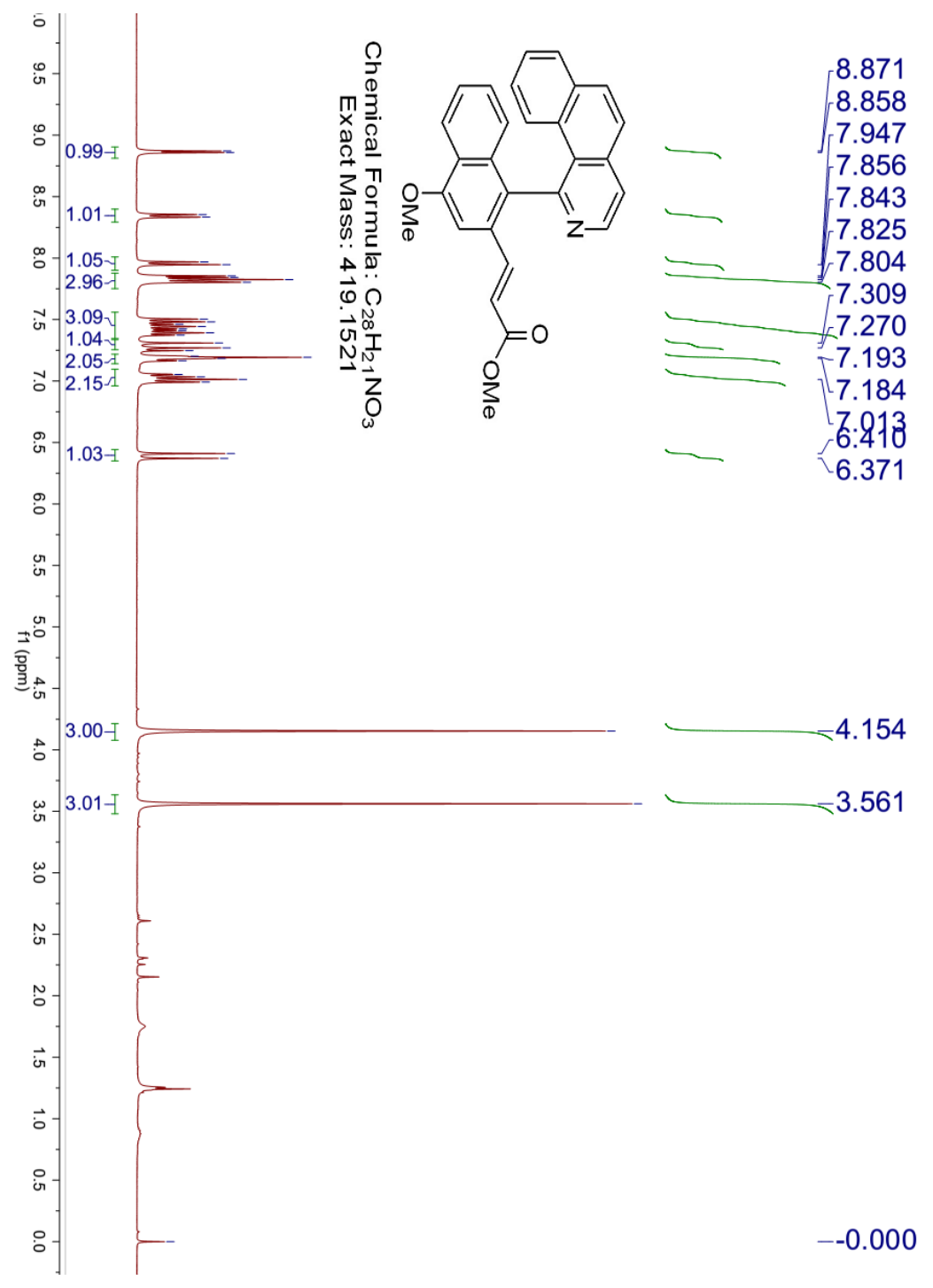




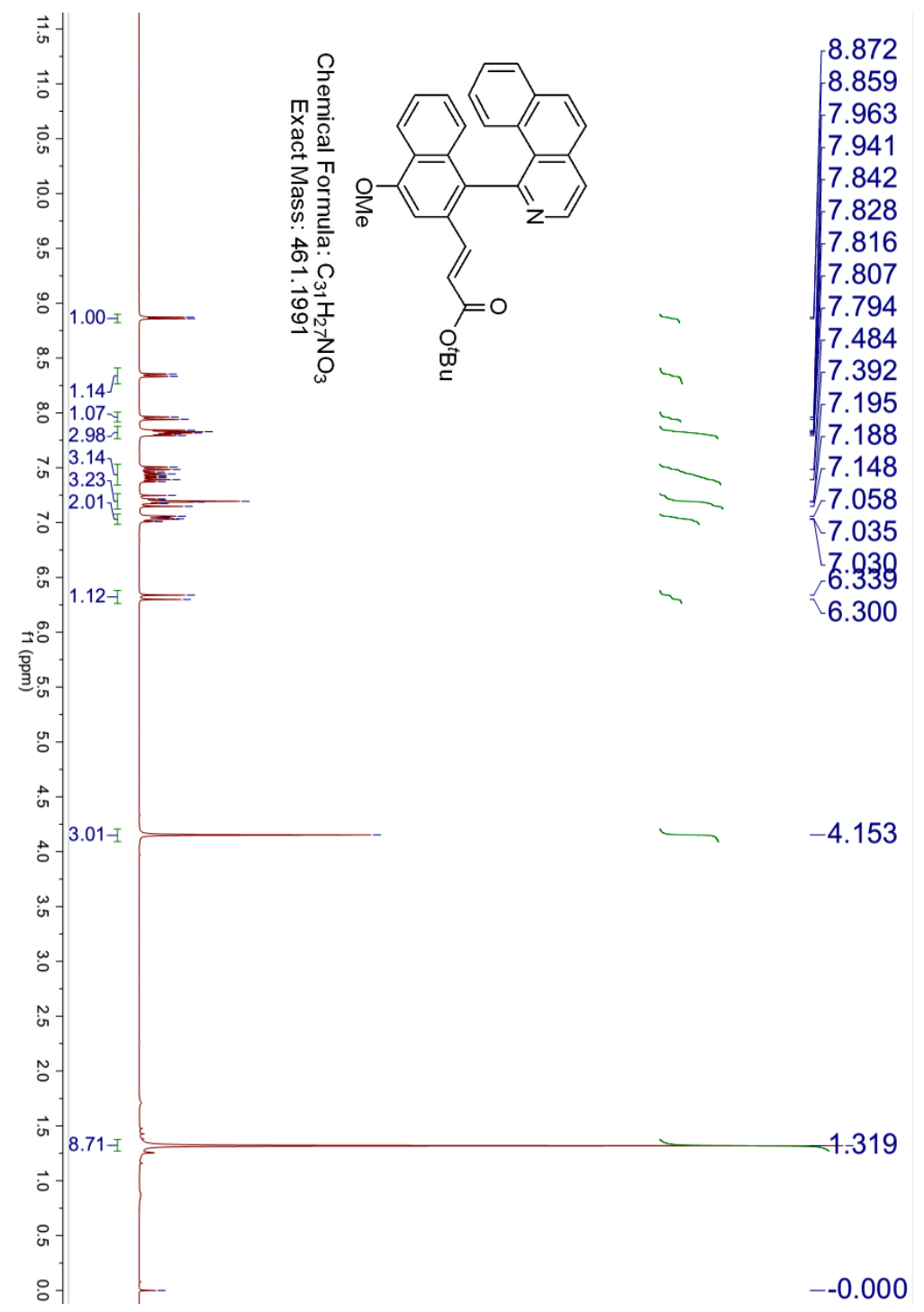




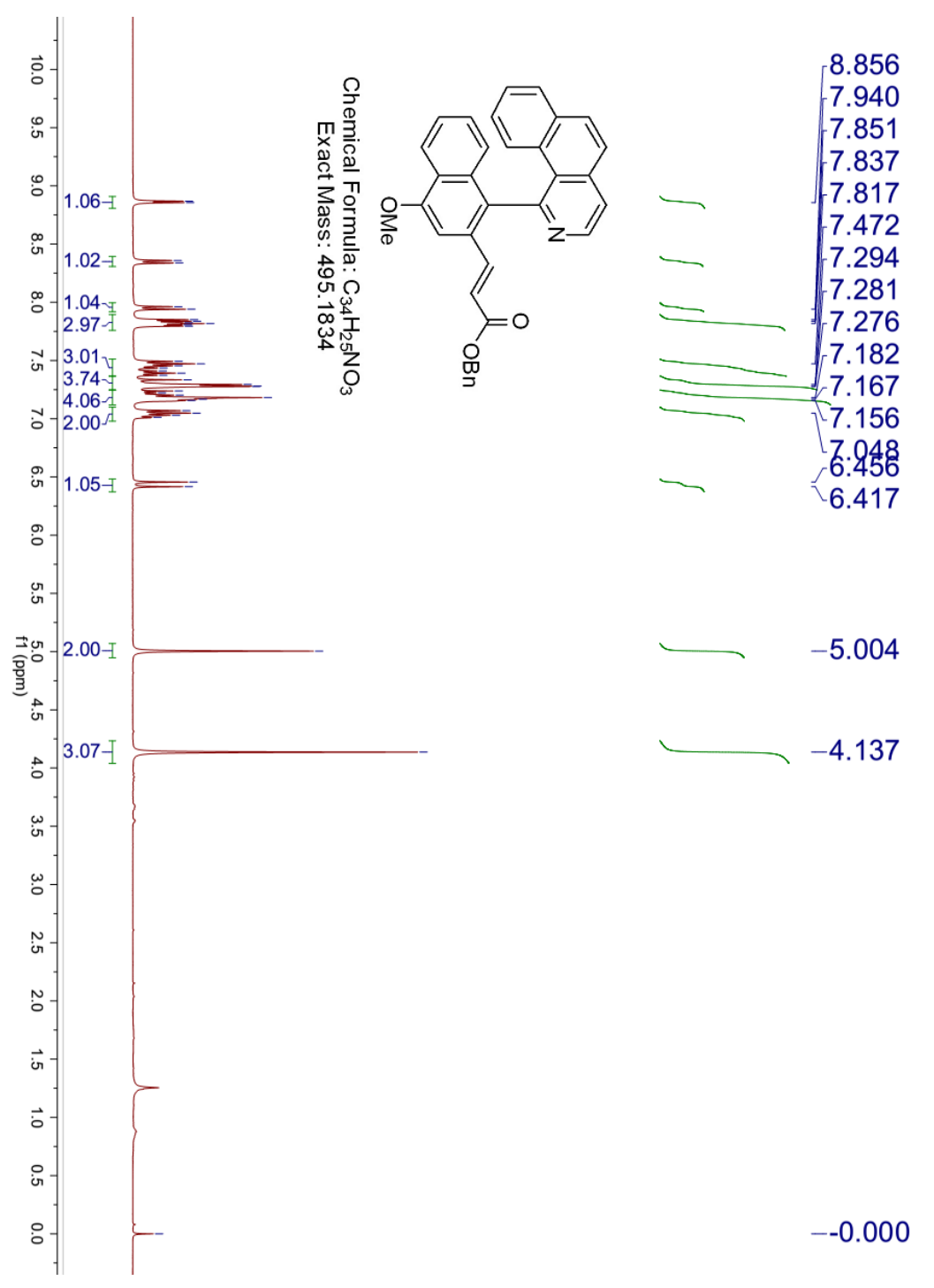




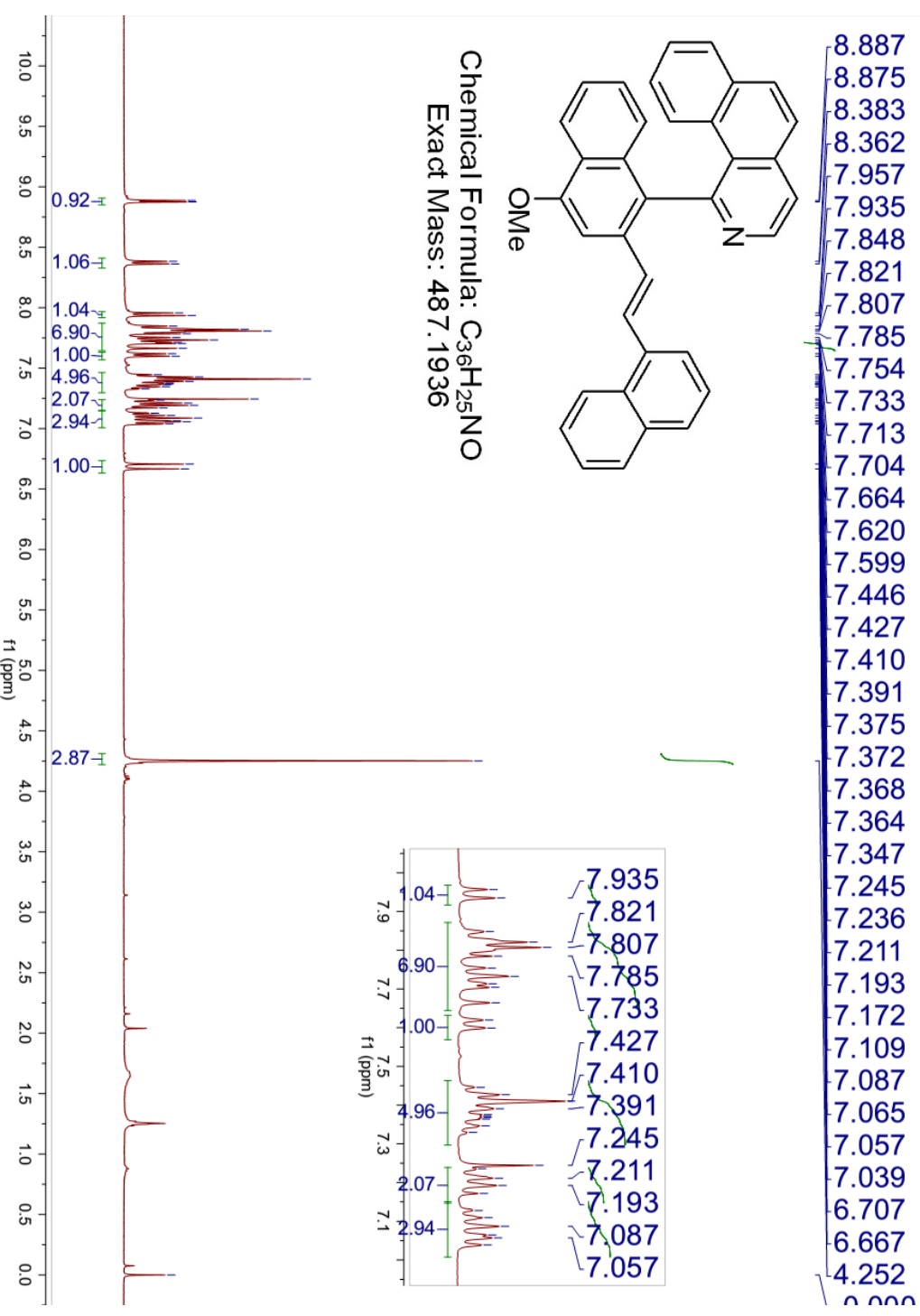



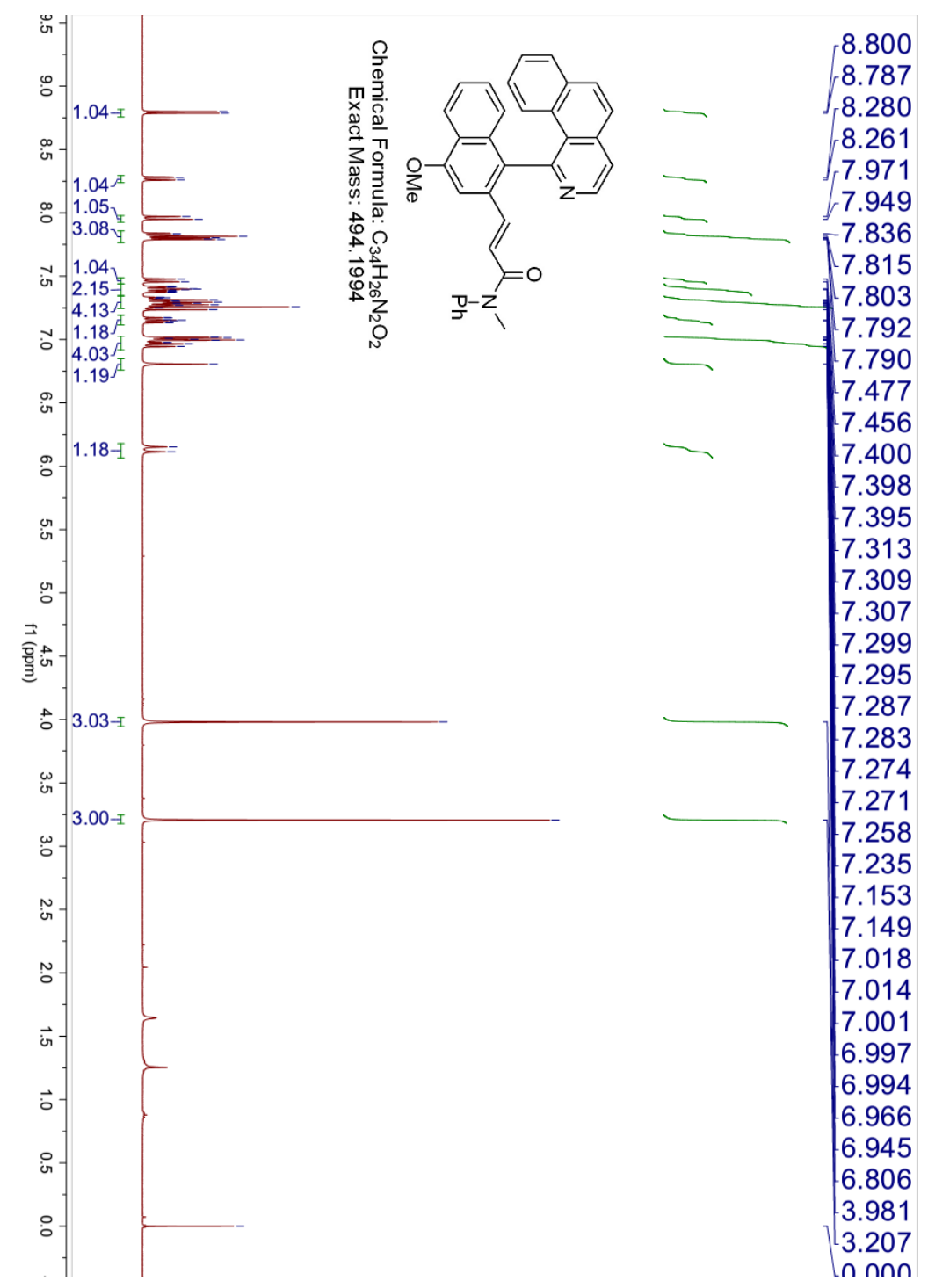


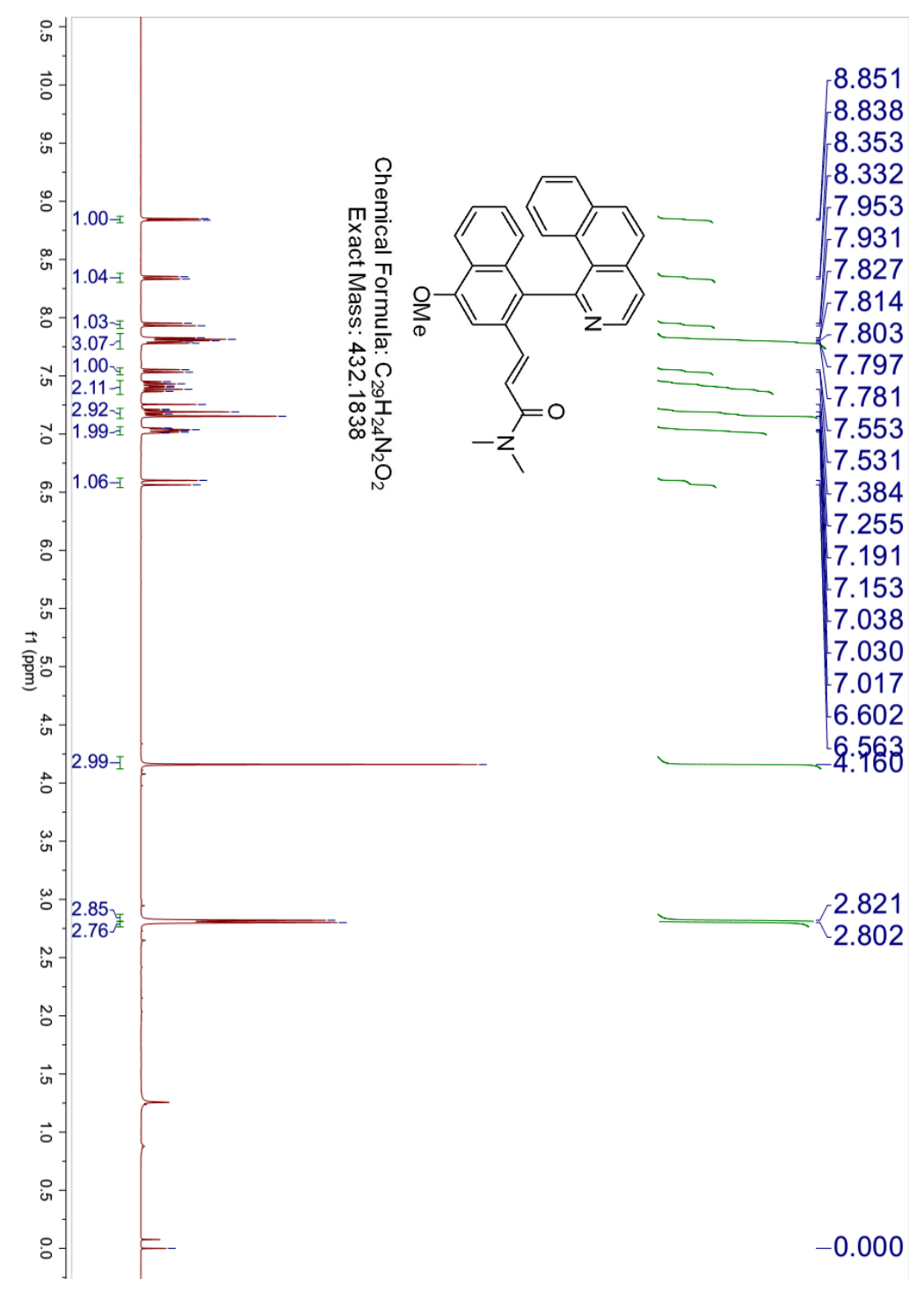




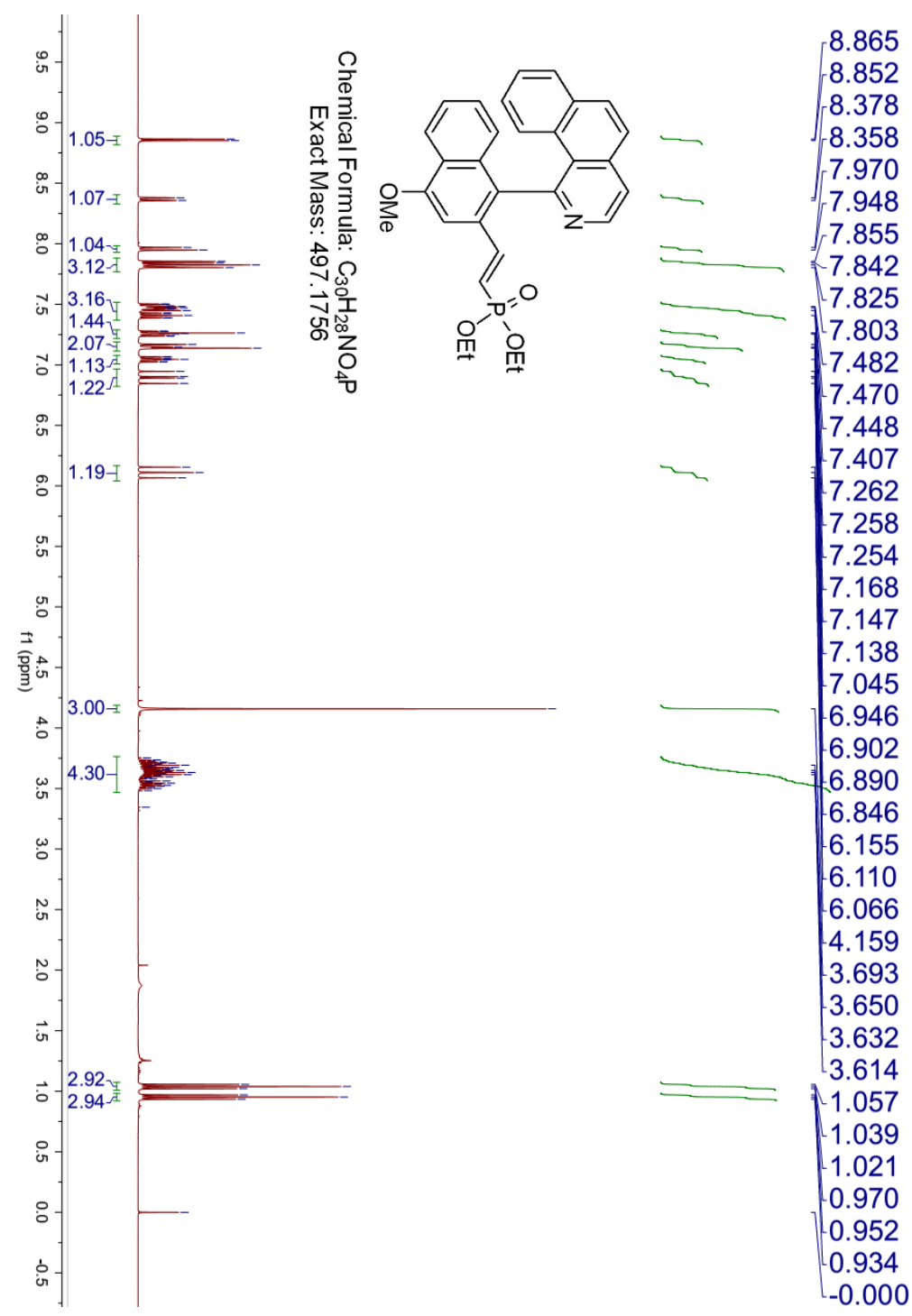




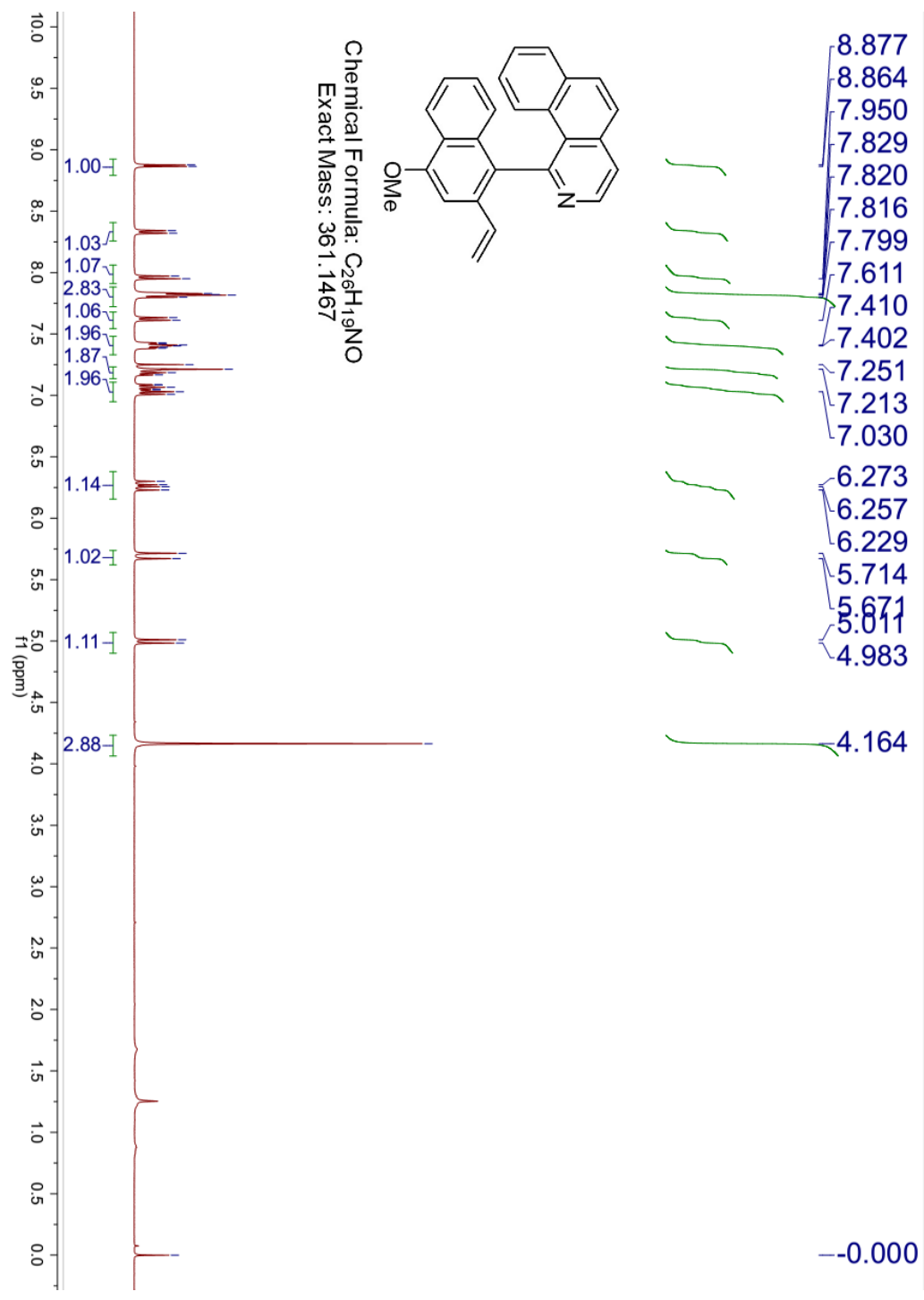




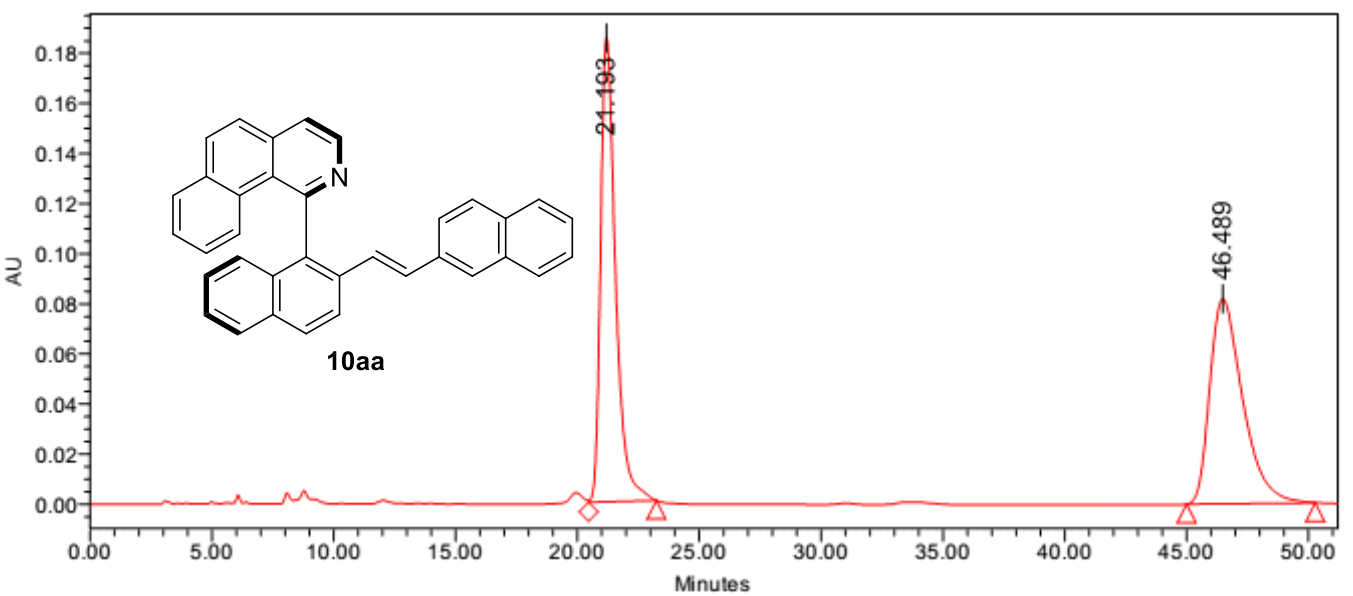

Peak Results

\begin{tabular}{|c|c|c|c|c|c|}
\hline & Name & RT & Area & Height & \% Area \\
\hline 1 & & 21.193 & 7873335 & 185316 & 50.66 \\
\hline 2 & & 46.489 & 7668350 & 81836 & 49.34 \\
\hline
\end{tabular}

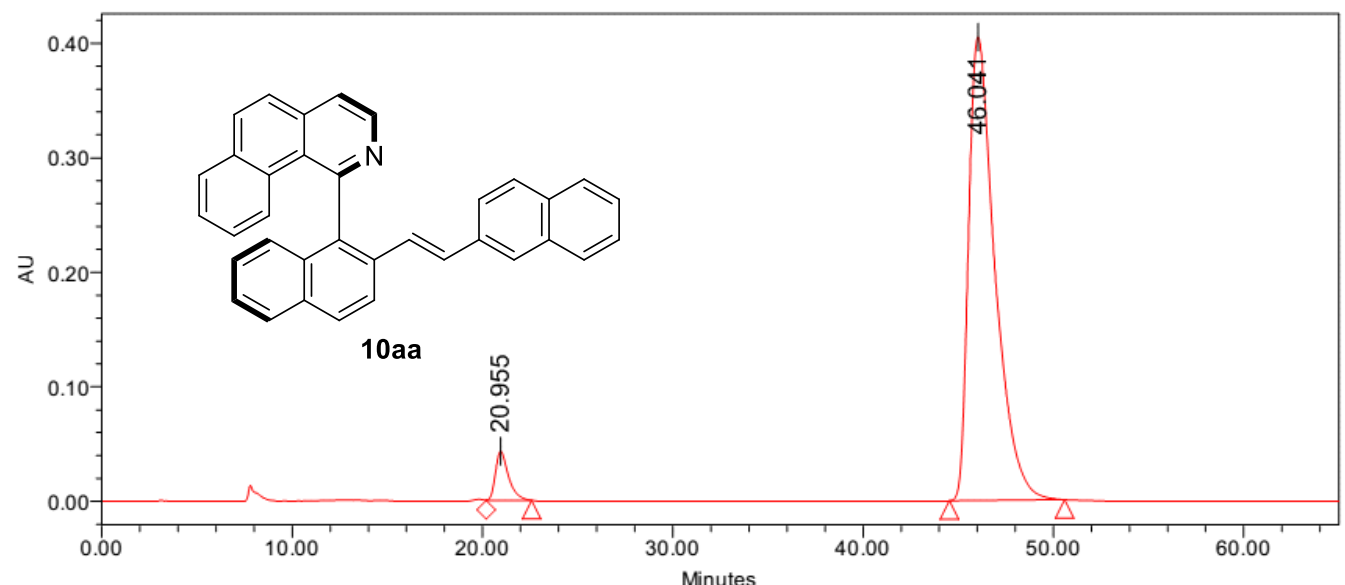

Peak Results

\begin{tabular}{|r|c|c|r|r|r|}
\hline & Name & RT & Area & Height & $\%$ Area \\
\hline 1 & & 20.955 & 2052975 & 42939 & 5.01 \\
\hline 2 & & 46.041 & 38934311 & 404563 & 94.99 \\
\hline
\end{tabular}




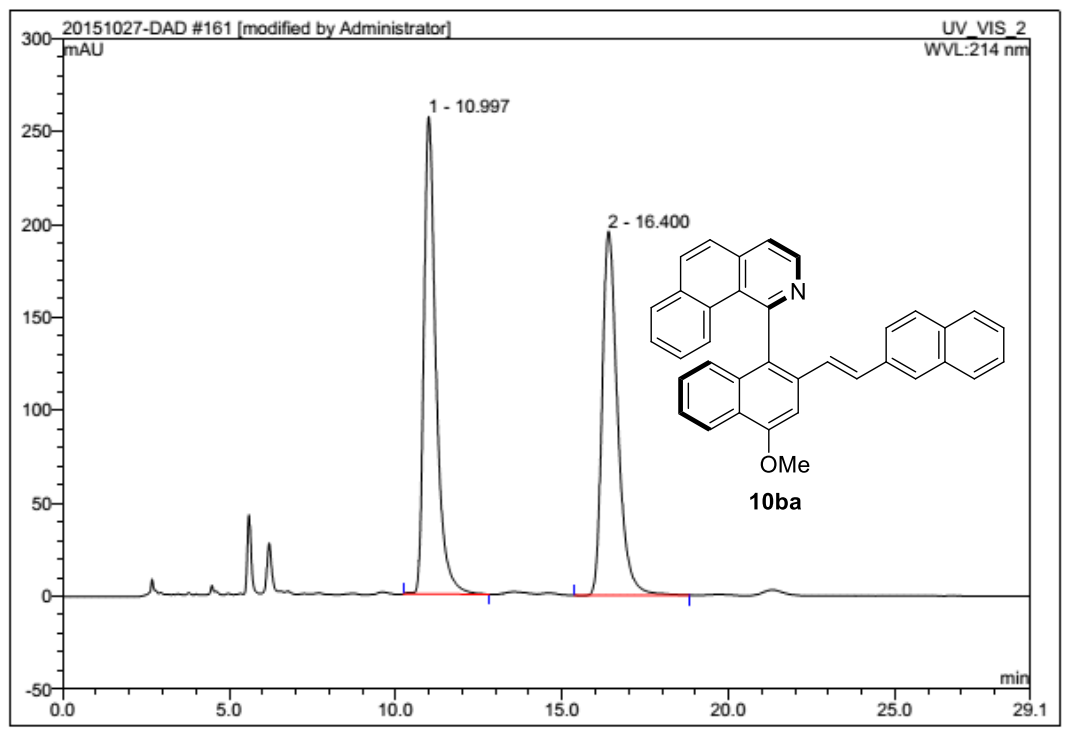

\begin{tabular}{|c|c|c|c|c|c|c|c|}
\hline No. & $\begin{array}{c}\text { Ret. Time } \\
\text { min }\end{array}$ & Peak Name & $\begin{array}{c}\text { Height } \\
\text { mAU }\end{array}$ & $\begin{array}{c}\text { Area } \\
\mathrm{mAU}^{*} \min \end{array}$ & $\begin{array}{c}\text { Rel.Area } \\
\%\end{array}$ & Amount & Type \\
\hline 1 & 11.00 & n.a. & 256.951 & 108.275 & 50.56 & n.a. & $\mathrm{BMB}^{*}$ \\
\hline 2 & 16.40 & n.a. & 195.842 & 105.893 & 49.44 & n.a. & $\mathrm{BMB}^{*}$ \\
\hline Total: & & & 452.792 & 214.167 & 100.00 & 0.000 & \\
\hline
\end{tabular}

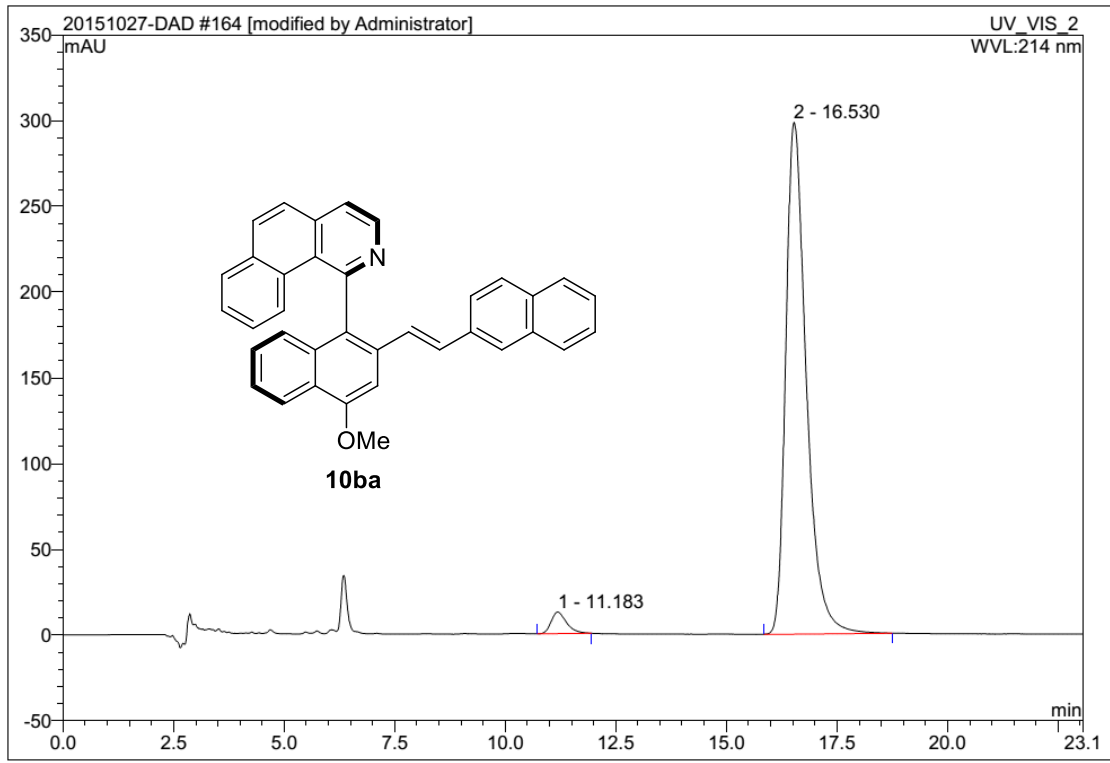

\begin{tabular}{|r|ccrrrrr|}
\hline No. & $\begin{array}{c}\text { Ret.Time } \\
\text { min }\end{array}$ & Peak Name & $\begin{array}{c}\text { Height } \\
\text { mAU }\end{array}$ & $\begin{array}{c}\text { Area } \\
\text { mAU*min }\end{array}$ & $\begin{array}{r}\text { Rel.Area } \\
\%\end{array}$ & Amount & Type \\
\hline 1 & 11.18 & n.a. & 12.575 & 5.155 & 3.11 & n.a. & BMB \\
2 & 16.53 & n.a. & 298.736 & 160.777 & 96.89 & n.a. & BMB \\
\hline Total: & & & 311.311 & 165.932 & 100.00 & 0.000 & \\
\hline
\end{tabular}




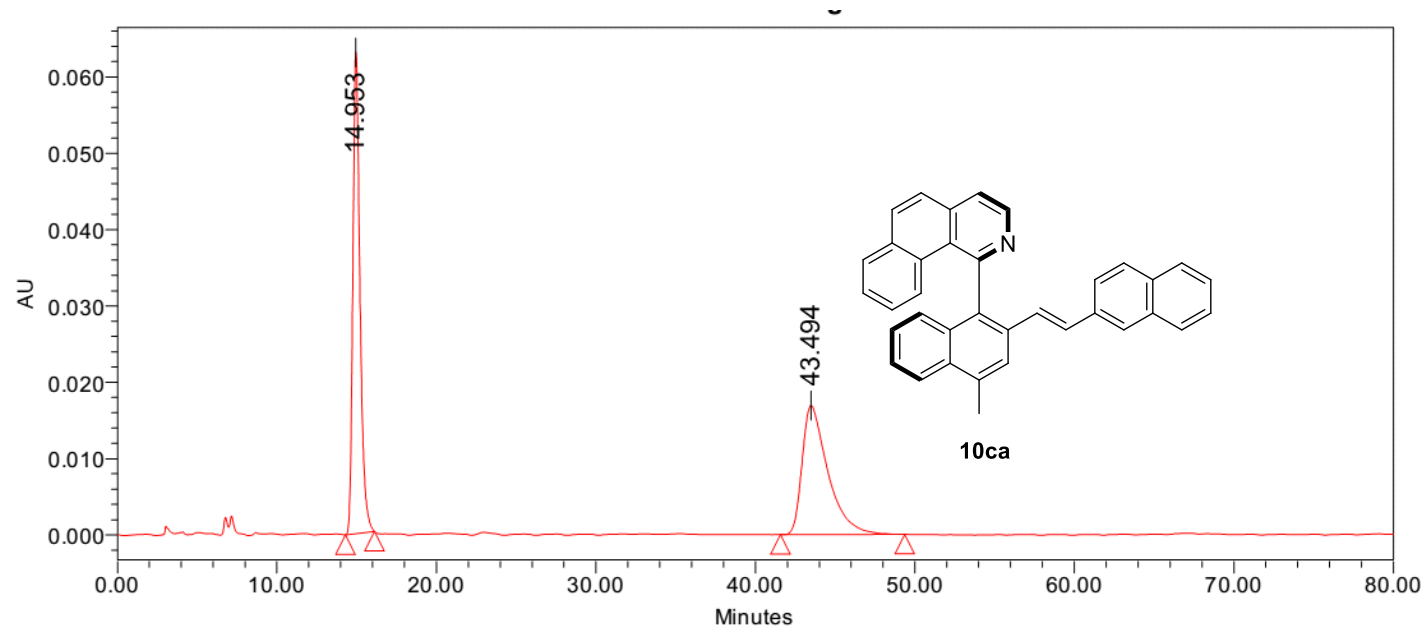

Peak Results

\begin{tabular}{|c|c|c|c|c|c|}
\hline \multicolumn{6}{|c|}{ Peak Results } \\
\hline & Name & RT & Area & Height & $\%$ Area \\
\hline 1 & & 14.953 & 1950108 & 63105 & 50.61 \\
\hline 2 & & 43.494 & 1903196 & 16960 & 49.39 \\
\hline
\end{tabular}

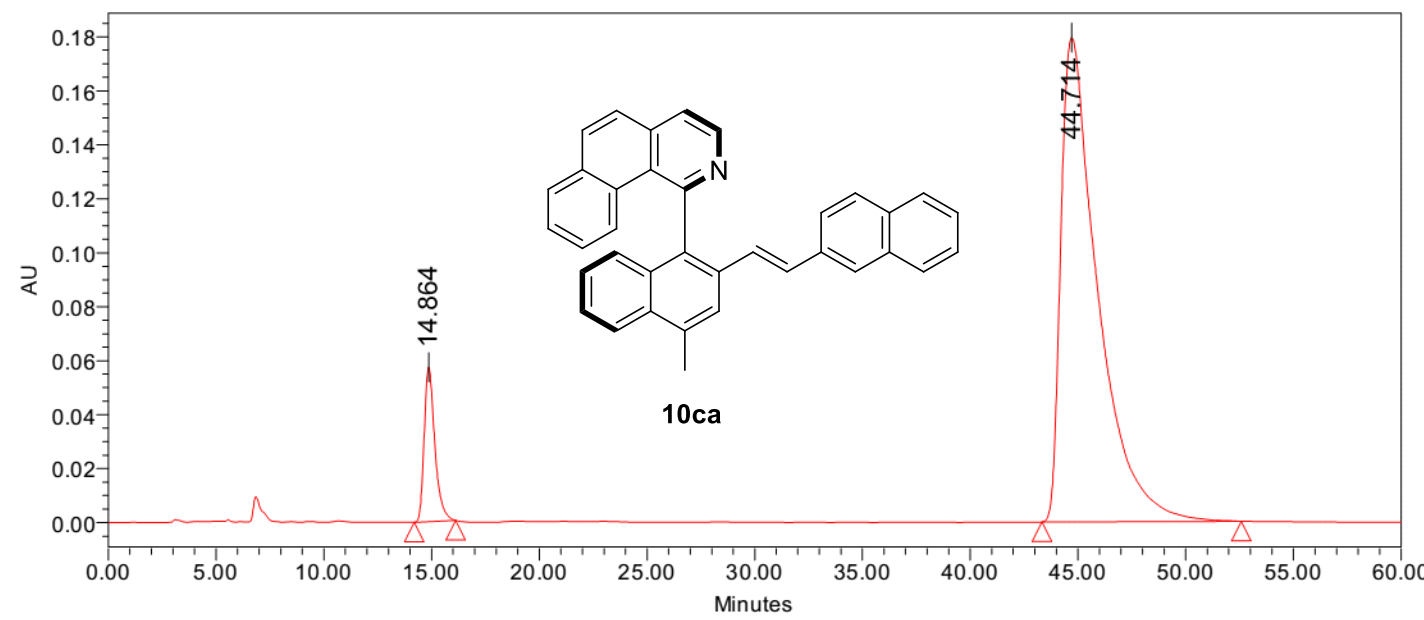

Peak Results

\begin{tabular}{|l|c|c|c|r|r|}
\hline & Name & RT & Area & Height & $\%$ Area \\
\hline 1 & & 14.864 & 1970637 & 57289 & 8.45 \\
\hline 2 & & 44.714 & 21344336 & 179435 & 91.55 \\
\hline
\end{tabular}




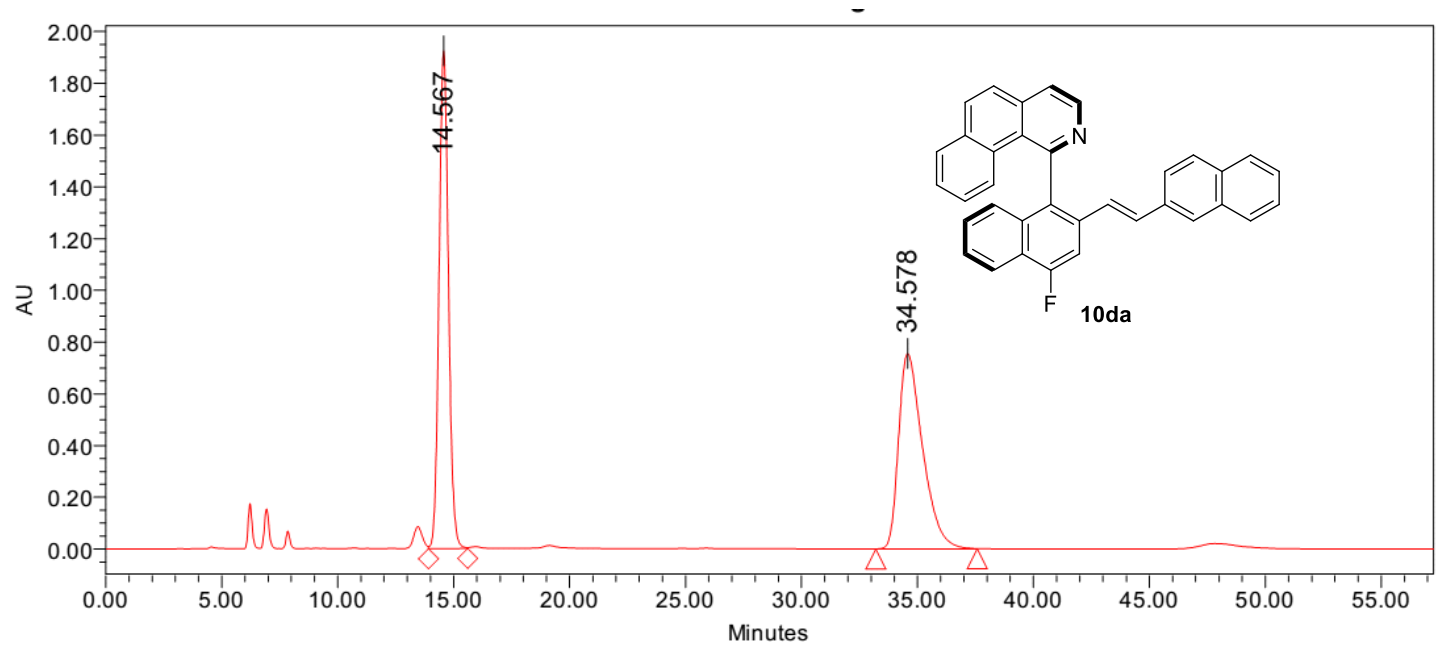

Peak Results

\begin{tabular}{|c|c|c|c|c|r|}
\hline & Name & RT & Area & Height & $\%$ Area \\
\hline 1 & & 14.567 & 54221223 & 1923135 & 49.96 \\
\hline 2 & & 34.578 & 54302052 & 755121 & 50.04 \\
\hline
\end{tabular}

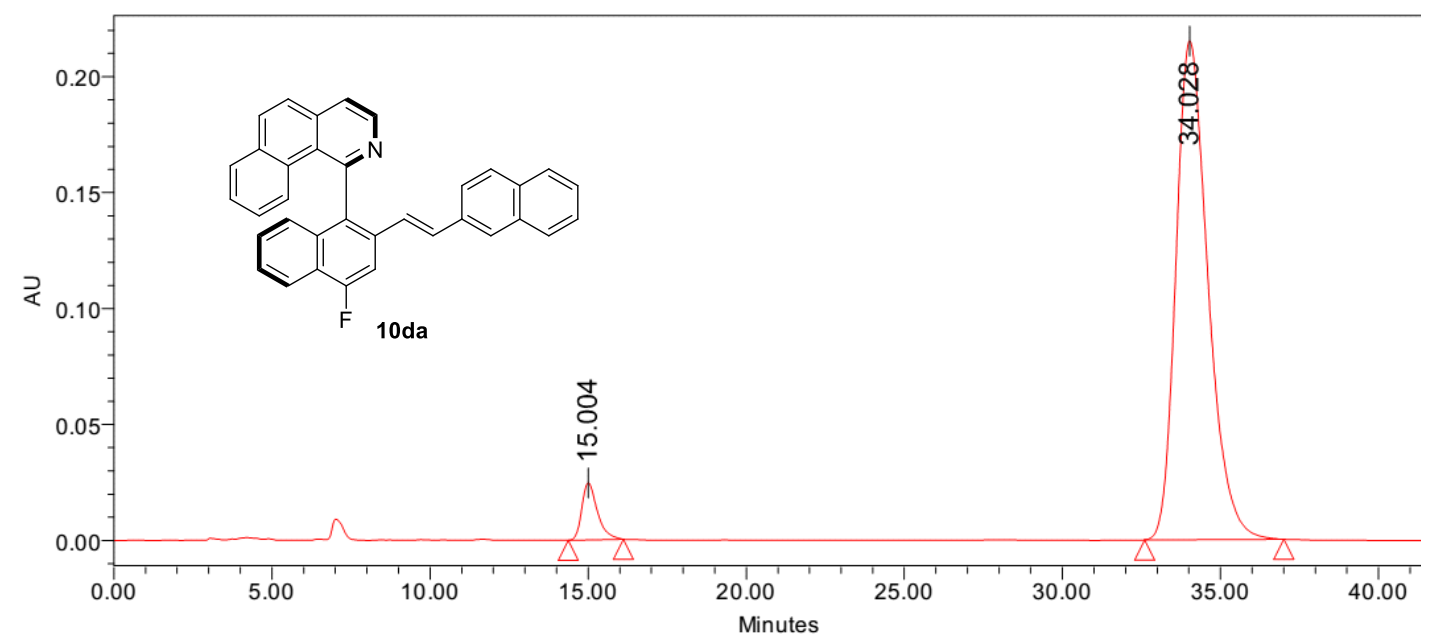

Peak Results

\begin{tabular}{|l|c|c|c|r|r|}
\hline & Name & RT & Area & Height & $\%$ Area \\
\hline 1 & & 15.004 & 842244 & 24607 & 5.26 \\
\hline 2 & & 34.028 & 15176626 & 215120 & 94.74 \\
\hline
\end{tabular}




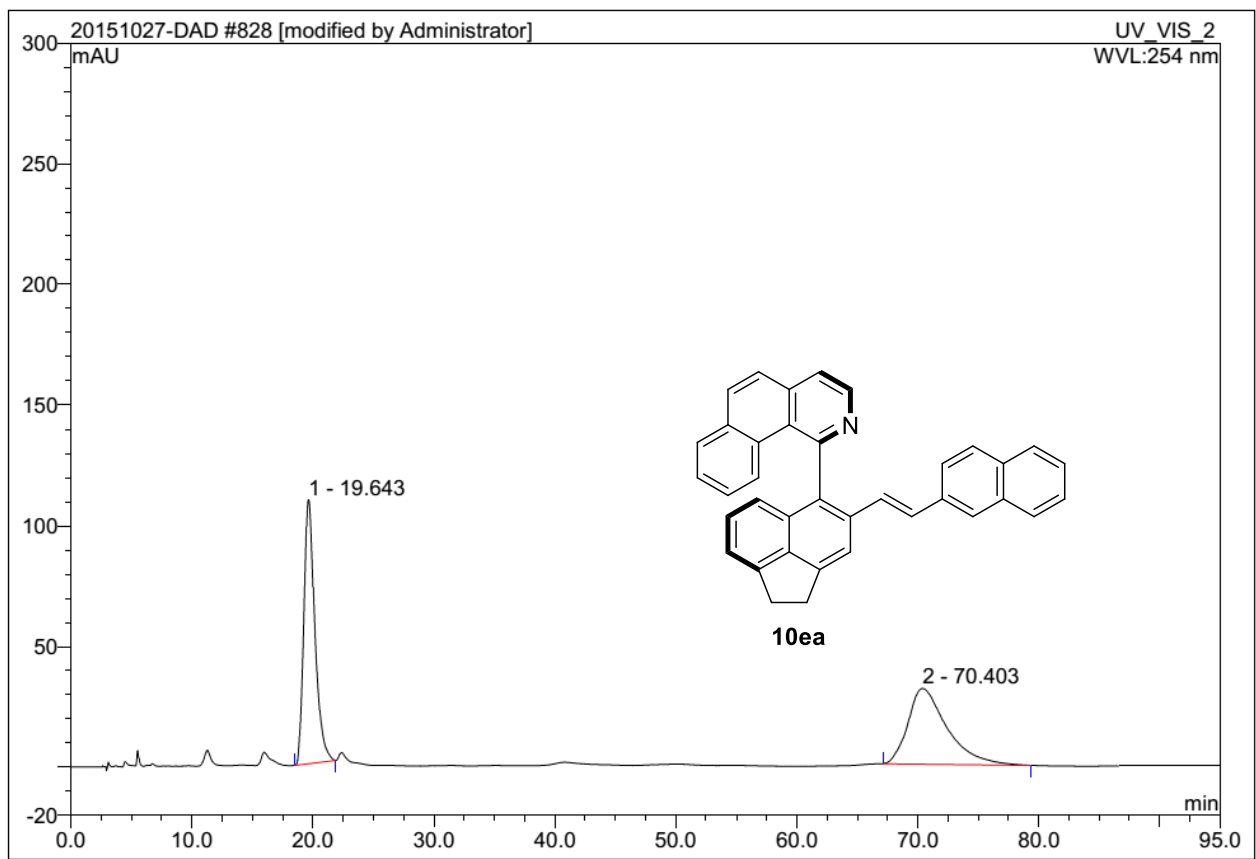

\begin{tabular}{|r|ccrrrrr|}
\hline No. & $\begin{array}{c}\text { Ret.Time } \\
\text { min }\end{array}$ & Peak Name & $\begin{array}{c}\text { Height } \\
\text { mAU }\end{array}$ & $\begin{array}{c}\text { Area } \\
\text { mAU*min }\end{array}$ & $\begin{array}{r}\text { Rel.Area } \\
\%\end{array}$ & Amount & Type \\
\hline 1 & 19.64 & n.a. & 109.544 & 116.095 & 49.76 & n.a. & BMB $^{*}$ \\
2 & 70.40 & n.a. & 31.338 & 117.218 & 50.24 & n.a. & BMB $^{*}$ \\
\hline Total: & & & 140.882 & 233.313 & 100.00 & 0.000 & \\
\hline
\end{tabular}

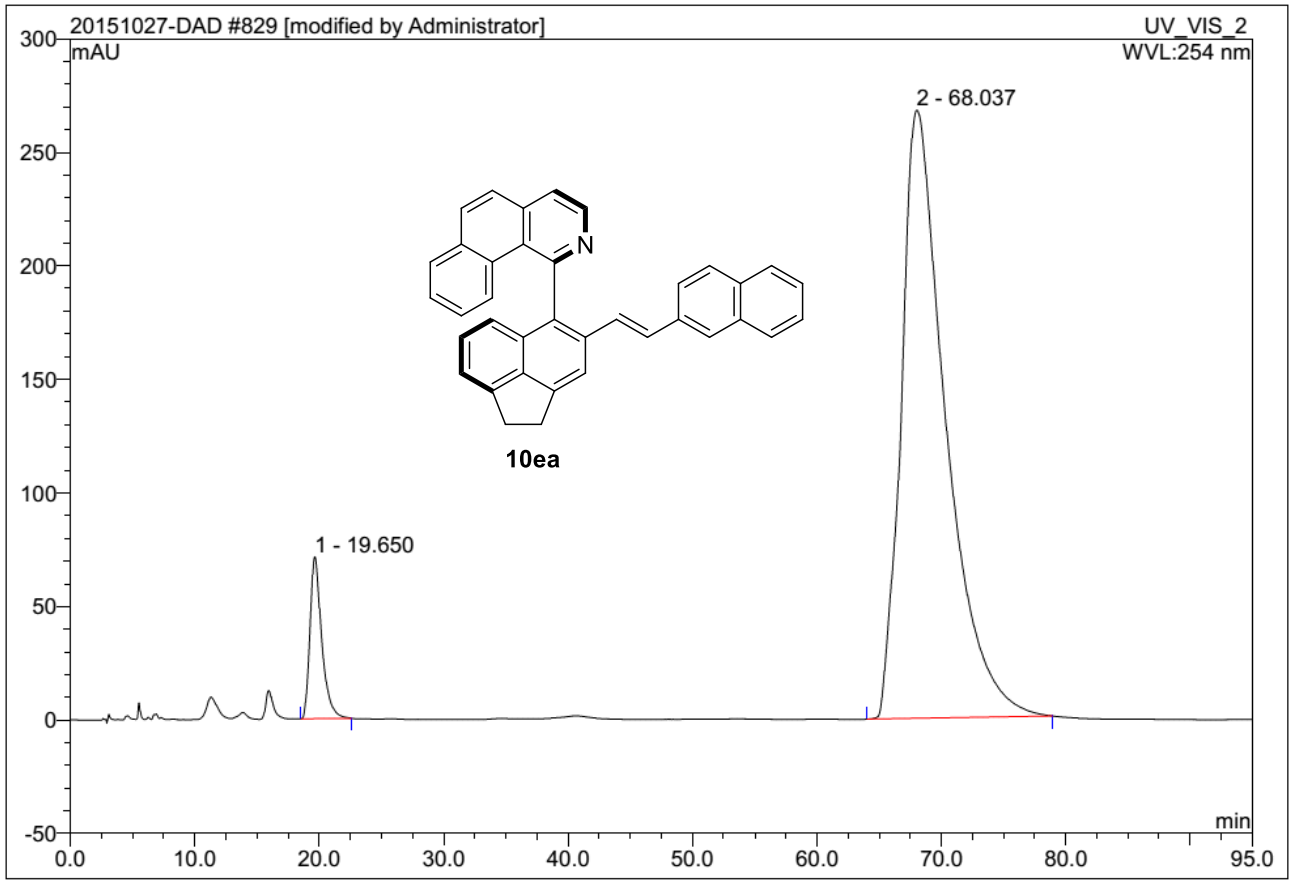

\begin{tabular}{|r|ccrrrrr|}
\hline No. & $\begin{array}{c}\text { Ret.Time } \\
\text { min }\end{array}$ & Peak Name & $\begin{array}{c}\text { Height } \\
\text { mAU }\end{array}$ & $\begin{array}{c}\text { Area } \\
\text { mAU*min }\end{array}$ & $\begin{array}{r}\text { Rel.Area } \\
\%\end{array}$ & Amount & Type \\
\hline 1 & 19.65 & n.a. & 71.353 & 78.198 & 6.62 & n.a. & BMB \\
2 & 68.04 & n.a. & 268.003 & 1103.905 & 93.38 & n.a. & BMB \\
\hline Total: & & & 339.356 & 1182.103 & 100.00 & 0.000 & \\
\hline
\end{tabular}




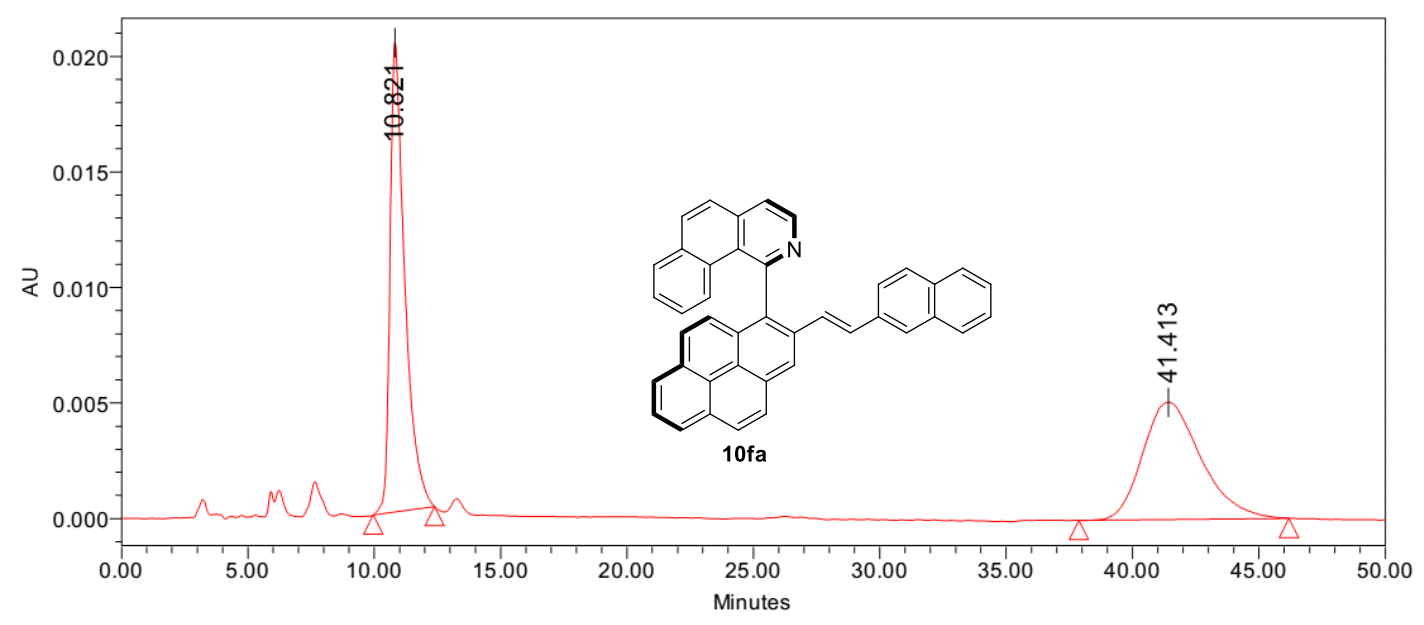

Peak Results

\begin{tabular}{|c|c|c|c|c|c|}
\hline \multicolumn{6}{|c|}{ Peak Results } \\
\hline & Name & RT & Area & Height & $\%$ Area \\
\hline 1 & & 10.821 & 826907 & 20312 & 50.39 \\
\hline 2 & & 41.413 & 813964 & 5070 & 49.61 \\
\hline
\end{tabular}

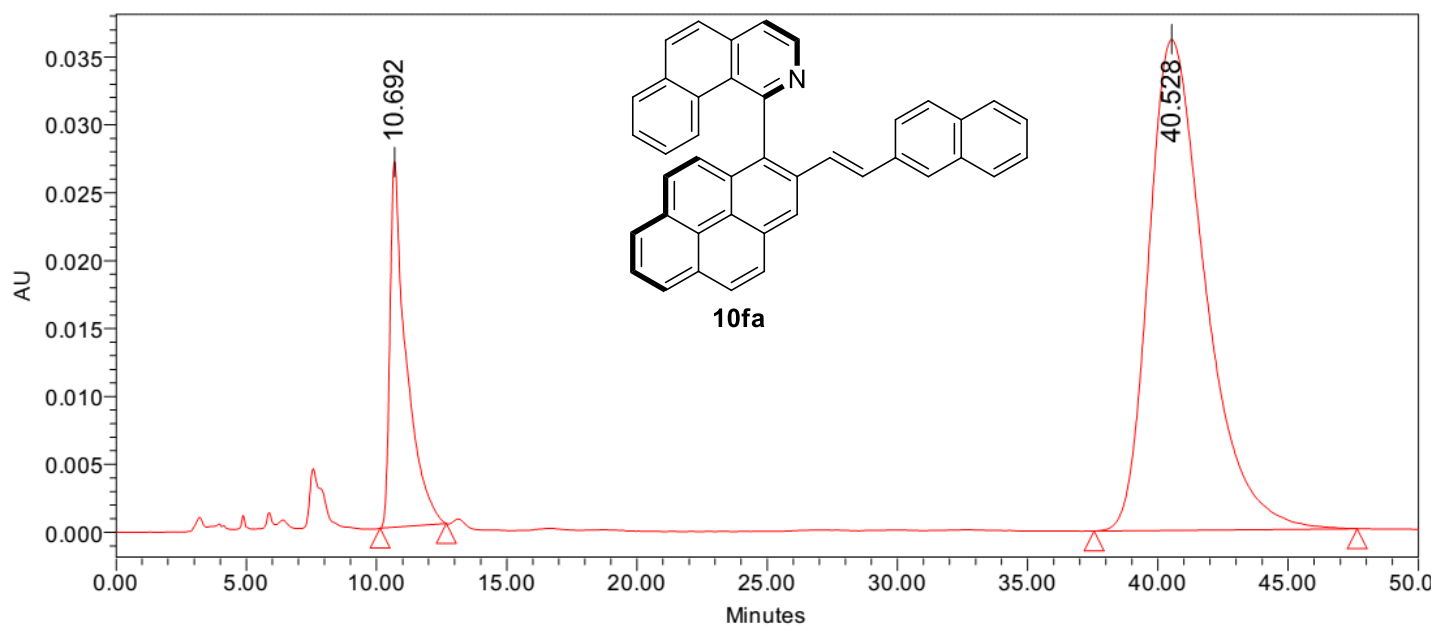

Peak Results

\begin{tabular}{|c|c|c|c|c|r|}
\hline & Name & RT & Area & Height & $\%$ Area \\
\hline 1 & & 10.692 & 1172398 & 26928 & 17.67 \\
\hline 2 & & 40.528 & 5463161 & 36149 & 82.33 \\
\hline
\end{tabular}




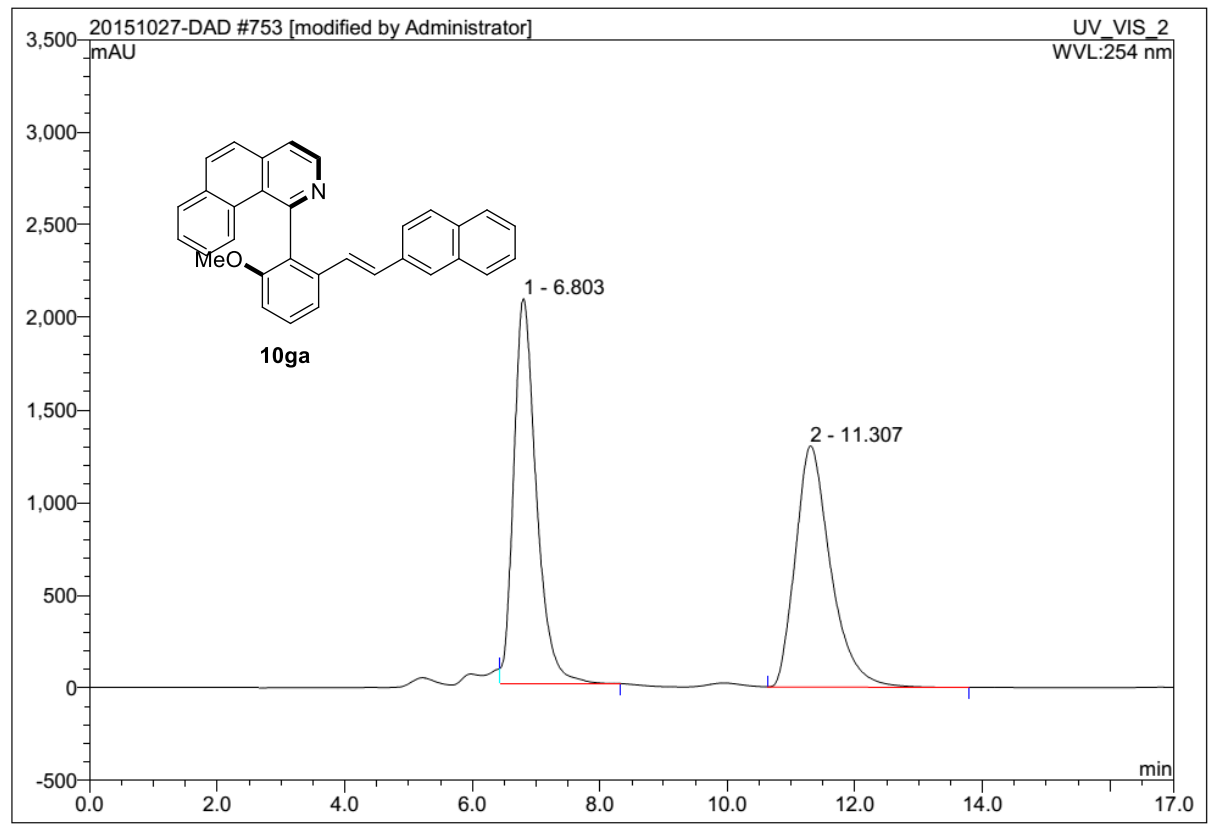

\begin{tabular}{|r|rrrrrrr|}
\hline No. & $\begin{array}{c}\text { Ret.Time } \\
\text { min }\end{array}$ & Peak Name & $\begin{array}{c}\text { Height } \\
\text { mAU }\end{array}$ & $\begin{array}{c}\text { Area } \\
\text { mAU*min }\end{array}$ & $\begin{array}{c}\text { Rel.Area } \\
\%\end{array}$ & Amount & Type \\
\hline 1 & 6.80 & n.a. & 2078.075 & 852.839 & 50.51 & n.a. & MB $^{*}$ \\
2 & 11.31 & n.a. & 1302.442 & 835.645 & 49.49 & n.a. & BMB $^{*}$ \\
\hline Total: & & & 3380.517 & 1688.483 & 100.00 & 0.000 & \\
\hline
\end{tabular}

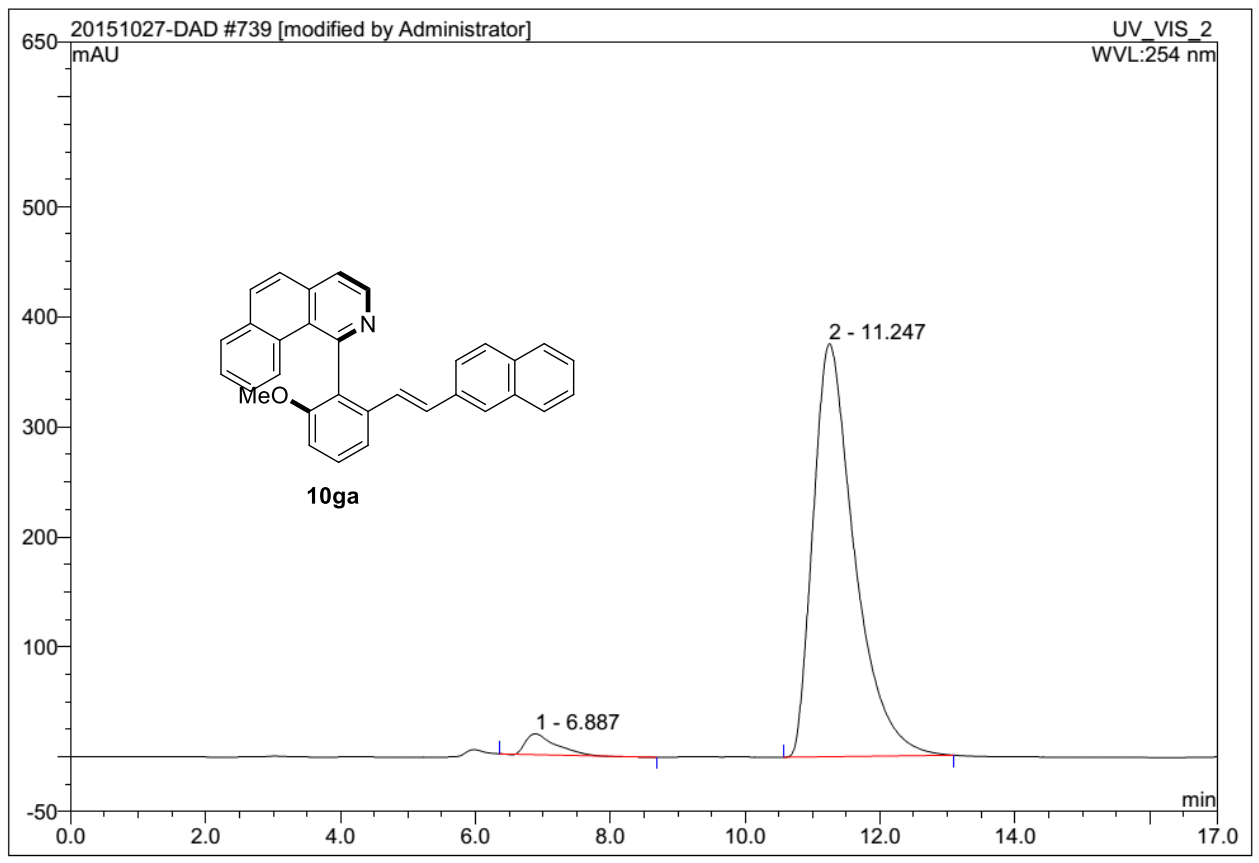

\begin{tabular}{|r|rrrrrrr|}
\hline No. & $\begin{array}{c}\text { Ret.Time } \\
\text { min }\end{array}$ & Peak Name & $\begin{array}{c}\text { Height } \\
\text { mAU }\end{array}$ & $\begin{array}{r}\text { Area } \\
\text { mAU*min }\end{array}$ & $\begin{array}{r}\text { Rel.Area } \\
\%\end{array}$ & Amount & Type \\
\hline 1 & 6.89 & n.a. & 18.938 & 9.412 & 3.41 & n.a. & BMB $^{*}$ \\
2 & 11.25 & n.a. & 375.458 & 266.876 & 96.59 & n.a. & BMB \\
\hline Total: & & & 394.397 & 276.288 & 100.00 & 0.000 & \\
\hline
\end{tabular}




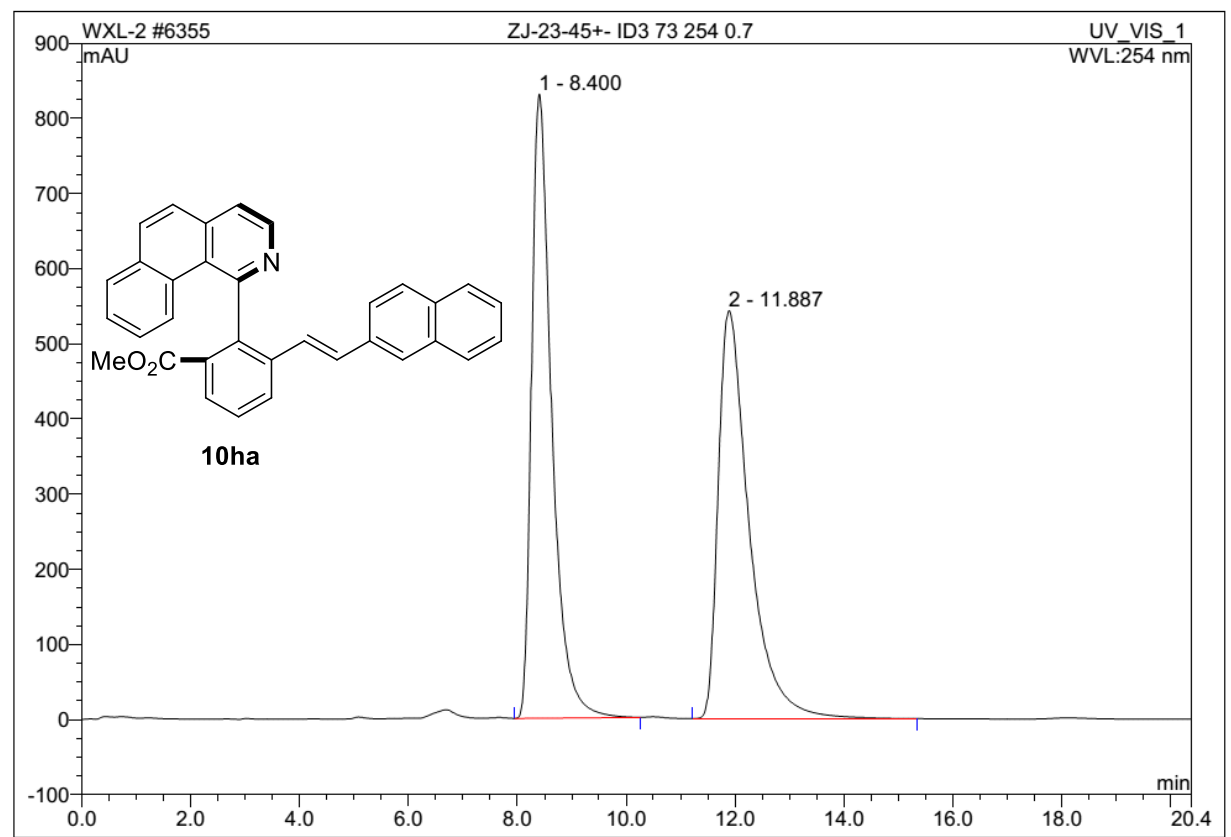

\begin{tabular}{|r|rrrrrrr|}
\hline No. & $\begin{array}{c}\text { Ret.Time } \\
\text { min }\end{array}$ & Peak Name & $\begin{array}{c}\text { Height } \\
\text { mAU }\end{array}$ & $\begin{array}{c}\text { Area } \\
\text { mAU*min }\end{array}$ & $\begin{array}{c}\text { Rel.Area } \\
\%\end{array}$ & Amount & Type \\
\hline 1 & 8.40 & n.a. & 830.890 & 356.346 & 49.99 & n.a. & BMB \\
2 & 11.89 & n.a. & 542.945 & 356.468 & 50.01 & n.a. & BMB \\
\hline Total: & & & 1373.835 & 712.813 & 100.00 & 0.000 & \\
\hline
\end{tabular}

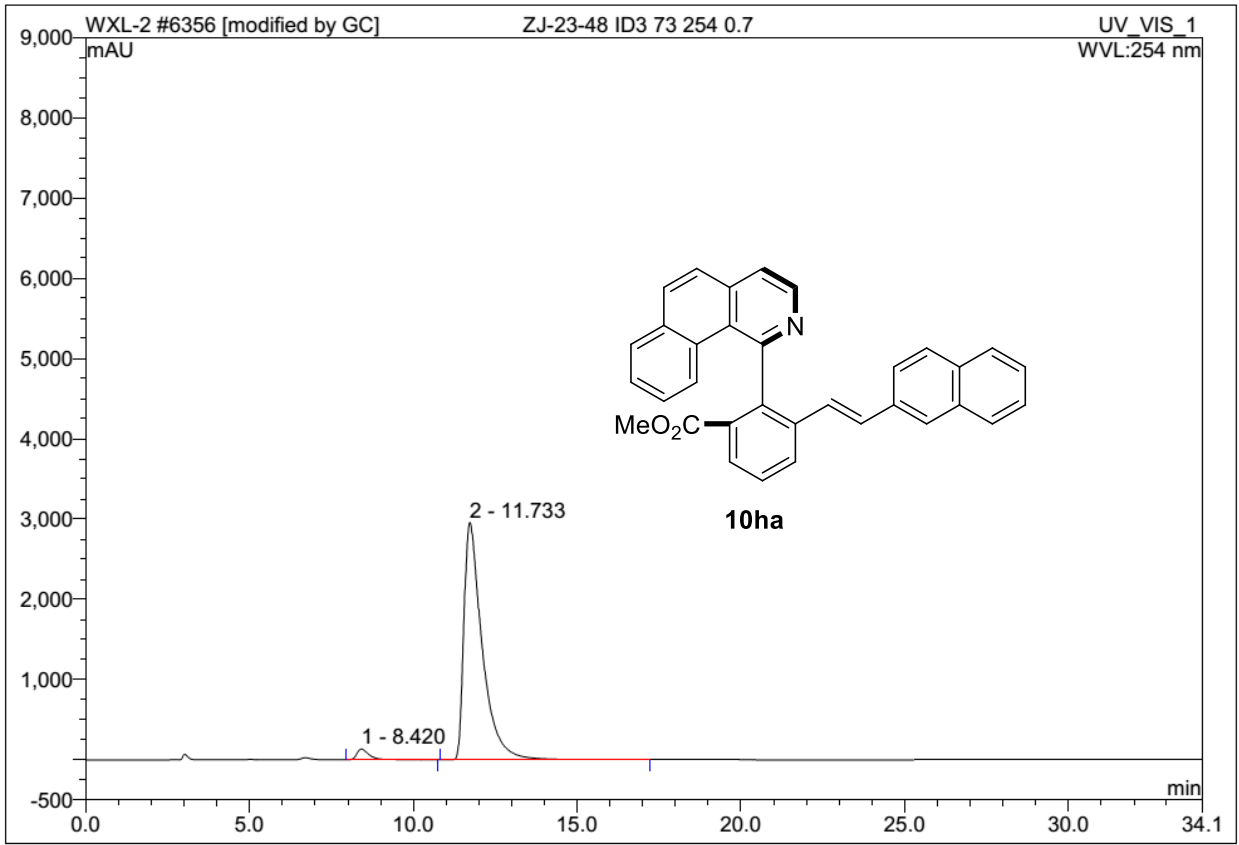

\begin{tabular}{|r|rrrrrrr|}
\hline No. & $\begin{array}{c}\text { Ret.Time } \\
\text { min }\end{array}$ & Peak Name & $\begin{array}{c}\text { Height } \\
\text { mAU }\end{array}$ & $\begin{array}{c}\text { Area } \\
\text { mAU*min }\end{array}$ & $\begin{array}{r}\text { Rel.Area } \\
\%\end{array}$ & Amount & Type \\
\hline 1 & 8.42 & n.a. & 136.286 & 60.879 & 3.06 & n.a. & BMB \\
2 & 11.73 & n.a. & 2960.928 & 1931.536 & 96.94 & n.a. & BMB \\
\hline Total: & & & 3097.214 & 1992.415 & 100.00 & 0.000 & \\
\hline
\end{tabular}




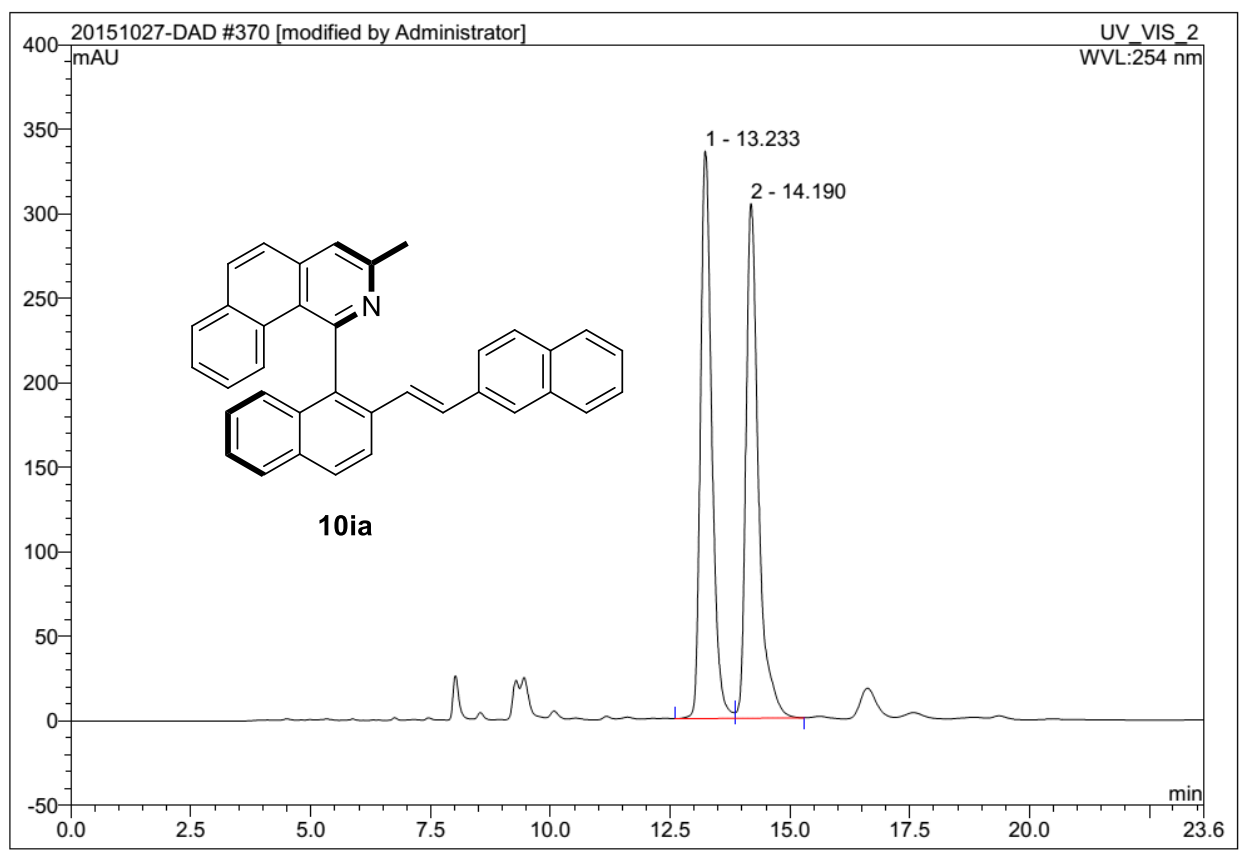

\begin{tabular}{|r|ccrrrrr|}
\hline No. & $\begin{array}{c}\text { Ret.Time } \\
\text { min }\end{array}$ & Peak Name & $\begin{array}{c}\text { Height } \\
\text { mAU }\end{array}$ & $\begin{array}{r}\text { Area } \\
\text { mAU*min }\end{array}$ & $\begin{array}{r}\text { Rel.Area } \\
\%\end{array}$ & Amount & Type \\
\hline 1 & 13.23 & n.a. & 335.949 & 92.148 & 50.04 & n.a. & BM $^{*}$ \\
2 & 14.19 & n.a. & 304.845 & 91.997 & 49.96 & n.a. & MB $^{*}$ \\
\hline Total: & & & 640.794 & 184.146 & 100.00 & 0.000 & \\
\hline
\end{tabular}

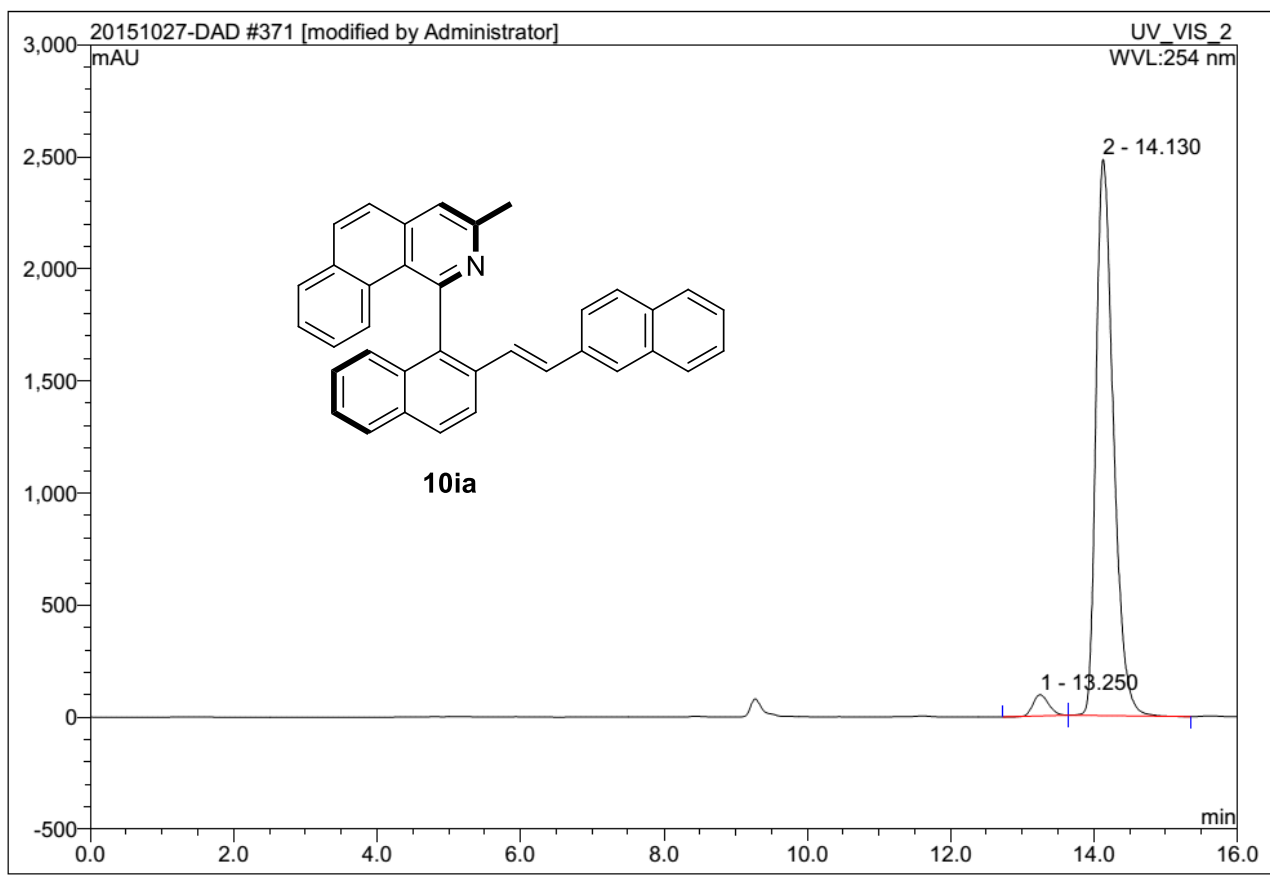

\begin{tabular}{|r|ccrrrrr|}
\hline No. & $\begin{array}{c}\text { Ret.Time } \\
\text { min }\end{array}$ & Peak Name & $\begin{array}{c}\text { Height } \\
\text { mAU }\end{array}$ & $\begin{array}{c}\text { Area } \\
\text { mAU*min }\end{array}$ & $\begin{array}{r}\text { Rel.Area } \\
\%\end{array}$ & Amount & Type \\
\hline 1 & 13.25 & n.a. & 95.062 & 24.085 & 3.33 & n.a. & BMB $^{*}$ \\
2 & 14.13 & n.a. & 2483.572 & 699.741 & 96.67 & n.a. & BMB $^{*}$ \\
\hline Total: & & & 2578.635 & 723.826 & 100.00 & 0.000 & \\
\hline
\end{tabular}




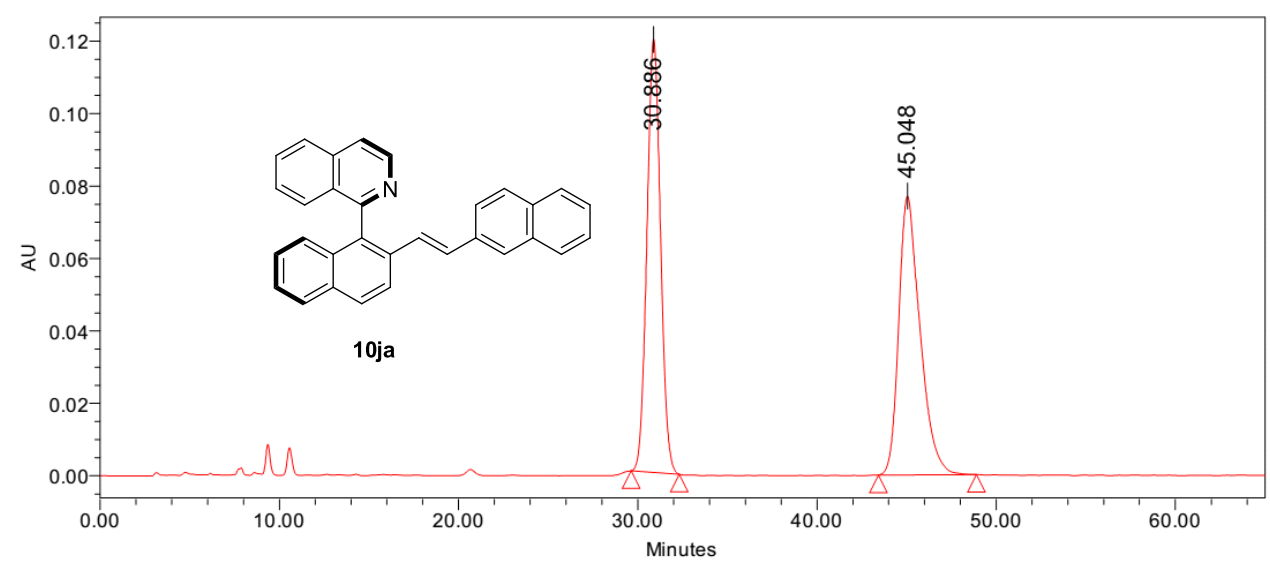

Peak Results
\begin{tabular}{|l|l|c|c|r|r|}
\hline & Name & RT & Area & Height & $\%$ Area \\
\hline 1 & & 30.886 & 6308309 & 119565 & 49.83 \\
\hline 2 & & 45.048 & 6351000 & 76977 & 50.17 \\
\hline
\end{tabular}

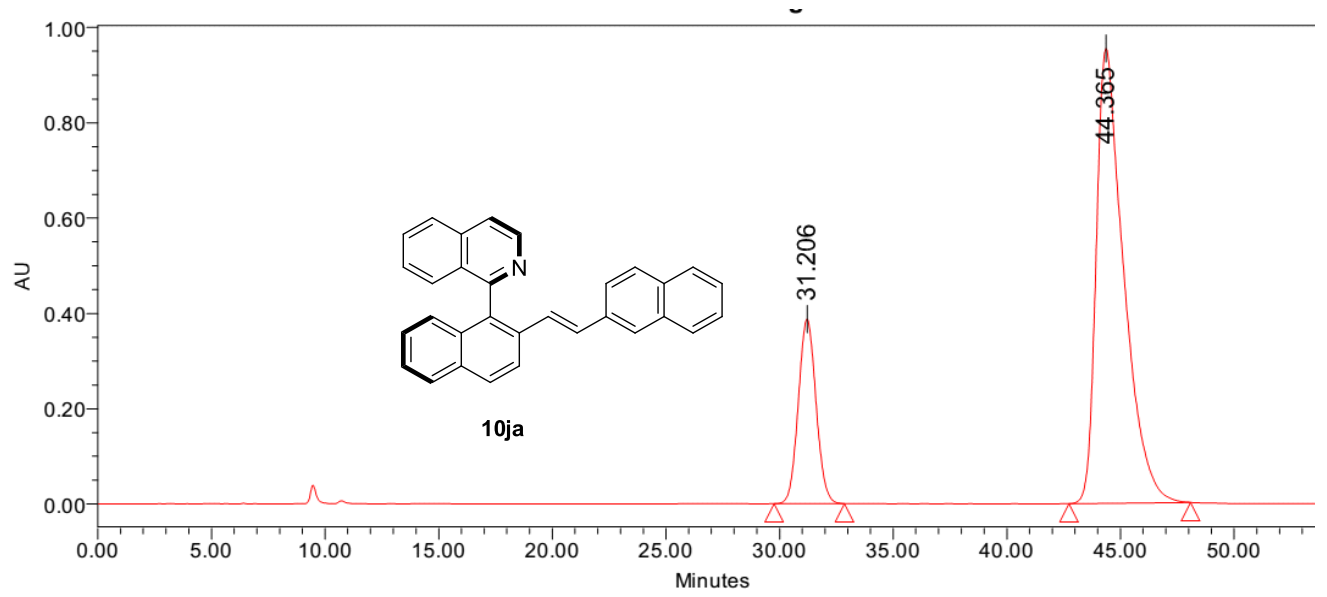

\section{Peak Results}

\begin{tabular}{|l|c|c|c|c|r|}
\hline & Name & RT & Area & Height & $\%$ Area \\
\hline 1 & & 31.206 & 21025313 & 387511 & 20.80 \\
\hline 2 & & 44.365 & 80066965 & 954427 & 79.20 \\
\hline
\end{tabular}




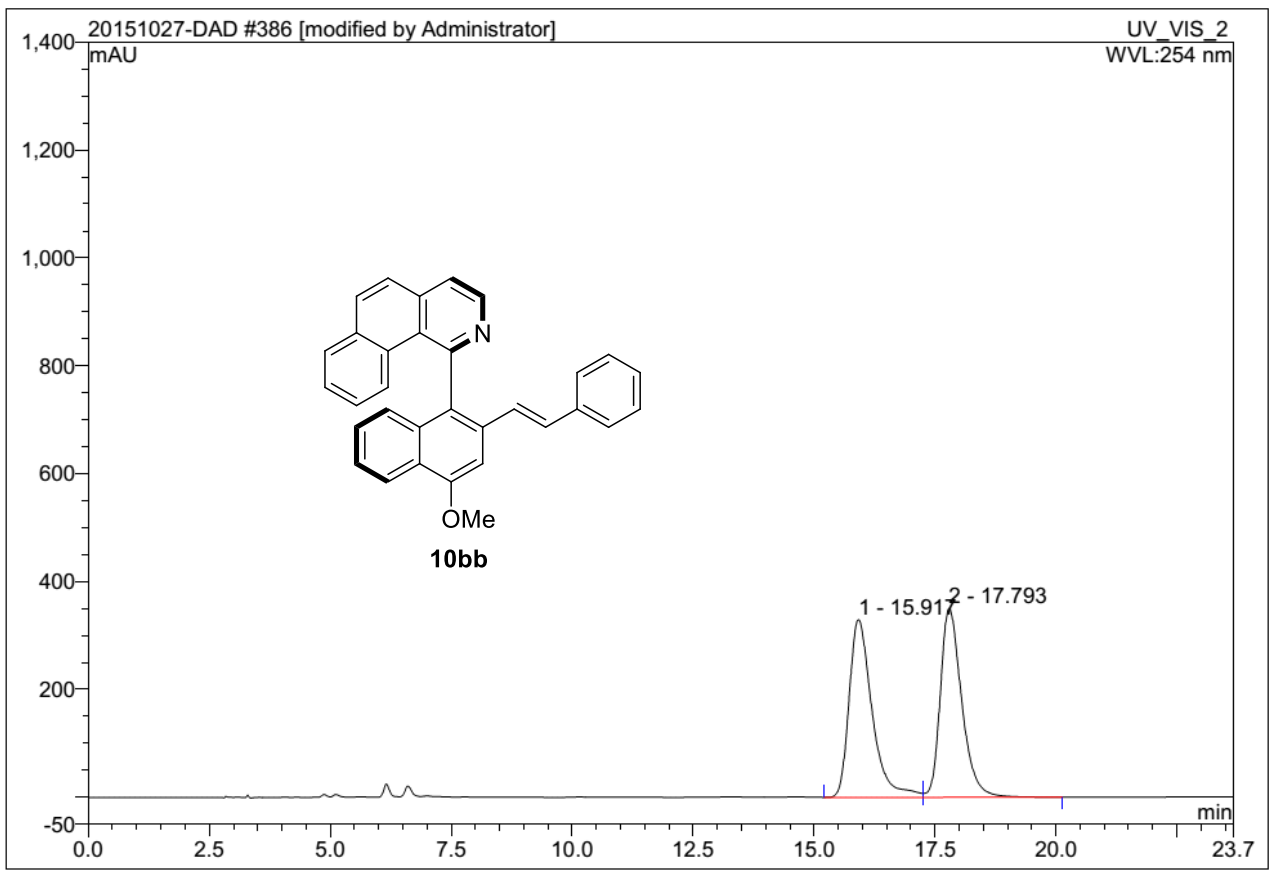

\begin{tabular}{|r|ccccccc|}
\hline No. & $\begin{array}{c}\text { Ret.Time } \\
\text { min }\end{array}$ & Peak Name & $\begin{array}{c}\text { Height } \\
\text { mAU }\end{array}$ & $\begin{array}{c}\text { Area } \\
\text { mAU*min }\end{array}$ & $\begin{array}{c}\text { Rel.Area } \\
\%\end{array}$ & Amount & Type \\
\hline 1 & 15.92 & n.a. & 329.645 & 181.972 & 50.24 & n.a. & BM \\
2 & 17.79 & n.a. & 350.722 & 180.254 & 49.76 & n.a. & MB \\
\hline Total: & & & 680.367 & 362.226 & 100.00 & 0.000 & \\
\hline
\end{tabular}

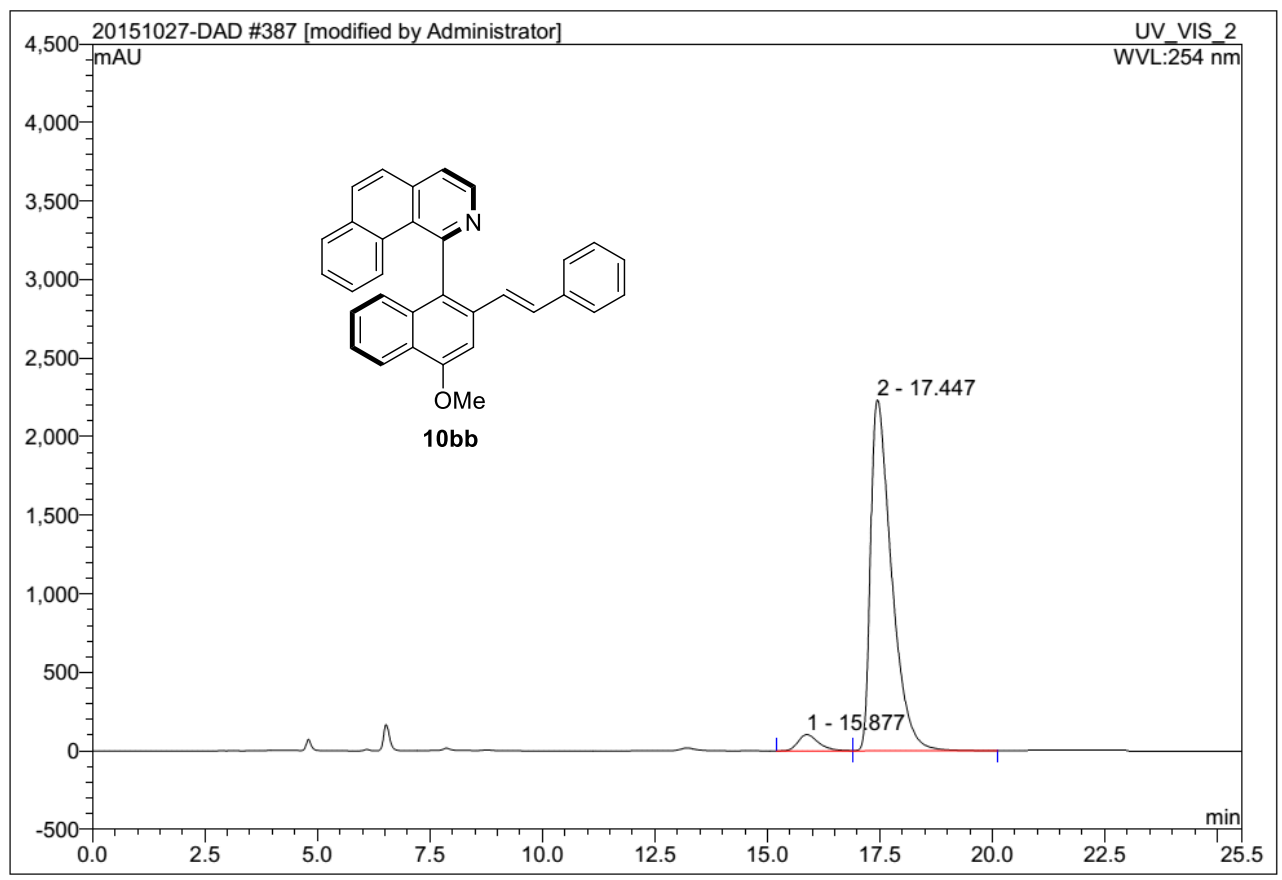

\begin{tabular}{|r|ccrrrrr|}
\hline No. & $\begin{array}{c}\text { Ret.Time } \\
\text { min }\end{array}$ & Peak Name & $\begin{array}{c}\text { Height } \\
\text { mAU }\end{array}$ & $\begin{array}{c}\text { Area } \\
\text { mAU*min }\end{array}$ & $\begin{array}{r}\text { Rel.Area } \\
\%\end{array}$ & Amount & Type \\
\hline 1 & 15.88 & n.a. & 103.450 & 55.180 & 4.36 & n.a. & BM \\
2 & 17.45 & n.a. & 2234.552 & 1209.687 & 95.64 & n.a. & MB \\
\hline Total: & & & 2338.002 & 1264.866 & 100.00 & 0.000 & \\
\hline
\end{tabular}




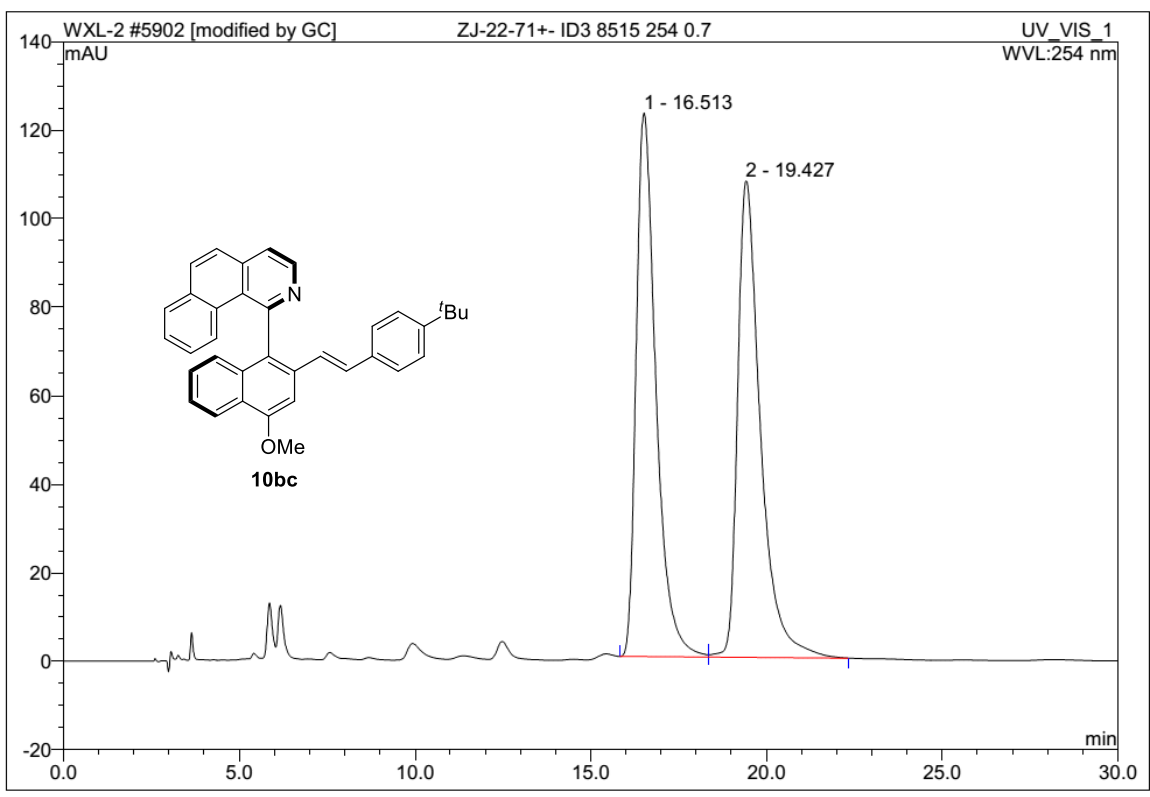

\begin{tabular}{|r|cccrrrr|}
\hline No. & $\begin{array}{c}\text { Ret.Time } \\
\text { min }\end{array}$ & Peak Name & $\begin{array}{c}\text { Height } \\
\text { mAU }\end{array}$ & $\begin{array}{r}\text { Area } \\
\text { mAU*min }\end{array}$ & $\begin{array}{r}\text { Rel.Area } \\
\%\end{array}$ & Amount & Type \\
\hline 1 & 16.51 & n.a. & 122.926 & 80.157 & 49.66 & n.a. & BM \\
2 & 19.43 & n.a. & 107.751 & 81.253 & 50.34 & n.a. & MB \\
\hline Total: & & & 230.677 & 161.410 & 100.00 & 0.000 & \\
\hline
\end{tabular}

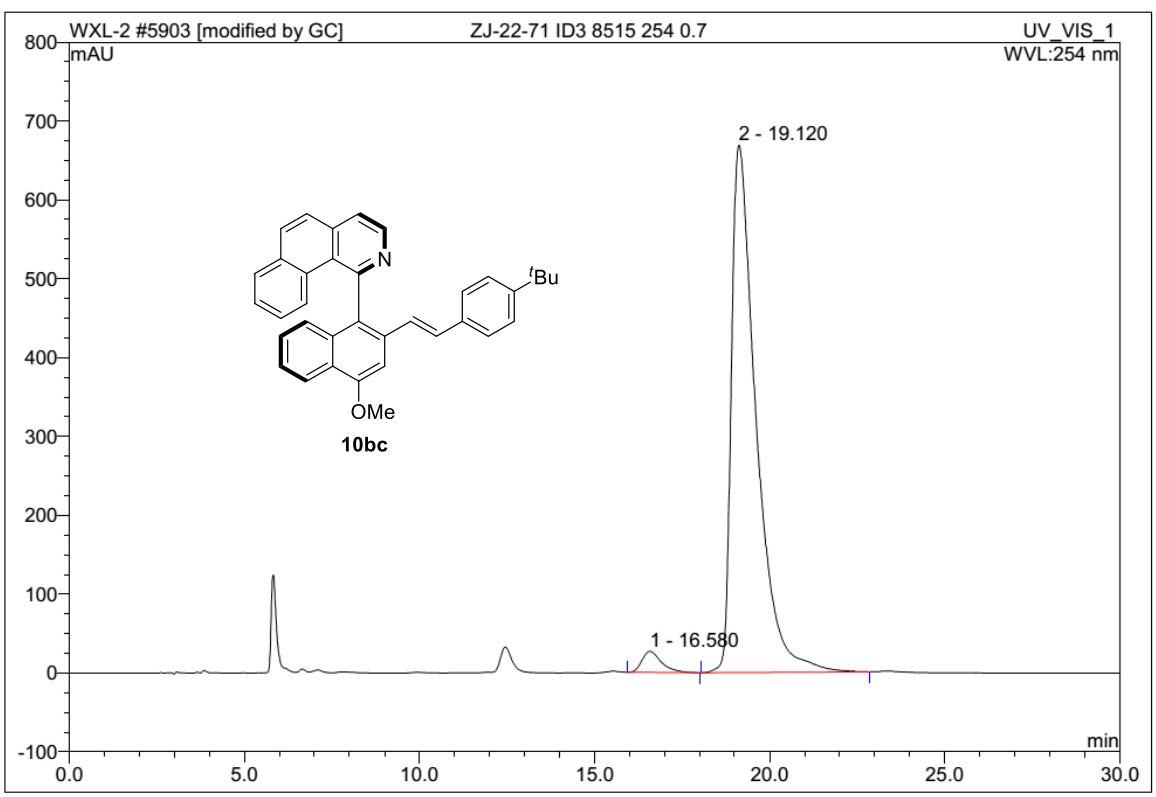

\begin{tabular}{r|ccrrrrr|}
\hline No. & $\begin{array}{c}\text { Ret.Time } \\
\text { min }\end{array}$ & Peak Name & $\begin{array}{c}\text { Height } \\
\text { mAU }\end{array}$ & $\begin{array}{c}\text { Area } \\
\text { mAU*min }\end{array}$ & $\begin{array}{r}\text { Rel.Area } \\
\%\end{array}$ & Amount & Type \\
\hline 1 & 16.58 & n.a. & 26.579 & 17.050 & 3.03 & n.a. & BMB \\
2 & 19.12 & n.a. & 669.434 & 545.320 & 96.97 & n.a. & BMB \\
\hline Total: & & & 696.013 & 562.370 & 100.00 & 0.000 & \\
\hline
\end{tabular}




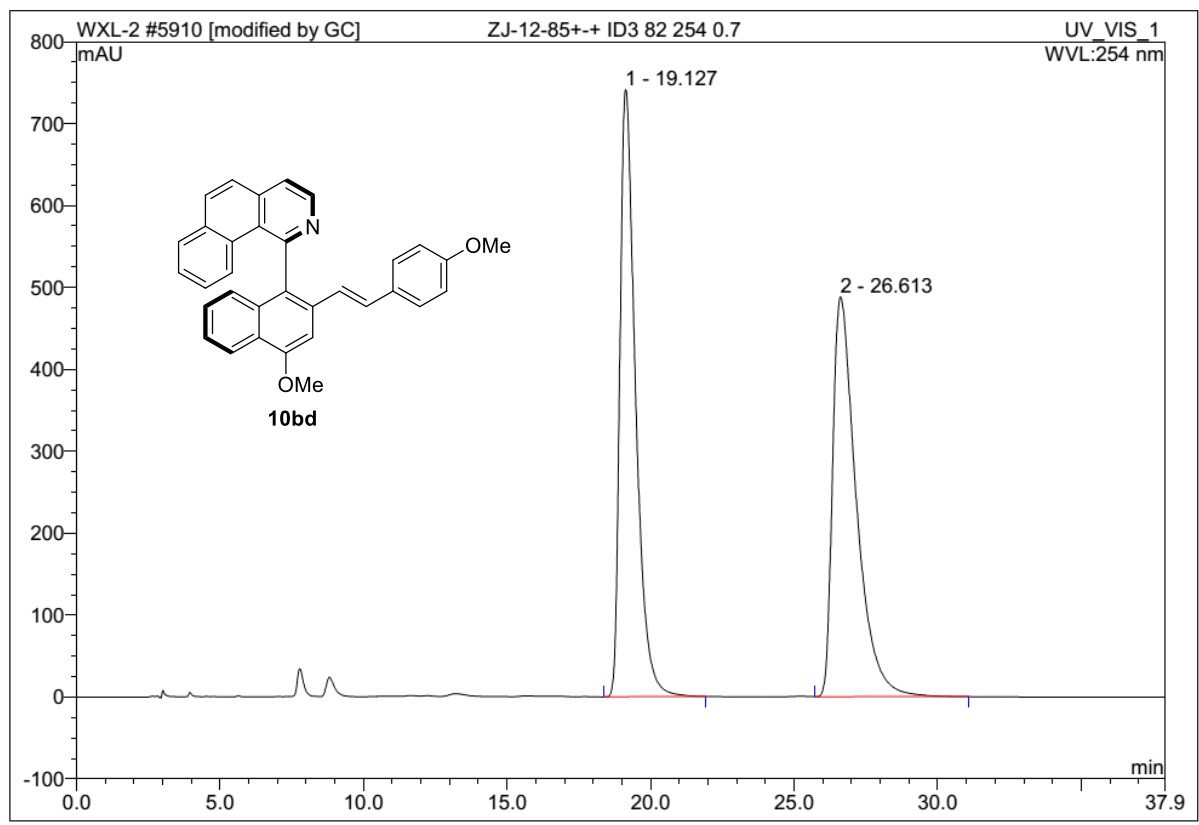

\begin{tabular}{|r|ccccccc|}
\hline No. & $\begin{array}{c}\text { Ret.Time } \\
\text { min }\end{array}$ & Peak Name & $\begin{array}{c}\text { Height } \\
\text { mAU }\end{array}$ & $\begin{array}{c}\text { Area } \\
\text { mAU*min }\end{array}$ & $\begin{array}{c}\text { Rel.Area } \\
\%\end{array}$ & Amount & Type \\
\hline 1 & 19.13 & n.a. & 741.663 & 468.575 & 50.12 & n.a. & BMB \\
2 & 26.61 & n.a. & 488.254 & 466.360 & 49.88 & n.a. & BMB \\
\hline Total: & & & 1229.918 & 934.935 & 100.00 & 0.000 & \\
\hline
\end{tabular}

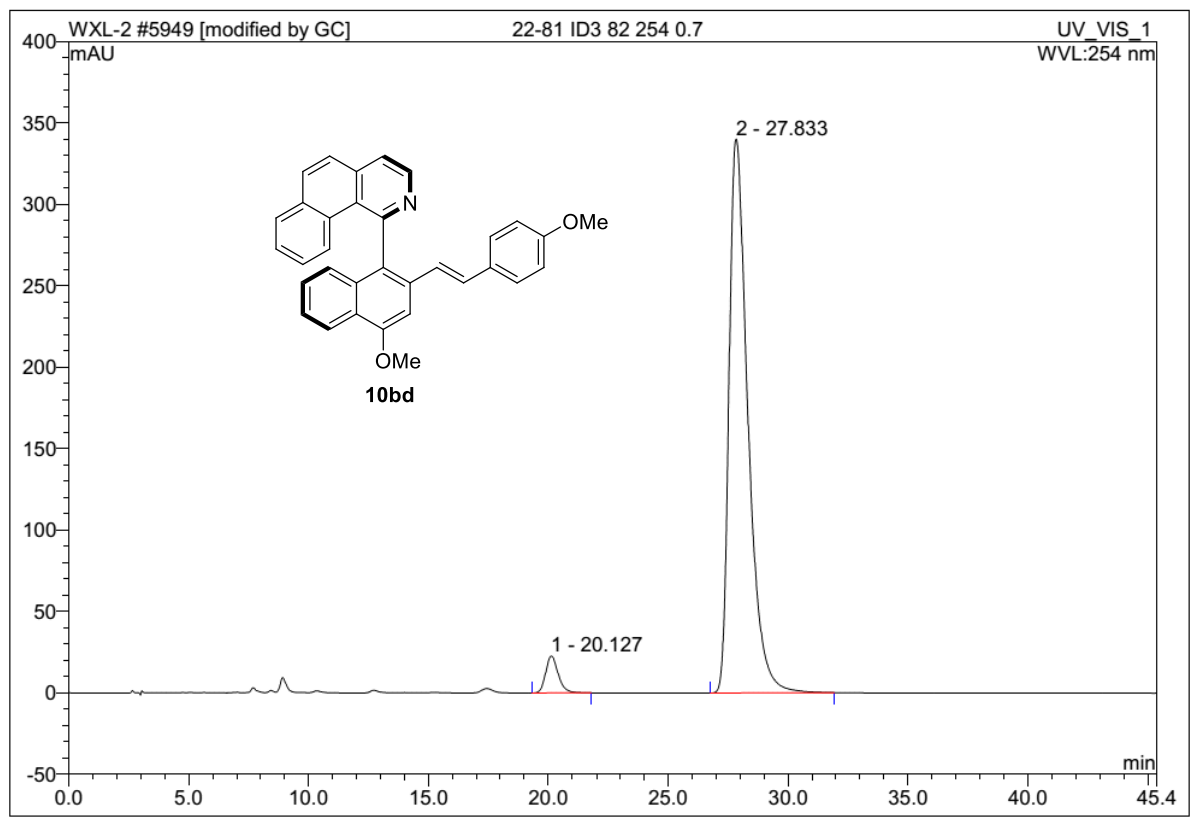

\begin{tabular}{|r|ccrrrrr|}
\hline No. & $\begin{array}{c}\text { Ret.Time } \\
\text { min }\end{array}$ & Peak Name & $\begin{array}{c}\text { Height } \\
\text { mAU }\end{array}$ & $\begin{array}{r}\text { Area } \\
\text { mAU*min }\end{array}$ & $\begin{array}{r}\text { Rel.Area } \\
\%\end{array}$ & Amount & Type \\
\hline 1 & 20.13 & n.a. & 22.563 & 13.727 & 4.25 & n.a. & BMB \\
2 & 27.83 & n.a. & 340.222 & 309.544 & 95.75 & n.a. & BMB \\
\hline Total: & & & 362.785 & 323.272 & 100.00 & 0.000 & \\
\hline
\end{tabular}




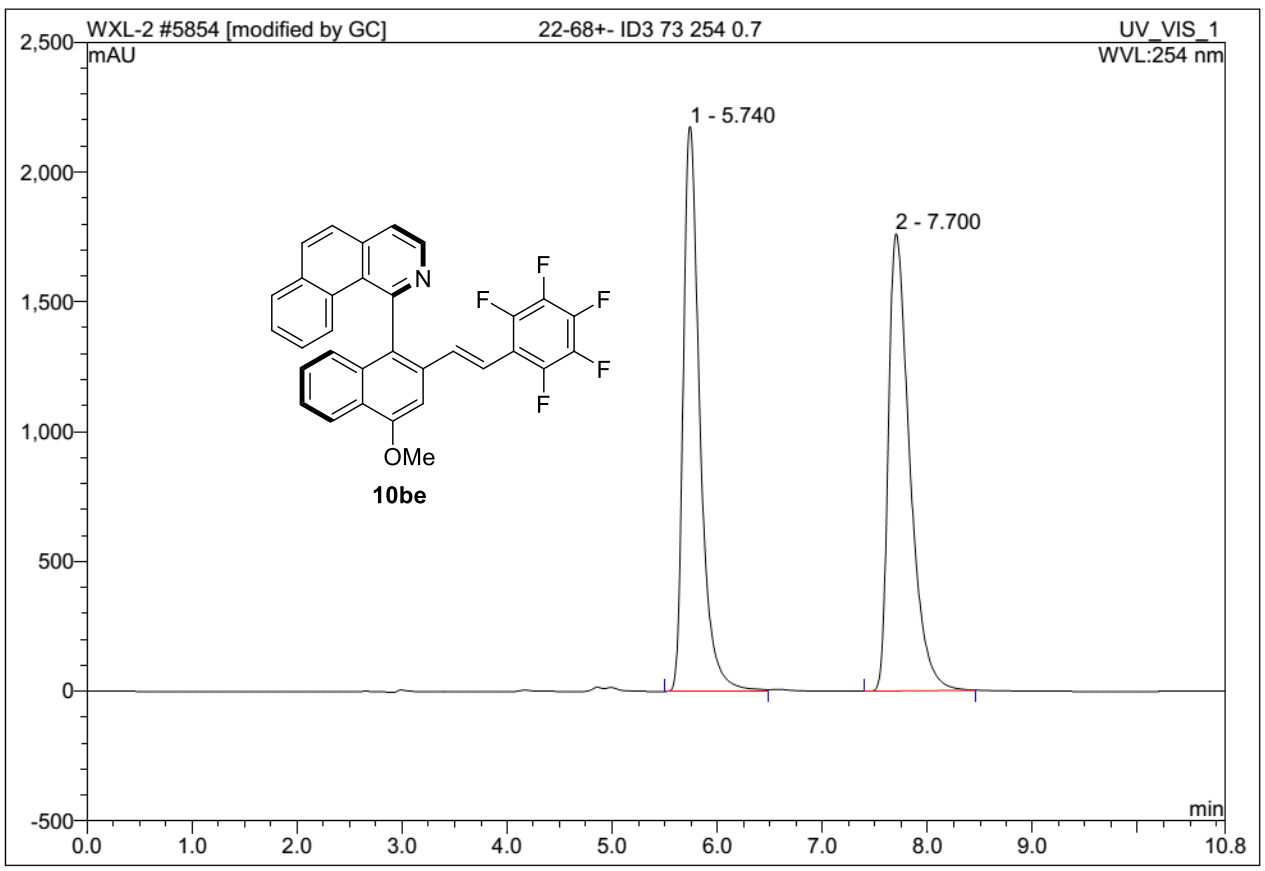

\begin{tabular}{|r|cccrrrr|}
\hline No. & $\begin{array}{c}\text { Ret.Time } \\
\text { min }\end{array}$ & Peak Name & $\begin{array}{c}\text { Height } \\
\text { mAU }\end{array}$ & $\begin{array}{c}\text { Area } \\
\text { mAU*min }\end{array}$ & $\begin{array}{c}\text { Rel.Area } \\
\%\end{array}$ & Amount & Type \\
\hline 1 & 5.74 & n.a. & 2176.531 & 401.546 & 49.16 & n.a. & BM $^{*}$ \\
2 & 7.70 & n.a. & 1763.917 & 415.282 & 50.84 & n.a. & BMB $^{\star}$ \\
\hline Total: & & & 3940.448 & 816.827 & 100.00 & 0.000 & \\
\hline
\end{tabular}

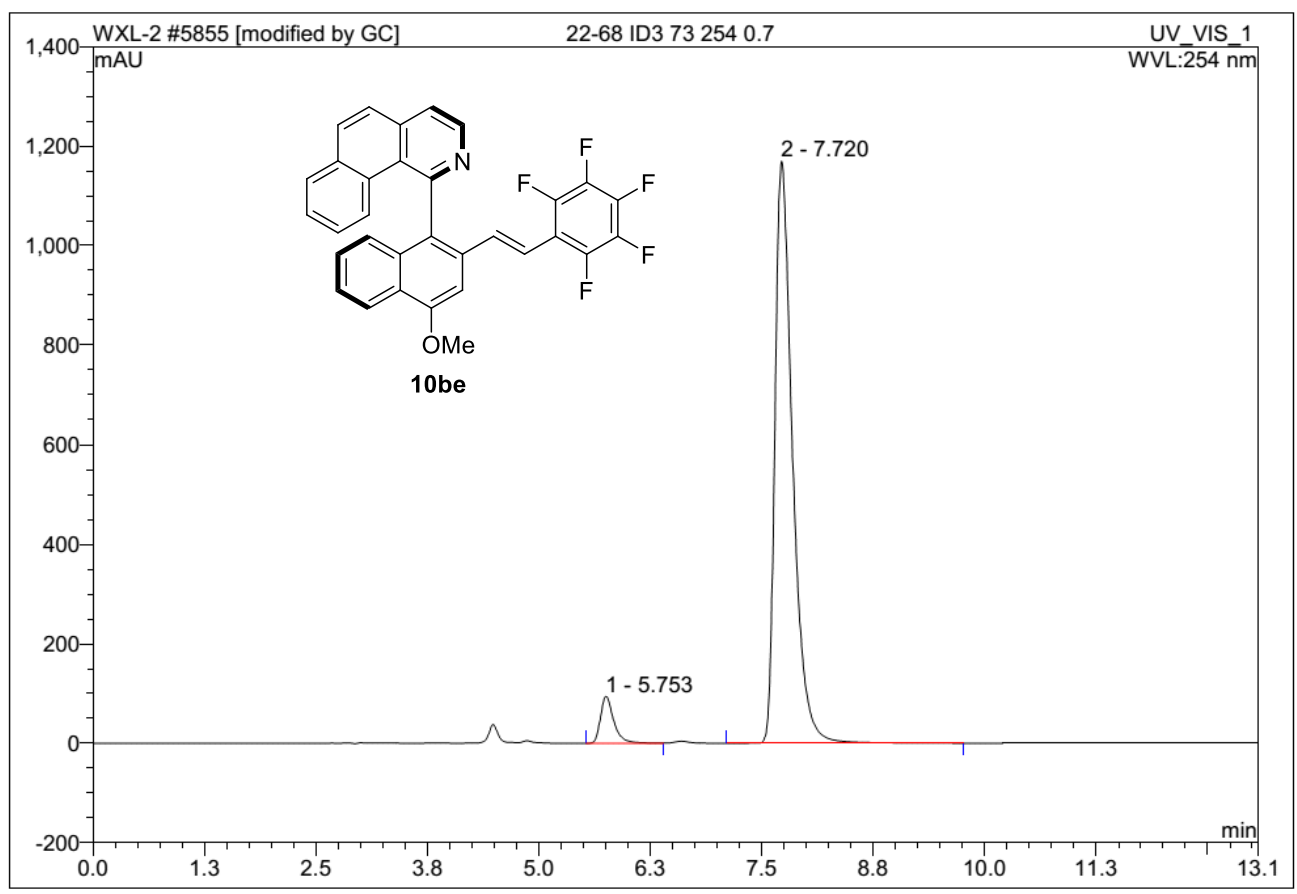

\begin{tabular}{|r|ccrrrrr|}
\hline No. & $\begin{array}{c}\text { Ret.Time } \\
\text { min }\end{array}$ & Peak Name & $\begin{array}{c}\text { Height } \\
\text { mAU }\end{array}$ & $\begin{array}{c}\text { Area } \\
\text { mAU*min }\end{array}$ & $\begin{array}{r}\text { Rel.Area } \\
\%\end{array}$ & Amount & Type \\
\hline 1 & 5.75 & n.a. & 94.376 & 16.296 & 5.74 & n.a. & BMB \\
2 & 7.72 & n.a. & 1170.843 & 267.751 & 94.26 & n.a. & BMB \\
\hline Total: & & & 1265.219 & 284.047 & 100.00 & 0.000 & \\
\hline
\end{tabular}




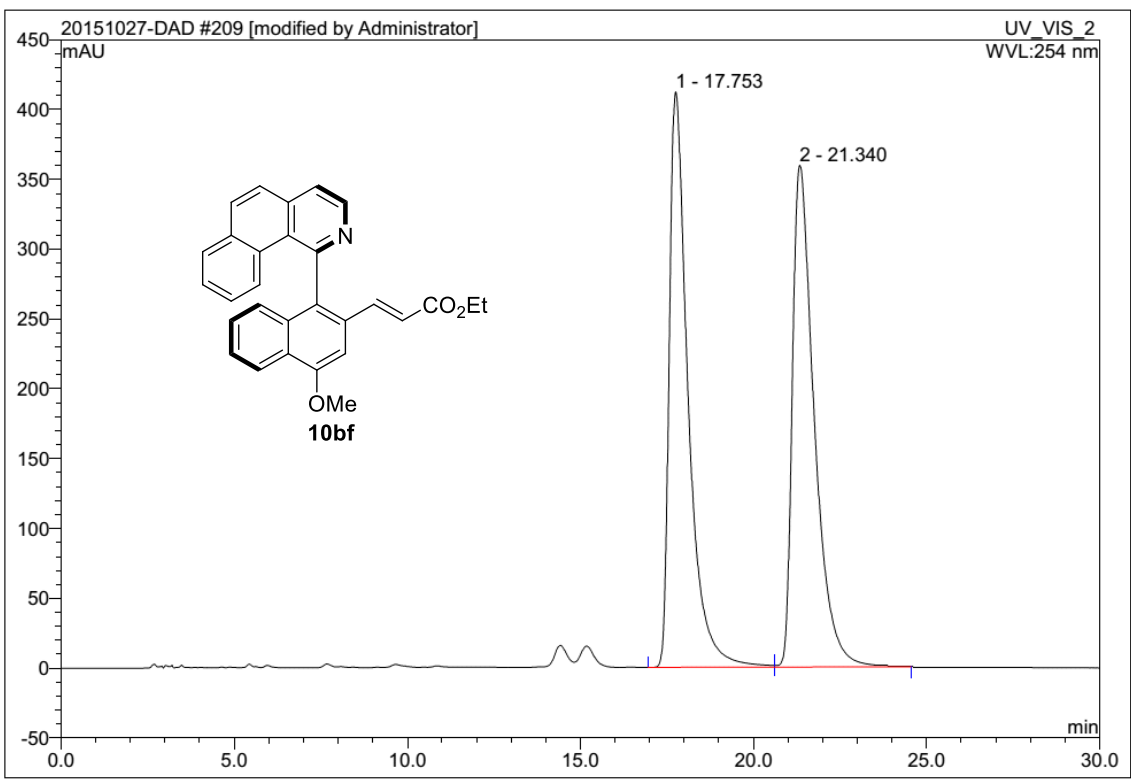

\begin{tabular}{|r|ccccccc|}
\hline No. & $\begin{array}{c}\text { Ret.Time } \\
\text { min }\end{array}$ & Peak Name & $\begin{array}{c}\text { Height } \\
\text { mAU }\end{array}$ & $\begin{array}{c}\text { Area } \\
\text { mAU*min }\end{array}$ & $\begin{array}{c}\text { Rel.Area } \\
\%\end{array}$ & Amount & Type \\
\hline 1 & 17.75 & n.a. & 412.297 & 260.296 & 49.75 & n.a. & BM \\
2 & 21.34 & n.a. & 359.498 & 262.886 & 50.25 & n.a. & MB \\
\hline Total: & & & 771.795 & 523.182 & 100.00 & 0.000 & \\
\hline
\end{tabular}

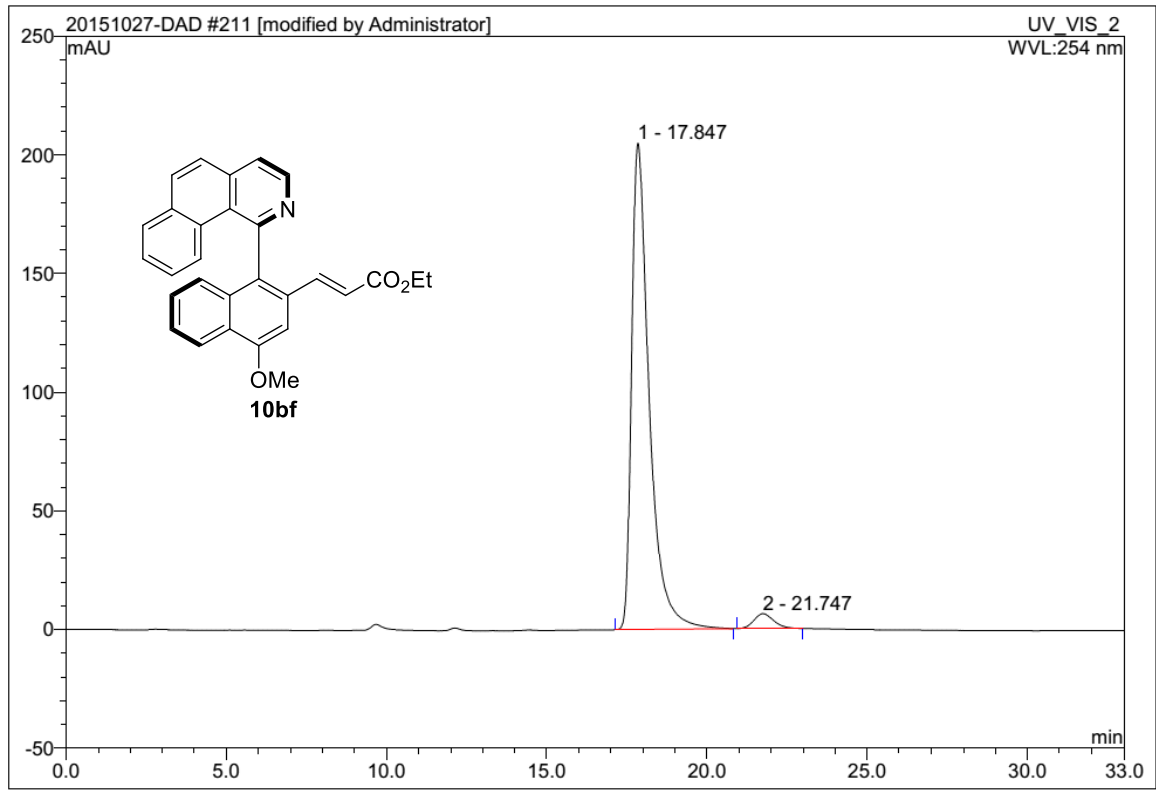

\begin{tabular}{r|ccrrrrr}
\hline No. & $\begin{array}{c}\text { Ret.Time } \\
\text { min }\end{array}$ & Peak Name & $\begin{array}{c}\text { Height } \\
\text { mAU }\end{array}$ & $\begin{array}{c}\text { Area } \\
\text { mAU*min }\end{array}$ & $\begin{array}{r}\text { Rel.Area } \\
\%\end{array}$ & Amount & Type \\
\hline 1 & 17.85 & n.a. & 204.992 & 129.981 & 96.60 & n.a. & BMB \\
2 & 21.75 & n.a. & 6.263 & 4.576 & 3.40 & n.a. & BMB \\
\hline Total: & & & 211.255 & 134.557 & 100.00 & 0.000 & \\
\hline
\end{tabular}




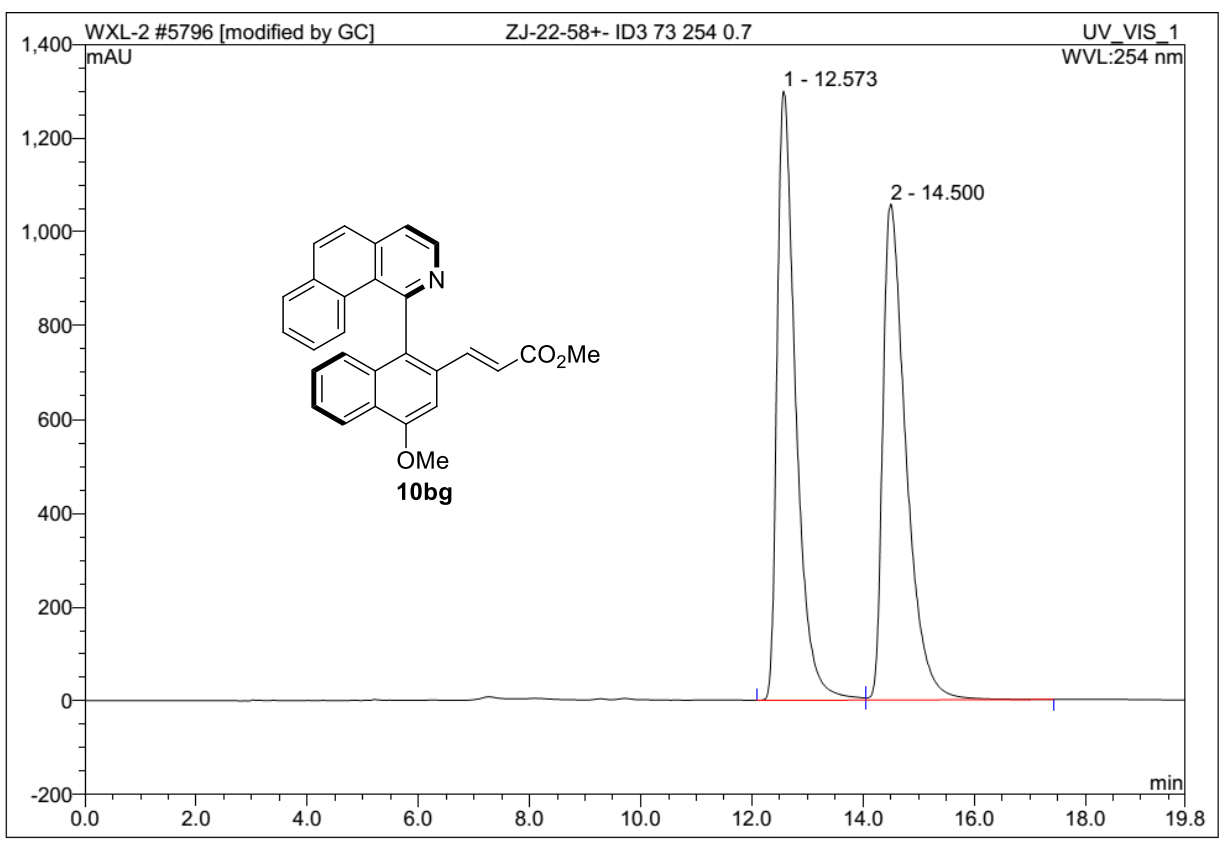

\begin{tabular}{|r|ccrrrrr|}
\hline No. & $\begin{array}{c}\text { Ret.Time } \\
\text { min }\end{array}$ & Peak Name & $\begin{array}{c}\text { Height } \\
\text { mAU }\end{array}$ & $\begin{array}{c}\text { Area } \\
\text { mAU*min }\end{array}$ & $\begin{array}{c}\text { Rel.Area } \\
\%\end{array}$ & Amount & Type \\
\hline 1 & 12.57 & n.a. & 1299.669 & 507.614 & 49.78 & n.a. & BM \\
2 & 14.50 & n.a. & 1058.013 & 512.110 & 50.22 & n.a. & MB \\
\hline Total: & & & 2357.682 & 1019.725 & 100.00 & 0.000 & \\
\hline
\end{tabular}

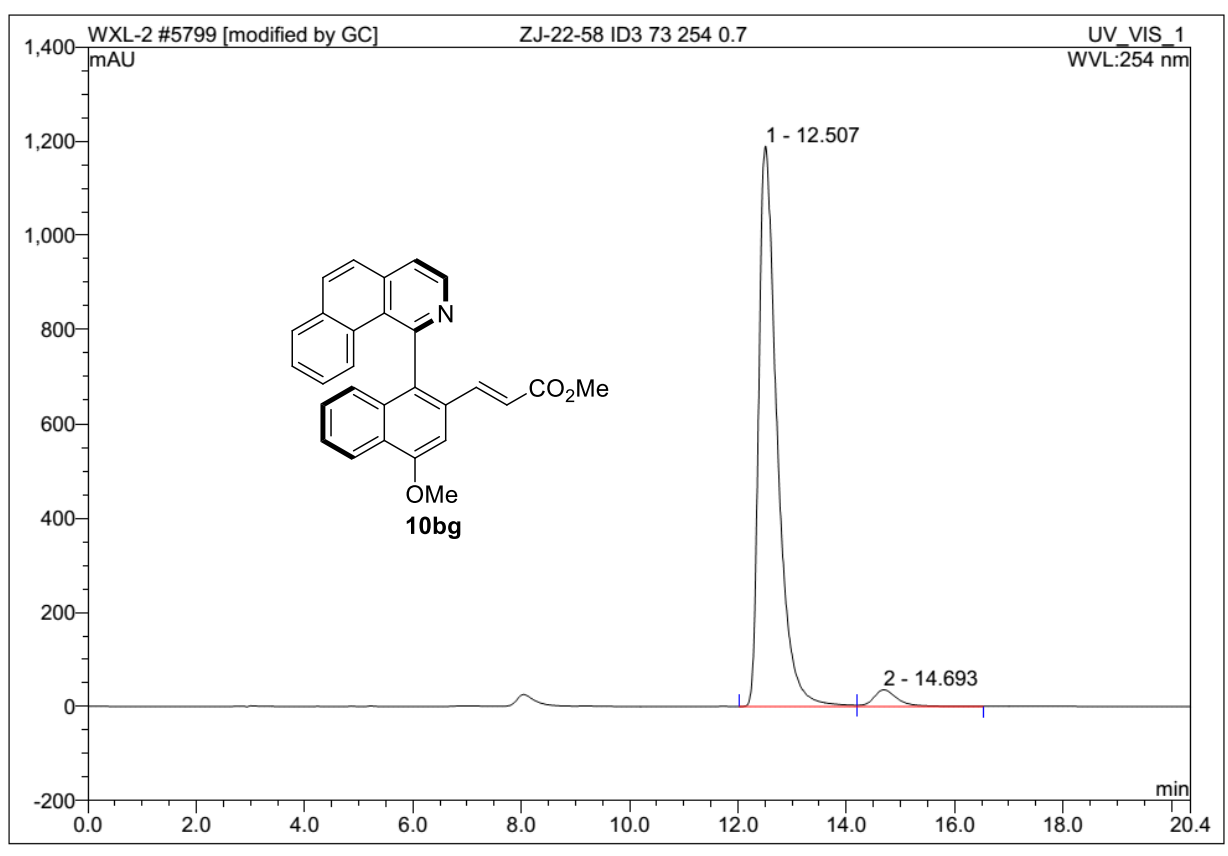

\begin{tabular}{r|rrrrrrr|}
\hline No. & $\begin{array}{c}\text { Ret.Time } \\
\text { min }\end{array}$ & Peak Name & $\begin{array}{c}\text { Height } \\
\text { mAU }\end{array}$ & $\begin{array}{r}\text { Area } \\
\text { mAU*min }\end{array}$ & $\begin{array}{r}\text { Rel.Area } \\
\%\end{array}$ & Amount & Type \\
\hline 1 & 12.51 & n.a. & 1190.420 & 467.214 & 96.23 & n.a. & BM \\
2 & 14.69 & n.a. & 35.477 & 18.298 & 3.77 & n.a. & MB \\
\hline Total: & & & 1225.897 & 485.512 & 100.00 & 0.000 & \\
\hline
\end{tabular}




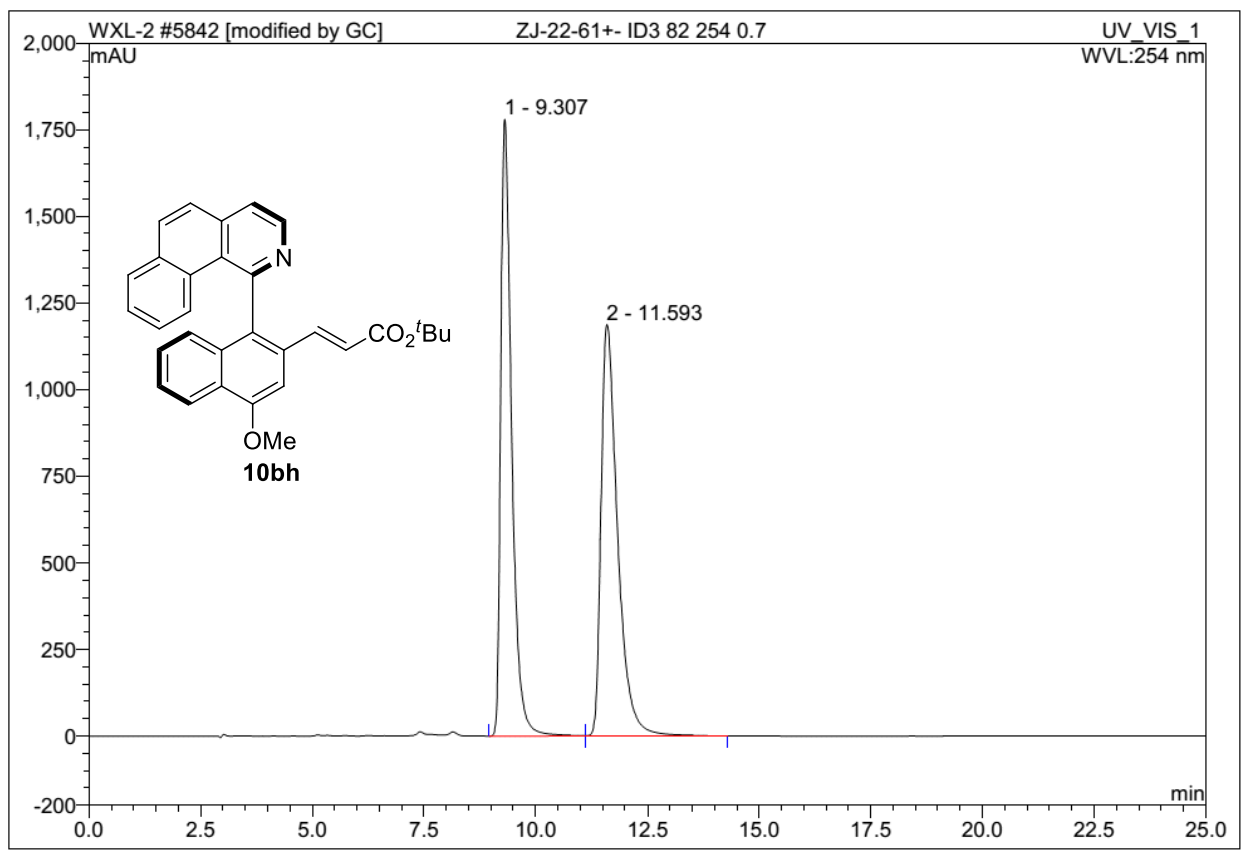

\begin{tabular}{r|ccrrrrr|}
\hline No. & $\begin{array}{c}\text { Ret.Time } \\
\text { min }\end{array}$ & Peak Name & $\begin{array}{c}\text { Height } \\
\text { mAU }\end{array}$ & $\begin{array}{c}\text { Area } \\
\text { mAU*min }\end{array}$ & $\begin{array}{c}\text { Rel.Area } \\
\%\end{array}$ & Amount & Type \\
\hline 1 & 9.31 & n.a. & 1781.099 & 504.036 & 49.77 & n.a. & BM \\
2 & 11.59 & n.a. & 1187.878 & 508.619 & 50.23 & n.a. & MB \\
\hline Total: & & & 2968.978 & 1012.654 & 100.00 & 0.000 & \\
\hline
\end{tabular}

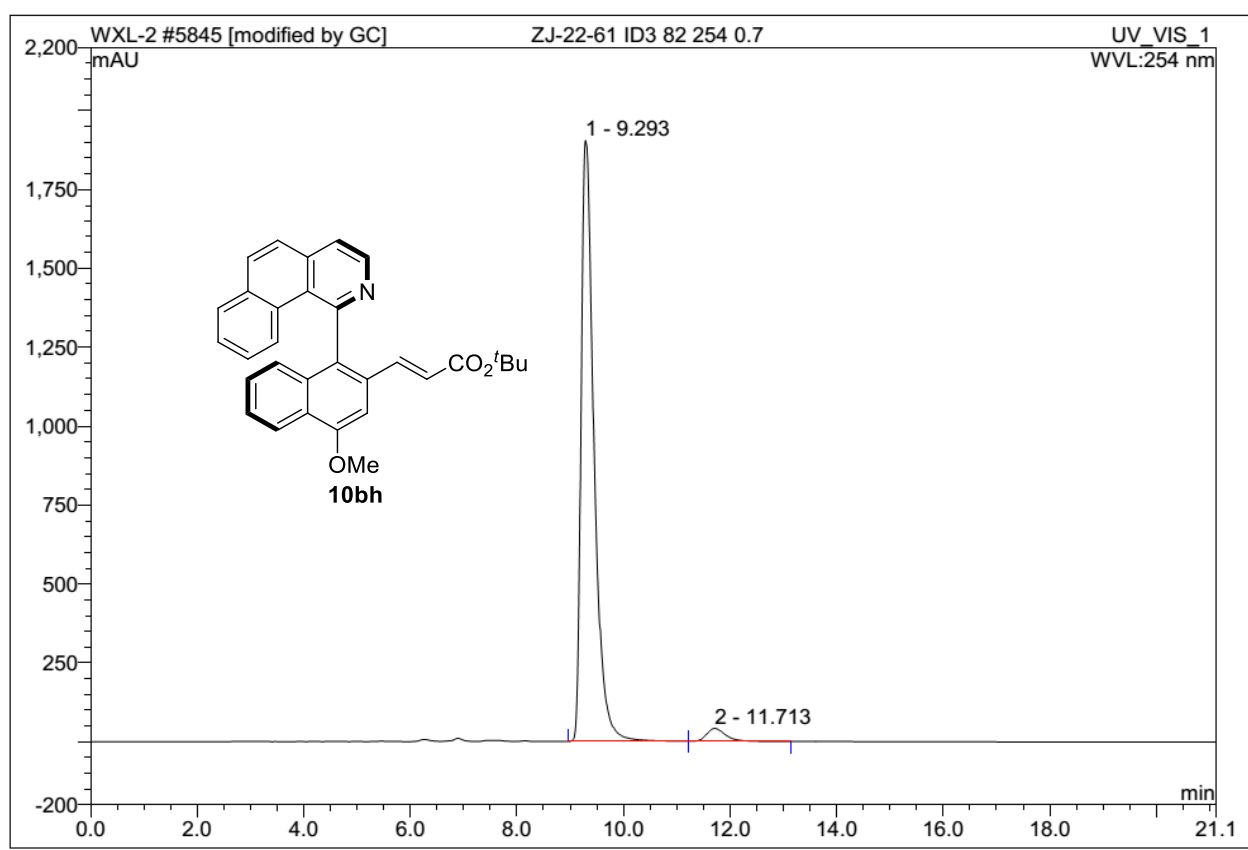

\begin{tabular}{|r|rrrrrrr|}
\hline No. & $\begin{array}{c}\text { Ret.Time } \\
\text { min }\end{array}$ & Peak Name & $\begin{array}{c}\text { Height } \\
\text { mAU }\end{array}$ & $\begin{array}{r}\text { Area } \\
\text { mAU*min }\end{array}$ & $\begin{array}{c}\text { Rel.Area } \\
\%\end{array}$ & Amount & Type \\
\hline 1 & 9.29 & n.a. & 1905.592 & 533.876 & 96.76 & n.a. & BM \\
2 & 11.71 & n.a. & 41.975 & 17.902 & 3.24 & n.a. & MB \\
\hline Total: & & & 1947.567 & 551.778 & 100.00 & 0.000 & \\
\hline
\end{tabular}




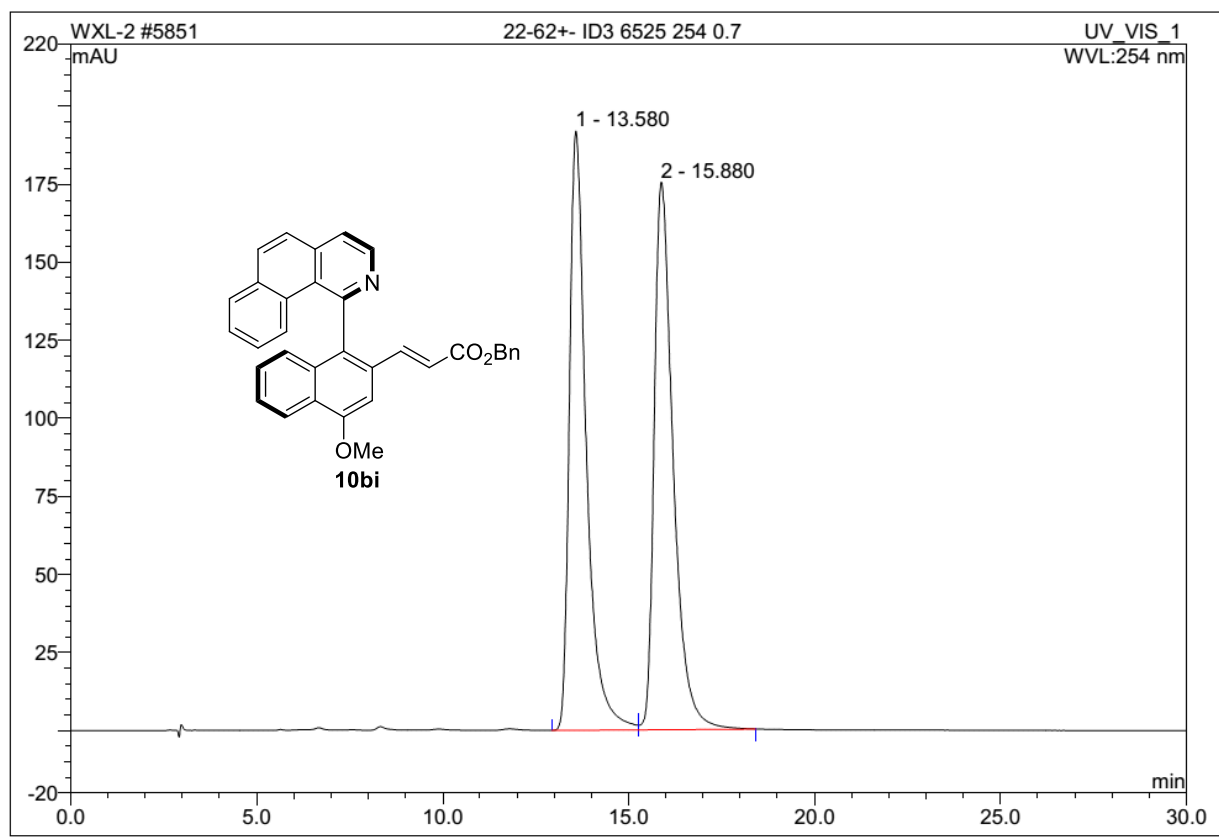

\begin{tabular}{|r|ccccccc|}
\hline No. & $\begin{array}{c}\text { Ret.Time } \\
\text { min }\end{array}$ & Peak Name & $\begin{array}{c}\text { Height } \\
\text { mAU }\end{array}$ & $\begin{array}{c}\text { Area } \\
\text { mAU*min }\end{array}$ & $\begin{array}{c}\text { Rel.Area } \\
\%\end{array}$ & Amount & Type \\
\hline 1 & 13.58 & n.a. & 192.000 & 101.139 & 49.41 & n.a. & BM \\
2 & 15.88 & n.a. & 175.555 & 103.568 & 50.59 & n.a. & MB \\
\hline Total: & & & 367.555 & 204.706 & 100.00 & 0.000 & \\
\hline
\end{tabular}

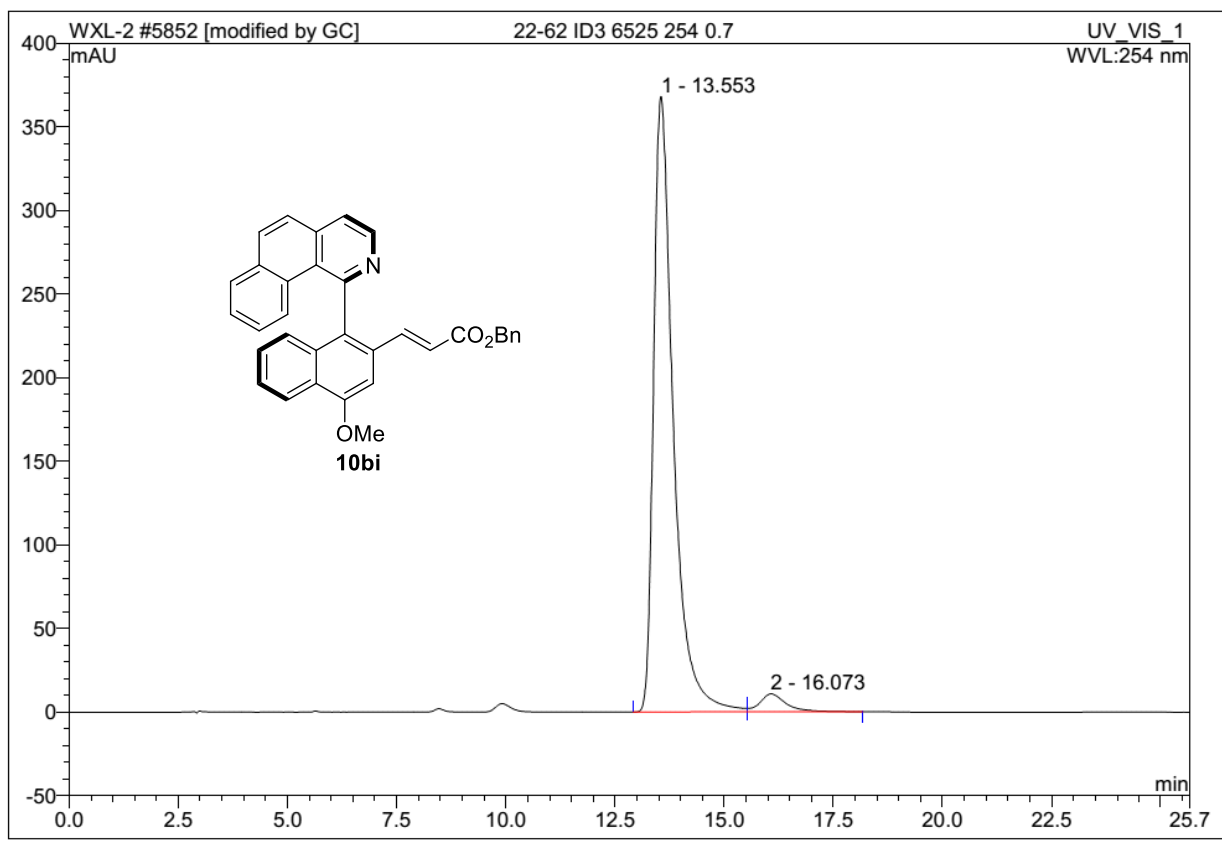

\begin{tabular}{|r|rrrrrrr|}
\hline No. & $\begin{array}{c}\text { Ret.Time } \\
\text { min }\end{array}$ & Peak Name & $\begin{array}{c}\text { Height } \\
\text { mAU }\end{array}$ & $\begin{array}{c}\text { Area } \\
\text { mAU*min }\end{array}$ & $\begin{array}{c}\text { Rel.Area } \\
\%\end{array}$ & Amount & Type \\
\hline 1 & 13.55 & n.a. & 368.215 & 192.583 & 96.26 & n.a. & BM \\
2 & 16.07 & n.a. & 10.674 & 7.483 & 3.74 & n.a. & MB \\
\hline Total: & & & 378.889 & 200.066 & 100.00 & 0.000 & \\
\hline
\end{tabular}




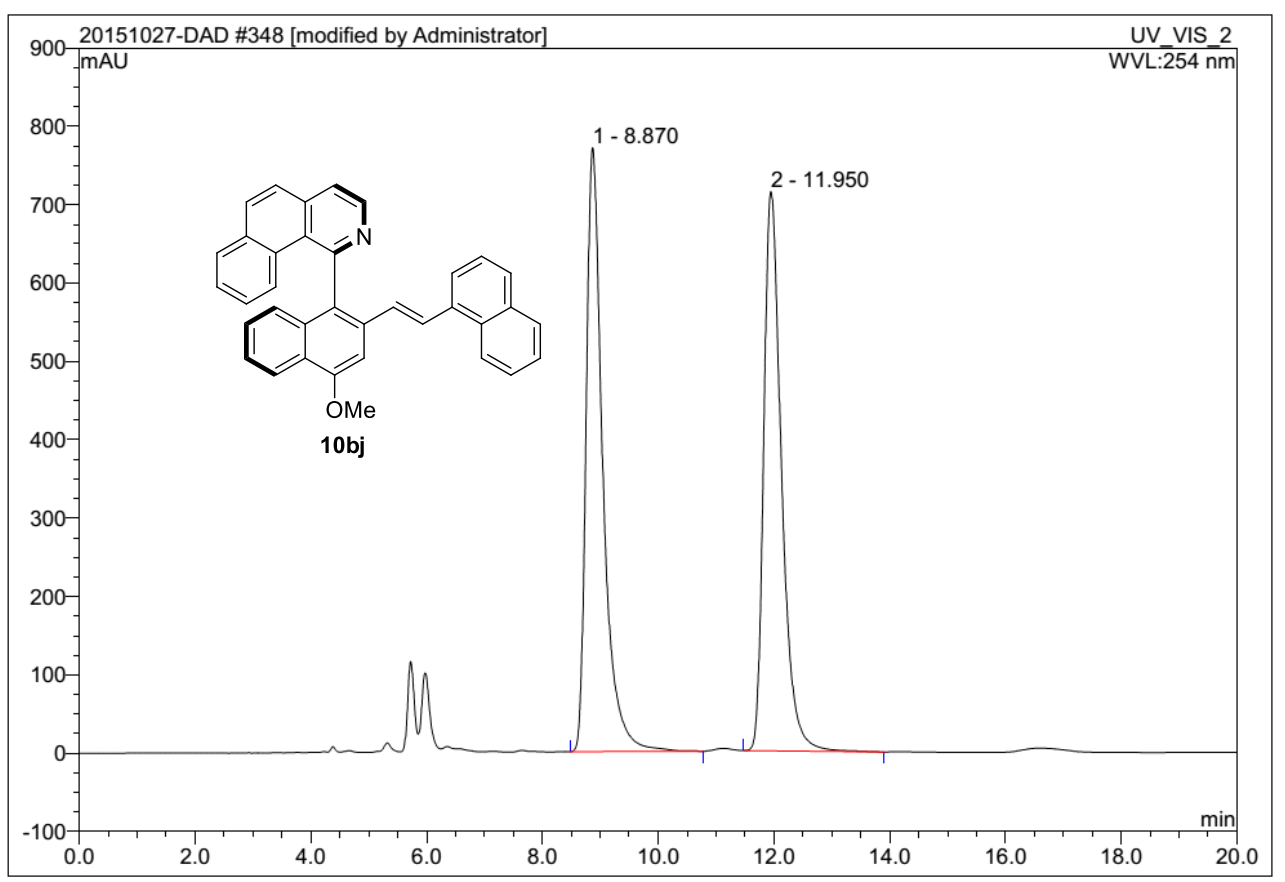

\begin{tabular}{|r|ccccccc|}
\hline No. & $\begin{array}{c}\text { Ret.Time } \\
\text { min }\end{array}$ & Peak Name & $\begin{array}{c}\text { Height } \\
\text { mAU }\end{array}$ & $\begin{array}{c}\text { Area } \\
\text { mAU*min }\end{array}$ & $\begin{array}{c}\text { Rel.Area } \\
\%\end{array}$ & Amount & Type \\
\hline 1 & 8.87 & n.a. & 771.132 & 257.538 & 50.36 & n.a. & BMB $^{*}$ \\
2 & 11.95 & n.a. & 714.410 & 253.879 & 49.64 & n.a. & BMB $^{*}$ \\
\hline Total: & & & 1485.543 & 511.416 & 100.00 & 0.000 & \\
\hline
\end{tabular}

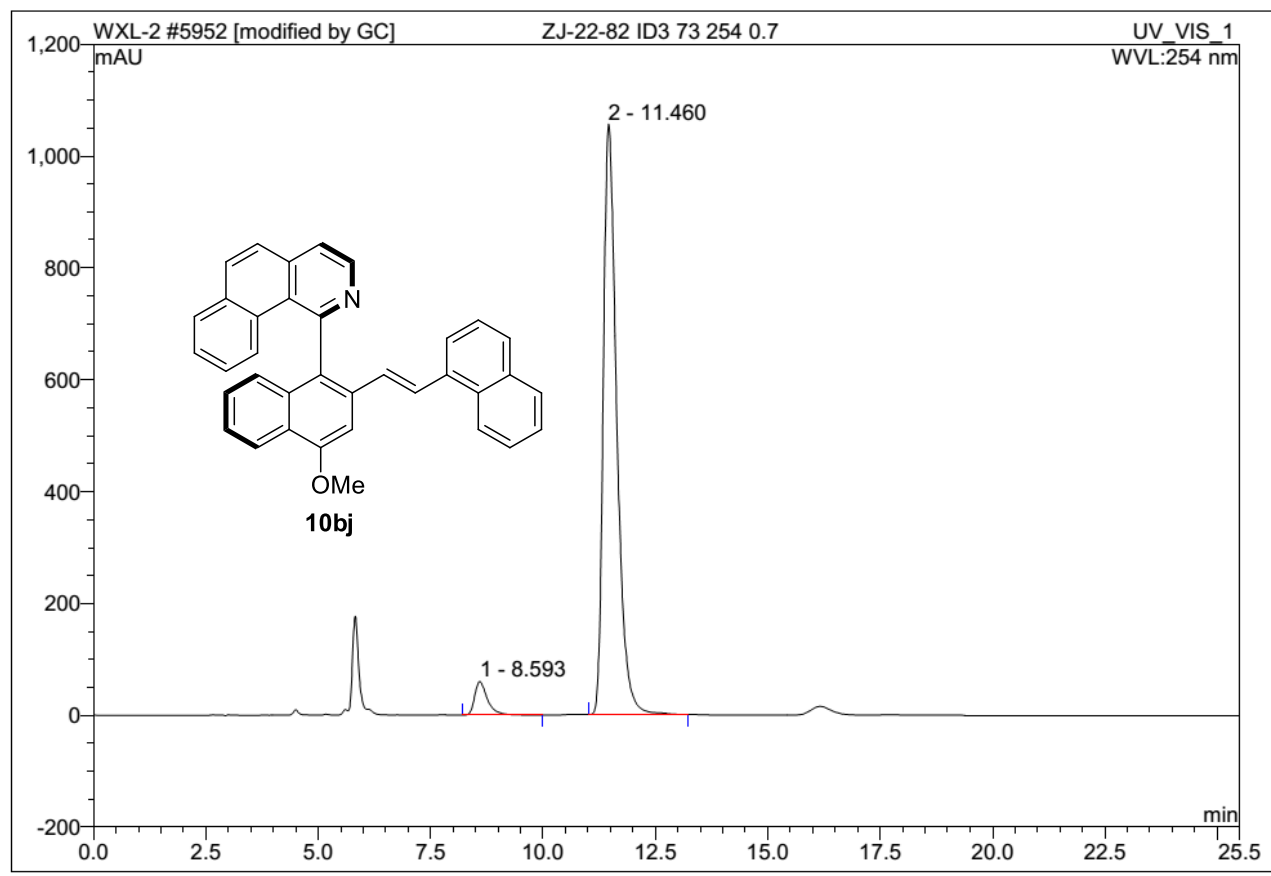

\begin{tabular}{|r|rrrrrrr|}
\hline No. & $\begin{array}{c}\text { Ret.Time } \\
\text { min }\end{array}$ & Peak Name & $\begin{array}{c}\text { Height } \\
\text { mAU }\end{array}$ & $\begin{array}{c}\text { Area } \\
\text { mAU*min }\end{array}$ & $\begin{array}{r}\text { Rel.Area } \\
\%\end{array}$ & Amount & Type \\
\hline 1 & 8.59 & n.a. & 60.074 & 19.809 & 5.13 & n.a. & BMB \\
2 & 11.46 & n.a. & 1056.354 & 366.661 & 94.87 & n.a. & BMB \\
\hline Total: & & & 1116.427 & 386.470 & 100.00 & 0.000 & \\
\hline
\end{tabular}




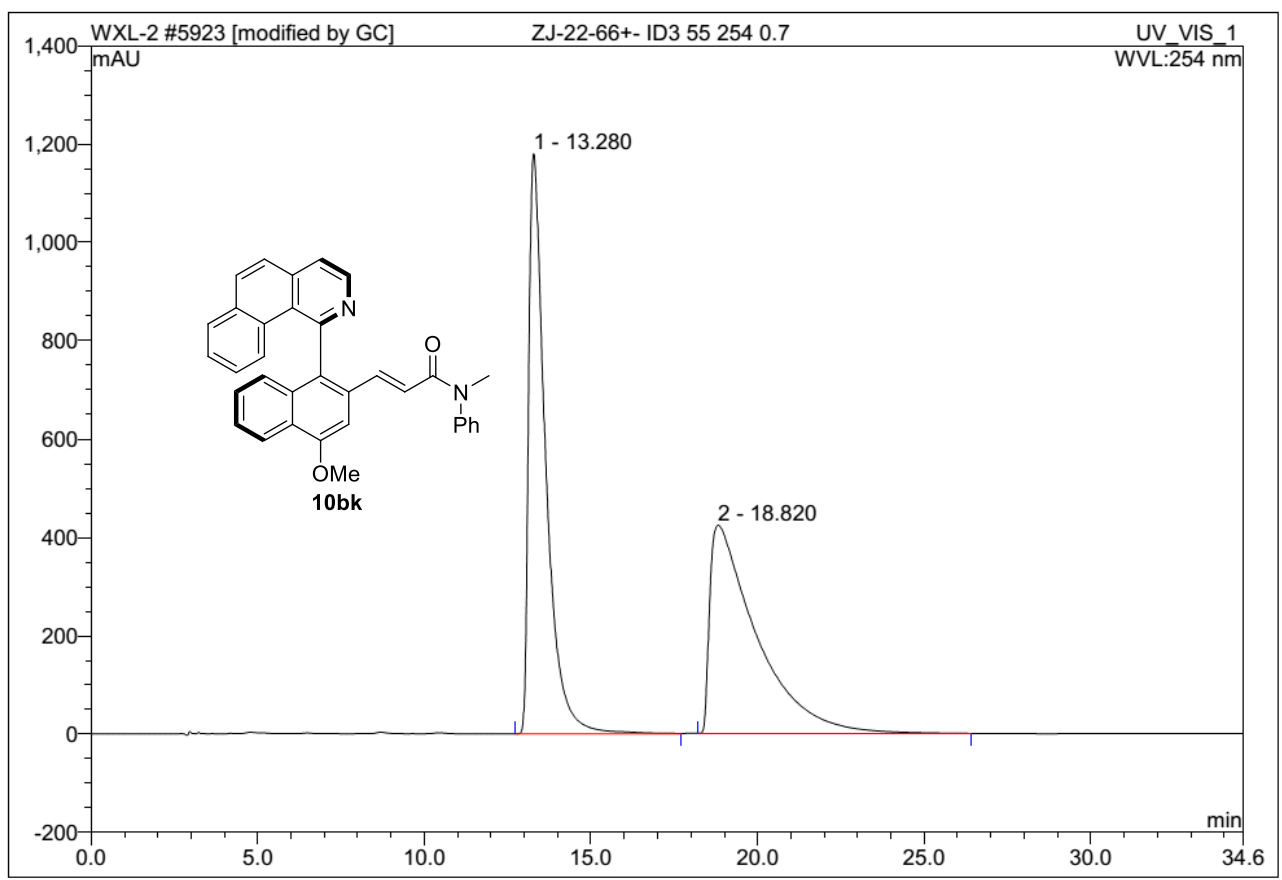

\begin{tabular}{|r|ccrrrrr|}
\hline No. & $\begin{array}{c}\text { Ret.Time } \\
\text { min }\end{array}$ & Peak Name & $\begin{array}{c}\text { Height } \\
\text { mAU }\end{array}$ & $\begin{array}{c}\text { Area } \\
\text { mAU*min }\end{array}$ & $\begin{array}{c}\text { Rel.Area } \\
\%\end{array}$ & Amount & Type \\
\hline 1 & 13.28 & n.a. & 1180.977 & 700.832 & 50.31 & n.a. & BMB \\
2 & 18.82 & n.a. & 424.086 & 692.126 & 49.69 & n.a. & BMB \\
\hline Total: & & & 1605.063 & 1392.958 & 100.00 & 0.000 & \\
\hline
\end{tabular}

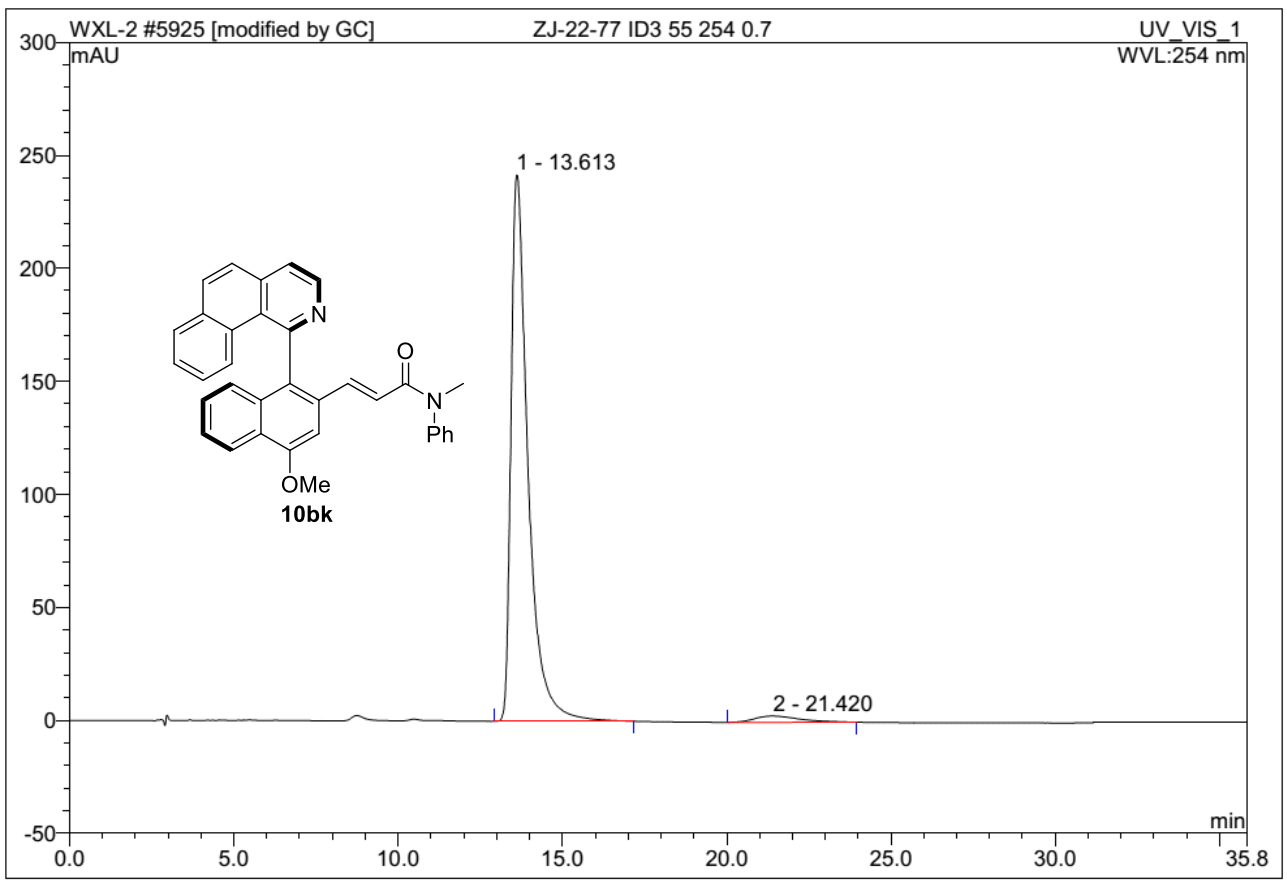

\begin{tabular}{|r|rrrrrrr|}
\hline No. & $\begin{array}{c}\text { Ret.Time } \\
\text { min }\end{array}$ & Peak Name & $\begin{array}{c}\text { Height } \\
\text { mAU }\end{array}$ & $\begin{array}{r}\text { Area } \\
\text { mAU*min }\end{array}$ & $\begin{array}{r}\text { Rel.Area } \\
\%\end{array}$ & Amount & Type \\
\hline 1 & 13.61 & n.a. & 241.860 & 145.150 & 97.06 & n.a. & BMB \\
2 & 21.42 & n.a. & 2.725 & 4.399 & 2.94 & n.a. & BMB \\
\hline Total: & & & 244.585 & 149.548 & 100.00 & 0.000 & \\
\hline
\end{tabular}




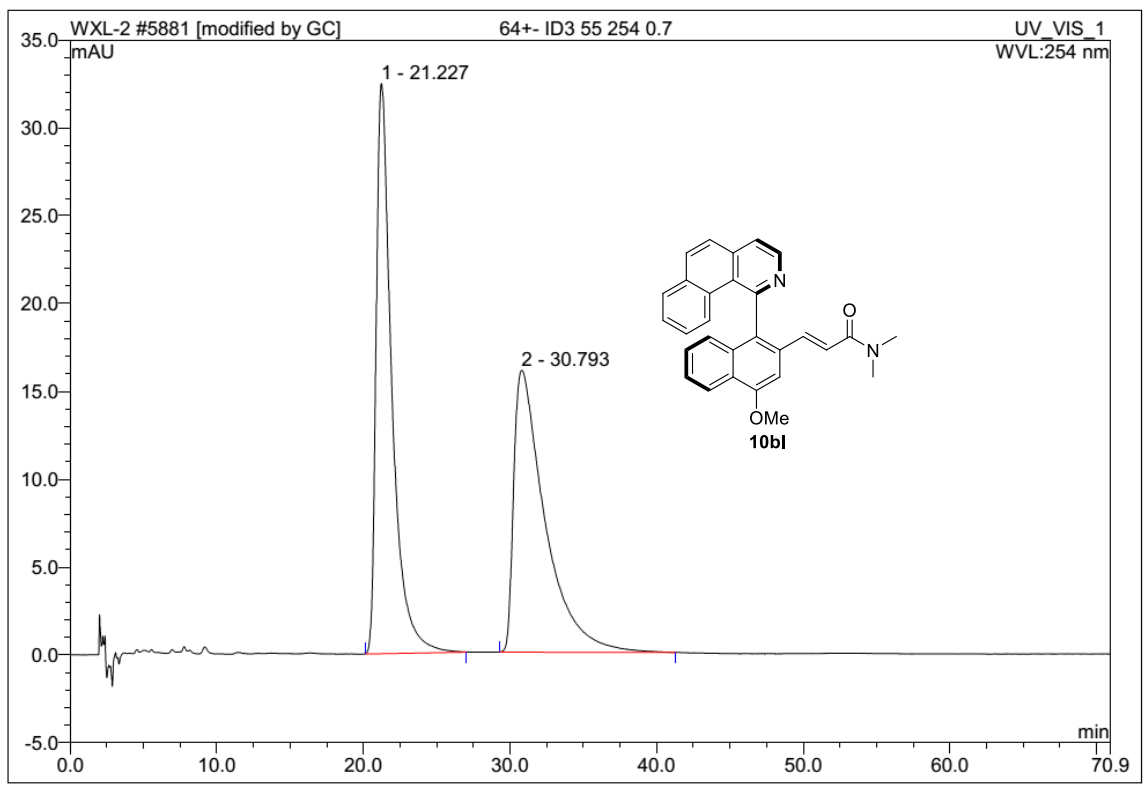

\begin{tabular}{|r|ccccccc|}
\hline No. & $\begin{array}{c}\text { Ret.Time } \\
\text { min }\end{array}$ & Peak Name & $\begin{array}{c}\text { Height } \\
\text { mAU }\end{array}$ & $\begin{array}{c}\text { Area } \\
\text { mAU*min }\end{array}$ & $\begin{array}{c}\text { Rel.Area } \\
\%\end{array}$ & Amount & Type \\
\hline 1 & 21.23 & n.a. & 32.460 & 40.363 & 50.40 & n.a. & BMB $^{*}$ \\
2 & 30.79 & n.a. & 16.046 & 39.724 & 49.60 & n.a. & BMB $^{*}$ \\
\hline Total: & & & 48.506 & 80.087 & 100.00 & 0.000 & \\
\hline
\end{tabular}

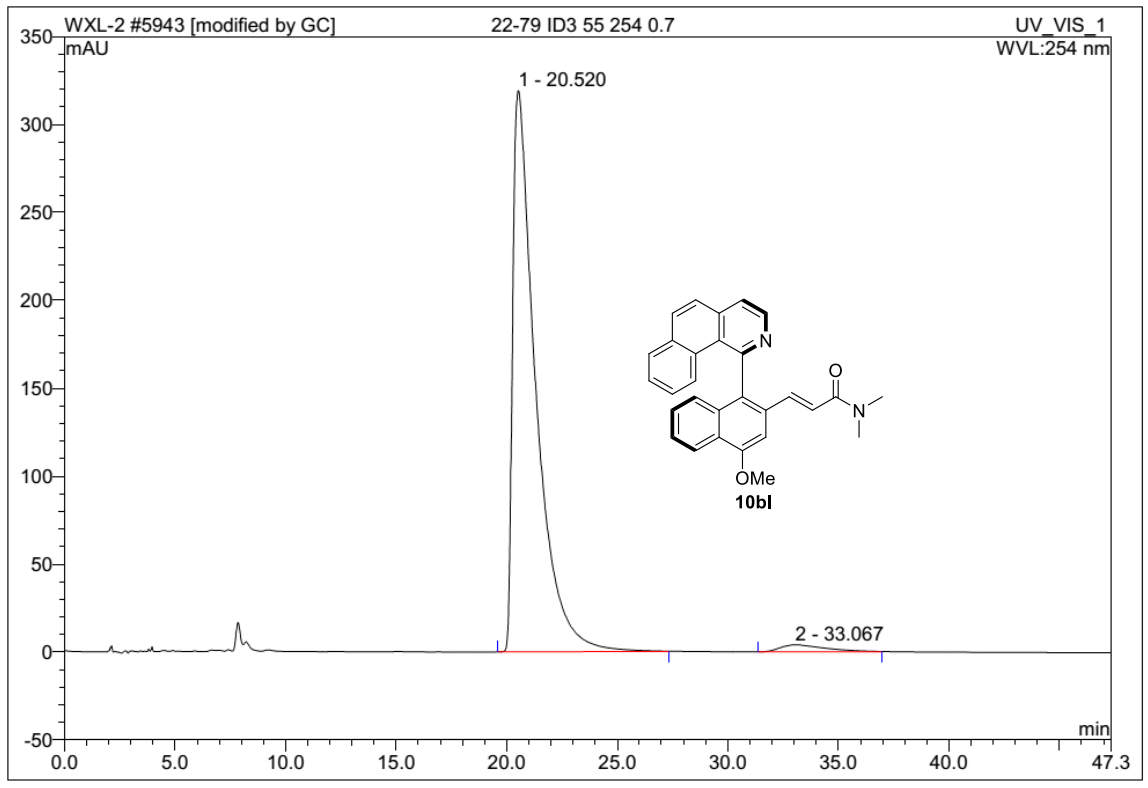

\begin{tabular}{|r|ccrrrrr|}
\hline No. & $\begin{array}{c}\text { Ret.Time } \\
\text { min }\end{array}$ & Peak Name & $\begin{array}{c}\text { Height } \\
\text { mAU }\end{array}$ & $\begin{array}{c}\text { Area } \\
\text { mAUmin }\end{array}$ & $\begin{array}{c}\text { Rel.Area } \\
\%\end{array}$ & Amount & Type \\
\hline 1 & 20.52 & n.a. & 319.368 & 386.850 & 97.63 & n.a. & BMB \\
2 & 33.07 & n.a. & 3.979 & 9.401 & 2.37 & n.a. & BMB \\
\hline Total: & & & 323.347 & 396.252 & 100.00 & 0.000 & \\
\hline
\end{tabular}




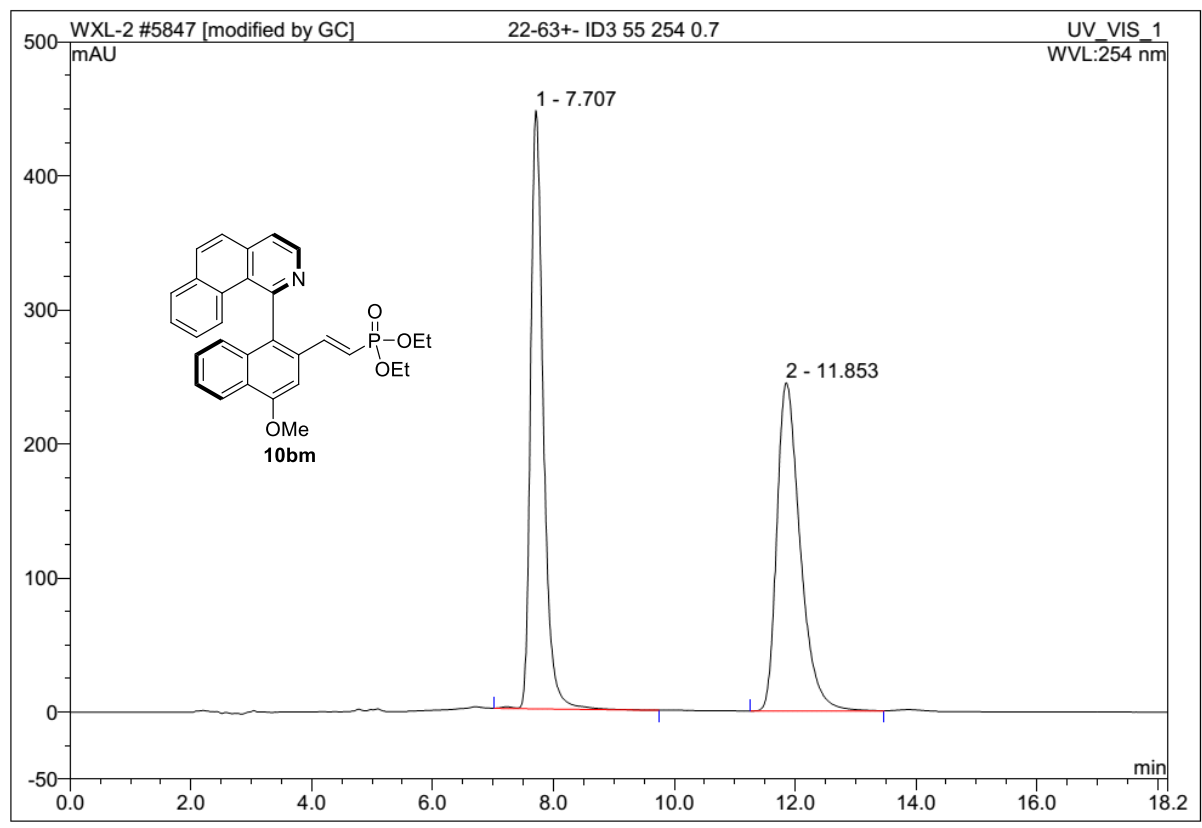

\begin{tabular}{|r|rrrrrrr|}
\hline No. & $\begin{array}{c}\text { Ret.Time } \\
\text { min }\end{array}$ & Peak Name & $\begin{array}{c}\text { Height } \\
\text { mAU }\end{array}$ & $\begin{array}{c}\text { Area } \\
\text { mAU*min }\end{array}$ & $\begin{array}{c}\text { Rel.Area } \\
\%\end{array}$ & Amount & Type \\
\hline 1 & 7.71 & n.a. & 446.531 & 109.203 & 49.95 & n.a. & BMB $^{*}$ \\
2 & 11.85 & n.a. & 245.056 & 109.417 & 50.05 & n.a. & BMB \\
\hline Total: & & & 691.586 & 218.620 & 100.00 & 0.000 & \\
\hline
\end{tabular}

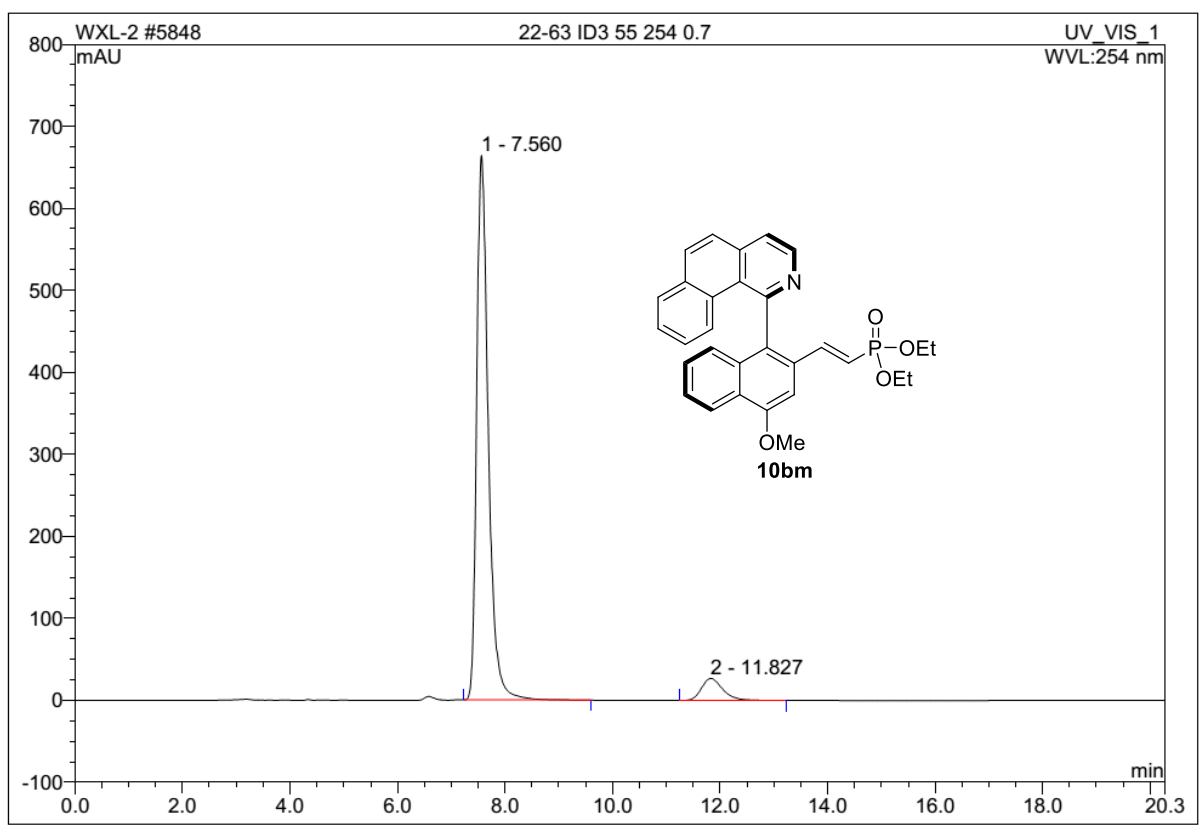

\begin{tabular}{|r|rrrrrrr|}
\hline No. & $\begin{array}{c}\text { Ret.Time } \\
\text { min }\end{array}$ & Peak Name & $\begin{array}{c}\text { Height } \\
\text { mAU }\end{array}$ & $\begin{array}{r}\text { Area } \\
\text { mAU*min }\end{array}$ & $\begin{array}{r}\text { Rel.Area } \\
\%\end{array}$ & Amount & Type \\
\hline 1 & 7.56 & n.a. & 664.647 & 166.707 & 93.13 & n.a. & BMB \\
2 & 11.83 & n.a. & 26.843 & 12.300 & 6.87 & n.a. & BMB \\
\hline Total: & & & 691.489 & 179.006 & 100.00 & 0.000 & \\
\hline
\end{tabular}




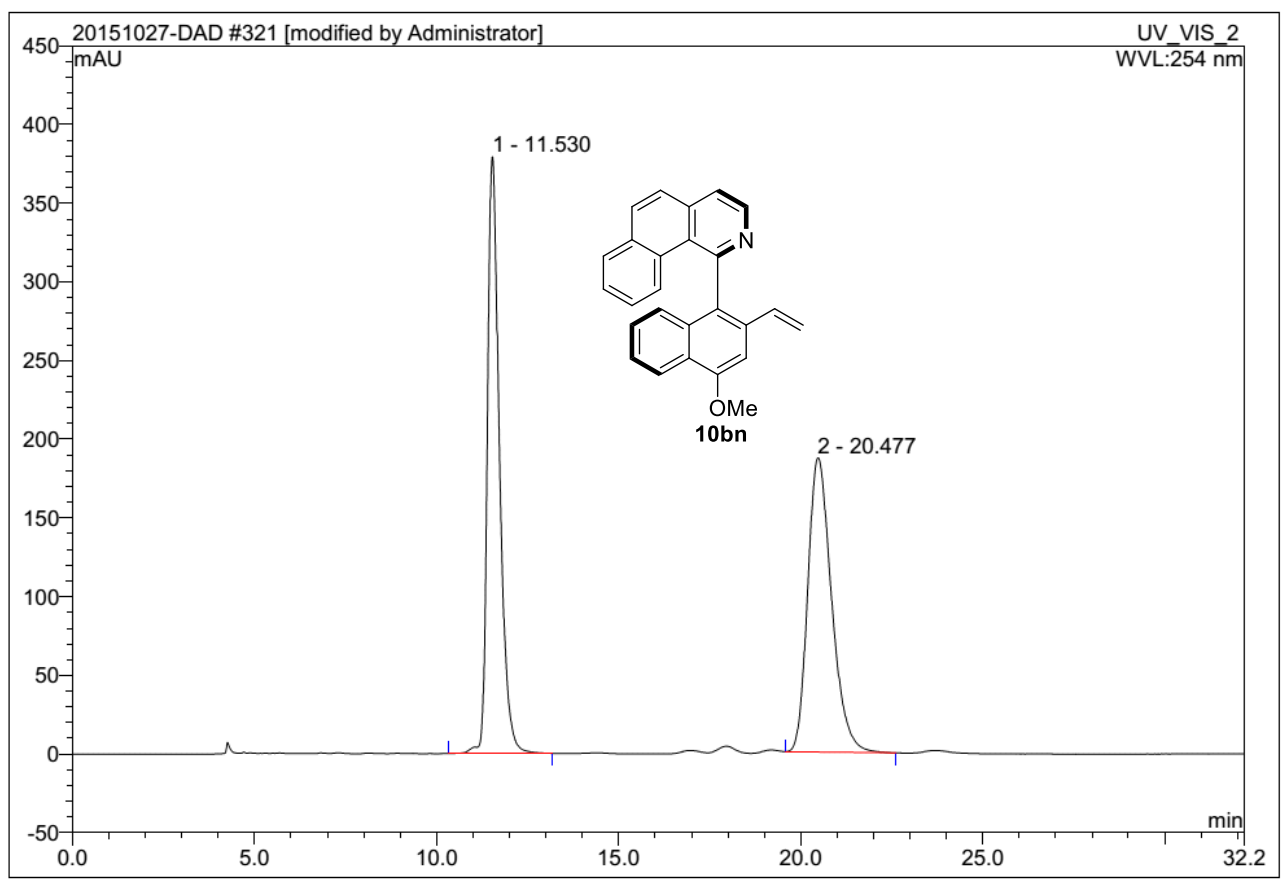

\begin{tabular}{|r|ccccccc|}
\hline No. & $\begin{array}{c}\text { Ret.Time } \\
\text { min }\end{array}$ & Peak Name & $\begin{array}{c}\text { Height } \\
\text { mAU }\end{array}$ & $\begin{array}{c}\text { Area } \\
\text { mAU*min }\end{array}$ & $\begin{array}{c}\text { Rel.Area } \\
\%\end{array}$ & Amount & Type \\
\hline 1 & 11.53 & n.a. & 379.460 & 146.176 & 50.75 & n.a. & BMB \\
2 & 20.48 & n.a. & 186.935 & 141.863 & 49.25 & n.a. & BMB $^{*}$ \\
\hline Total: & & & 566.395 & 288.039 & 100.00 & 0.000 & \\
\hline
\end{tabular}

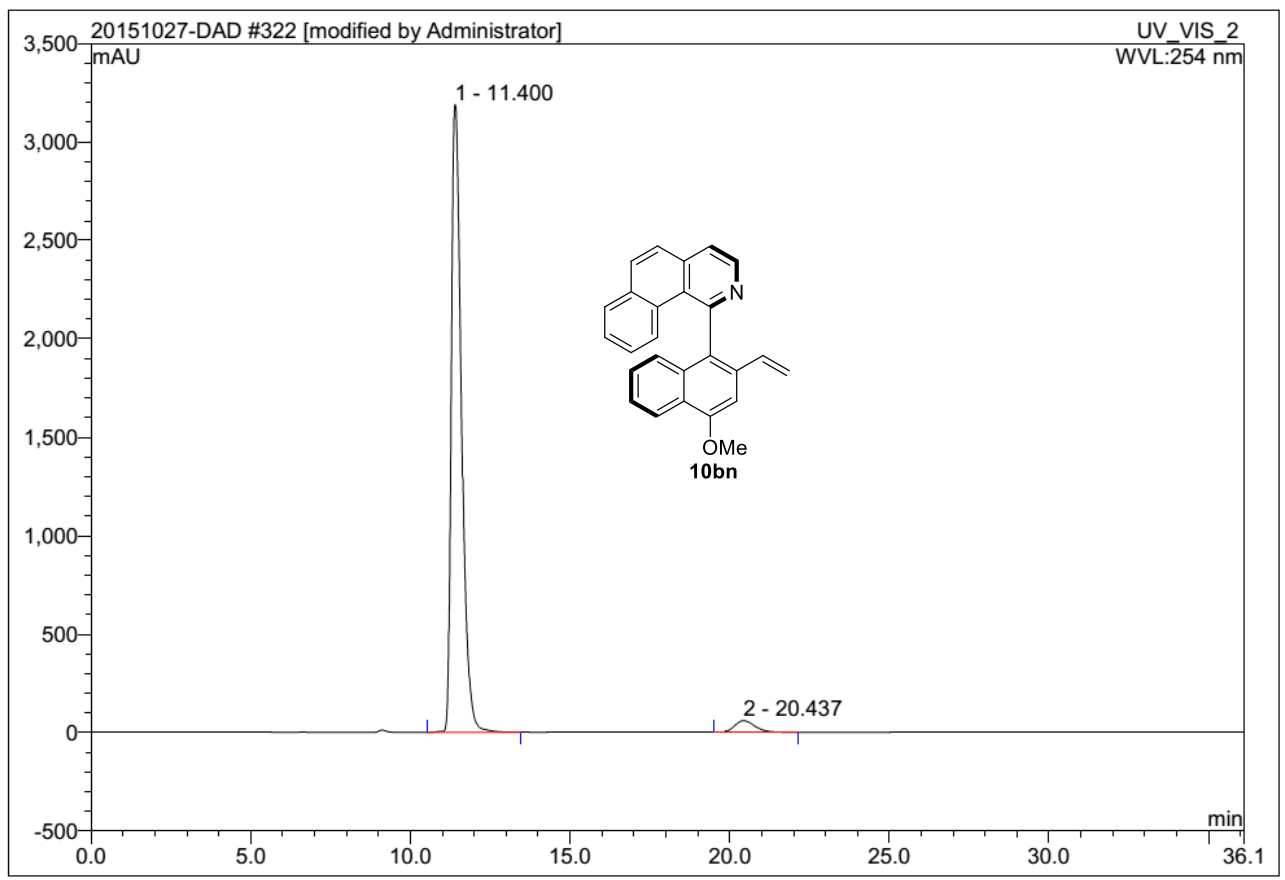

\begin{tabular}{|r|ccrrrrr|}
\hline No. & $\begin{array}{c}\text { Ret.Time } \\
\text { min }\end{array}$ & Peak Name & $\begin{array}{c}\text { Height } \\
\text { mAU }\end{array}$ & $\begin{array}{c}\text { Area } \\
\text { mAU*min }\end{array}$ & $\begin{array}{r}\text { Rel.Area } \\
\%\end{array}$ & Amount & Type \\
\hline 1 & 11.40 & n.a. & 3191.756 & 1187.856 & 96.34 & n.a. & BMB \\
2 & 20.44 & n.a. & 60.263 & 45.189 & 3.66 & n.a. & BMB \\
\hline Total: & & & 3252.019 & 1233.045 & 100.00 & 0.000 & \\
\hline
\end{tabular}

